\title{
Numerical simulation of fine particle separation in hindered -settling bed separators by computational fluid dynamics
}

\author{
Yunkai Xia \\ West Virginia University
}

Follow this and additional works at: https://researchrepository.wvu.edu/etd

\section{Recommended Citation}

Xia, Yunkai, "Numerical simulation of fine particle separation in hindered -settling bed separators by computational fluid dynamics" (2004). Graduate Theses, Dissertations, and Problem Reports. 2614. https://researchrepository.wvu.edu/etd/2614

This Dissertation is protected by copyright and/or related rights. It has been brought to you by the The Research Repository @ WVU with permission from the rights-holder(s). You are free to use this Dissertation in any way that is permitted by the copyright and related rights legislation that applies to your use. For other uses you must obtain permission from the rights-holder(s) directly, unless additional rights are indicated by a Creative Commons license in the record and/ or on the work itself. This Dissertation has been accepted for inclusion in WVU Graduate Theses, Dissertations, and Problem Reports collection by an authorized administrator of The Research Repository @ WVU. For more information, please contact researchrepository@mail.wvu.edu. 


\title{
NUMERICAL SIMULATION OF FINE PARTICLE SEPARATION IN HINDERED-SETTLING BED SEPARATORS BY COMPUTATIONAL FLUID DYNAMICS
}

\author{
Yunkai Xia \\ Dissertation submitted to the \\ College of Engineering and Mineral Resources \\ at West Virginia University \\ in partial fulfillment of the requirements \\ for the degree of \\ Doctor of Philosophy \\ in \\ Mining Engineering \\ Felicia F. Peng, Ph. D., Chair \\ Keith A. Heasley, Ph.D. \\ Bruce Kang, Ph. D. \\ Yi Luo, Ph. D. \\ John W. Zondlo, Ph. D. \\ Mining Engineering Department \\ Morgantown, West Virginia \\ 2004
}

Keywords: Hindered-settling bed separator, CFD, Fine coal, Density separation, Multiple phase flow 


\title{
ABSTRACT \\ NUMERICAL SIMULATION OF FINE PARTICLE SEPARATION IN HINDERED-SETTLING BED SEPARATORS BY COMPUTATIONAL FLUID DYNAMICS
}

\author{
Yunkai Xia
}

The objective of the present study was to improve the understanding of multiple phase flows in hindered-settling bed separators (HSBS). A better understanding of mineral separation in HSBS and the role of structured plates was gained through studies conducted with Computational Fluid Dynamics (CFD) analysis tools. An Euler-Lagrange model from CFD technique is used for this purpose.

In an Euler-Lagrange model, two dimensional incompressible Navier-Stokes equations are solved with the implementation of a finite volume approach over staggered grids with application of Baldwin-Lomax turbulent model. The overall accuracy of the method is second-order in both space and time. The calculation of the liquid field provides the liquid velocity profile in the separator. The integration of movement equations of the particles makes it possible to track the trajectories of discrete particles in the fluid field. The integration of individual particle behavior results in a description of macroscopic behaviors of particle assembly in the fluidized-bed and makes a prediction of density separation using statistical analysis of a number of representative particles. The operating parameters including suspension density set point value, fluidizing water velocity, feed pipe water velocity, feed solid concentration, particle sizes and column geometry were investigated. The simulation has been validated against in-plant test results. Comparisons between the simulations and experiments show the capability of this multiple phase model.

An Euler-Lagrange model has also been developed which simulates the role of structured plates in the HSBS. This device utilizes corrugated plates to improve the performance of conventional hindered-settling bed separators. An investigation utilizing the model was carried out and has predicted an improved separation performance denoted by lower probable errors, lowered processing size limits, and higher throughputs at acceptable separation efficiencies. The model has also predicted that the unfavorable impact of the feed rate fluctuations is reduced significantly by the innovative addition of structured plate design. Experimental results and animation from simulation have verified that the fluid rotation exists between the structured plates to enhance the density separation. Laboratory test results indicate that improvements in separation efficiency can be achieved using the addition of structured plates. The simulation also revealed that the baffled column with structured plates can hold a broader range of suspension densities in response to the fluctuation of solid feed rate than the open column. Finally, the pulsation flows in the presence of the structured plates are simulated. It was found there exists an optimal range for frequency and amplitude of pulsation to improve separation efficiency. 


\section{ACKNOWLEDGEMENTS}

Completion of this dissertation would have been impossible without the guidance and help from my advisors, colleagues, and friends and family.

I am indeed indebted to a number of individuals for their support and advice during this period. Foremost, I would like to thank Dr. Felicia F. Peng, my advisor, for her support, encouragement and advice during my stay in WVU. Her experiences in mineral processing engineering and diligence have constantly inspired me.

I am grateful to Dr. Keith A. Heasley for his guidance and insights into modeling and in organization of the dissertation. I am also grateful to Dr. John W. Zondlo for his guidance in the world of fluid mechanics. Special thanks are also due to Dr. Bruce Kang and Dr. Yi Luo for providing important perspectives to my research work in numerical simulations.

This work was performed under a project sponsored by West Virginia Coal Research Bureau. Their financial support is gratefully acknowledged.

I thank Mr. Zhengxing Gu, graduate student in Mining Engineering, for his friendship and his assistance in building the experimental system and the in-plant testing.

I am especially grateful to my mother and my parents in law in my homeland China for their constant love, encouragements and keeping my family together. Special thanks are due to my sisters for taking over my responsibilities for taking care of my mother.

More importantly, I thank my wife, Ren and our son, Suyu. Their constant love and support have made my life in Morgantown happy and fulfilling. 


\section{TABLE OF CONTENTS}

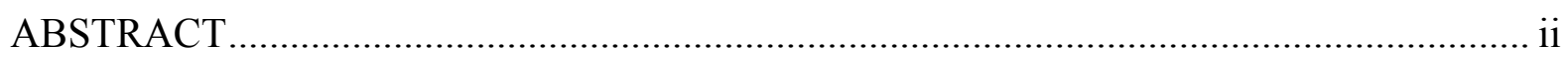

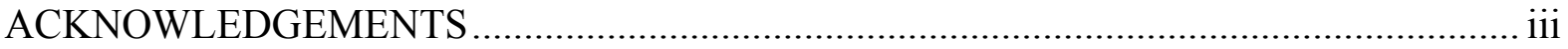

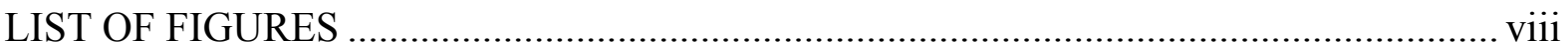

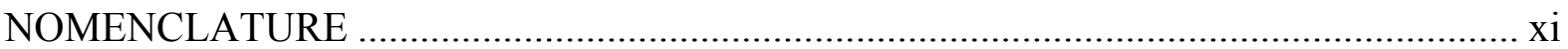

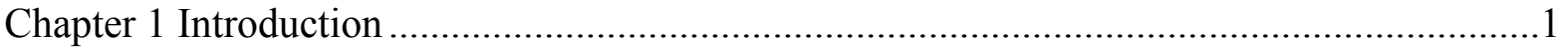

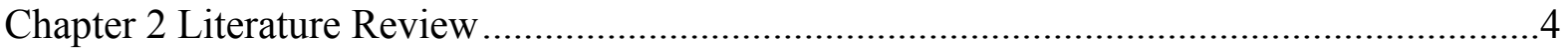

2.1 Hinder-Settling Bed Separator (HSBS) ………….................................................

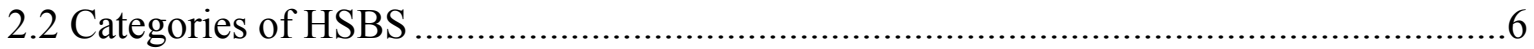

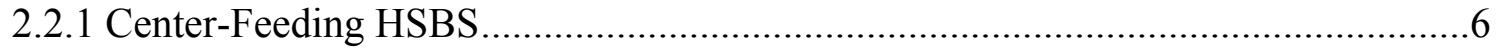

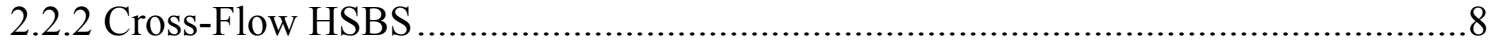

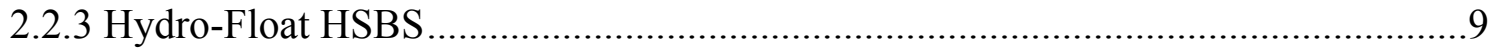

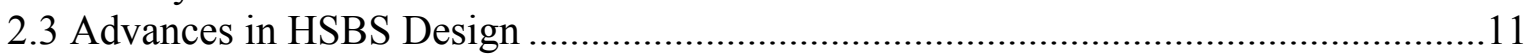

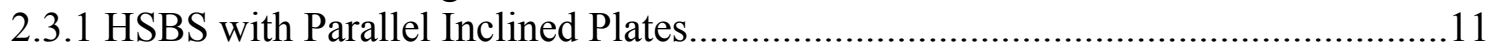

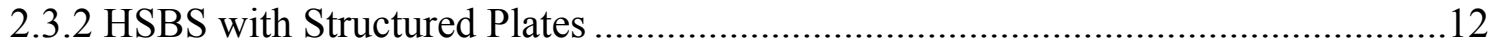

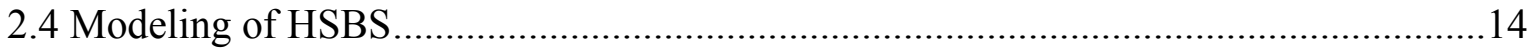

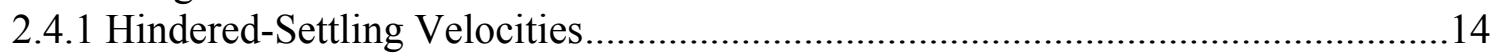

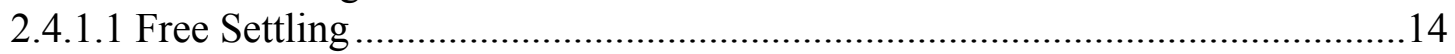

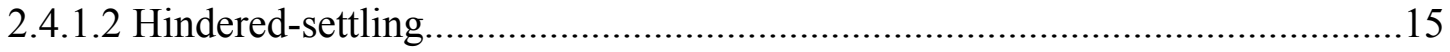

2.4.2 Existing HSBS Models ..............................................................................18

2.4.2.1 Dynamic Population Balance Model ............................................................. 18

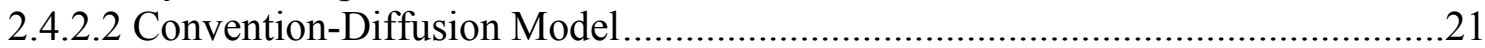

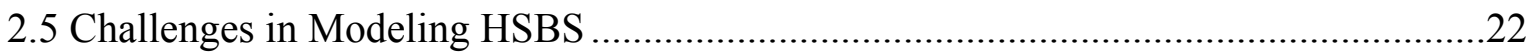

2.6 Application of Multiphase Modeling in Mineral Processing........................................23

2.6.1 Modeling of Jigging ..........................................................................................23

2.6.2 Application of CFD in Multiphase Modeling....................................................26

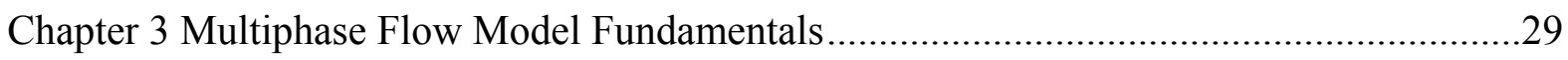

3.1 Basic Physical Models for Liquid Phase ………………........................................29

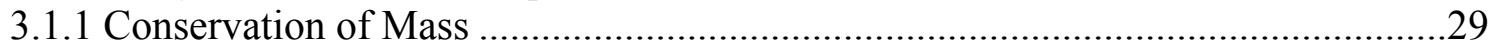

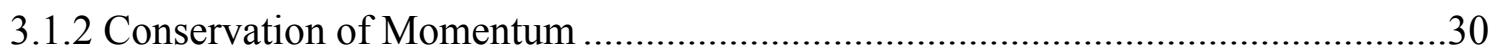

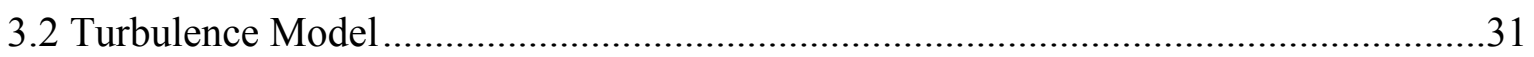

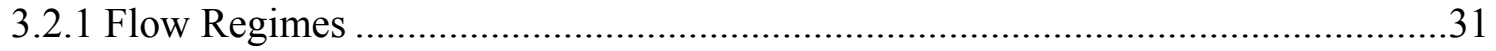

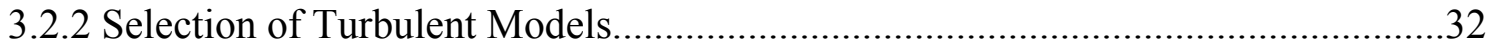

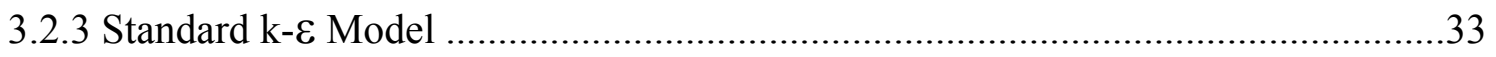

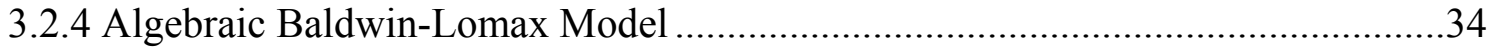

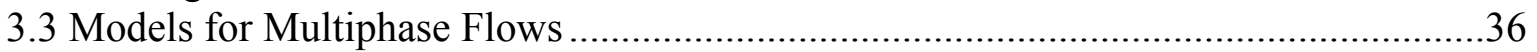

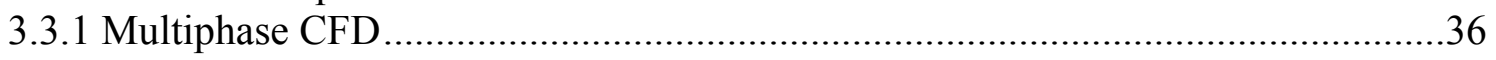


3.3.1.1 Euler-Euler Approach ....................................................................................36

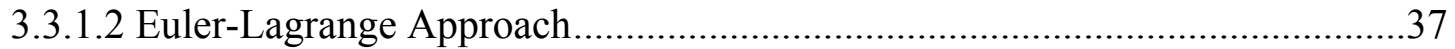

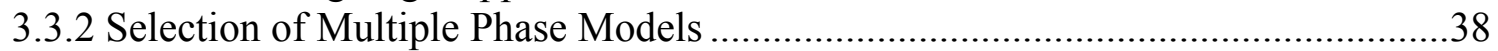

3.4 Fundamentals of Euler-Lagrange Approach.................................................................39

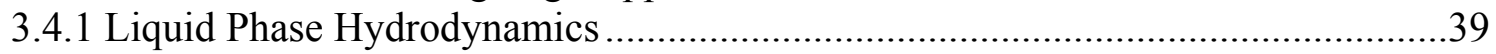

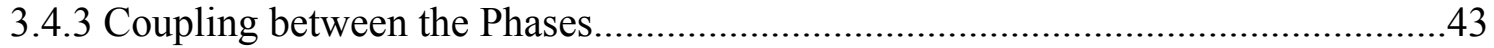

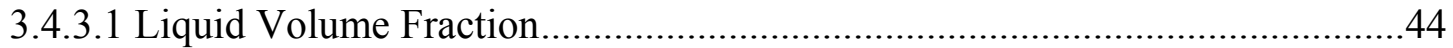

3.4.3.2 Momentum Transfer Term........................................................................4

Chapter 4 Particle Movement and Particle Collisions ………..................................................

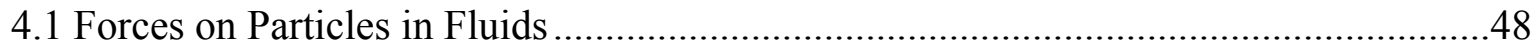

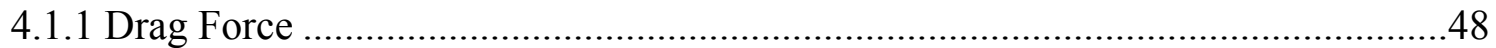

4.1.2 Pressure Gradient and Buoyancy Force..............................................................51

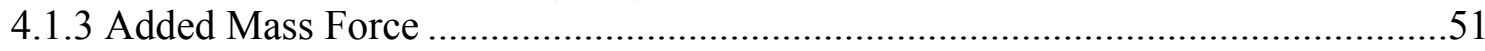

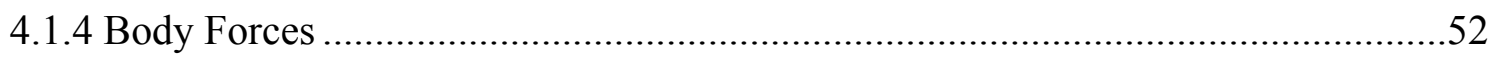

4.1.5 Slip-Shear Lift Force (Saffman Force) ……………..........................................52

4.1.6 Slip-Rotation Lift Force (Mangus Force) ……….............................................54

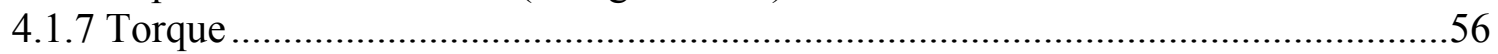

4.2 Particle-Wall and Particle-Particle Collisions ………….............................................57

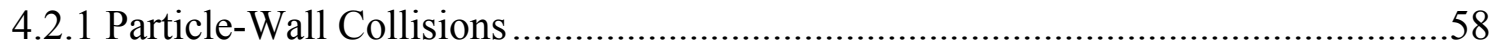

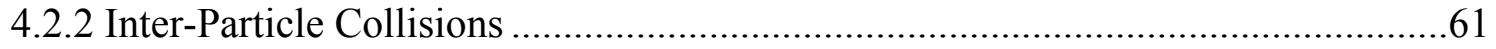

Chapter 5 Boundary Conditions and Numerical Method ..................................................65

5.1 Boundary Conditions and Space Grid Size Control ……………………………........65

5.2 Calculation of Liquid Phase by SIMPLE Approach.....................................................67

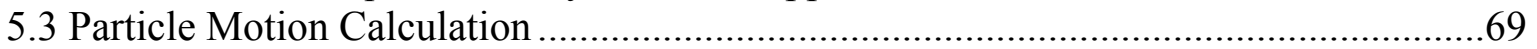

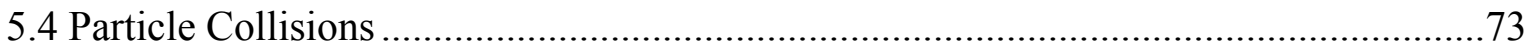

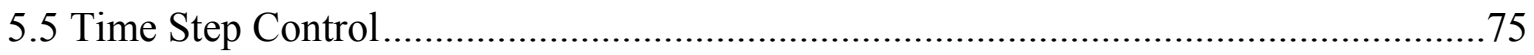

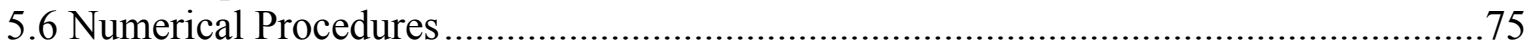

Chapter 6 Hindered-Settling Behavior of Particles ................................................................78

6.1 Behavior of the Isolated Particle........................................................................78

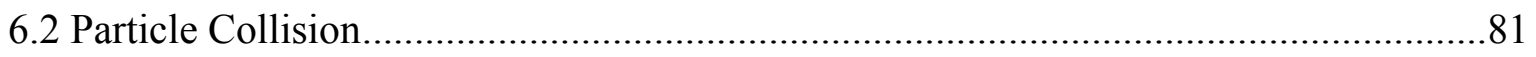

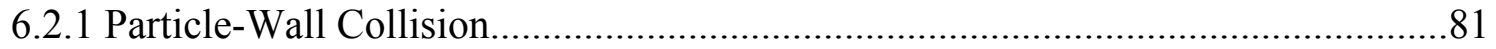

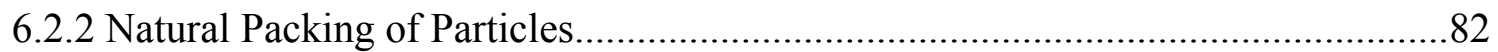

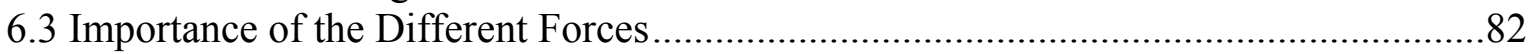

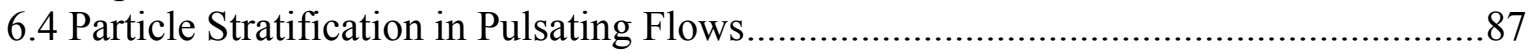

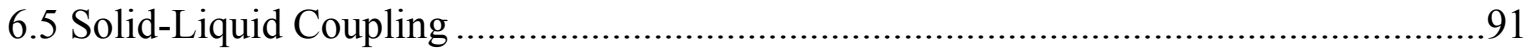

6.6 Effect of Particle Properties on Particle Separation.....................................................94

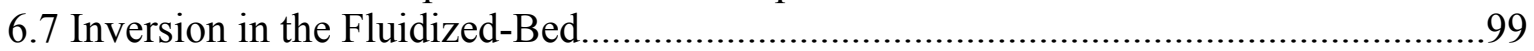

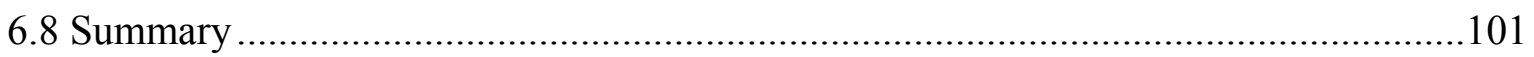

Chapter 7 Particle Density Separation in Hindered-settling Bed Separator ...........................102

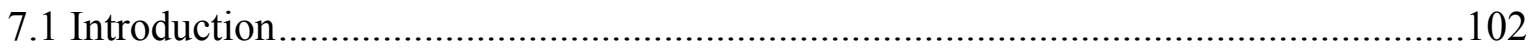


7.1.1 Hydrodynamic Model Basis ............................................................................102

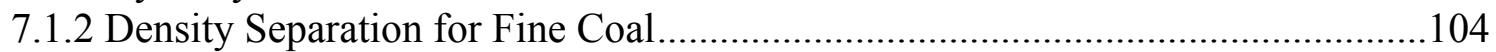

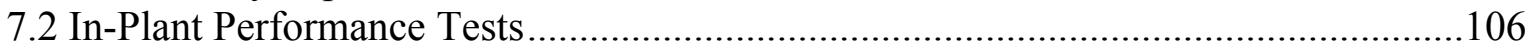

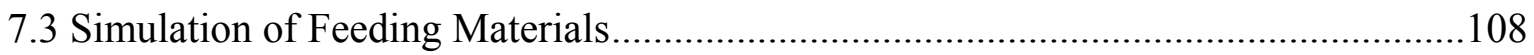

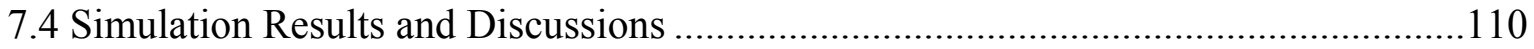

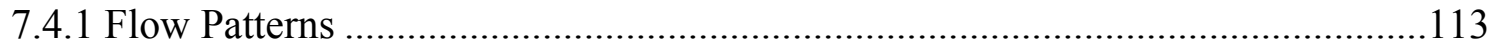

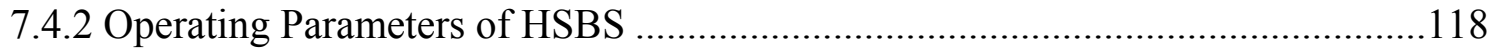

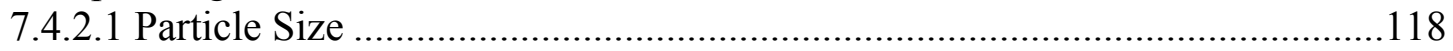

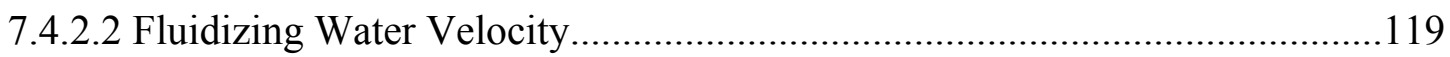

7.4.2.3 Density Set Point.......................................................................................120

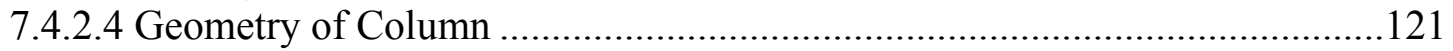

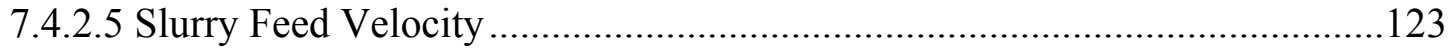

7.4.2.6 Solid Concentration of Feed ....................................................................125

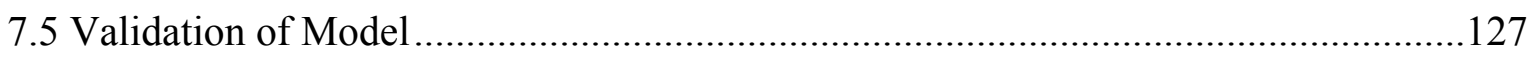

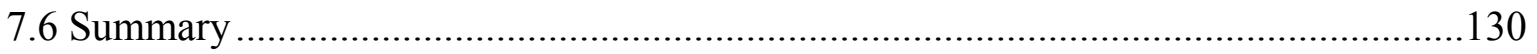

Chapter 8 Influence of Structured Plates in a Liquid Fluidized Bed System ........................131

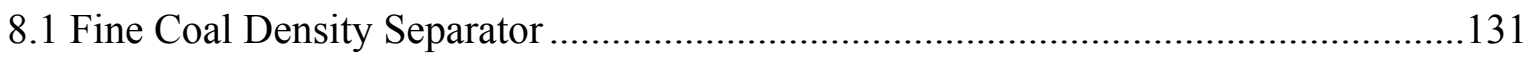

8.2 Laboratory Performance Tests...............................................................................133

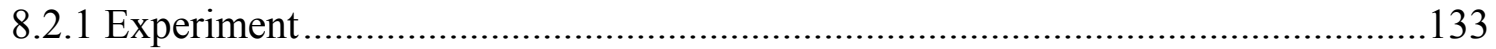

8.2.2 Experiment Results ............................................................................135

8.3 Results and Discussions for Simulation...................................................................136

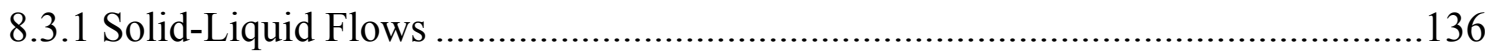

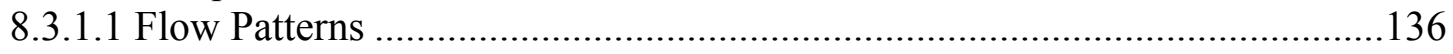

8.3.1.2 Particle Separation ..............................................................................141

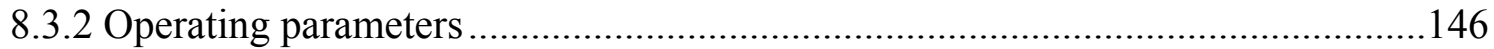

8.3.2.1 Fluidizing Water Velocity.........................................................................146

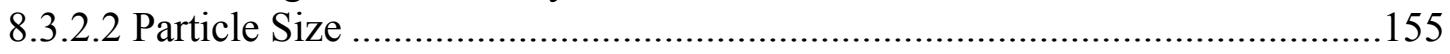

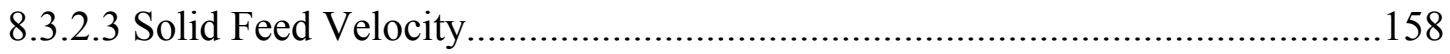

8.3.3 Geometric Parameters of Structured Plates ......................................................162

8.3.3.1 Distance between Structured Plates .............................................................162

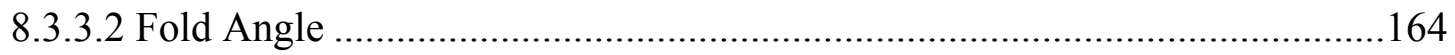

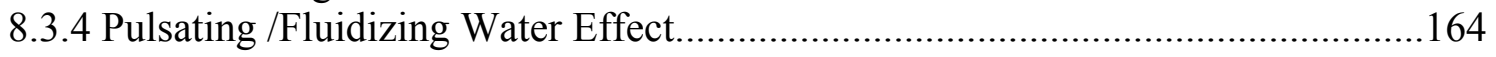

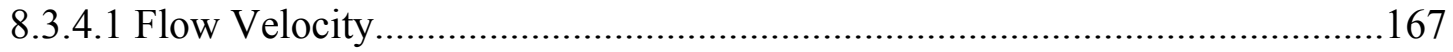

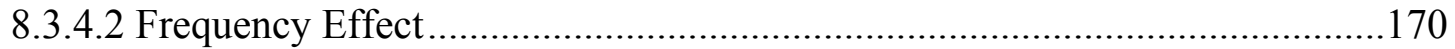

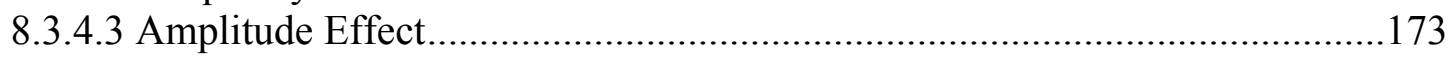

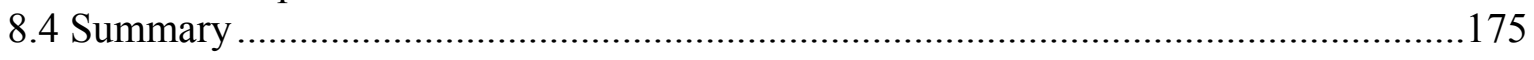

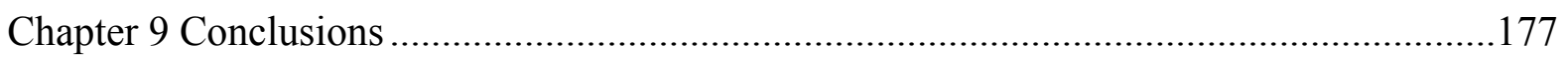

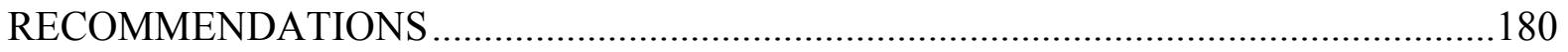

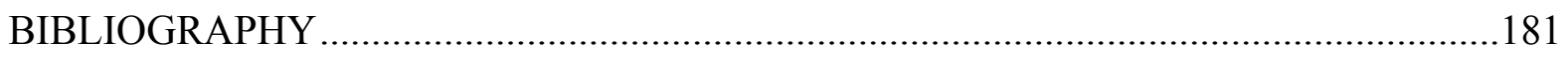

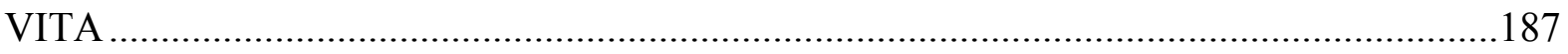




\section{LIST OF TABLES}

Table 3.1 Forces acting on a particle settling in an unsteady and non-uniform liquid ...........42

Table 7.1 Size distribution of fine coal sample (Pittsburgh \#8 coal, OH) ......................... 107

Table 7.2 Washability analysis of fine coal sample....................................................... 107

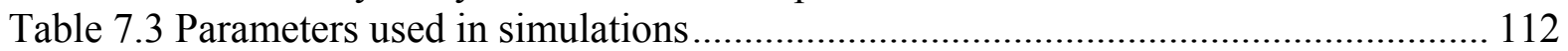

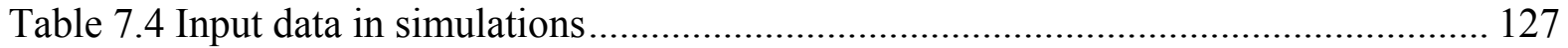

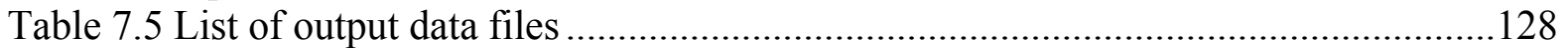

Table 8.1 Density separation performance comparison between open column and baffled

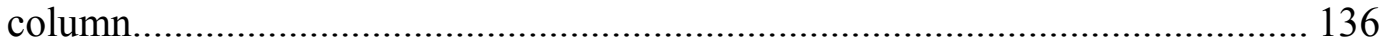

Table 8.2 Parameters used in simulation of the fluidizing water velocity effect at low

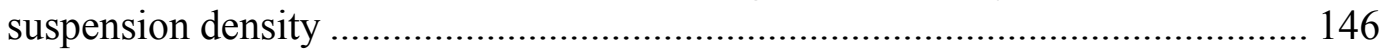

Table 8.3 SG50 and Ep for different columns at different inlet water flow velocity .......... 147

Table 8.4 Parameters used in simulation of fluidizing water velocity effect at high........... 150

Table 8.5 SG50 and Ep for an open column at different inlet water flow velocities at high suspension density

Table 8.6 SG50 and Ep for a baffled column at different inlet water flow velocities at high suspension density

Table 8.7 Parameters used in simulation of particle size effect.

Table 8.8 SG50 and Ep for different size fractions in the open and baffled columns

Table 8.9 Parameters used in simulation of solid feed rate effect at high suspension density

Table 8.10 Variations of SG50 and Ep with solid feed rate in open and baffled columns... 161

Table 8.11 Parameters used in the simulation of fluidizing water velocity effect at low suspension density in the baffled column ...................................................... 170

Table 8.12 SG50 and Ep values for baffled column at various feed water velocities .......... 170

Table 8.13 SG50 and Ep for different columns at different inlet water flow velocities....... 171

Table 8.14 SG50 and Ep values in baffled column at various pulsating frequencies.......... 171

Table 8.15 Parameters used in simulation of pulsating amplitude effect ......................... 173

Table 8.16 SG50 and Ep values for baffled column at various pulsation amplitudes ........ 173 


\section{LIST OF FIGURES}

Figure 2.1 Schematic diagram of advanced fine coal cleaning circuit with HSBS ................ 5

Figure 2.2 Schematic diagrams of center-feeding HSBS............................................ 7

Figure 2.3 Schematic diagram of cross-flow HSBS ….................................................. 9

Figure 2.4 Schematic representation of the Reflux classifier ........................................... 12

Figure 2.5 HSBS with structured plates...................................................................... 13

Figure 2.6 Schematic depicting the primary divisions and flows for the cross-flow

HSBS using the population balance model (Kohmuench, 2000). .....................20

Figure 3.1 Calculation of liquid volume fraction in a computational cell .......................... 44

Figure 3.2 Area-weighted averaging of variables over a computation cell .........................46

Figure 4.1 Drag coefficients as a function of particle Reynolds number for the different

regimes (Source: Schlichting, 1965) .......................................................... 50

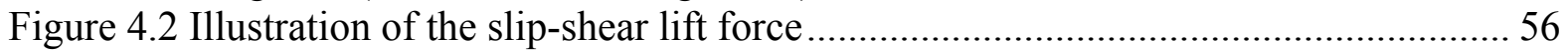

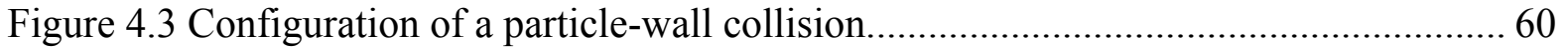

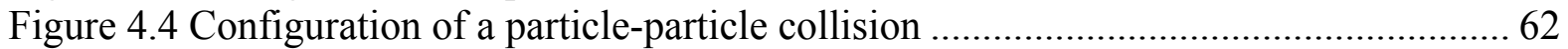

Figure 4.5 Particle-particle collision configurations and co-ordinate system.......................62

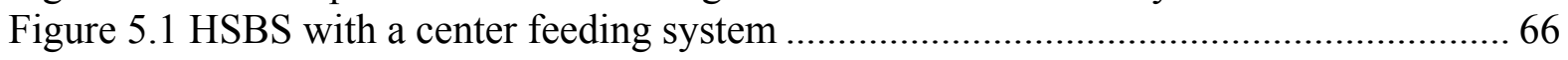

Figure 5.2 Control volume cell .................................................................................. 68

Figure 5.3 Staggered locations arrangement of velocity and scalar values over a grid of

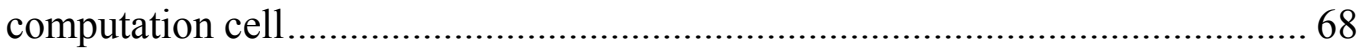

Figure 5.4 Flowchart of application of SIMPLE method ............................................. 70

Figure 5.5 Collision of two particles........................................................................... 74

Figure 5.6 Algorithms for the processing of a sequence of collisions within a constant time

Figure 5.7 Flowchart of principle computational procedures ............................................77

Figure 6.1 Free settling of particles with different sizes and densities .............................. 79

Figure 6.2 Free settling of particles with different sizes and densities ............................... 79

Figure 6.3 Settling of particles with different sizes and densities ................................... 80

Figure 6.4 Hindered-settling of particles with different sizes and densities...................... 80

Figure 6.5 Simulation of trajectory wall-particle collision .......................................... 83

Figure 6.6 The natural packing of particles with different sizes...................................... 84

Figure 6.7 Importance of the different forces with densities for uniform sized particles...... 85

Figure 6.8 Importance of the different forces with sizes for the particles with constant

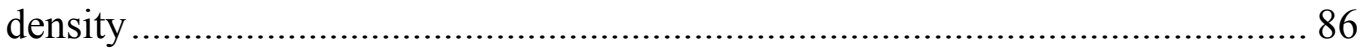

Figure 6.9 Fluctuating movement of particles in a vertical pulsating flow ......................... 89

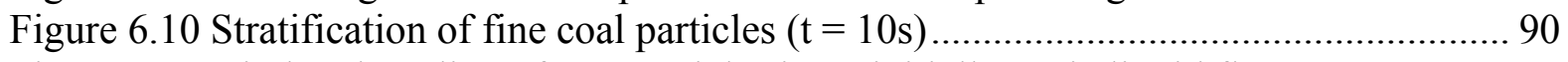

Figure 6.11 Hindered-settling of 400 particles in an initially static liquid flow ................... 92

Figure 6.12 The instant liquid phase velocity vector maps during particle settling ............. 93

Figure 6.13 Hindered-settling of particles with different sizes and a constant density ......... 95

Figure 6.14 Hindered-settling of uniform sized particles with different densities ................ 97

Figure 6.15 Hindered-settling of particles with broad size distribution and density

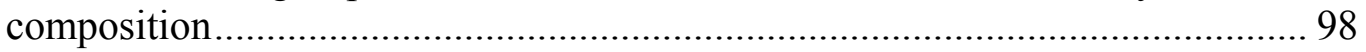

Figure 6.16 Effects of fluidizing water velocity on particle stratification ......................... 100 
Figure 7.1 Schematic diagrams of hindered-settling bed separators with center tangential

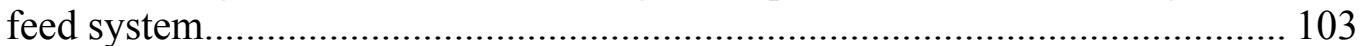

Figure 7.2 Distribution curve for density separation ...................................................... 105

Figure 7.3 Washability of fine coal feed........................................................................ 108

Figure 7.4 Cumulative $w t \%$ as a function of particle size for fine coal feed..................... 110

Figure 7.5 Cumulative $w t \%$ as a function of relative density for fine coal feed ................ 110

Figure 7.6 Liquid velocity field in hindered-settling bed separator with center-tangential feeding system 115

Figure 7.7 Normalized axial velocity for liquid phase at different heights ...................... 115

Figure 7.8 Radial velocity for liquid phase at different heights ..................................... 115

Figure 7.9 Particle Reynolds number distribution in hindered-settling bed separator ........ 116

Figure 7.10 Simulated particle distribution in hindered-settling bed separator.................. 117

Figure 7.11 Effect of particle size on separation performance ....................................... 119

Figure 7.12 Effect of fluidizing water velocities on separation performance...................... 120

Figure 7.13 Effect of suspension density set-point on separation performance ................. 122

Figure 7.14 Effect of different width/height ratio on separation performance ................... 122

Figure 7.15 Effect of slurry feed rate on normalized axial velocity ................................ 124

Figure 7.16 Effect of slurry feed rate on separation performance ................................... 124

Figure 7.17 Effect of solid concentration of feed on normalized axial velocity.................. 126

Figure 7.18 Effect of feed solid concentration on separation performance ........................ 126

Figure 7.19 Distribution curves for size of separation for HSBS ................................... 129

Figure 7.20 Distribution curve for specific gravity of separation for HSBS with a

tangential feed system...............................................................................129

Figure 8.1 Schematic diagram of a HSBS with structured plates .................................... 134

Figure 8.2 Schematic diagrams of open and baffled columns in simulation ...................... 137

Figure 8.3 Axial velocity magnitude map in an open column ......................................... 138

Figure 8.4 Horizontal velocity magnitude map in an open column................................... 138

Figure 8.5 Velocity vector map in an open column ..................................................... 139

Figure 8.6 Axial velocity magnitude map in a baffled column ...................................... 139

Figure 8.7 Horizontal velocity magnitude map in a baffled column ................................ 140

Figure 8.8 Velocity vector map in a baffled column ................................................ 140

Figure 8.9 Axial suspension density peak values at different heights along column .......... 144

Figure 8.10 Particle stratification in a baffled column ................................................... 145

Figure 8.11 Distribution curves at various fluidizing water velocities ............................. 147

Figure 8.12 Distribution curves at various fluidizing water velocities .............................. 148

Figure 8.13 Distribution curves for various fluidizing water velocity in an open column (particle size: $-1.0+0.7 \mathrm{~mm}$ ).................................................................... 152

Figure 8.14 Distribution curves for various fluidizing water velocity in an open column (particle size: $-1.4+1.0 \mathrm{~mm}$ ).............................................................. 152

Figure 8.15 Distribution curves for various fluidizing water velocity in a baffled column

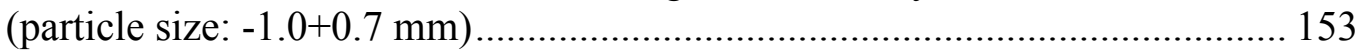

Figure 8.16 Distribution curves for various fluidizing water velocity in a baffled column (particle size: $-1.4+1.0 \mathrm{~mm}$ ) ......................................................................... 153

Figure 8.17 Comparisons of the density cut-point as a function of superficial fluidizing water velocity in an open column and a baffled column 
Figure 8.18 Comparisons of the probable error as a function of superficial fluidizing water velocity in an open column and a baffled column .............................. 154

Figure 8.19 Distribution curves for difference size fractions in an open column................ 156

Figure 8.20 Distribution curves for difference size fractions in a baffled column .............. 156

Figure 8.21 Density cut-point values as a function of particle size for an open column and a baffled column (feed solid concentration: 15\%)

Figure 8.22 Probable error values as a function of particle size for an open column and a baffled column (feed solid concentration: 15\%).

Figure 8.23 Density cut-point values as a function of particle size for an open column and a baffled column (feed solid concentration: $30 \%$ )

Figure 8.24 Probable error values as a function of particle size for an open column and a baffled column (feed solid concentration: 30\%)....

Figure 8.25 Effect of solid feed on separation performance in different columns .............. 163

Figure 8.26 Effect of distance of structured plates in a baffled column ........................... 163

Figure 8.27 Pressure drop along baffled column with distance D ................................... 165

Figure 8.28 Axial water velocity image map with fold angle.......................................... 165

Figure 8.29 Effect of fold angle in a baffled column.................................................. 166

Figure 8.30 Pressure drop along a baffled column with fold angle ................................... 166

Figure 8.31 Wave form of liquid flow ................................................................... 168

Figure 8.32 Effect of fluidizing water velocity on separation performance in a baffled column with pulsating flow .......................................................................... 168

Figure 8.33 Effect of pulsation on SG50 in a baffled column ......................................... 169

Figure 8.34 Effect of pulsation on Ep in a baffled column............................................. 169

Figure 8.35 Effect of pulsation frequency on separation performance in a baffled

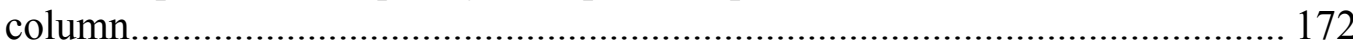

Figure 8.36 Effect of pulsation frequency on SG50 and Ep values in a baffled column...... 172 Figure 8.37 Variations of distribution curves with amplitude in a baffled Column ............ 174 Figure 8.38 Effect of pulsation amplitude on SG50 and Ep values in a baffled column...... 174 


\section{NOMENCLATURE}

\section{Roman Symbols}

\begin{tabular}{|c|c|}
\hline $\mathrm{a}$ & velocity acceleration \\
\hline Ap & cross-section of a spherical particle \\
\hline $\mathrm{Cd}$ & drag coefficient \\
\hline $\mathrm{C}_{\mu}$ & 0.09 , constants in $\mathrm{k}-\varepsilon$ turbulence model \\
\hline $\mathrm{C}_{1 \varepsilon}$ & 1.44 , constants in $\mathrm{k}-\varepsilon$ turbulence model \\
\hline $\mathrm{C}_{2 \varepsilon}$ & 1.92 , constants in $\mathrm{k}-\varepsilon$ turbulence model \\
\hline $\mathrm{C}_{\mathrm{LR}}$ & slip-shear lift coefficient \\
\hline $\mathrm{C}_{\mathrm{LS}}$ & slip-rotation lift coefficient \\
\hline $\mathrm{d}$ & diameter of the particle \\
\hline $\mathrm{D}$ & particle diffusion coefficient \\
\hline $\mathrm{D}_{\mathrm{p}}$ & diameter of a particle \\
\hline DT & time step in Euler-Lagrange method \\
\hline e & $\begin{array}{l}\text { restitution ratio of the normal velocity components after } \\
\text { collision }\end{array}$ \\
\hline Ep & probable error in density separation \\
\hline $\mathrm{F}(\alpha)$ & function of particle and size \\
\hline $\mathrm{F}_{\mathrm{AD}}$ & the added mass force \\
\hline $\mathrm{F}_{\mathrm{B}}$ & body force \\
\hline $\mathrm{F}_{\mathrm{D}}$ & drag force \\
\hline $\mathrm{Fg}$ & gravity force \\
\hline $\mathrm{F}_{\mathrm{LS}}$ & slip-shear lift force \\
\hline $\mathrm{F}_{\mathrm{M}}$ & Mangus force \\
\hline$F_{P}$ & pressure gradient force \\
\hline $\mathrm{F}_{\mathrm{S}}$ & Saffman force \\
\hline $\mathrm{Fi}$ & the component of body force in i direction \\
\hline$F_{\text {total }}$ & all the relevant applicable forces acting on the particle in fluid \\
\hline $\mathrm{F}_{\mathrm{w}}$ & moment transfer terms between solid and liquid phases \\
\hline g & gravitational acceleration \\
\hline $\mathrm{g}_{\mathrm{i}}$ & acceleration in i direction \\
\hline $\mathrm{G}_{\mathrm{k}}$ & production of turbulent kinetic energy \\
\hline Ip & moment of inertia for a particle \\
\hline $\mathrm{Jx}, \mathrm{Jy}, \mathrm{Jz}$ & components of the impulsive forces \\
\hline $\mathrm{k}$ & kinetic energy \\
\hline $\mathrm{K}_{\mathrm{if}}$ & $\begin{array}{l}\text { a fluid counter flow factor which addresses the displacement } \\
\text { of water by settling particles }\end{array}$ \\
\hline $\mathrm{K}_{\mathrm{ic}}$ & $\begin{array}{l}\text { a factor that addresses turbulence caused by cluster of settling } \\
\text { particles }\end{array}$ \\
\hline L & the characteristic length of the duct, $\mathrm{m}$ \\
\hline $\mathrm{m}_{\mathrm{p}}$ & mass of a particle \\
\hline
\end{tabular}




\begin{tabular}{|c|c|}
\hline $\mathrm{n}$ & exponent power index \\
\hline $\mathrm{p}$ & pressure \\
\hline $\mathrm{P}_{\mathrm{d}}$ & $\begin{array}{l}\text { probability for a particle to be generated with a predefined } \\
\text { density }\end{array}$ \\
\hline $\mathrm{P}_{\mathrm{s}}$ & probability for a particle to be generated with a predefined size \\
\hline $\mathrm{R}$ & radius of a particle \\
\hline $\mathrm{R}$ & mass recovery of a particle \\
\hline $\mathrm{R}_{\mathrm{ab}}$ & distance between particle $a$ and $b$ \\
\hline $\operatorname{Re}$ & Reynolds number \\
\hline $\operatorname{Re}_{\mathrm{p}}$ & particle Reynolds number \\
\hline $\mathrm{Re}_{\mathrm{R}}$ & particle rotation Reynolds number \\
\hline $\operatorname{Re}_{\mathrm{S}}$ & Reynolds number of the shear flow \\
\hline SG & specific gravity \\
\hline SG50 & density cut-point in density separation \\
\hline $\mathrm{S}_{\mathrm{m}}$ & source term of mass \\
\hline $\mathrm{t}$ & time \\
\hline $\mathrm{T}$ & torque acting on the particle \\
\hline $\mathrm{T}_{\mathrm{ab}}$ & collision time between particles $\mathrm{a}$ and $\mathrm{b}$ \\
\hline $\mathrm{U}$ & the mean velocity of the flow \\
\hline $\mathrm{U}$ & free settling velocity of isolated particle \\
\hline $\mathrm{U}_{\mathrm{f}}$ & velocity of liquid \\
\hline$u_{i}$ & velocity component in i coordinate \\
\hline $\mathrm{U}_{\mathrm{p}}$ & instant velocity of particle \\
\hline $\mathrm{U}_{\mathrm{Rx}}, \mathrm{U}_{\mathrm{Ry}}, \mathrm{U}_{\mathrm{Rz}}$ & relative velocity in the physical space \\
\hline Us & velocity of a solid particle \\
\hline $\mathrm{U}_{\text {slip }}$ & $\begin{array}{l}\text { slippery velocity of between a solid particle and liquid flow, } \\
\mathrm{m} / \mathrm{s}\end{array}$ \\
\hline $\mathrm{U}_{\mathrm{t}}$ & terminal velocity of a particle \\
\hline $\mathrm{V}_{\mathrm{ab}}$ & relative velocity between particle $a$ and $b$ \\
\hline $\mathrm{V}_{\text {cell }}$ & the volume of a computational cell \\
\hline $\mathrm{V}_{\mathrm{hi}}$ & hindered-settling velocity \\
\hline $\mathrm{V}_{\mathrm{p}}$ & the volume of a particle \\
\hline $\mathrm{V}_{\mathrm{t}}$ & terminal velocity of a particle \\
\hline $\mathrm{V}_{\mathrm{oi}}$ & free settling velocity of a particle \\
\hline $\mathrm{u}, \mathrm{v}, \mathrm{w}$ & Cartesian velocities in $\mathrm{x}, \mathrm{y}$ and $\mathrm{z}$ direction \\
\hline $\mathrm{U}_{\mathrm{p}}, \mathrm{V}_{\mathrm{p}}, \mathrm{W}_{\mathrm{p}}$ & the translational velocity components \\
\hline $\mathrm{W}$ & weight percentage of the particles \\
\hline $\mathrm{X}_{\mathrm{i}}$ & Cartesian coordinates \\
\hline$(\mathrm{x}, \mathrm{y}, \mathrm{z})$ & Cartesian coordinates \\
\hline $\mathrm{X}_{\mathrm{p}}$ & the coordinate of particle \\
\hline
\end{tabular}

Greek Symbols

$\alpha_{\mathrm{f}} \quad$ suspension voidage 
$\beta$

$\delta \mathrm{ij}$

$\delta \mathrm{x}, \delta \mathrm{y}$

$\varepsilon$

$\mu$

$\mu_{\mathrm{d}}$

$\mu_{\mathrm{f}}$

$\mu_{\mathrm{o}}$

$\mu_{\mathrm{t}}$

$v$

$\phi$

$\pi$

$\rho$

$\rho_{\mathrm{p}}$

$\rho_{\mathrm{s}}$

$\rho_{\text {susp }}$

$\sigma_{\mathrm{k}}$

$\sigma_{\varepsilon}$

$\tau_{\mathrm{ij}}$

$\tau_{\mathrm{p}}$

$\omega_{\mathrm{F}}$

$\omega_{\mathrm{p}(\mathrm{x}, \mathrm{y}, \mathrm{z})}$

$\Delta \mathrm{x}, \Delta \mathrm{y}$

$\Delta \mathrm{t}$

$\Phi_{\mathrm{i}, \mathrm{j}}$,

$\Phi_{\mathrm{p}}$

$\Gamma$

unknown function of particle size and shape

the kronecher delta

distance of a particle from the neighbor grid node

rate of dissipation

dynamic viscosity

dynamic coefficient of friction

dynamic viscosity of liquid

static coefficient of friction

turbulent viscosity

kinematic viscosity

solid volume fraction

pi, 3.14159265...

density of water

density of a particle

density of solid

density of suspension

1.0 , constant in $\mathrm{k}-\varepsilon$ turbulence model

1.3 , constant in $\mathrm{k}-\varepsilon$ turbulence model

shear tensor

particle response time

liquid angular velocity

particle angular velocity

grid spacing in the physical space

time step

general variable on the grid nodes

general variable of the particle

diffusion coefficient of liquid

\section{Other Symbols}

$\partial$

partial derivative operator

\section{Superscripts}
$\mathrm{x}, \mathrm{y}, \mathrm{z}$
Cartesian coordinates
$*$
index after collision 


\section{Subscripts}

$\begin{array}{ll}\mathrm{i}, \mathrm{j}, \mathrm{l} & \text { Cartesian coordinates } \\ \mathrm{x}, \mathrm{y}, \mathrm{z} & \text { Cartesian coordinates } \\ \mathrm{f} & \text { fluid } \\ \mathrm{p} & \text { particle } \\ \mathrm{R} & \text { rotation }\end{array}$

Abbreviations

CFD Computational fluid dynamics

DEM Discrete element method

HSBS Hindered-settling bed separator

LES Large eddy simulation

SIMPLE Semi-implicit pressure linked equation 


\section{Chapter 1 Introduction}

As mineral resources mined at lower grades, more finely disseminated and more chemically complex, more efficient separation equipment are needed for fine mineral processing. The research and development of more efficient water-based gravity separation methods meet these needs for fine particles. Gravity separation equipment for fine mineral and coal includes the hinder-settling bed separator (HSBS) and the baffled HSBS with structured plates. HSBS is also known as a liquid-solid fluidized separator.

The reliable design and scale-up of HSBS can not be done without a better understanding of the multiphase (solid-liquid) flow phenomena in this equipment. Also, multiphase flows are of fundamental importance in many reactor or separator concepts in today's chemical, bio-processing and mineral industries etc.

Recently, some advances and innovations have been made in the operation and design of HSBS. This includes implementations of control systems and the development of zigzag structured plates or inclined plates to improve capacity and efficiency.

Some macroscopic type models include the dynamic population balance model and solid concentration convection-diffusion model that have been developed to simulate mineral separation in HSBS. These models are based on an empirical derivation of hindered particle settling velocities in an assumed liquid phase flow. The general applicable separation models which include flow patterns are still unavailable. This can be due to lack of an accurate estimation method for the liquid phase flow. The design of HSBS has so far been carried out 
mainly by means of empirical and semi-empirical correlations, which have been gained from experimental data for separators of different scales. While a strong experimental foundation of such correlations provides security for the applications, transferability to other situations (e. g. different minerals or minerals with a broad range of size and density distributions, or equipment having complex geometry) is usually very limited. Thus, in many cases, trial-anderror schemes or time-consuming scale-up experiments are necessary to achieve satisfactory performance of the large-scale separator system.

For fluid flow modeling, Computational Fluid Dynamics (CFD) is gaining importance in general process applications. CFD has been an important tool in the air and space industry and vehicle design for a long time, where it has to a large extent replaced time-consuming and expensive wind tunnel experiments. However, these applications are primarily singlephase flow, modeling application in mineral separators, in most cases, involves multiphase flows. The modeling and numerical treatment of such flows introduce additional challenges due to numerical problems in solving the resulting equation systems, and a lack in corresponding computational power. Therefore, multiphase CFD applications have gained broad attention only during the last decade when increased computational power available in personal computing systems has enabled computations which were previously considered unfeasible.

The overall objective of this study is to obtain a better understanding of the factors affecting the behavior of hindered-settling bed separators. To reach this goal, the emphasis of this study will focus on:

- Modeling the physical processes of the hindered-settling process in a solid-liquid system using an Euler-Lagrange approach. The turbulent model for liquid phase, 
motion equations for particles, inter-phase coupling effects, particle collision models are specified and tested for batch and continuous tank separation simulation.

- Identifying operating and boundary conditions that will be utilized in this simulation. They are obtained on the basis of both theoretical analysis and real engineering practices.

- Specifying the primary operating parameters that affect the performance of HSBS. Separator geometry, properties of liquid and solid materials are considered. The velocities of fluidizing water as well as the oscillation of water flow are studied.

- Comparing the particle stratification in the presence of structured plates with that in an open column, with and without pulsating water. Identifying the positive effects of structured plates on coal particle separation.

- Validating of multiphase HSBS models, with and without, structured plates by inplant and laboratory data. 


\section{Chapter 2 Literature Review}

Gravity separation devices have been developed and used extensively in industry for many years. Separators, such as coal spirals and water only cyclones have been used in coal preparation plants to recover clean coal product from fine particle size ranges. This equipment usually gives low separation efficiency when compared with other coal cleaning separators in the plant. In coal preparation, spiral circuits suffer from having high density cut-

points. Water only cyclones tend to misplace coarse, high ash particles to clean coal product stream due to its relatively low classification efficiency; therefore, two-stage cleaning circuits are required. On the other hand, hindered-settling bed separators (HSBS), with higher separation efficiencies, are gaining more acceptances in the coal industry.

\subsection{Hinder-Settling Bed Separator (HSBS)}

The HSBS is a liquid-solid fluidized-bed separator in which the feed settles against an evenly distributed upward fluidizing water flow. Particles in such devices are separated primarily on the basis of particle density difference and to a lesser extent on particle size differences. HSBS are fine coal density separation equipment as shown in Figure 2.1. It has the ability to treat material in the size range $-2+0.25 \mathrm{~mm}$ at lower density cut-points than other available fine coal separators. The raw mineral particles enter the feed well and encounter either free or hindered-settling conditions, depending on the concentration of particles in the separator. The settling particles form a fluidized- bed above the fluidizing water injection point. The particles are then segregated or stratified based on the hindered- 


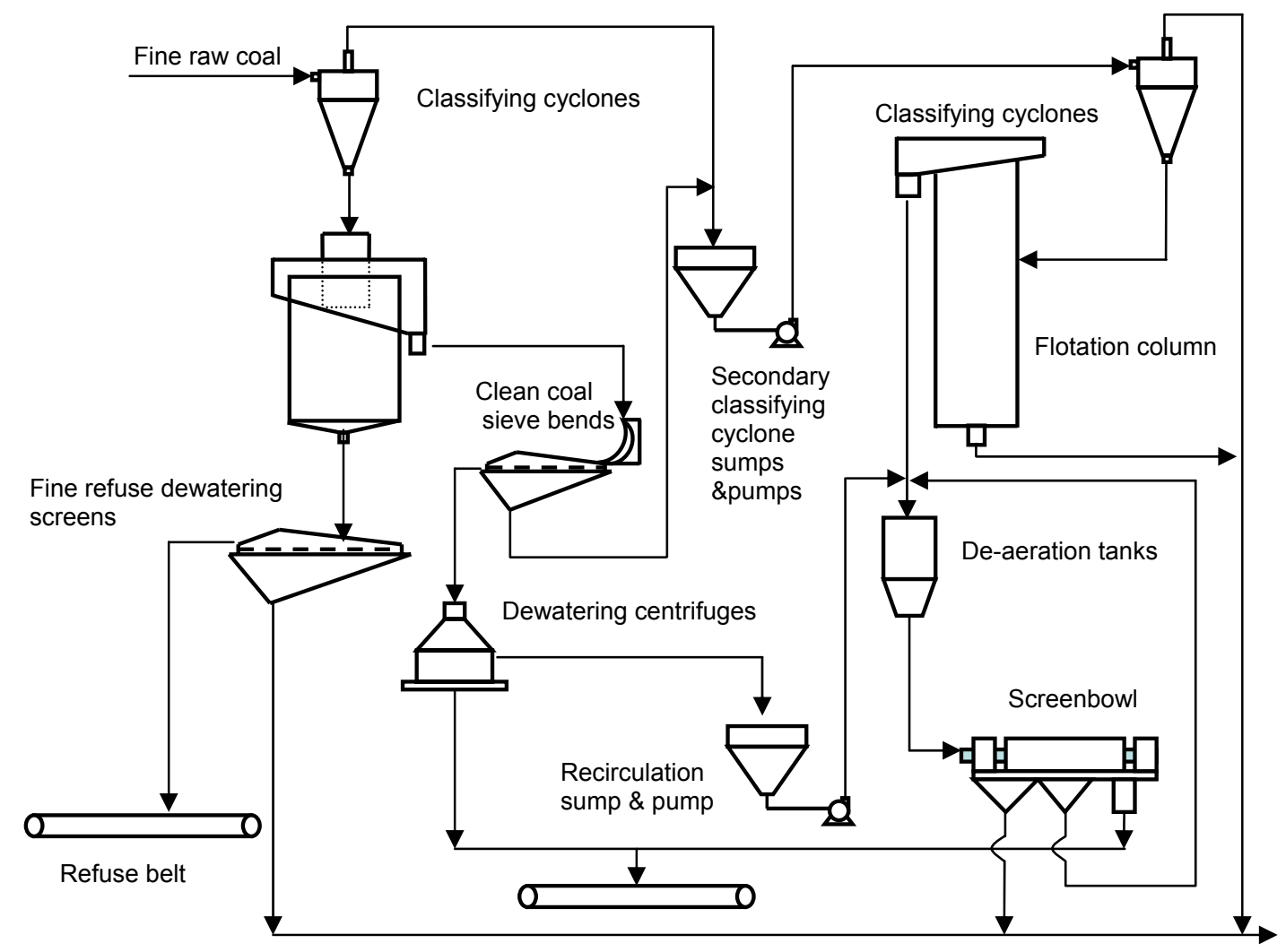

Figure 2.1 Schematic diagram of advanced fine coal cleaning circuit with HSBS

settling velocity of each particles.

HSBS have been used as size classification devices in the mineral industry for half a century. In this equipment, if the settling velocity of small particles is less than the fluidizing water velocity, these fine particles will report to the top of the fluidized-bed, while the coarse particles with higher settling velocities descend to the bottom of the fluidized-zone. However, if feed size distribution is within some limit, separation can be achieved largely 
based on density difference. Fine low-density particles report to the overflow lauder, while coarse and high-density particles reporting to the underflow discharge outlet.

HSBS offers a number of advantages including small footprint, adjustable cut-point, and stable operation over a broad range of throughput and high separation efficiencies. Applications of HSBS include: coal preparation (Reed et al., 1995; Honaker, 2000), mineral

sand benefication (Mankosa et al., 1995), and the recycling of chopped wires (Mankosa and Carve, 1995). The HSBS can also be utilized for many other industries, including: iron, tin, lead, zinc ore dressings, etc.

\subsection{Categories of HSBS}

The HSBS are primary categorized by the ways in which the material is discharged from the unit, and the way the slurry is introduced or whether if aeration is added. The well-known HSBS include Floatex (Litter, 1986) fluidized-bed classifier (or Floatex Density Separator) and the allflux ${ }^{\circledR}$ separator. There are three main commonly used types of devices: Centerfeeding HSBS, Cross-flow HSBS and Hydro-float HSBS.

\subsubsection{Center-Feeding HSBS}

A simplified schematic of a typical traditional HSBS with center feeding system is shown in Figure 2.2. Most HSBS utilize a center-tangential feeding pipe to minimize the disturbance by vertical fluidization and to introduce slurry material evenly over the system. The settling particles are hindered by upward fluidizing water and form a fluidized-bed above the fluidizing water injection level if particle hindered-settling velocities are equal to the upward fluidizing water rate. This fluidized-bed can also maintain a stable suspension density, which 


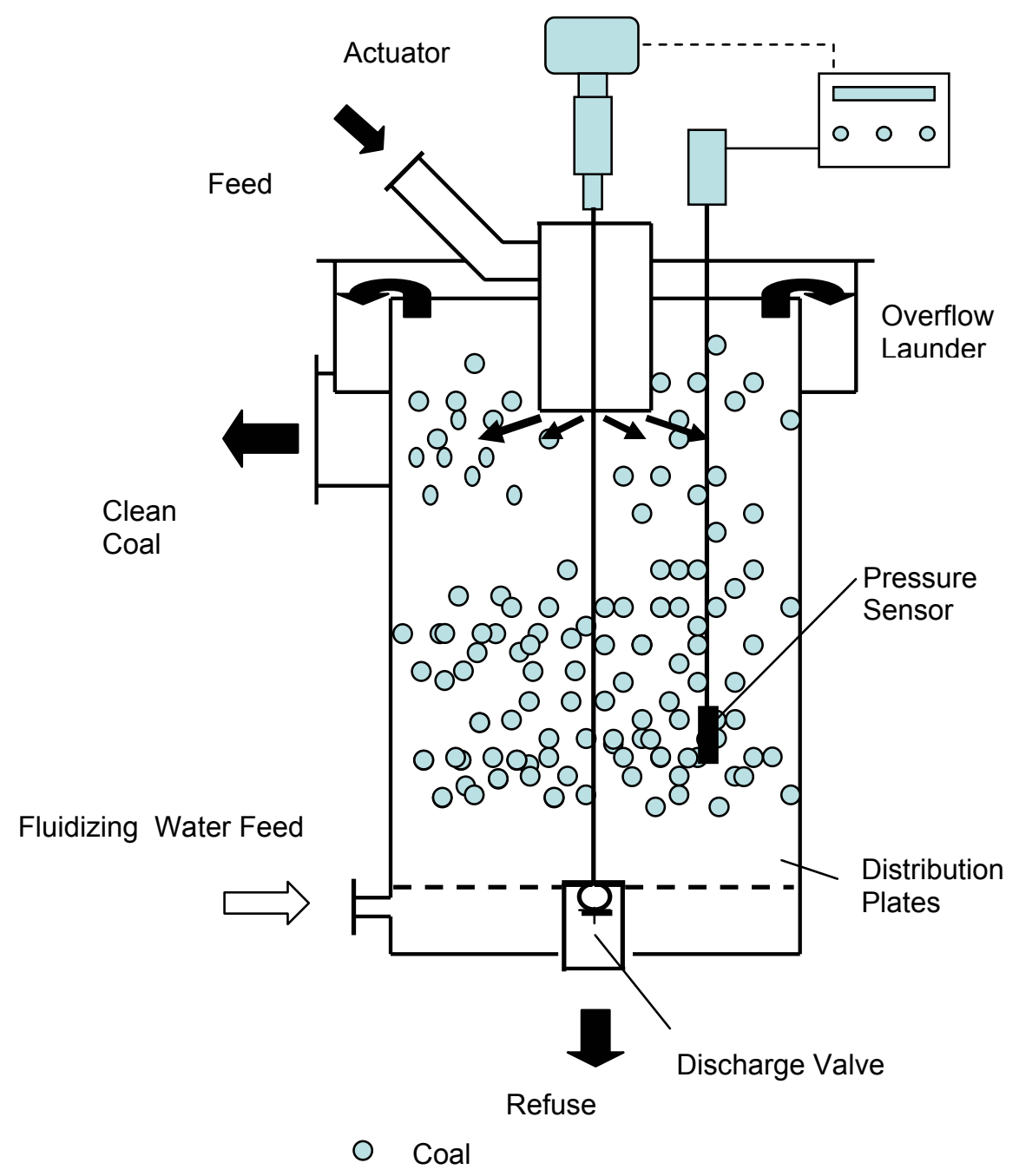

Figure 2.2 Schematic diagrams of center-feeding HSBS

helps to stop the penetration of a particle with less density. The region near the feeding pipe governs the entrance of new material into the cell and the initial segregation that occurs due to differential acceleration. The solid-liquid fluidized-bed is maintained within the lower part of the column. The pulp density and viscosity is significantly higher in this zone. The entering materials are separated based on hindered-settling velocities. The fine or low density 
particles will leave the fluidized-bed and report to the top of the fluidized-bed while the heavy and coarse particles descend to the bottom of the fluidized-bed. The fluidizing water can be injected by utilizing a distribution plate or pipe spargers. The typical representatives of this equipment are TBS, CDS and Hydrosizers (Little, 1986). Particles that settle through the fluidized-bed enter a dewatering cone and are discharged through an underflow control valve whose operation is actuated by the fluctuating rate of the fluidized-bed density.

\subsubsection{Cross-Flow HSBS}

The cross-flow separator is also a hindered-settling, counter-current hydraulic classifier that utilizes a liquid-solid fluidized-bed. As in center-feeding HSBS (Figure 2.3), the discharge speed of the underflow is controlled and a fluidized-bed is formed by solids settling against elutriation water (fluidization water) that is fed evenly across the entire crosssection of the unit. The feed is introduced in a horizontal level with a tangential flow at the top of the separator column. In order to reduce the feed flow, the feed stream enters a side feed well before flowing into the separation chamber. The feed stream then overflows the top of the device and enters the separation region. Heavy or coarse solid particles settle into the bottom while light and fine particles are carried out by horizontal water flow as the overflow product. This approach allows feed water to travel across the top of the unit and report to the overflow launder with minimal disturbance of the vertical fluidizing water within the separation chamber. The fluidizing water is injected through a set of pipe spargers. It is

reported that the Cross-Flow HSBS produced classification with higher efficiency and a lower density cutting point than that realized using a conventional center-centrifugal feed system (Kohmuench, 2000). This difference in separation efficiency may contribute to the elimination of disturbing water flow from the feed and thus reduced upward superficial water 


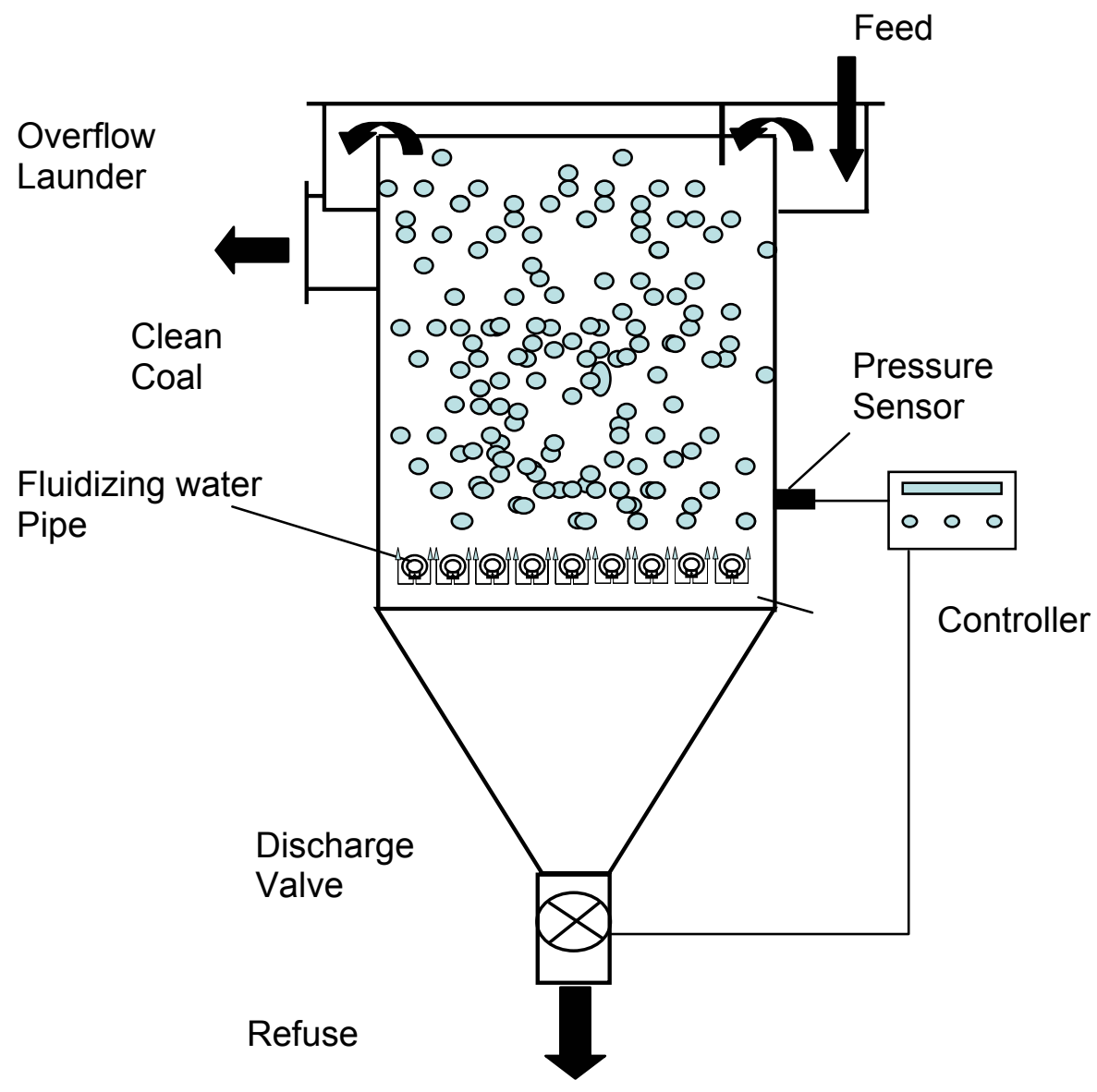

Figure 2.3 Schematic diagram of cross-flow HSBS

velocity in Cross-flow hindered-settling bed separator, though this hypothesis needs to be validated.

\subsubsection{Hydro-Float HSBS}

It should be noted that the HSBS shows a satisfactory density separation performance provided the feeding particle size range is within some limits. However, the fluidizing water velocity is usually low; these separators often suffer from the misplacement of coarse low density particles to the high density underflow if the feed has an overly broad size 
distribution. This leads to the accumulation of coarse, middle density particles that gather at the top of the fluidized-bed. These particles are too light to penetrate through the fluidizedbed, but are too heavy to be carried by the rising water into the overflow launder. Increasing the fluidizing water velocity seems to be a solution to convey the coarse, low-density solids to the overflow; however, the increasing upward flow will also elutriate the fine, high-density solids to the overflow launder, and this misplacement reduces the overall separation efficiency.

One solution is to introduce bubbles into the tanks to help carry the coarse middle density particle, if the low density particle is naturally hydrophobic or is made surface hydrophobic by being covered by collector agents. The idea of attachment of coal particles to a bubble is borrowed from the process of fine coal column flotation. A novel device known as the Hydro-Float separator for coal separation was developed based on this flotation mechanism. The Hydro-Float unit consists of a rectangular tank subdivided into an upper separation chamber and a lower dewatering cone. The device operates in a similar way as a traditional HSBS with the feed settling against an upward current of fluidizing water, however, the fluidized-bed is continuously aerated by injecting compressed air from the pipe spargers, with a small amount of frother into the fluidizing water. The air bubbles become attached to the hydrophobic particles within the fluidized-bed, thereby getting a low particle-bubble bulk density. The mineral particles may be naturally surface hydrophobic or made surface hydrophobic by the addition of collectors. The lighter bubble-particle aggregates rise to the top of the denser fluidized-bed and overflow the top of the separation chamber. In general, the Hydro-Float separator utilizes the combination of flotation and fluidizing effects in the same tank to get a higher recovery of coarse coal particles. 


\subsection{Advances in HSBS Design}

Conventional liquidized bed separators, including the HSBS, have a direct one to one relationship between concentration and the fluidizing water velocity. In order to obtain a satisfactory density separation, the fluidizing water velocity must be maintained at the lowest possible level. This limits the throughput of HSBS. Increasing water flow can increase the throughput but generally destroys the density separation. At a high fluidizing water velocity level, all particles might be elutriated and no separation happens. Fluctuations in fluidizing water velocity can also cause fluctuations in the fluidized-bed density and further affect density separation. The addition of structured plates is one solution to this problem.

\subsubsection{HSBS with Parallel Inclined Plates}

Installation of baffles or structured plates in a HSBS can greatly improve the separation performance and increase throughput. Galvin et al. (2002) have investigated the interaction of a set of parallel inclined plates and a fluidized suspension in the Reflux ${ }^{\circledR}$ classifier (Figure 2.4). One advantage of this equipment is that Reflux ${ }^{\circledR}$ can maintain a stable suspension concentration with the help of the inclined plates while using a broad range of possible fluidizing water velocities. It was postulated that, at high solid feed rate, the extra particles can reach the upward facing inclined plates and slide downward to the fluidized-bed zone below the inclined plates. The flow patterns and particle separation mechanism involved in the sliding along the plate surfaces are not clearly described in the literature. 


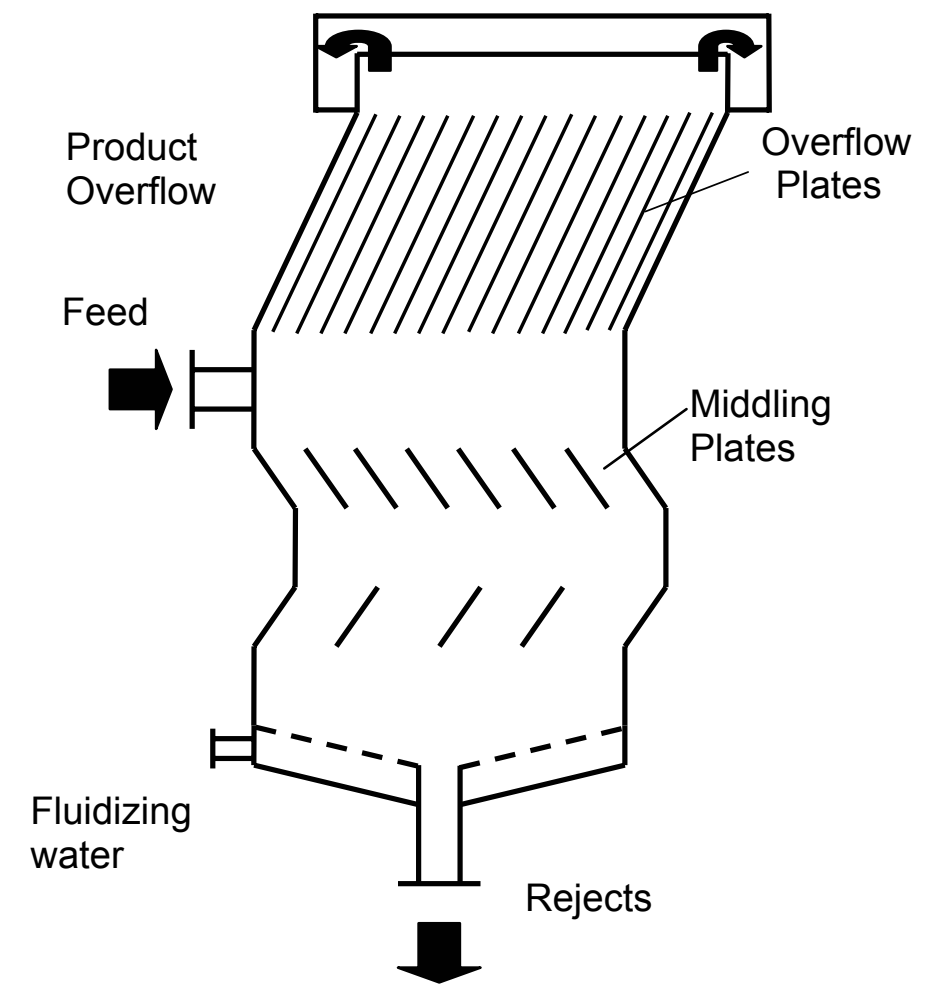

Figure 2.4 Schematic representation of the Reflux classifier

(Galvin and Dorodchi, et al., 2002)

\subsubsection{HSBS with Structured Plates}

From experimental work, it was found that the column jig (Figure 2.5) shows a good separation for fine particles (Dai, 1999; Yang and Bozzato, 2000; Peng, et al., 2002). It consists of a packed column filled with a helical static mixer and a pulsating device for generating jigging actions within the column. By adjusting the frequency and amplitude of pulsation as well as the fluidizing water velocity, the feed particles can be separated by density or size. The operation difference between HSBS and this jigging separator lies in that there is an extra pulsating water flow in jigging process while only a stable fluidizing water flow exists in an open hindered-settling column. The inclusion of structured plates and 


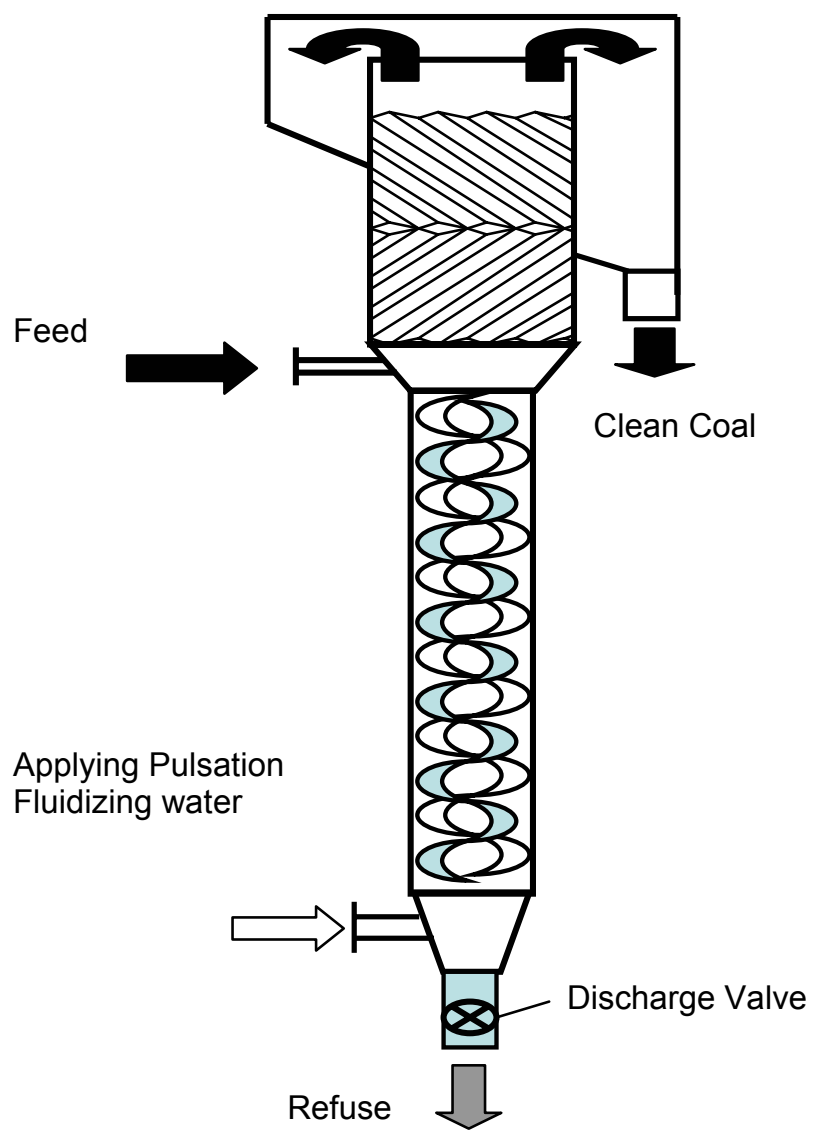

Figure 2.5 HSBS with structured plates

the addition of water pulsation in hindered-settling will help to extend its lower separation size limit. It has attained a number of promising separation results for various minerals; however, little is still known about its many interacting factors that affect fine particle separation. The density separation mechanism in a pulsating flow in the presence of structured plates in the HSBS is still not clear. Thus, there is a need to determine the effect of these factors on density separation. Computer modeling with incorporation of pulsation flow 
characteristics and structure plates in the HSBS is an efficient approach to understand the phenomena.

\subsection{Modeling of HSBS}

A better understanding of the phenomena of hindered-settling is fundamental to describing the separation processes that occur in gravity separation and classification devices. With such an understanding, it should be possible to develop an accurate process model that is applicable to broad range of feeds, and in turn optimizes process flow-sheets or even

develop new or improved separation processes. A good description of hindered-settling velocity of a particle should be applicable to a suspension that consists of a broad range of particle sizes and densities. However, in the past, most hindered-settling models have concentrated on estimating hindered-settling velocities and have involved only a simple suspension consisting of either constant density distribution or uniform size distribution (AlNaafa and Selim, 1992; Davis and Gecol, 1994).

\subsubsection{Hindered-Settling Velocities}

\subsubsection{Free Settling}

For an isolated sphere, the terminal velocity, $\mathrm{U}_{\mathrm{t}}$, in the laminar flow is:

$$
\mathrm{U}_{\mathrm{t}}=\frac{\left(\rho_{\mathrm{p}}-\rho\right) g \mathrm{~g}^{2}}{18 \mu}
$$

At higher Reynolds numbers, Zigrang and Sylvester (1981) used the following empirical equation: 


$$
U_{t}=\frac{\mu}{\rho d}\left[\left(\frac{14.51+\left(g\left(\rho_{s}-\rho\right) \rho\right)^{0.5} 1.83 d^{1.5}}{\mu}\right)^{0.5}-3.81\right]^{2}
$$

where $\rho$ is the density of the liquid, $\rho_{\mathrm{s}}$ is the density of the solid particle, $\mu$ is the viscosity of the liquid, and $\mathrm{d}$ is the diameter of the particle. In a HSBS, seldom do particle have a free settling condition; instead, the particles are separated in a pulp with high solids concentration.

\subsubsection{Hindered-settling}

Hindered-settling occurs when the settling of a particle in a liquid suspension is affected by the presence of nearby particles. With increases in solid concentration, the distance between particles is reduced such that the drag force created by the settling particles changes the surrounding liquid flow and affects the movement of nearby particles (Mirza and Richardson, 1979). At high solids concentration, entrapment and misplacement of particles will dominate, thereby increasing the en-mass settling, which is independent of particle size and density. The terminal settling velocity of a particle needs to be adjusted to account for the presence of other particles in a solid suspension. The adjustment can be made by including the solid volume fraction, apparent viscosity or suspension density in the calculation (Richardson and Zaki, 1954, and Galvin et al., 1999).

When the test particle resides in a suspension, it is appropriate to refer to the slip velocity, $\mathrm{U}_{\text {slip }}$ and to account for the presence of other particles (Richard and Zaki, 1954):

$$
\begin{aligned}
& \mathrm{U}_{\text {slip }}=\mathrm{U}_{\mathrm{s}}-\mathrm{U}_{\mathrm{f}} \\
& \mathrm{U}_{\text {slip }}=\mathrm{U}_{\mathrm{t}}(1-\phi)^{\mathrm{n}-1}
\end{aligned}
$$


where $\mathrm{U}_{\mathrm{s}}$ is particle velocity and $\mathrm{U}_{\mathrm{f}}$ is liquid velocity, $\phi$ is the solid volume fraction. Lockett and Al-Habbooby $(1973,1974)$ extended this equation to cover more than one particle species and reported a value of 4.65 for $\mathrm{n}$ at low Reynolds number. Garside and Al-Dibouni (1977) suggested $\mathrm{n}=5.1$ for low Reynolds numbers, and reached a general expression:

$$
\mathrm{n}=\frac{5.1+0.27 \mathrm{Re}^{0.9}}{1.0+0.1 \operatorname{Re}^{0.9}}
$$

The terminal settling velocity can also be corrected empirically using effective viscosity or apparent viscosity. Masliyah (1979) utilized the following expression in laminar flow:

$$
v_{t}=\frac{g d^{2}\left(\rho_{s}-\rho_{f}\right)}{18 \eta_{f}} \alpha_{f} F(\alpha)
$$

where $g$ is the force due to gravity, $d$ is the diameter of the particle, $\rho_{s}$ is the density of the solids, $\rho_{\mathrm{f}}$ is the density of the fluidizing medium, $\alpha_{\mathrm{f}}$ is the suspension voidage $(1-\phi)$, and $\eta_{\mathrm{f}}$ is the viscosity of the fluid. The term $F(\alpha)$ describes a function that accounts for particle concentration. In non-Stokes flow, the corrected expression is listed below:

$$
v_{t}=\frac{g^{2}\left(\rho_{s}-\rho_{\text {susp }}\right)}{18 \eta_{f}\left(1+0.15 \operatorname{Re}^{0.687}\right)} \alpha_{f} F(\alpha)
$$

where $\eta_{\text {susp }}$ is the apparent viscosity of the suspension. Richardson and Zaki (1954) defined $\mathrm{F}(\alpha)$ as $(1-\phi) \beta$ where $\beta$ is an unknown function of particle size and shape. For transitional flow regimes normally found in hindered-bed separator applications, $\beta$ can be determined as seen below:

$$
\begin{array}{ll}
\beta=4.36 \mathrm{Re}^{-0.03} & \text { for } 0.2<\operatorname{Re}<1.0 \\
\beta=4.4 \mathrm{Re}^{0.1} & \text { for } 1.0<\operatorname{Re}<500
\end{array}
$$


Another commonly accepted form of this function for transitional flow regimes comes from Barnea and Mizrahi (1973) where:

$$
F\left(\alpha_{f}\right)=\left[1+\left(1-\alpha_{f}\right)^{1 / 3} \exp \frac{5\left(1-\alpha_{f}\right)}{3 \alpha_{f}}\right]^{-1}
$$

Mondal (1997) lists several other expressions in his work for hindered-settling velocities, including those derived by Concha and Almendra (1979). However, these settling equations are not valid for non-transitional flows. Work has also been conducted by Brauer et al. (1973) shows that hindered-settling velocity depends on the free settling velocity of a particle in a narrow size and density class (i), the particle size, and the distance to any other particles. According to Brauer et al. (1973) the hindered-settling velocity $\left(\mathrm{v}_{\mathrm{hi}}\right)$ can be calculated as:

$$
\mathrm{V}_{\mathrm{hi}}=\mathrm{V}_{\mathrm{oi}} \mathrm{k}_{\mathrm{if}} \mathrm{k}_{\mathrm{ic}}
$$

where the parameter $k_{\text {if }}$ is a fluid counter flow factor which addresses the displacement of water by settling particles. The parameter $\mathrm{k}_{\mathrm{ic}}$ is another factor that addresses turbulence caused by clusters of settling particles. All these factors are measured or empirically determined.

Asif (1997) and Galvin (1999) emphasized the empirical dependence on the suspension density, arriving at

$$
\mathrm{U}_{\text {slip }}=\mathrm{U}\left(\frac{\rho_{\mathrm{p}}-\rho_{\text {susp }}}{\rho_{\mathrm{p}}-\rho}\right)^{\mathrm{n}-1}
$$


Although the preceding equations have some success in the description of specific systems, it is questionable that there exists a satisfactory description of hindered-settling that is reliable for general use in mineral processing.

\subsubsection{Existing HSBS Models}

An empirical model for particles settling in a cone classifier was built by Kojovic and Whiten (1993) to explore the effects of geometry and operating parameters on separation. Mackie et al. (1987) developed a hybrid physical-empirical separation model, which is based on settling theory, to describe the operation of a Stokes hydrosizer. Smith (1991) developed a mathematical model of an elutriator using differential settling velocities of binary mixtures proposed by Lockett and Al-Habbooby (1973). The most popular models are population balance models used by Honaker and Modal (2000) and Convection-Diffusion Model by Kim et al. (2003).

\subsubsection{Dynamic Population Balance Model}

The design and reliable scale-up of HSBS requires a good model to describe the separation mechanism in the separating process. However, review of the literature reveals that very little information has been published in the area. The complexity in the operation of the classifiers is due to the extensive interactions of the operating parameters and the resulting impacts on separation performance. Most models usually ignore the interaction among different phases and try to build a macroscopic mass balance over separation with an assumption that the liquid flow is uniform along the separator column.

The most commonly used model is called the population balance model (Mondal, 1997; Swanson, 1999). Mondal (1997) utilizes the hindered-settling velocities equation to show 
significant upgrading when processing fine coal slurry using a Floatex Density Separator. Swanson (1999), on the other hand, utilized a semi-empirical process that was able to show simulated consistency with 50 sets of test data. Both models demonstrate impressive results. However, these models are not universally applicable.

A mathematical dynamic population balance model has been developed by Kohmuench (2000) to help understand separation in a Cross-Flow HSBS. This model utilizes general equations for hindered-settling in transitional flow regimes to accurately predict overflow and underflow partitions, particle size distributions, and component recovery data. Input data include feed rate, percent feed solids (by mass), feed size distribution (up to 9 size fractions), density of components in the feed stream (up to 2 components), fluidizing water rate, and underflow discharge rate.

This model considers the effect from the presence of multi-species variations in particle size and density, and an upward fluid flowing against the settling of particles. This upward flow is a function of particle volume. The division and definition of computation zones in a Cross-Flow separator are shown in Figure 2.6.

The Cross-Flow separator is principally constructed as a series of well-mixed zones. These zones represent three distinct sections that have dissimilar mixing patterns and flow regimes. Therefore, each section must be modeled accordingly. The three primary sections include the feed inlet, the fluidized-bed, and the underflow areas. The model was constructed using Microsoft Excel. The advantages of using Excel are declared to include instant graphing of results, and more importantly, ease of troubleshooting. However, there are some limitations existing with this approach: 


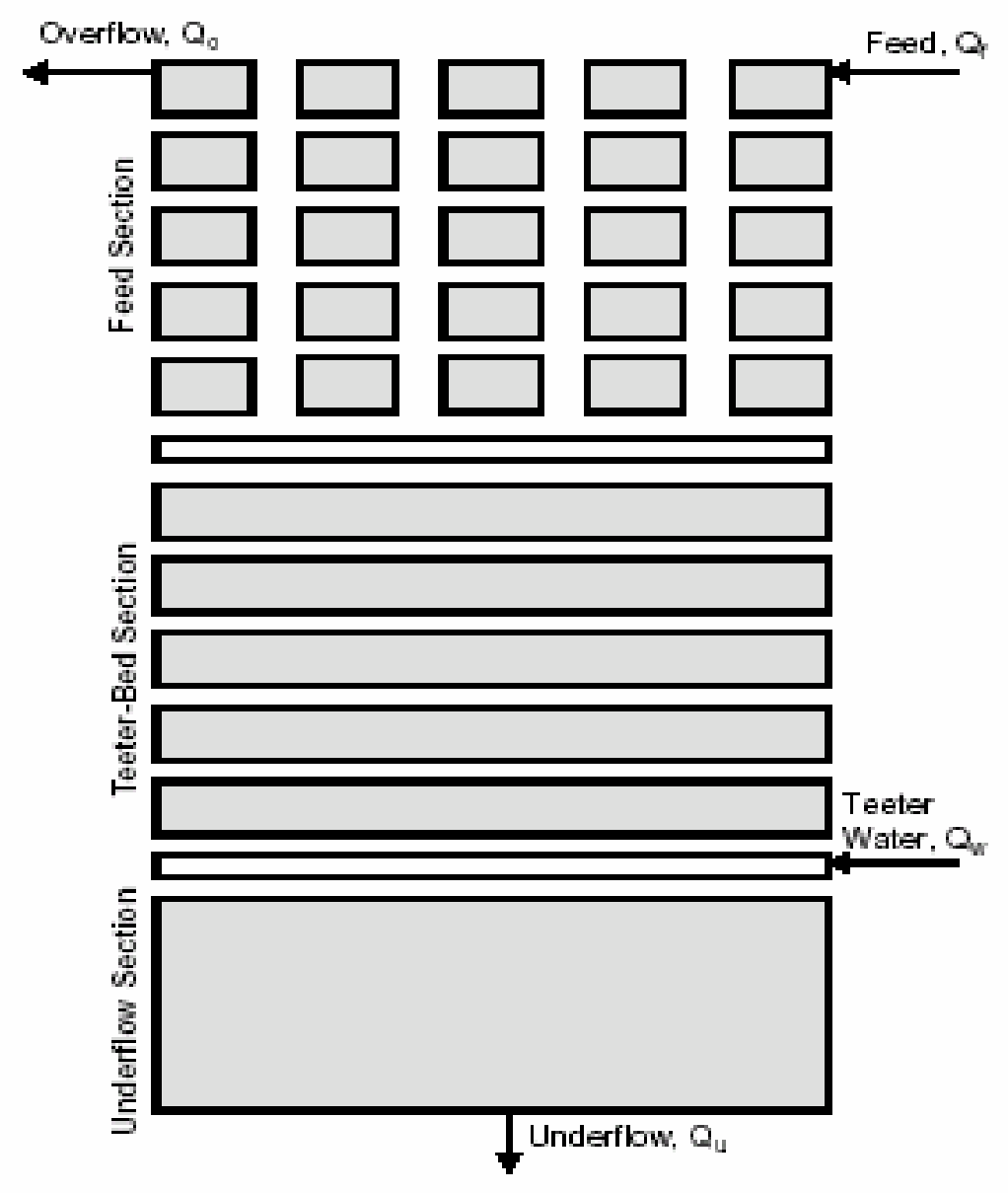

Figure 2.6 Schematic depicting the primary divisions and flows for the cross-flow HSBS using the population balance model (Kohmuench, 2000).

- Arbitrarily dividing the domain into a few regions, and the uniform fluid field in each region are assumed.

- Although the overall mass conservation (steady-state flow) is maintained, not much understanding of the dynamic separation phenomena is provided.

- No details about the movement and separation of the particles in the horizontal direction are revealed. 
- There are limitations in dealing with size and density distributions. In the above application, it only deals with feed which has up to 9 size fractions and up to 2 density fractions only.

- The model is difficult to use for HSBS with a complex geometry, where a complicated flow can't be assumed to be uniform.

\subsubsection{Convention-Diffusion Model}

Kim et al. (2003) proposed a dynamic type model, which is based on the general convection-diffusion equation, to describe the hindered-settling conditions. The conventiondiffusion equation is given by

$$
\frac{\partial \varphi(\mathrm{x}, \rho, \mathrm{z}, \mathrm{t})}{\partial \mathrm{t}}=\mathrm{D} \frac{\partial^{2} \varphi(\mathrm{x}, \mathrm{v}, \mathrm{z}, \mathrm{t})}{\partial \mathrm{z}^{2}}-\frac{\partial(\mathrm{V}(\mathrm{x}, \rho, \mathrm{z}, \mathrm{t}) \varphi(\mathrm{x}, \rho, \mathrm{z}, \mathrm{t}))}{\partial \mathrm{z}}
$$

where $\phi$ is the volume fraction of particles of size $x$ to $x+d x$ with a density of $\rho$ to $\rho+d \rho$ in the element of $\mathrm{z}$ and $\mathrm{z}+\mathrm{dz}$ at time $\mathrm{t}$; $\mathrm{D}$ is the particle diffusion coefficient.

In this model, the hindered-settling particle velocities are calculated by an empirical equation:

$$
\mathrm{U}=\frac{20.52 \mu_{\mathrm{f}}}{\mathrm{d} \rho_{\mathrm{P}}} \mathrm{f}_{1}(\phi)\left\{\left[1+0.0921\left(\frac{\mathrm{d}^{3}\left|\rho_{\mathrm{s}}-\rho_{\mathrm{p}}\right| \rho_{\mathrm{p}} \mathrm{g}}{0.75 \mu_{\mathrm{f}}^{2}}\right)^{1 / 2} \mathrm{f}_{2}(\phi)\right]^{1 / 2}-1\right\}^{2}
$$

where

$$
\begin{aligned}
& f_{1}(\phi)=\frac{\left(1+0.75 \phi^{1 / 3}\right)(1-\phi)\left(1-1.47 \phi+2.67 \phi^{2}\right)^{2}}{(1-1.45 \phi)^{1.83}\left(1+2.25 \phi^{3.7}\right)} \\
& f_{2}(\phi)=\frac{\left(1+2.25 \phi^{3.7}\right)(1-1.45 \phi)^{1.83}}{\left(1+0.75 \phi^{1 / 3}\right)(1-\phi)\left(1-1.47 \phi+2.67 \phi^{2}\right)}
\end{aligned}
$$


where $f_{1}(\phi)$ and $f_{2}(\phi)$ are empirical functions that account for the effects of solid concentration, $\phi$ is the solid volume fraction, $\mu_{\mathrm{f}}$ is fluid viscosity, $\mathrm{g}$ is gravity acceleration, $\rho_{\mathrm{s}}$ is particle density, and $\rho_{\mathrm{p}}$ is pulp density.

A finite-difference solution scheme is used to solve the above equations. The hinderedsettling column is divided into several elements, each having unique combination of an average particle diameter and an average density. At each time step, new particles are added and products are removed continuously. The model is used to account for the operating and design variables of the column including solids feed velocity, fluidizing water rate, bed height, feed location and column height. While the mass balance in each element at each time step is ensured, the wall effect and particle collision are ignored in this model.

\subsection{Challenges in Modeling HSBS}

Some of the key features of the HSBS are as follows: a) Less maintenance; b) easy to control; c) low operating costs; and d) high efficiency that consistently provides high solids handling capacity. While the HSBS have brought various benefits and advantages to mineral processing, many issues have been identified with the implementation and use of this technology. These issues have been observed in both continuous and batch tanks. The fundamental concepts of HSBS have been well described in previous publications. However, complexity in the operation of the equipment is realized due to the extensive interactions of the operating parameters and the resulting impacts on separation. The primary concerns are related to the following aspects:

- A reliable model used for scaling-up of the HSBS is still unavailable. Laboratory scale and pilot scale tests are still needed and expensive. 
- Although HSBS have a good separation if particle size is within a given size range, if the particle is too fine, the separation becomes worse. The inclusion of baffles or packing has improved the fine particle separation, but the positive contribution from structured plates to separation is still not clear. The hydrodynamic behavior of the structured plates in separation need to be explored. The current models can not simulate cases which incorporate inclined plates or structured plates.

- The particle stratification is a typical multiphase flow phenomenon involving the motion of particles, inter-particle collisions, wall-particle collisions and liquidparticle coupling. An appropriate model incorporating particle collisions for multiphase flow in HSBS needs to be determined.

- The optimal design of the HSBS needs to determine all of the main parameters that affect separation. These factors include column and packing geometry, feed material properties, liquid properties, inlet fluidizing water velocity, magnitude and frequency of water oscillation, etc. These parameters are not very easy to test experimentally.

\subsection{Application of Multiphase Modeling in Mineral Processing}

\subsubsection{Modeling of Jigging}

Jigging is an important process in which mineral particles move in pulsating water resulting in a stratification of particles of different gravities and obvious size differences. Jigging is widely applied in coal preparation because of its simple operation, simple construction of equipment and high capacity. The actual particle jigging happens in a complicated multiphase flow system. Different particles are exposed to different forces in the 
liquid and this results in different particle trajectories based on the fluid velocity profile and the mineral properties.

Many models have been used to try to understand the jigging process. These main models can be roughly be classified as following:

- Potential energy theory (Mayer, 1964; Tavares and King, 1995)

- Energy dissipation theory (Jinnouchi and Kawashima, 1984)

- DEM (discrete element method) approach (Srinivasan et al., 1999; Mishra and Mehrotra, 2001; Beck and Holtham, 1993)

These models can be classified basically into two groups of models: potential energy model and discrete element models (DEM). Mayer (1964) proposed his potential energy model. Based on this model, the potential energy difference between un-stratified and stratified particle bed is the driving force to cause separation. While this model is widely accepted to describe the macroscopic behavior of the particle bed and many modifications of this model have been made, the potential model still can not be applied directly to the real jigging operation. This model can only do qualitative explanation of the stratification process, it can not be used to describe the real behavior of jigging operations. Tavares (1995 and 1999) made a modification of Mayer to simulate the jigging of binary-sized feeds to overcome the limitation of Mayer's model that can only deal with a uniform sized feed. However, it is still difficult to extend this model to the jigging of particles of widely varied size distribution.

In order to get a quantitative analysis of the jigging process, Mishra et al. (1998) and Beck and Holtham (1993) used a microscopic model in which the two-dimensional discrete element method (DEM) is adopted. Srinivasan et al. (1999) investigated a three-dimensional 
DEM model to simulate stratification of particles in jigs. Misha and Meharotra (2001) improved this 3-D DEM model by incorporating a new correlation of drag force in order to take account of bed porosity. They validate their model against experimental data, although the correlation is not satisfactory.

While the microscopic model is more specific and the macroscopic model is more basic, each of these kinds of models has its drawbacks in modeling the jigging process. For the microscopic model, the exact determination of the forces acting on particles is always a difficult job, especially when the solids concentration is high and particle collisions are unavoidable. For the macroscopic model, its interpretation can hardly tell details of the separation process and thus reduces its usefulness.

With the DEM approach, not only optimal operating parameters such as amplitude and frequency are determined in order to get the best performance of jigging, but also the different pulsating waveforms of liquid in jigging can be compared and selected (Armstrong, 1963; Beck and Holtham, 1993; Sinivansan, 1999). All of the above models still use an assumed uniform fluid field and do not consider the effect of possible non-uniform fluid velocity on particle drag forces. While the DEM model of the particle interaction problem is completed, the model for the analysis of the fluid is still under progress. The existing nonuniform particle concentration (bed porosity distribution) will also change the fluid velocity field.

In general, existing theories which are derived from potential energy theory, can illustrate macroscopic behavior. However, they provide only a qualitative explanation of the jigging process and contribute little to designing of jigging equipment. In DEM models, the motion of individual particles can be traced as time progresses and the macroscopic behavior 
of whole mineral assembly can be calculated by summarizing individual parameter components. In this way, many sub-processes of jigging such as density distribution, instant potential energy changes, size distribution, etc. can be quantitatively determined. Usually, an idealized fluid behavior is assumed in these models and the damping effect of movement of particles on liquid phase motion is ignored. It is improper to apply this model in explanations of jigging when particles are densely distributed or stratification of particles in a baffled column where fluid motion is complicated by the presence of structured plates.

\subsubsection{Application of CFD in Multiphase Modeling}

Computational Fluid Dynamics (CFD) is increasingly being used to analyze the flow and performance of process equipment, such as chemical reactors, fluidized-beds, combustion systems, spray dryers, pipeline arrays, heat exchangers, and other equipment. There is also a trend for CFD to be used in research for separation equipment in the area of mineral processing, such as: stirred tanks, cyclones, mechanical flotation machines, filter and bubbly column, etc. CFD allows for an in-depth analysis of the fluid dynamics of this system and therefore, better design of this equipment. In many cases, this results in improved performance, better reliability, more confident scale-up, improved product consistency, and higher capacity.

This study will explore the application of CFD approaches in water based gravity separators which are widely used in mineral processing. The interaction between solid particles and laminar or turbulent flows has been a research topic of both fundamental importance and practical interest. An aspect in which we are particularly interested here relates to the effect on particle stratification arising from interactions of particles with liquid 
in hindered-settling tanks or columns at where the flow velocity is complicated and a simple arbitrary flow pattern in numerical simulation is obviously not applicable.

Numerical calculations have been made of particles dispersion and particle-fluid interactions in turbulent flows for many years. For engineering problems, two approaches based on the Reynolds-averaged Navier-Stokes equations are commonly applied, namely the two-fluid or Euler-Euler approach and the Euler-Lagrange method. These two methods are summarized here and details of the Euler-Lagrange model will be given in Chapter 3 .

In the Euler-Euler approach both liquid and solid phases are considered as interacting continua. In turbulent flows, the fluid-particle interaction terms are associated with sophisticated modeling approaches (Rizk \& Elghobashi, 1989, and Simonin et al., 1993). The consideration of a particle size distribution requires the solution of a set of basic equations for each size class to be considered. Hence, the computational effort increases with the number of size classes. This method is however preferable for discontinuous and dense twophase flows. For example, flows found in fluidized-beds with uniform particles. In general, this approach could be used in cases where dense solid or gas two phase flows exists and all parameters are defined and used as average variables. However, this model ignores the discrete nature of particles and it is obvious that it is not applicable to simulation of particle separation in HSBS where particles have a broad size distribution and density composition.

The second approach, the Euler-Lagrange approach, is to calculate the fluid flow in an Euler continuous frame with the particles being moved individually in Lagrangian frame coordinates. The Euler-Lagrange approach is applicable to the problem of the dispersed phases and especially accounts for the discrete nature of the individual particles. Generally, the particles are considered as point particles, i.e. the finite dimension of the particles is not 
considered and the flow around the individual particles is not resolved. Since the number of real particles in a flow system is usually too large to allow a tracking of all particles, the trajectories of computational particles (i.e. parcels) which represents a number of real particles with the same properties (i. e. size and density) are calculated. Local average properties such as dispersed particle density and velocity are obtained by ensemble averaging. Statistically reliable results for each computational cell might require the tracking of a large number of particles, depending on the considered flow and size of domain. In spite of this shortcoming, the advantage of this method is that physical effects influencing the particle motion, such as particle-turbulence interaction, particle-wall collisions, and collisions between particles can be modeled on the considered parcels by sampling the size of the injected particles from a given distribution function. 


\section{Chapter 3 Multiphase Flow Model Fundamentals}

CFD tools provide comprehensive modeling capacities for a wide range of incompressible and compressible, laminar and turbulent fluid flow problems. For modeling the multiphase phenomena in HSBS, the physical models as well as their corresponding governing equations are employed. In this chapter, models for the liquid phase flow and for the solid particles in the physical separation processes are identified specifically for HSBS.

\subsection{Basic Physical Models for Liquid Phase}

The basic models for multiphase flows in HSBS include continuity, momentum equations for liquid phase, and momentum equations for the solid phase. Collision models for particles are so important that they are described in Chapter 4 respectively.

\subsubsection{Conservation of Mass}

The general form of the mass conservation equation or continuity equation for a continuous flow is

$$
\frac{\partial \rho}{\partial t}+\frac{\partial}{\partial x_{i}}\left(\rho u_{i}\right)=S_{m}
$$

where

$$
\begin{aligned}
& \rho=\text { density, } \mathrm{kg} / \mathrm{m}^{3} \\
& \mathrm{u}_{\mathrm{i}}=\text { the velocity in the i direction, } \mathrm{m} / \mathrm{s} \\
& \mathrm{S}_{\mathrm{m}}=\text { the source term of mass, } \mathrm{kg} / \mathrm{m}^{3} \mathrm{~s}
\end{aligned}
$$


Eq.(3-1) is valid for incompressible as well as compressible flows. $\mathrm{S}_{\mathrm{m}}$ is equal to zero for incompressible flow. Because the flow in the HSBS involves species mixing and removing, the species mass and momentum conservation equations must be solved.

\subsubsection{Conservation of Momentum}

For a single viscous flow phase, the momentum equation (Navier-Stokes equation) takes the form

$$
\frac{\partial}{\partial t}\left(\rho u_{i}\right)+\frac{\partial}{\partial x_{i}}\left(\rho u_{i} u_{j}\right)=-\frac{\partial p}{\partial x_{i}}+\frac{\partial \tau_{i j}}{\partial x_{j}}+\rho g_{i}+F_{i}
$$

where

$$
\begin{aligned}
& \rho=\text { the density, } \mathrm{kg} / \mathrm{m}^{3} \\
& \mathrm{u}=\text { velocity, } \mathrm{m} / \mathrm{s} \\
& \mathrm{p}=\text { pressure, } \mathrm{N} / \mathrm{m}^{2} \\
& \mathrm{~F}_{\mathrm{i}}=\text { the component of body force in i direction } \\
& \mathrm{g}_{\mathrm{i}}=\text { the acceleration in i direction, }{\mathrm{m} / \mathrm{s}^{2}}^{2} \\
& \tau_{\mathrm{ij}}=\text { the shear tensor which is given by } \\
& \tau_{\mathrm{ij}}=\left[\mu\left(\frac{\partial \mathrm{u}_{\mathrm{i}}}{\partial \mathrm{x}_{\mathrm{j}}}+\frac{\partial \mathrm{u}_{\mathrm{j}}}{\partial \mathrm{x}_{\mathrm{i}}}\right)\right]-\frac{2}{3} \mu \frac{\partial \mathrm{u}_{1}}{\partial \mathrm{x}_{1}} \delta_{\mathrm{ij}}
\end{aligned}
$$

with

$$
\begin{aligned}
\mu & =\text { the viscosity } \\
\delta_{\mathrm{ij}} & =\text { the kronecher delta }
\end{aligned}
$$

here, $\mathrm{i}, \mathrm{j}, 1$ are the three orthogonal directions. 


\subsection{Turbulence Model}

\subsubsection{Flow Regimes}

Viscous flows are classified into laminar or turbulent regimes based on of their internal flow structure. In the laminar regime, particles move in laminar or layers. Flow structure in the turbulent regime is characterized by random motion within space and time, with the three-dimensional motion of the fluid particles superimposed on the mean motion. Whether the flow in a HSBS is laminar or turbulent depends on the given conditions, such as fluidizing water velocity, viscosity of pulp etc. Considering the flow through a duct such as the feed inlet, the Reynolds Number can be used to characterize the flow

$$
\mathrm{Re}=\frac{\rho U L}{\mu}=\frac{\mathrm{UL}}{\gamma}
$$

where

$$
\begin{aligned}
& \rho=\text { the density of the mixture solid and liquid, } \mathrm{kg} / \mathrm{m}^{3} \\
& \mathrm{U}=\text { the mean velocity of the flow, } \mathrm{m} / \mathrm{s} \\
& \mathrm{L}=\text { the characteristic length of the duct, } \mathrm{m} \\
& \mu=\text { the kinetic viscosity, } \mathrm{kg} / \mathrm{m}-\mathrm{s} \\
& \gamma=\text { the viscosity of the fluid, } \mathrm{m}^{2} / \mathrm{s}
\end{aligned}
$$

The energy intensity required for the solid particle stratification will result in a high teetering water velocity. Also the movement of particles in the fluid will cause a disturbance in the surrounding flow. These complicated phenomena make flow in the HSBS turbulent. 


\subsubsection{Selection of Turbulent Models}

Turbulent flows are characterized by fluctuating velocity fields. The fluctuations are too computationally extensive to simulate directly in practical engineering calculation. Instead, the exact governing equations can be time-averaged, ensemble-averaged, or manipulated to remove the small scales, resulting in equations that are computationally less extensive to solve. However, the modified equations contain additional unknown variables, and thus the turbulence models are needed to determine these variables in terms of known quantities.

Generally, both the Reynolds-averaged approach and the Large Eddy Simulation (LES) approach can be employed to transform the Navier-Stokes equations in such a way that the small scale turbulent fluctuations do not have to be directly simulated. However, here, only the Reynolds-averaged approach is considered for the simulation of the hindered-settling tanks based on the following three factors: (1) the mean flow in the tank is steady during separation; thus, using the Reynolds-averaged approach greatly reduces the computational efforts, (2) large computer resources are required to resolve the energy-containing turbulent eddies if LES approach is used, (3) the Reynolds-averaged approach has been proven to be suitable for industrial fluid simulations, such as industrial stirred tank, flotation cell, bubble columns and thickeners.

The literature on applications of computational fluid dynamics (CFD) to multiphase flows shows that the standard k- $\varepsilon$ model and the Baldwin-Lomax model are widely used for multiphase flow calculations and predictions. 


\subsubsection{Standard k- $\varepsilon$ Model}

The standard k- $\varepsilon$ model is a semi-empirical model based on model transport equations for the turbulent kinetic energy $(\mathrm{k})$ and its dissipation rate $(\varepsilon)$. The model equations are derived from the Reynolds-Averaged Navier-Stokes (RANS) equations. In the derivation of the k- $\varepsilon$ model, it is assumed that the flow is fully turbulent, and the effects of molecular viscosity are negligible. The standard two-equation $k-\varepsilon$ turbulent model (ignoring any heat transfer, buoyancy effects, or external body forces) is

$$
\begin{aligned}
& \frac{\partial \rho}{\partial t}+\frac{\partial}{\partial x_{i}}\left(\rho u_{i}\right)=0 \\
& \frac{\partial}{\partial t}\left(\rho u_{i}\right)+\frac{\partial}{\partial x_{i}}\left(\rho u_{i} u_{j}\right)=-\frac{\partial p}{\partial x_{i}}+\frac{\partial \tau_{i j}}{\partial x_{j}}
\end{aligned}
$$

for a Newtonian fluid using Reynolds averaging, the stress tensor $\tau_{\mathrm{ij}}$ can be written as:

$$
\tau_{\mathrm{ij}}=\left[\mu\left(\frac{\partial \mathrm{u}_{\mathrm{i}}}{\partial \mathrm{x}_{\mathrm{j}}}+\frac{\partial \mathrm{u}_{\mathrm{j}}}{\partial \mathrm{x}_{\mathrm{i}}}\right)\right]-\frac{2}{3} \mu \frac{\partial \mathrm{u}_{\mathrm{j}}}{\partial \mathrm{x}_{\mathrm{j}}} \delta_{\mathrm{ij}}-\rho \overline{\mathrm{u}_{\mathrm{i}}^{\prime} \mathrm{u}_{\mathrm{j}}^{\prime}}
$$

The turbulent (Reynolds) stresses are modeled using the standard k- $\varepsilon$ model. The turbulent kinetic energy, $\mathrm{k}$, and its rate of dissipation, $\varepsilon$, are obtained from the following transport equations.

$$
\begin{aligned}
& \rho \frac{\mathrm{Dk}}{\mathrm{Dt}}+\frac{\partial\left(\rho \mathrm{U}_{\mathrm{i}} \mathrm{k}\right)}{\partial \mathrm{x}_{\mathrm{i}}}=\frac{\partial}{\partial \mathrm{x}_{\mathrm{j}}}\left[\left(\mu+\frac{\mu_{\mathrm{t}}}{\sigma_{\mathrm{k}}}\right) \frac{\partial \mathrm{k}}{\partial \mathrm{x}_{\mathrm{i}}}\right]+\mathrm{G}_{\mathrm{k}}-\rho \varepsilon \\
& \rho \frac{\mathrm{D} \varepsilon}{\mathrm{Dt}}+\frac{\partial\left(\rho \mathrm{U}_{\mathrm{i}} \varepsilon\right)}{\partial \mathrm{x}_{\mathrm{i}}}=\frac{\partial}{\partial \mathrm{x}_{\mathrm{j}}}\left[\left(\mu+\frac{\mu_{\mathrm{t}}}{\sigma_{\varepsilon}}\right) \frac{\partial \varepsilon}{\partial \mathrm{x}_{\mathrm{i}}}\right]+\mathrm{C}_{1 \varepsilon} \frac{\varepsilon}{\mathrm{k}} \mathrm{G}_{\mathrm{k}}-\mathrm{C}_{2 \varepsilon} \rho \frac{\varepsilon^{2}}{\mathrm{k}}
\end{aligned}
$$

The effective or turbulent viscosity, $\mu_{\mathrm{t}}$, is given by 


$$
\mu_{\mathrm{t}}=\rho \mathrm{C}_{\mu} \frac{\mathrm{k}^{2}}{\varepsilon}
$$

and $\mathrm{G}_{\mathrm{k}}$ is the production of turbulent kinetic energy,

$$
G_{k}=\mu_{t}\left(\frac{\partial u_{i}}{\partial x_{j}}+\frac{\partial u_{j}}{\partial x_{j}}\right) \frac{\partial u_{j}}{\partial x_{i}}
$$

The constants for the standard $\mathrm{k}-\varepsilon$ model are $\mathrm{C}_{1 \varepsilon}=1.44, \mathrm{C}_{2 \varepsilon}=1.92, \mathrm{C}_{\mu}=0.09, \sigma_{\mathrm{k}}=1.0$, and $\sigma_{\varepsilon}$ $=1.3$

\subsubsection{Algebraic Baldwin-Lomax Model}

The Baldwin-Lomax model is used in this work to simulate the effects of turbulence (Baldwin and Lomax, 1978). This algebraic model is easily incorporated into CFD codes and does not require the solution of any additional equations. The Baldwin-Lomax model is patterned after the Cebeci-Smith turbulence model. The Baldwin-Lomax algebraic turbulence model uses an inner and outer layer formulation.

In the inner layer, the turbulent eddy viscosity, $\mu_{t}$, is given by

$$
\left(\mu_{\mathrm{t}}\right)_{\text {inner }}=\rho(\kappa y D)^{2}|\varpi|
$$

where $\rho$ is the density, $\kappa$ is the von Karman constant, $y$ is the local distance normal to the body surface and $|\omega|$ is the magnitude of the local vorticity vector. The van Driest damping function, $\mathrm{D}$, is given by

$$
\mathrm{D}=1-\exp \left(-\frac{\mathrm{y}^{+}}{\mathrm{A}^{+}}\right)
$$

where $\mathrm{A}+$ is a constant and $\mathrm{y}+$ is given by 


$$
\mathrm{y}^{+}=\frac{\mathrm{y} \sqrt{\rho_{\mathrm{w}} \tau_{\mathrm{w}}}}{\mu_{\mathrm{w}}}
$$

where $\rho_{\mathrm{w}}$ is the density at the wall, $\mu_{\mathrm{w}}$ is the laminar viscosity at the wall and $\tau_{\mathrm{w}}$ is the wall shear stress. In the outer layer, the turbulent eddy viscosity is given by

$$
\left(\mu_{\mathrm{t}}\right)_{\text {outer }}=\mathrm{KC}_{\mathrm{cp}} \rho \mathrm{F}_{\mathrm{WAKE}} \mathrm{F}_{\mathrm{kelb}}(\mathrm{y})
$$

where $\mathrm{K}$ is the Clauser constant and $\mathrm{C}_{\mathrm{cp}}$ is an additional constant. For wall bounded shear flows, $\mathrm{F}_{\text {wake }}$ is given by

$$
\mathrm{F}_{\text {wake }}=\mathrm{y}_{\max } \mathrm{F}_{\max }
$$

where $y_{\max }$ and $F_{\max }$ correspond to the location of the maximum value of the "vorticity" function,

$$
\mathrm{F}(\mathrm{y})=\mathrm{y}|\varpi| \mathrm{D}
$$

The Klebanoff intermittency factor is given by

$$
\mathrm{F}_{\text {Kleb }}(\mathrm{y})=1 /\left[1+5.5\left(\frac{\mathrm{C}_{\text {kleb }} \mathrm{y}}{\mathrm{y}_{\max }}\right)^{6}\right]
$$

where $C_{K l e b}$ is a constant. The constants in the model have the following values $A^{+}=26, C_{c p}$ $=1.6, \mathrm{C}_{\mathrm{Kleb}}=0.3, \kappa=0.4, \mathrm{~K}=0.0180$. 
The turbulent eddy viscosity becomes

$$
\mu_{\mathrm{t}}=\left\{\begin{array}{l}
\left(\mu_{\mathrm{t}}\right)_{\text {inner }}, \mathrm{y}<=\mathrm{y}_{\text {cross }} \\
\left(\mu_{\mathrm{t}}\right)_{\text {outer }}, \mathrm{y}>=\mathrm{y}_{\text {cross }}
\end{array}\right\}
$$

where $y_{\text {cross }}$ is the smallest value of $y$ at which the inner layer and outer layer formulations for the turbulent eddy viscosity are equal.

\subsection{Models for Multiphase Flows}

\subsubsection{Multiphase CFD}

Numerical calculations have been made of particle dispersion and particle-fluid interactions in turbulent flows. For engineering problems, two approaches based on the Reynolds-averaged Navier-Stokes equations are commonly applied, namely the Euler-Euler approach and the Euler-Lagrange method.

\subsubsection{Euler-Euler Approach}

In the Euler-Euler approach both solid and liquid phases are considered as an interacting continua. The consideration of a particle size and density distribution or other important material properties in HSBS requires the solution of a set of basic equations for each class to be considered. Hence the computational effort increases with the number of size classes or density fractions rapidly. The method is however preferable for discontinuous and dense twophase flows, as, for example, found in fluidized-beds of uniform particles. A detailed review on recent development of Eulerian models for the predication of fluidization processes was recently published by Enwald et al (1996). Thus, this approach could be used in cases when 
dense solid or gas two phase flows exists where all parameters are defined and used as average variables, while ignoring the discrete characteristics of dispersed phase.

\subsubsection{Euler-Lagrange Approach}

The second approach, the Euler-Lagrange approach, involving calculating the fluid flow in an Euler frame with the particles being moved individually in Lagrangian coordinates. The Euler-Lagrange approach is applicable to problems of the dispersed phases and accounts for the discrete nature of the individual particles. Generally, the particles are considered as point particles, i.e. the finite dimension of the particles is not considered and the flow around the individual particles is not resolved, since the number of real particles in a flow system is usually too large to permit tracking of all particles. The trajectories of computational particles (i.e. parcels) which represent a number of real particles with the same properties (i. e. size, velocity and temperature) are calculated. Although the high requirement of computational capability arises from application of Lagrangian approach, some techniques are developed to reduce this computational cost while maintaining equivalent accuracy of final calculation results. Based on previous work by Oesterle \& Petitjean (1993) and Sommerfield \& Zivkovic (1992) a stochastic inter-particle collision model was developed, which also accounts for the velocity correlation of colliding particles and is much more efficient (Sommerfeld, 1995 and 1999). An implicit particle interaction mechanism is proposed by Smirnov and Celik (2000). These computational techniques do not require keeping track of the neighboring particles and completely avoid expensive looping over the neighbor-particles subsets and reduces the computation requirements for particle collisions. 


\subsubsection{Selection of Multiple Phase Models}

The Euler-Euler model on the other hand treats the dispersed phase as a quasi-continuum, assuming that each element finite volume of the space domain contains a respective fraction of the continuous and of the dispersed phase. To be physically meaningful, the minimum size of the space volume element must be substantially larger than a single dispersed particle. In the case of particular flow, the equations derived for Euler-Euler models consist of the Navier-Stokes and mass continuity equations for the solid phase and the liquid phase. However, the collision and re-dispersion of particles can not be explicitly considered in this model.

In the Euler-Lagrange model, one quasi-homogeneous gas-liquid phase is considered within which the flow of the dispersed phase particles is tracked. That means for a particular flow, the traces of all individual particle or bubbles in the moving continuous phase have to be obtained through solving the respective equations of motion.

An important and obvious advantage of the Euler-Lagrangian formulation comes from the fact that each individual particle is modeled in its flow through the column. This allows for a direct consideration of additional effects related to inter-particle and particle-liquid interaction. Mass transfer without and with chemical reaction, particle collisions and particlewall collisions can, in principle, be added directly to an Euler-Lagrange hydrodynamic model. A second advantage lies in the fact that no numerical diffusion will be introduced into the dispersed phase since the trajectory of each particle can be calculated accurately within a given volume element.

In the hindered-settling process, the feed coal usually has a wide size distribution and complicated density composition. Therefore, it is obvious that the Euler-Euler formulation is 
not the right choice for this application. In this study, the Euler-Lagrange approach was extended and applied to solid-liquid flow simulation in HSBS.

\subsection{Fundamentals of Euler-Lagrange Approach}

The Euler-Lagrange approach was extended into time-dependent calculation of solidliquid in a hindered-settling separator by solving the Reynolds-averaged Navier-Stokes equations together with the Baldwin-Lomax turbulent model through which the particular induced turbulence is ignored. Movements of particles are considered by accounting for all relevant forces. The coupling effect of solid particles on the liquid phase is described through a turbulent model modification. The moment transfer between particles is calculated using collision models. The liquid phase is considered as a continuous phase, and the solid phase is accounted as a dispersed phase.

\subsubsection{Liquid Phase Hydrodynamics}

The liquid phase is calculated by solving the unsteady Reynolds-averaged conservation equations using a well-known Baldwin-Lomax turbulence model (Baldwin and Lomax, 1978).

The continuous equation without mass transfer and chemical reactions becomes:

$$
\frac{\partial}{\partial t}(\rho \phi)+\frac{\partial}{\partial x_{i}}\left(\rho \phi U_{i}\right)=0
$$

The momentum balance for the continuous phase in multiphase flow is described in the following general formulation:

$$
\begin{gathered}
\frac{\partial}{\partial t}\left(\rho \phi U_{i}\right)+\frac{\partial}{\partial x_{i}}\left(\rho \phi U_{i} U_{j}\right)=-\frac{\partial P}{\partial x_{i}}+\frac{\partial}{\partial x_{j}} \Gamma\left(\frac{\partial U_{i}}{\partial x_{j}}+\frac{\partial U_{j}}{\partial x_{i}}\right)+\rho \phi g_{i}+F_{w i} \\
\Gamma=\mu+\mu_{t}
\end{gathered}
$$


where $\phi$ is the liquid phase volume fraction in a computation cell.

The above differential equations expressing conservation of mass and momentum for liquid phase in two dimensions can also be written in Cartesian coordinate system by substituting $\mathrm{x}, \mathrm{y}$ for $\mathrm{i}, \mathrm{j}$ :

$$
\begin{gathered}
\frac{\partial}{\partial \mathrm{t}}(\rho \phi)+\frac{\partial}{\partial \mathrm{x}}(\rho \phi \mathrm{U})+\frac{\partial}{\partial \mathrm{y}}(\rho \phi \mathrm{V})=0 \\
\frac{\partial}{\partial \mathrm{t}}(\rho \phi \mathrm{U})+\phi \rho\left(\mathrm{u} \frac{\partial \mathrm{u}}{\partial \mathrm{x}}+\mathrm{v} \frac{\partial \mathrm{u}}{\partial \mathrm{y}}\right)=-\frac{\partial \mathrm{p}}{\partial \mathrm{x}}+\rho \mathrm{g}_{\mathrm{x}}+\frac{\partial}{\partial \mathrm{x}}\left(\Gamma \frac{\partial \mathrm{u}}{\partial \mathrm{x}}\right)+\frac{\partial}{\partial \mathrm{y}}\left(\Gamma \frac{\partial \mathrm{u}}{\partial \mathrm{y}}\right)+\mathrm{F}_{\mathrm{wx}} \\
\frac{\partial}{\partial \mathrm{t}}(\rho \varphi \mathrm{V})+\varphi \rho\left(\mathrm{u} \frac{\partial \mathrm{v}}{\partial \mathrm{x}}+\mathrm{v} \frac{\partial \mathrm{v}}{\partial \mathrm{y}}\right)=-\frac{\partial \mathrm{p}}{\partial \mathrm{y}}+\rho \mathrm{g}_{\mathrm{y}}+\frac{\partial}{\partial \mathrm{x}}\left(\Gamma \frac{\partial \mathrm{v}}{\partial \mathrm{x}}\right)+\frac{\partial}{\partial \mathrm{y}}\left(\Gamma \frac{\partial \mathrm{v}}{\partial \mathrm{y}}\right)+\mathrm{F}_{\mathrm{wy}}
\end{gathered}
$$

The Baldwin-Lomax model is one of the most wide-spread and accepted turbulence models, and it is easy to incorporate into computer program. It has been chosen in this study to calculate the turbulent viscosity for liquid phase.

\subsubsection{Particle Movement}

The movement of particles involves linear motion and particle rotation. The calculation of particle locations, the linear and angular velocities require the integrations of the following three ordinary differential equations:

$$
\begin{aligned}
& \frac{d \vec{X}_{p}}{d t}=\vec{U}_{p} \\
& m_{p} \frac{d \vec{U}_{p}}{d t}=F_{\text {total }} \\
& I_{p} \frac{d \vec{\omega}_{p}}{d t}=\vec{T}
\end{aligned}
$$


where $U_{p}$ is instant velocity of particle, $X_{p}$ is the coordinate of particle, $\omega_{p}$ is particle angular velocity $\mathrm{I}_{\mathrm{p}}=\left(0.1 \mathrm{~m}_{\mathrm{p}} \mathrm{D}_{\mathrm{p}}^{2}\right)$ is the moment of inertia for a spherical particle, $\overrightarrow{\mathrm{F}}_{\text {total }}$ represents all the relevant applicable linear forces acting on the particle in the fluid, and $\vec{T}$ is the torque acting on a rotating particle by the local fluid. The instantaneous fluid velocity components at the particle location required for calculation of forces in Eq. (3-27) are determined from the local mean fluid velocity interpolated from the neighboring Euler grid points using area weighted averaging techniques.

It is assumed that the forces $\vec{F}_{\text {total }}$ acting on a solid spherical particle moving in an unsteady and non-uniform liquid flow field is composed of separate and uncoupled contributions from the fluid drag force, the pressure gradient force, the added mass force, the Mangus force (Slip-Rotation Lift force), the Saffman force (Slip-Shear Lift force) and the gravitational body force. A particle moving relative to a liquid accelerates part of fluid around it. This slip velocity leads to an unbalanced pressure distribution as well as viscous stresses on the particle surface. This yields a resulting force known as the drag or friction force. The drag force is usually the dominant force contribution to the particle movement if the size of the particle is small enough. The drag force takes into account the interaction forces between the liquid and the particles in a uniform flow field under non-accelerating conditions. However, if the particle is accelerated relative to the liquid, a portion of the surrounding liquid has to accelerate as well. This additional force contribution is called the added mass force. In a region where a velocity gradient exists, for instance, near a wall or in a high shear region, a particle moving at a constant velocity is subjected to a lift force caused by the velocity gradient. This force is also called the Saffman force. Particle rotation may result from particle-particle collisions or wall-particle collisions, or from a velocity gradient 
Table 3.1 Forces acting on a particle settling in an unsteady and non-uniform liquid flow in hindered-settling separator

\begin{tabular}{|c|c|c|}
\hline Force & Expression & \\
\hline Drag force & $\begin{array}{c}\overrightarrow{\mathrm{F}}_{\mathrm{D}}=\frac{3}{4} \frac{\rho_{\mathrm{F}} \mathrm{m}_{\mathrm{P}}}{\rho_{\mathrm{P}} \mathrm{D}_{\mathrm{P}}} \mathrm{C}_{\mathrm{D}}\left(\overrightarrow{\mathrm{U}}_{\mathrm{F}}-\overrightarrow{\mathrm{U}}_{\mathrm{P}}\right)\left|\overrightarrow{\mathrm{U}}_{\mathrm{F}}-\overrightarrow{\mathrm{U}}_{\mathrm{P}}\right| \\
\mathrm{C}_{\mathrm{D}}=\left\{\begin{array}{c}24 \operatorname{Re}_{\mathrm{P}}^{-1}\left(1+0.15 \operatorname{Re}_{\mathrm{P}}^{0.687}\right) \\
0.44\end{array}\right. \\
\operatorname{Re}_{\mathrm{P}}=\frac{\rho_{\mathrm{F}} \mathrm{D}_{\mathrm{P}}\left|\overrightarrow{\mathrm{U}}_{\mathrm{F}}-\overrightarrow{\mathrm{U}}_{\mathrm{P}}\right|}{\mu_{\mathrm{F}}}\end{array}$ & $\begin{array}{l}\operatorname{Re}_{\mathrm{P}}<1000 \\
\operatorname{Re}_{\mathrm{P}}>1000\end{array}$ \\
\hline $\begin{array}{l}\text { Pressure gradient } \\
\text { force }\end{array}$ & $\overrightarrow{\mathrm{F}}_{\mathrm{P}}=-\frac{\mathrm{m}_{\mathrm{P}}}{\rho_{\mathrm{P}}} \nabla \mathrm{P}, \quad \nabla \mathrm{P}=\rho_{\mathrm{F}} \frac{\mathrm{D \textrm {U } _ { \mathrm { F } }}}{\mathrm{Dt}}$ & \\
\hline Added mass force & $\mathrm{F}_{\mathrm{AD}}=0.5 \mathrm{~m}_{\mathrm{F}}\left(\frac{\mathrm{D} \overrightarrow{\mathrm{U}}}{\mathrm{Dt}}-\frac{\mathrm{D} \overrightarrow{\mathrm{U}_{\mathrm{P}}}}{\mathrm{Dt}}\right)$ & \\
\hline Mangus force & $\begin{array}{l}\overrightarrow{\mathrm{F}}_{\mathrm{M}}=\frac{\rho_{\mathrm{F}}}{2} \frac{\pi}{4} \mathrm{D}_{\mathrm{P}}^{2} \mathrm{C}_{\mathrm{LR}}\left|\overrightarrow{\mathrm{U}}_{\mathrm{F}}-\overrightarrow{\mathrm{U}}_{\mathrm{P}}\right| \frac{\vec{\Omega} \times\left(\overrightarrow{\mathrm{U}}_{\mathrm{F}}-\overrightarrow{\mathrm{U}}_{\mathrm{P}}\right)}{|\vec{\Omega}|} \\
\vec{\Omega}_{\mathrm{F}}=\frac{1}{2} \nabla \times \overrightarrow{\mathrm{U}}_{\mathrm{F}}-\vec{\omega}_{\mathrm{P}} \\
\mathrm{C}_{\mathrm{LR}}=\frac{\operatorname{Re}_{\mathrm{R}}}{\operatorname{Re}_{\mathrm{P}}} \quad \operatorname{Re}_{\mathrm{R}}=\frac{\rho_{\mathrm{F}} \mathrm{D}_{\mathrm{P}}^{2}|\vec{\Omega}|}{\mu_{\mathrm{F}}}\end{array}$ & \\
\hline Saffman force & $\begin{array}{l}\overrightarrow{\mathrm{F}}_{\mathrm{S}}=\frac{\rho_{\mathrm{F}}}{2} \frac{\pi}{4} \mathrm{D}_{\mathrm{P}}^{3} \mathrm{C}_{\mathrm{LS}}\left(\left(\overrightarrow{\mathrm{U}}_{\mathrm{F}}-\overrightarrow{\mathrm{U}}_{\mathrm{P}}\right) \times \vec{\omega}_{\mathrm{F}}\right) \\
\vec{\omega}_{\mathrm{F}}=\nabla \times \overrightarrow{\mathrm{U}}_{\mathrm{F}}, \quad \mathrm{C}_{\mathrm{LS}}=0.53\end{array}$ & \\
\hline Body force & $\overrightarrow{\mathrm{F}}_{\mathrm{B}}=\left(\mathrm{m}_{\mathrm{p}}-\mathrm{m}_{\mathrm{F}}\right) \overrightarrow{\mathrm{g}}$ & \\
\hline
\end{tabular}


in a non-uniform flow region. The particle rotation causes the fluid velocity difference around particle, which yields an unbalanced pressure distribution around the particle. Thus, a lift force is established which moves the particle to a region of higher velocity. This lift force is also called the Magnus force.

The detailed definitions of the different forces are given in Table 3.1. The summation of forces acting on particles is defined as:

$$
\overrightarrow{\mathrm{F}}_{\text {total }}=\overrightarrow{\mathrm{F}}_{\mathrm{D}}+\overrightarrow{\mathrm{F}}_{\mathrm{P}}+\overrightarrow{\mathrm{F}}_{\mathrm{AD}}+\overrightarrow{\mathrm{F}}_{\mathrm{M}}+\overrightarrow{\mathrm{F}}_{\mathrm{S}}+\overrightarrow{\mathrm{F}}_{\mathrm{B}}
$$

where, $\mathrm{m}_{\mathrm{ap}}=\pi / 6 \rho_{\mathrm{P}} \mathrm{D}_{\mathrm{P}}{ }^{3}$ is the particle mass, $\mathrm{m}_{\mathrm{F}}=\pi / 6 \rho \mathrm{D}_{\mathrm{P}}{ }^{3}$ is mass of a sphere of liquid with a diameter of $D_{P}, \quad \nabla P=\rho_{F} \frac{D \vec{U}_{F}}{D t}$ is the pressure gradient related to fluid, acceleration, $\vec{\omega}_{F}=\nabla \times \vec{U}_{F}$ is the fluid rotation angle velocity, $\vec{\Omega}_{F}=\frac{1}{2} \nabla \times \vec{U}_{F}-\vec{\omega}_{P}$ is the relative rotation between liquid and particle, $C_{D}$ is drag coefficient, $C_{L S}$ (Delong et al., 1997) is the shear lift coefficient, $\mathrm{C}_{\mathrm{LR}}$ (Rubio and Keller, 1961) is the rotation lift coefficient.

\subsubsection{Coupling between the Phases}

The particles occupy the computational cell and reduce the liquid volume fraction. They also exert interaction forces on the surrounding liquid phase. Thus, the two phases are coupled through the liquid volume fraction and through the total source force term that accounts for the momentum transfer from the particles to the liquid. Both the liquid volume fraction and the total source term can be calculated from the number of particles in a computational cell. 


\subsubsection{Liquid Volume Fraction}

The liquid volume fraction, $\phi$, in a computational cell is calculated by reducing total volumes of all dispersed $\mathrm{n}$ number of particles inside a computation cell from a computational cell volume (Figure 3.1):

$$
\phi=1-\frac{\sum_{i=1}^{n} V_{P i}}{V_{\text {cell }}}
$$

where $\mathrm{V}_{\text {IPs }}$ is the volume of particle $\mathrm{i}, \mathrm{V}_{\text {cell }}$ is the volume of the computation cell.

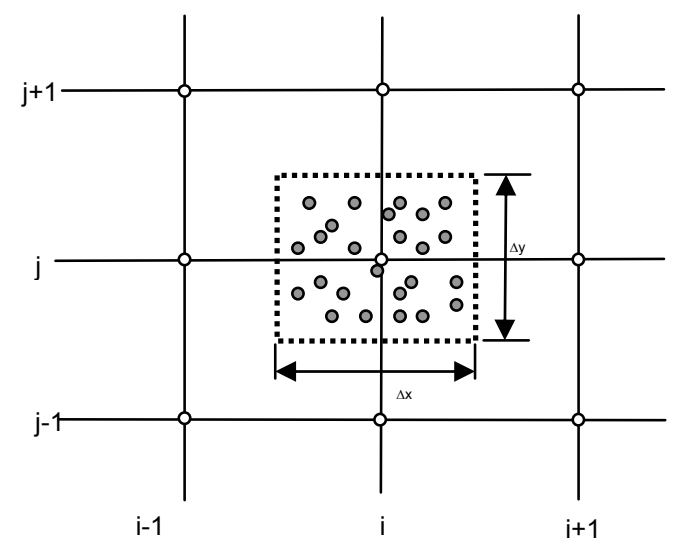

• = particle; $\mathrm{o}=$ gird node; $\mathrm{i}, \mathrm{j}=$ index of grid node;

Figure 3.1 Calculation of liquid volume fraction in a computational cell

\subsubsection{Momentum Transfer Term}

The particles in a HSBS are driven upward by fluidizing water flow through acting forces from the liquid phase and the body force. On the other hand, the surrounding liquid flow of particles is exposed by the opposite forces exerted to the liquid on the particles. 
The momentum transfer terms in Eqs. (3-24) and (3-25) describe the interaction forces between the liquid phase and dispersed phase per unit computational cell. Only pressure and gravity forces are acting on a motionless particle in a motionless liquid. The momentum transfer term describes the interaction between the liquid phase and the solid phase when there is relative slip velocity and angular velocity between the particles and the surrounding liquid. Thus, the pressure gradient force and the gravity force can not be included in the momentum transfer term back to the fluid. In this study, four different contributions for the interaction force term are taken into account, the drag force term $\overrightarrow{\mathrm{F}}_{\mathrm{D}}$, the added mass force term $\overrightarrow{F_{A D}}$, the Mangus force term $\vec{F}_{M}$, and the Saffman force term $\vec{F}_{M}$. For a single particle, $\mathrm{i}$, the corresponding source force term of this particle is given as:

$$
\overrightarrow{\mathrm{F}}_{\mathrm{Wi}}=\overrightarrow{\mathrm{F}}_{\mathrm{Di}}+\overrightarrow{\mathrm{F}}_{\mathrm{ADi}}+\overrightarrow{\mathrm{F}}_{\mathrm{Mi}}+\overrightarrow{\mathrm{F}}_{\mathrm{Si}}
$$

The opposite sign of the force term in Eq. (3-31) is used when substituting back into Eqs. (324) and (3-25) because they are opposing forces exerted back by the particles on the liquid phase.

Due to the discrete dispersion nature of particles in a computational cell, the effect of the source term $\left(\mathrm{Fr}_{\mathrm{i}}\right)$ of a particle in a fluid computation cell is calculated through area-weighted averaging techniques shown in Figure 3.2. 


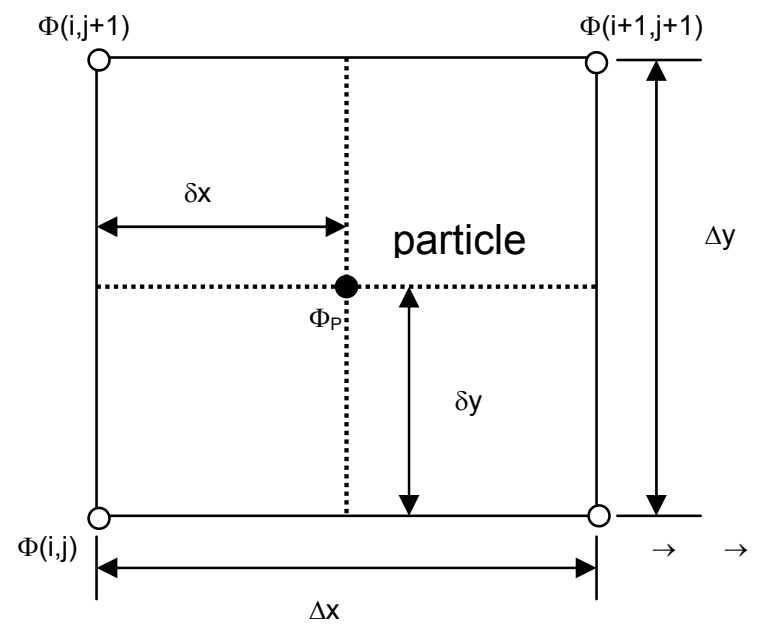

- = particle; $\mathrm{o}=$ grid node; $\Phi=$ variables; $\mathrm{i}, \mathrm{j}=$ index of node

Figure 3.2 Area-weighted averaging of variables over a computation cell

$$
\begin{aligned}
& \Phi_{\mathrm{i}, \mathrm{j}}=\frac{(\Delta \mathrm{x}-\delta \mathrm{x})(\Delta \mathrm{y}-\delta \mathrm{y})}{\Delta \mathrm{x} \Delta \mathrm{y}} \Phi_{\mathrm{P}} \\
& \Phi_{\mathrm{i}+1, \mathrm{j}}=\frac{\delta \mathrm{x}(\Delta \mathrm{y}-\delta \mathrm{y})}{\Delta \mathrm{x} \Delta \mathrm{y}} \Phi_{\mathrm{P}} \\
& \Phi_{\mathrm{i}+1, \mathrm{j}+1}=\frac{\delta \mathrm{x} \delta \mathrm{y}}{\Delta \mathrm{x} \Delta \mathrm{y}} \Phi_{\mathrm{P}} \\
& \Phi_{\mathrm{i}, \mathrm{j}+1}=\frac{(\Delta \mathrm{x}-\delta \mathrm{x}) \delta \mathrm{y}}{\Delta \mathrm{x} \Delta \mathrm{y}} \Phi_{\mathrm{P}}
\end{aligned}
$$

If there are $n$ particles in a computation cell which is centered at grid node point $(i, j)$, then the total value of variable $\Phi_{\mathrm{pk}}$ from discrete particles on grid node point $(\mathrm{i}, \mathrm{j})$ is given as:

$$
\Phi_{\text {total(i,j) }}=\sum_{\mathrm{k}=1}^{\mathrm{n}} \Phi_{\mathrm{p}_{\mathrm{k}}}
$$

For the calculation of particle motion, the local values for pressure, the linear and angular velocities components, and the liquid viscosity at the location of the particle are required. 
These local values can also be calculated by an area-weighted averaging approach. As shown in Figure 3.2, if the value of a variable, $\Phi$, at each grid node is known, then the local value of a variable, $\Phi_{\mathrm{p}}$ at location of particle, $\mathrm{p}$, can be calculated by $\Phi_{\mathrm{p}}=\frac{1}{\Delta \mathrm{x} \Delta \mathrm{y}}\left[(\Delta \mathrm{x}-\delta \mathrm{x})(\Delta \mathrm{y}-\delta \mathrm{y}) \Phi_{\mathrm{i}, \mathrm{j}}+(\Delta \mathrm{x}-\delta \mathrm{x}) \delta \mathrm{y} \Phi_{\mathrm{i}, \mathrm{j}+1}+\delta \mathrm{x}(\Delta \mathrm{y}-\delta \mathrm{y}) \Phi_{\mathrm{i}, \mathrm{j}}+\delta \mathrm{x} \delta \mathrm{y} \Phi_{\mathrm{i}+1, \mathrm{j}+1}\right]$ 


\section{Chapter 4 Particle Movement and Particle Collisions}

After the liquid velocity profile is known, the motion of particles in the liquid flow can be described in a Lagrangian frame by solving a set of ordinary differential equations along the trajectory to obtain a new object location, as well as the linear and angular components of the particle velocity. This requires the consideration of all relevant forces acting on the particles in liquid at first. In this chapter, the details of definition and derivation for different forces are described.

\subsection{Forces on Particles in Fluids}

\subsubsection{Drag Force}

Drag force is defined as

$$
\vec{F}_{D}=\frac{3}{4} \frac{\rho_{F} M_{P}}{\rho_{P} D_{P}} C_{D}\left(\vec{U}_{F}-\vec{U}_{P}\right)\left|\vec{U}_{F}-\vec{U}_{P}\right|
$$

In most fluid-particle systems the drag force is the dominant force that affects the particle motion. The derivation of the drag force at higher particle Reynolds numbers is based on a drag coefficient $C_{D}$ which is defined as:

$$
C_{D}=\frac{F_{D}}{\frac{\rho_{F}}{2}\left(\vec{U}_{F}-\vec{U}_{P}\right)^{2} A_{P}}
$$


where $\mathrm{A}_{\mathrm{p}}=\pi / 4 \mathrm{D}_{\mathrm{p}}{ }^{2}$ is the cross-section of a spherical particle. It is well known that the drag coefficient is a function of the particle's Reynolds number.

$$
\mathrm{Re}_{\mathrm{p}}=\frac{\rho_{\mathrm{F}} \mathrm{D}_{\mathrm{p}}\left(\overrightarrow{\mathrm{U}}_{\mathrm{F}}-\overrightarrow{\mathrm{U}}_{\mathrm{P}}\right)}{\mu_{\mathrm{F}}}
$$

The dependence of the drag coefficient of a sphere as shown in Figure 4.1 is based on numerous experimental investigations (Schlichting 1965). Several regimes which are associated with flow characteristics around the sphere depending on Reynolds numbers are listed below:

For small Reynolds numbers (i.e. Rep $<0.5$ ), viscous effects are dominating and no flow separation is observed, the drag coefficient is proposed as

$$
C_{D}=\frac{24}{\operatorname{Re}_{p}}
$$

This regime is often referred to as the Stokes-regime.

In the transition region (i.e. $0.5<$ Rep $<1000$ ) inertial effects become of increasing importance. Above a Reynolds number of about 24 the flow around the particle begins to separate. Initially this separation is symmetric (Clift et al. 1978). It becomes unstable and periodic above Rep $\approx 130$. For this non-linear regime numerous correlations have been proposed (Clift et al. 1978, Crowe et al. 1998) which fit the experimental data more or less accurately. A frequently referenced correlation is that proposed by Schiller \& Naumann (1933), which fits the data up to $\mathrm{Re}_{\mathrm{p}}=1000$ reasonably well (see Figure 4.1).

$$
C_{D}=\frac{24}{\operatorname{Re}_{p}}\left(1+0.15 \operatorname{Re}_{\mathrm{p}}^{0.687}\right)=\frac{24}{\operatorname{Re}_{\mathrm{p}}} \mathrm{f}_{\mathrm{D}}
$$


Above $\mathrm{Re}_{\mathrm{p}} \approx 1000$ the drag coefficient remains almost constant up to the critical Reynolds number, since the wake size and structure is not changing considerably. This regime is referred to as Newton-regime with:

$$
\mathrm{C}_{\mathrm{D}} \approx 0.44
$$

At the critical Reynolds number $\left(\operatorname{Re}_{\text {crit }} \approx 2.5 \times 10^{5}\right)$ a drastic decrease of the drag coefficient is observed. This is caused by the transition from a laminar to a turbulent boundary layer around the particle. This results in a decrease of the particle wake.

In the super-critical region (i.e. Rep $>4.0 \times 10^{5}$ ) the drag coefficient again increases continuously. For most practical particulate flows however this region is not relevant.

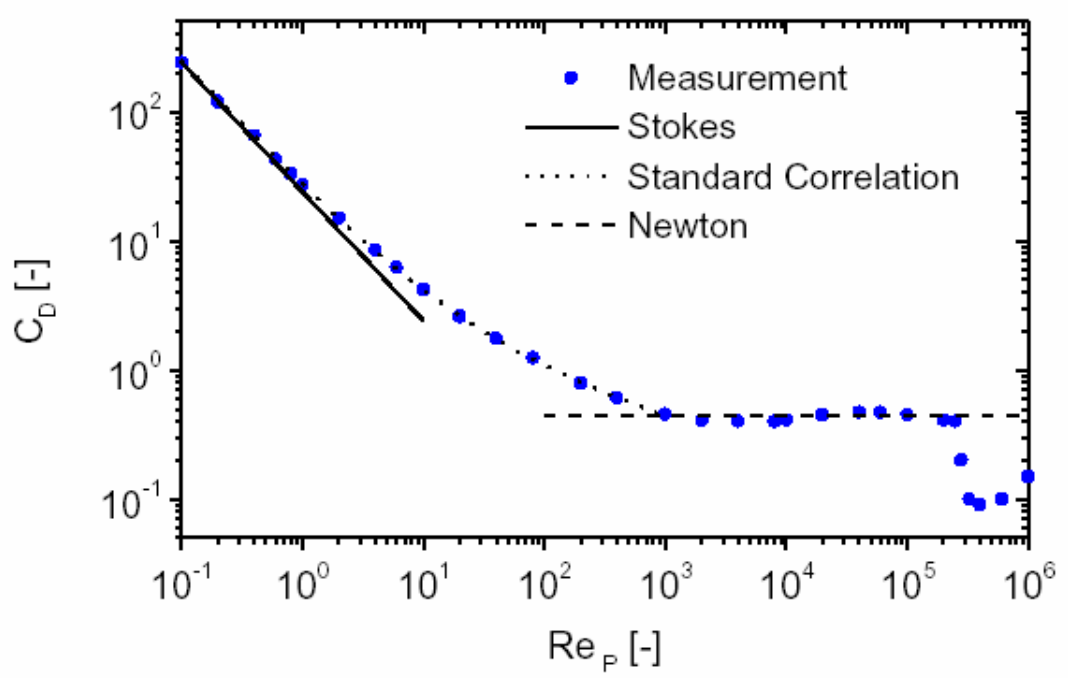

Figure 4.1 Drag coefficients as a function of particle Reynolds number for the different regimes (Source: Schlichting, 1965) 


\subsubsection{Pressure Gradient and Buoyancy Force}

The pressure force from the pressure gradient and shear stress is defined as:

$$
\mathrm{F}_{\mathrm{P}}=\frac{\mathrm{m}_{\mathrm{p}}}{\rho_{\mathrm{p}}}(-\nabla \mathrm{p}+\nabla \vec{\tau})
$$

From the Navier-Stokes equation of the fluid the pressure gradient and the shear stress can be related to the fluid acceleration and the gravity force:

$$
-\nabla \mathrm{p}+\nabla \vec{\tau}=\rho_{\mathrm{F}}\left(\frac{\mathrm{D} \vec{U}_{\mathrm{F}}}{\mathrm{Dt}}-\overrightarrow{\mathrm{g}}\right)
$$

Hence, the total pressure force is obtained in the following form:

$$
\vec{F}_{p}=m_{p} \frac{\rho_{F}}{\rho_{P}}\left(\frac{D \vec{U}_{F}}{D t}-\vec{g}\right)
$$

The first term of Eq. (4-9) represents the fluid acceleration and the second one is the buoyancy force.

\subsubsection{Added Mass Force}

The acceleration or deceleration of a particle in a fluid also requires the acceleration or deceleration of a certain fraction of the surrounding fluid. This is the so-called added mass. Analytic solutions for added mass forces are only possible for small particle Reynolds numbers. An extension to higher particle Reynolds numbers is only possible by introducing empirical coefficients similar to the drag coefficient for all the forces.

Based on an experimental study by Odar and Hamilton (1964) who studied the motion of a sphere in fluid, the added mass is expressed as

$$
\mathrm{F}_{\mathrm{A}}=0.5 \mathrm{C}_{\mathrm{A}} \rho_{\mathrm{F}} \frac{\mathrm{m}_{\mathrm{p}}}{\rho_{\mathrm{p}}} \frac{\mathrm{d}}{\mathrm{dt}}\left(\overrightarrow{\mathrm{U}}_{\mathrm{F}}-\overrightarrow{\mathrm{U}}_{\mathrm{P}}\right)
$$


Where, coefficient 0.5 is the accelerated portion of the fluid volume ( 0.5 for spheres and 1.0 for cylinders) placed transverse to the flow (Schulze, 1984). The coefficients $C_{A}$ was obtained from the experiments of Odar and Hamilton (1964) in the following form:

$$
\mathrm{C}_{\mathrm{A}}=2.1-\frac{0.132}{\mathrm{~A}_{\mathrm{C}}^{2}+0.12}
$$

The parameter $A_{C}$ is called the acceleration number and is defined by

$$
\mathrm{A}_{\mathrm{C}}=\frac{\left|\overrightarrow{\mathrm{U}}_{\mathrm{F}}-\overrightarrow{\mathrm{U}}_{\mathrm{P}}\right|}{\mathrm{D}_{\mathrm{P}}\left|\frac{\mathrm{d} \overrightarrow{\mathrm{U}}_{\mathrm{F}}-\overrightarrow{\mathrm{U}}_{\mathrm{P}} \mid}{\mathrm{dt}}\right|}
$$

\subsubsection{Body Forces}

Body forces are the gravity forces, or other forces that are acting on particles when the particle moves in gravitational, electric or magnetic field. The gravity force is

$$
\overrightarrow{\mathrm{F}_{\mathrm{g}}}=\mathrm{m}_{\mathrm{p}} \overrightarrow{\mathrm{g}}
$$

It is should be noted that this force is already included in pressure force if only the gravity field is applicable.

\subsubsection{Slip-Shear Lift Force (Saffman Force)}

A moving particle in a shear layer experiences a transverse lift force due to the nonuniform relative velocity over the particle and the resulting non-uniform pressure distribution. The lift force is acting towards the direction of high slip velocity. An expression for the slip shear force for a freely rotating particle moving at constant velocity in a two- 
dimensional shear flow at low Reynolds number was derived from an asymptotic expansion by Saffman (1965).

$$
\mathrm{F}_{\mathrm{LS}, \text { Saff }}^{\mathrm{Y}}=6.46 \frac{\mathrm{D}_{\mathrm{P}}^{2}}{4}\left(\rho_{\mathrm{F}} \mu_{\mathrm{F}}\right)^{0.5}\left|\frac{\partial \mathrm{U}_{\mathrm{F}}}{\partial \mathrm{y}}\right|\left(\mathrm{U}_{\mathrm{F}}-\mathrm{U}_{\mathrm{P}}\right)
$$

Expanding this expression to a three dimensional flow and introducing a correction function for a higher particle Reynolds numbers yields

$$
\overrightarrow{\mathrm{F}}_{\mathrm{LS}}=1.615 \mathrm{D}_{\mathrm{P}}^{2}\left(\rho_{\mathrm{F}} \mu_{\mathrm{F}}\right)^{0.5}\left(\frac{1}{\left|\overrightarrow{\omega_{\mathrm{F}}}\right|}\right)^{0.5}\left\{\left(\overrightarrow{\mathrm{U}}_{\mathrm{F}}-\overrightarrow{\mathrm{U}}_{\mathrm{P}}\right) \times \vec{\omega}_{\mathrm{F}}\right\} \mathrm{f}\left(\operatorname{Re}_{\mathrm{P}}, \operatorname{Re}_{\mathrm{S}}\right)
$$

Here the fluid rotation is obtained from

$$
\vec{\omega}_{\mathrm{F}}=\nabla \times \overrightarrow{\mathrm{U}}_{\mathrm{F}}
$$

Introducing now a lift coefficient in Eq. (4-15) gives the following expression for the slipshear lift force:

$$
\overrightarrow{\mathrm{F}}_{\mathrm{LS}}=\frac{\rho_{\mathrm{F}}}{2} \frac{\pi}{4} \mathrm{D}_{\mathrm{P}}^{2} \mathrm{C}_{\mathrm{LS}} \mathrm{D}_{\mathrm{P}}\left\{\left(\overrightarrow{\mathrm{U}}_{\mathrm{F}}-\overrightarrow{\mathrm{U}}_{\mathrm{P}}\right) \times \vec{\omega}_{\mathrm{F}}\right\}
$$

The lift coefficient is

$$
C_{\text {LS }}=\frac{4.1126}{\operatorname{Re}_{\mathrm{S}}^{0.5}} \mathrm{f}\left(\operatorname{Re}_{\mathrm{P}}, \operatorname{Re}_{\mathrm{s}}\right)
$$

The correction function $\mathrm{f}\left(\mathrm{Re}_{\mathrm{p}}, \mathrm{Re}_{\mathrm{s}}\right)$ proposed by Mei (1992) based on calculations performed by Dandy and Dwyer (1990) for a particle Reynolds number in the range $0.1 \leq$ Rep $\leq 100$ is given by

$$
\mathrm{f}\left(\operatorname{Re}_{\mathrm{P}}, \operatorname{Re}_{\mathrm{s}}\right)=\frac{\mathrm{F}_{\mathrm{LS}}}{\mathrm{F}_{\mathrm{LS}, \text { Saff }}}
$$


where

$$
\begin{aligned}
\frac{F_{L S}}{F_{L S, S a f f}} & =\left(1-0.3314 \beta^{0.5}\right) \exp \left(-\frac{\operatorname{Re}_{\mathrm{P}}}{10}\right)+0.3314 \beta^{0.5} & & \text { for } \operatorname{Re}_{\mathrm{p}} \leq 40 \\
& =0.0524 \quad\left(\beta \operatorname{Re}_{\mathrm{P}}\right)^{0.5} & & \text { for } \operatorname{Re}_{\mathrm{P}}>40
\end{aligned}
$$

with

$$
\beta=0.5 \frac{\operatorname{Re}_{\mathrm{S}}}{\operatorname{Re}_{\mathrm{P}}}
$$

and the Reynolds number of the shear flow.

$$
\operatorname{Re}_{\mathrm{S}}=\frac{\rho_{\mathrm{F}} \mathrm{D}_{\mathrm{P}}^{2}\left|\vec{\omega}_{\mathrm{F}}\right|}{\mu_{\mathrm{F}}}
$$

\subsubsection{Slip-Rotation Lift Force (Mangus Force)}

Particles which are not freely rotating in a flow may also experience a lift force due to the rotation of the particle itself, the so-called Mangus force. High particle rotations may, for example, be induced by inter-particle or particle-wall collisions that frequently occur in pipe or channel flows or by the swirling motion of the surrounding liquid. An analysis expression for the slip-rotation lift force in case of small Reynolds numbers was derived by Rubinow and Keller (1961) as

$$
\mathrm{F}_{\mathrm{LS}}=\pi \mathrm{R}_{\mathrm{P}}^{3} \rho_{\mathrm{F}}\left\{\vec{\Omega} \times\left(\overrightarrow{\mathrm{U}}_{\mathrm{F}}-\overrightarrow{\mathrm{U}}_{\mathrm{P}}\right)\right\}
$$

where relative rotation is given by

$$
\vec{\Omega}=\frac{1}{2} \nabla \times \vec{U}_{\mathrm{F}}-\vec{\omega}_{\mathrm{P}}
$$


Also, the slip-rotation lift force may be extended for higher particle Reynolds numbers by introducing a lift coefficient (Crowe et al., 1998).

$$
\overrightarrow{\mathrm{F}}_{\mathrm{LR}}=\frac{\rho_{\mathrm{F}}}{2} \frac{\pi}{4} \mathrm{D}_{\mathrm{P}}^{2} \mathrm{C}_{\mathrm{LR}}\left|\overrightarrow{\mathrm{U}}_{\mathrm{F}}-\overrightarrow{\mathrm{U}}_{\mathrm{P}}\right| \frac{\vec{\Omega} \times\left(\overrightarrow{\mathrm{U}}_{\mathrm{F}}-\overrightarrow{\mathrm{U}}_{\mathrm{P}}\right)}{\vec{\Omega}}
$$

For lower particle Reynolds number the lift coefficient is obtained according to Rubinow and Keller (1961) in the form:

$$
\mathrm{C}_{\mathrm{LR}}=\frac{\mathrm{D}_{\mathrm{P}}|\vec{\Omega}|}{\left|\overrightarrow{\mathrm{U}}_{\mathrm{F}}-\overrightarrow{\mathrm{U}}_{\mathrm{P}}\right|}=\frac{\operatorname{Re}_{\mathrm{R}}}{\operatorname{Re}_{\mathrm{P}}}
$$

with

$$
\operatorname{Re}_{\mathrm{P}}=\frac{\rho_{\mathrm{F}} \mathrm{D}_{\mathrm{P}}^{2}|\vec{\Omega}|}{\mu_{\mathrm{F}}}
$$

being the Reynolds number of particle rotation. A lift coefficient of higher particle Reynolds number requires experimental information. Recently, Oesterlé and Bui Dinh (1998) introduced the following correlation based on available literature data and additional experiments for $\operatorname{Re}_{\mathrm{P}}<140$ :

$$
\begin{aligned}
& \mathrm{C}_{\mathrm{LR}}=0.45+\left(\frac{\mathrm{Re}_{\mathrm{R}}}{\mathrm{Re}_{\mathrm{P}}}-0.45\right) \exp \left(-0.05684 \cdot \mathrm{Re}_{\mathrm{R}}^{0.4} \cdot \mathrm{Re}_{\mathrm{P}}^{0.3}\right) \\
& \text { for } \operatorname{Re}_{\mathrm{P}}<140
\end{aligned}
$$

The lift coefficient of particle rotation as a function of the particle Reynolds number with the Reynolds number of particle rotation as a parameter is shown in Figure 4.2. 


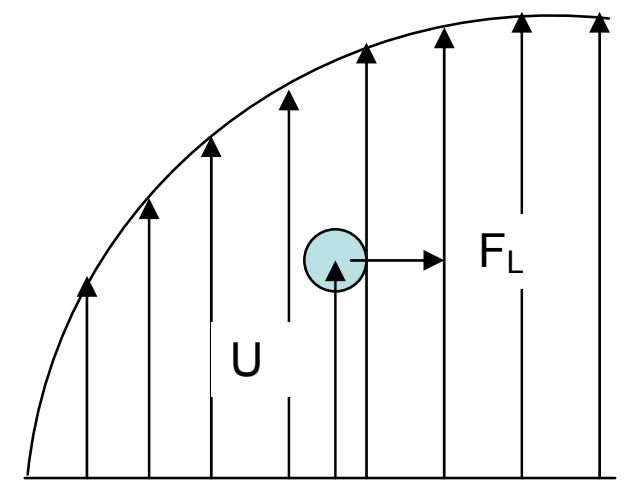

Figure 4.2 Illustration of the slip-shear lift force

\subsubsection{Torque}

The torque acting on a rotating particle due to the interaction with the field was also derived by Rubinow and Keller (1961) for a stagnant fluid and lower particle Reynolds numbers:

$$
\overrightarrow{\mathrm{T}}=-\pi \mu_{\mathrm{F}} \mathrm{D}_{\mathrm{P}}^{3} \vec{\omega}_{\mathrm{P}}
$$

This expression may be extended for a three-dimensional flow and for larger Reynolds numbers by introducing a rotating coefficient:

$$
\mathrm{T}=\frac{\rho_{\mathrm{F}}}{2}\left(\frac{\mathrm{D}_{\mathrm{P}}}{2}\right)^{5} \mathrm{C}_{\mathrm{R}}|\vec{\Omega}| \vec{\Omega}
$$

From the numerical simulations of Dennis et al. (1980), the rotational coefficient for higher particle Reynolds number is found to be :

$$
C_{R}=\frac{12.9}{\operatorname{Re}_{R}^{0.5}}+\frac{128.4}{\operatorname{Re}_{R}} \quad \text { for } 32<\operatorname{Re}_{R}
$$


In the case of smaller particle Reynolds numbers the results of Rubinow and Keller(1961) yields:

$$
C_{R}=\frac{64 \pi}{\operatorname{Re}_{R}} \quad \text { for } \operatorname{Re}_{R}<32
$$

\subsection{Particle-Wall and Particle-Particle Collisions}

In HSBS, vessel and dense suspended solid particles in the fluidized-bed will cause particle-wall and particle-particle collisions, and further affect the mineral separation performance of the HSBS.

The inter-particle collision probability depends mainly on the suspended solid concentration, the particle size, and the fluctuating motion of the particles. In very dilute twophase flows, the particle motion will mainly be governed by fluid dynamic transport effects, i.e. drag force, lift force, and turbulence etc. However, in dense particular flow, particleparticle and wall-particle collisions are unavoidable.

The calculation of the particle linear and angular velocity changes from an inter-particle collision relies generally on the following assumptions:

- Particles are assumed as rigid and spherical

- Only binary collisions are considered, no shape deformation of the particles during the collision process is considered. The interacting forces during the instant particle collision are not considered.

- Particles move in a two-dimension plane, with no linear movement and rotation in the third dimension.

- During the collision calculation, only impulsive forces are considered, and other forces are negligible. 
- Collisions are instantaneous.

\subsubsection{Particle-Wall Collisions}

In a baffled HSBS, the complex flow pattern and structured plate geometry will cause particle-wall or particle-particle collisions and further affect the mineral separation performance.

In the following hard sphere model for the wall collision will be described which implies a negligible particle deformation during the impact process. The change of the particles translational and rotational velocities during the bouncing process can be calculated from the momentum equations of classical mechanics (Crowe et al. 1998). Three types of collisions may be distinguished:

Type 1: The particle stops sliding in the compression period.

Type 2: The particle stops sliding in the recovery period.

Type 3: The particle continues to slide along the wall during the whole collision process.

The type of collision is determined by the static coefficient of friction $\mu_{0}$, the restitution ratio of the normal velocity components, e, and the velocity of the particle surface relative to the contact point, $U_{R 1}$. The non-sliding collision (type 1 and 2) takes place when the following condition is valid:

$$
\begin{gathered}
\left|\mathrm{U}_{\mathrm{R} 1}\right| \leq \frac{7}{2} \mu_{0}(1+\mathrm{e}) \mathrm{V}_{\mathrm{P} 1} \\
\mathrm{U}_{\mathrm{R} 1}=\sqrt{\left(\mathrm{U}_{\mathrm{P} 1}+\frac{\mathrm{D}_{\mathrm{P}}}{2} \omega_{\mathrm{P} 1}^{\mathrm{z}}\right)^{2}+\left(\mathrm{W}_{\mathrm{P} 1}-\frac{\mathrm{D}_{\mathrm{P}}}{2} \omega_{\mathrm{P} 1}^{\mathrm{x}}\right)^{2}}
\end{gathered}
$$

where, $\mathrm{Up}, \mathrm{Vp}$, and $\mathrm{Wp}$ are the translational velocity components and $\omega_{\mathrm{P}}^{\mathrm{x}}, \omega_{\mathrm{P}}^{\mathrm{y}}$, and $\omega_{\mathrm{P}}^{\mathrm{z}}$ are the angular velocity components of the particle in a co-ordinate system as shown in Figure 
4.3. The subscripts 1 and 2 refer to the conditions before and after collision, respectively. For the non-sliding collision, the change of particle velocities is obtained by:

$$
\begin{aligned}
& \mathrm{U}_{\mathrm{P} 2}=\frac{5}{7}\left(\mathrm{U}_{\mathrm{P} 1}-\frac{\mathrm{D}_{\mathrm{P}}}{5} \omega_{\mathrm{P} 1}^{\mathrm{z}}\right) \\
& \mathrm{V}_{\mathrm{P} 2}=-\mathrm{eV}_{\mathrm{P} 1} \\
& \mathrm{~W}_{\mathrm{P} 2}=\frac{5}{7}\left(\mathrm{~W}_{\mathrm{P} 1}+\frac{\mathrm{D}_{\mathrm{P}}}{5} \omega_{\mathrm{P} 1}^{\mathrm{x}}\right) \\
& \omega_{\mathrm{P} 2}^{\mathrm{x}}=\frac{2 \mathrm{~W}_{\mathrm{P} 2}}{\mathrm{D}_{\mathrm{P}}} \\
& \omega_{\mathrm{P} 2}^{\mathrm{y}}=\omega_{\mathrm{P} 1}^{\mathrm{y}} \\
& \omega_{\mathrm{P} 2}^{\mathrm{z}}=-\frac{2 \mathrm{U}_{\mathrm{P} 2}}{\mathrm{D}_{\mathrm{P}}}
\end{aligned}
$$

The type 3 collision is the so-called sliding collision which occurs for:

$$
\left|\mathrm{U}_{\mathrm{R} 1}\right| \geq \frac{7}{2} \mu_{0}(1+\mathrm{e}) \mathrm{V}_{\mathrm{P} 1}
$$

The change of translational and rotational velocities throughout the sliding collision is obtained by:

$$
\begin{aligned}
& \mathrm{U}_{\mathrm{P} 2}=\mathrm{U}_{\mathrm{P} 1}+\mu_{\mathrm{d}} \varepsilon_{\mathrm{x}}(1+\mathrm{e}) \mathrm{V}_{\mathrm{P} 1} \\
& \mathrm{~V}_{\mathrm{P} 2}=-\mathrm{eV}_{\mathrm{P} 1} \\
& \mathrm{~W}_{\mathrm{P} 2}=\mathrm{W}_{\mathrm{P} 1}+\mu_{\mathrm{d}} \varepsilon_{\mathrm{z}}(1+\mathrm{e}) \mathrm{V}_{\mathrm{P} 1} \\
& \omega_{\mathrm{P} 2}^{\mathrm{x}}=\omega_{\mathrm{P} 1}^{\mathrm{x}}-5 \mu_{\mathrm{d}} \varepsilon_{\mathrm{z}}(1+\mathrm{e}) \frac{\mathrm{V}_{\mathrm{P} 1}}{\mathrm{D}_{\mathrm{P}}}
\end{aligned}
$$




$$
\begin{aligned}
& \omega_{\mathrm{P} 2}^{\mathrm{y}}=\omega_{\mathrm{P} 1}^{\mathrm{y}} \\
& \omega_{\mathrm{P} 2}^{\mathrm{z}}=\omega_{\mathrm{P} 1}^{\mathrm{z}}+5 \mu_{\mathrm{d}} \varepsilon_{\mathrm{x}}(1+\mathrm{e}) \frac{\mathrm{V}_{\mathrm{P} 1}}{\mathrm{D}_{\mathrm{P}}}
\end{aligned}
$$

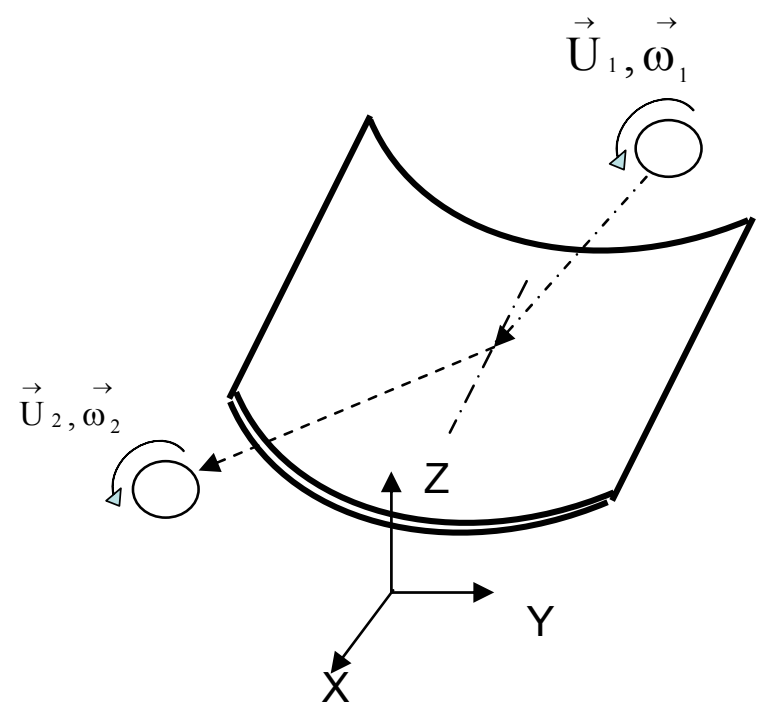

Figure 4.3 Configuration of a particle-wall collision

In Eqs.(4-35) and (4-36) the terms $\varepsilon_{\mathrm{x}}$ and $\varepsilon_{\mathrm{z}}$ determine the direction of the motion of the particle surface with respect to the wall:

$$
\begin{gathered}
\varepsilon_{\mathrm{x}}=\frac{\mathrm{U}_{\mathrm{P} 1}+\frac{\mathrm{D}_{\mathrm{P}}}{2} \omega_{\mathrm{P} 1}^{\mathrm{z}}}{\mathrm{U}_{\mathrm{R} 1}} \\
\varepsilon_{\mathrm{z}}=\frac{\mathrm{W}_{\mathrm{P} 1}+\frac{\mathrm{D}_{\mathrm{P}}}{2} \omega_{\mathrm{P} 1}^{\mathrm{x}}}{\mathrm{U}_{\mathrm{R} 1}}
\end{gathered}
$$

In the above equations $\mathrm{e}$ is the restitution ratio, $\mu_{0}$ and $\mu_{\mathrm{d}}$ are the static and dynamic coefficients of friction (Sommerfeld \& Huber, 1999) 


\subsubsection{Inter-Particle Collisions}

The inter-particle collision (Figure 4.4) probability depends mainly on the particle concentration, the particle size, and the fluctuating motion of the particles. In dilute twophase flows the particle motion will mainly be governed by fluid dynamic transport effects, i.e. drag force, lift force, and turbulence etc. On the other hand, dense flows are characterized by high collision frequencies between particles and hence their motion is dominantly influenced by inter-particle collisions and therefore fluid dynamic transport effects are of minor importance.

The changes of linear and angular velocity components can be calculated by solving the momentum equations. The problem may be further simplified by transforming the particle velocities into a coordinate system where one of the particles is taken as a stationary particle as shown in Figure 4.5. For such a collision geometry, where the relative velocity vector coincides with the axis of the collision cylinder, the relations for the calculation of the postcollision properties of both particles reduce to that for an oblique central collision (Oesterlé \& Petitjean 1994, Sommerfeld 1995). Hence, one obtains the following set of equations to calculate the new linear and angular velocity components of both particles in terms of the relative velocity components before collision:

$$
\begin{array}{ll}
\mathrm{U}_{\mathrm{P} 1}^{*}=\mathrm{U}_{\mathrm{P} 1}+\frac{\mathrm{J}_{\mathrm{x}}}{\mathrm{m}_{\mathrm{P} 1}} & \mathrm{U}_{\mathrm{P} 2}^{*}=-\frac{\mathrm{J}_{\mathrm{x}}}{\mathrm{m}_{\mathrm{P} 2}} \\
\mathrm{~V}_{\mathrm{P} 1}^{*}=\mathrm{V}_{\mathrm{P} 1}+\frac{\mathrm{J}_{\mathrm{y}}}{\mathrm{m}_{\mathrm{P} 1}} & \mathrm{~V}_{\mathrm{P} 2}^{*}=-\frac{\mathrm{J}_{\mathrm{y}}}{\mathrm{m}_{\mathrm{P} 2}} \\
\mathrm{~W}_{\mathrm{P} 1}^{*}=\frac{\mathrm{J}_{\mathrm{z}}}{\mathrm{m}_{\mathrm{P} 1}} & \mathrm{~W}_{\mathrm{P} 2}^{*}=-\frac{\mathrm{J}_{\mathrm{z}}}{\mathrm{m}_{\mathrm{P} 2}}
\end{array}
$$




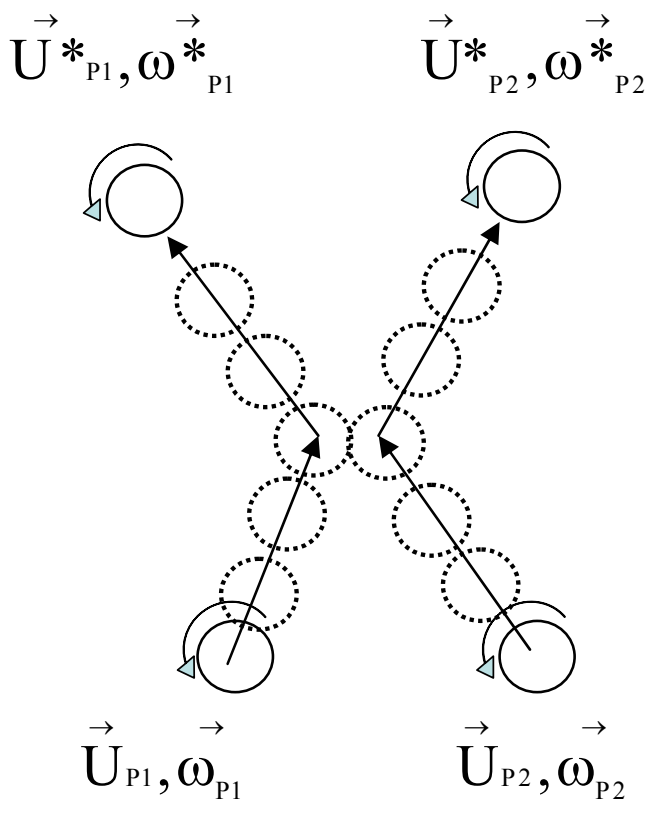

Figure 4.4 Configuration of a particle-particle collision

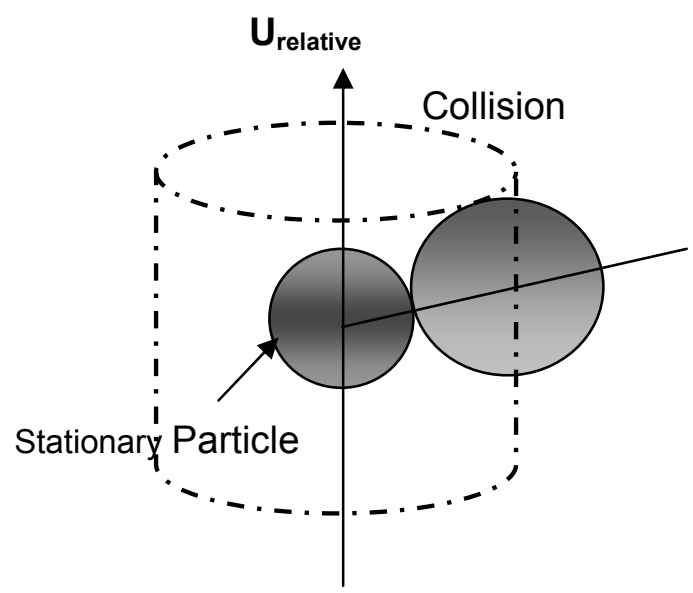

Figure 4.5 Particle-particle collision configurations and co-ordinate system 


$$
\begin{array}{ll}
\omega_{\mathrm{P} 1}^{* \mathrm{x}}=\omega_{\mathrm{P} 1}^{\mathrm{x}} & \omega_{\mathrm{P} 2}^{* \mathrm{x}}=\omega_{\mathrm{P} 2}^{\mathrm{x}} \\
\omega_{\mathrm{P} 1}^{* \mathrm{y}}=\omega_{\mathrm{P} 1}^{\mathrm{y}}-\frac{5 \mathrm{~J}_{\mathrm{z}}}{\mathrm{m}_{\mathrm{P} 1} \mathrm{D}_{\mathrm{P} 1}} & \omega_{\mathrm{P} 2}^{* \mathrm{y}}=\omega_{\mathrm{P} 2}^{\mathrm{y}}+\frac{5 \mathrm{~J}_{\mathrm{z}}}{\mathrm{m}_{\mathrm{P} 2} \mathrm{D}_{\mathrm{P} 2}} \\
\omega_{\mathrm{P} 1}^{* \mathrm{z}}=\omega_{\mathrm{P} 1}^{\mathrm{z}}+\frac{5 \mathrm{~J}_{\mathrm{y}}}{\mathrm{m}_{\mathrm{P} 1} \mathrm{D}_{\mathrm{P} 1}} & \omega_{\mathrm{P} 2}^{{ }_{\mathrm{z}}}=\omega_{\mathrm{P} 2}^{\mathrm{z}}-\frac{5 \mathrm{~J}_{\mathrm{y}}}{\mathrm{m}_{\mathrm{P} 2} \mathrm{D}_{\mathrm{P} 2}}
\end{array}
$$

Here, the superscript $*$ indicates the new velocities after collisions, $\mathrm{m}_{\mathrm{p} 1}$ and $\mathrm{m}_{\mathrm{p} 2}$ are the masses of both particles, $\mathrm{Jx}, \mathrm{Jy}$ and $\mathrm{Jz}$ are the components of the impulsive force. With the definition of the normal restitution ratio:

$$
\mathrm{e}=-\frac{\mathrm{U}_{\mathrm{P} 1}^{*}-\mathrm{U}_{\mathrm{P} 2}^{*}}{\mathrm{U}_{\mathrm{P} 1}}
$$

and the conservation of the $\mathrm{x}$-component of the momentum for particle 2 :

$$
\mathrm{J}_{\mathrm{x}}=-\mathrm{m}_{\mathrm{P} 2} \mathrm{U}_{\mathrm{P} 2}^{*}
$$

One finally obtains the following expression for Jx:

$$
\mathrm{J}_{\mathrm{x}}=-(1+\mathrm{e}) \mathrm{U}_{\mathrm{P} 1} \frac{\mathrm{m}_{\mathrm{P} 1} \mathrm{~m}_{\mathrm{P} 2}}{\mathrm{~m}_{\mathrm{P} 1}+\mathrm{m}_{\mathrm{P} 2}}
$$

By applying Coulomb's law of friction, one obtains the condition for a non-sliding collision as a function of the static coefficient of friction $\mu_{0}$ :

$$
\sqrt{\mathrm{J}_{\mathrm{y}}^{2}+\mathrm{J}_{\mathrm{z}}^{2}}<\mu_{0}\left|\mathrm{~J}_{\mathrm{x}}\right|
$$

Now the components of the impulse force are introduced into Eq.(4-43) and the condition for a non-sliding collision is obtained independent of the velocities of both particles before collision.

$$
\left|\mathrm{U}_{\mathrm{R}}\right| \leq \frac{7}{2} \mu_{0}(1+\mathrm{e})\left|\mathrm{U}_{\mathrm{P} 1}\right|
$$


The relative velocity at the point of contact is determined with the linear and angular velocity components of both particles:

$$
\begin{aligned}
& \mathrm{U}_{\mathrm{R}}=\sqrt{\mathrm{U}_{\mathrm{Ry}}^{2}+\mathrm{U}_{\mathrm{R} z}^{2}} \\
& \mathrm{U}_{\mathrm{Ry}}=\mathrm{V}_{\mathrm{P} 1}+\frac{\mathrm{D}_{\mathrm{P} 1}}{2} \omega_{\mathrm{P} 1}^{\mathrm{z}}+\frac{\mathrm{D}_{\mathrm{P} 1}}{2} \omega_{\mathrm{P} 1}^{\mathrm{z}} \\
& \mathrm{U}_{\mathrm{Rz}}=-\frac{\mathrm{D}_{\mathrm{P} 1}}{2} \omega_{\mathrm{P} 1}^{\mathrm{y}}-\frac{\mathrm{D}_{\mathrm{P} 2}}{2} \omega_{\mathrm{P} 2}^{\mathrm{y}}
\end{aligned}
$$

The components of the impulsive force Jy and Jz are dependent on the type of collision. For a non-sliding collision one obtains:

$$
\begin{aligned}
& \mathrm{J}_{\mathrm{y}}=-\frac{2}{7} \mathrm{U}_{\mathrm{Ry}} \frac{\mathrm{m}_{\mathrm{P} 1} \mathrm{~m}_{\mathrm{P} 2}}{\mathrm{~m}_{\mathrm{P} 1}+\mathrm{m}_{\mathrm{P} 2}} \\
& \mathrm{~J}_{\mathrm{z}}=-\frac{2}{7} \mathrm{U}_{\mathrm{Rz}} \frac{\mathrm{m}_{\mathrm{P} 1} \mathrm{~m}_{\mathrm{P} 2}}{\mathrm{~m}_{\mathrm{P} 1}+\mathrm{m}_{\mathrm{P} 2}}
\end{aligned}
$$

and for a sliding collision the components of the impulsive force are dependent on the dynamic coefficient of friction $\mu_{\mathrm{d}}$ :

$$
\begin{aligned}
& J_{y}=-\mu_{d} \frac{U_{R y}}{U_{R}}\left|J_{x}\right| \\
& J_{z}=-\mu_{d} \frac{U_{R z}}{U_{R}}\left|J_{x}\right|
\end{aligned}
$$

Once the new velocities are obtained they are re-transformed into the original co-ordinate system. The above equations show that the parameters involved in the collision model are the restitution coefficient $\mathrm{e}$, and the static and dynamic coefficients of friction $\mu_{0}$ and $\mu_{\mathrm{d}}$. 


\section{Chapter 5 Boundary Conditions and Numerical Method}

The Euler-Lagrange fluid model, as well as the particle collision sub-model, have been implemented in a computer code written in $\mathrm{C} / \mathrm{C}++$. To summarize the method, the continuous phase flow is obtained by directly solving the Navier-Stokes equation using the SIMPLE (Semi-Implicit Pressure Linked Equations) approach. The motion of each particle is then followed in a Lagrangian frame of reference using the forces generated by fluid motion and gravity. The inter particle collisions and particle-wall collisions are calculated within the same time step for the continuous liquid phase calculation. The effect of particles on the local flow field must then be modeled and fed back into the flow field calculations at each time step. The boundary conditions for each phase and parameters of packing are also incorporated. The correlations between flow dynamics and affecting parameters can be quantitatively analyzed through changing the properties of the particles and the fluid, the geometry of structured plates and the column and operating parameters.

\subsection{Boundary Conditions and Space Grid Size Control}

The boundary conditions are

1) Non-slip conditions for the liquid phase are assumed at the column wall, inlet tube, and outlet tube,

2) The liquid inlet velocity can be specified at the top of the column, for simplicity.

3) The liquid inlet velocity in the feed pipe and the fluidizing water velocity can be specified. 
4) Particles are assumed to be uniformly distributed across the feeding pipe and particles take the velocity of the local liquid velocity. Each particle size and density can be defined from the measured size distribution and density composition of the feed.

5) At the bottom of the column, the heavier materials move freely downward and out of column if the suspension density in the fluidized-bed is above the density setpoint. Otherwise, the bottom of the column is considered as a wall. In this way, the suspension is maintained constant.

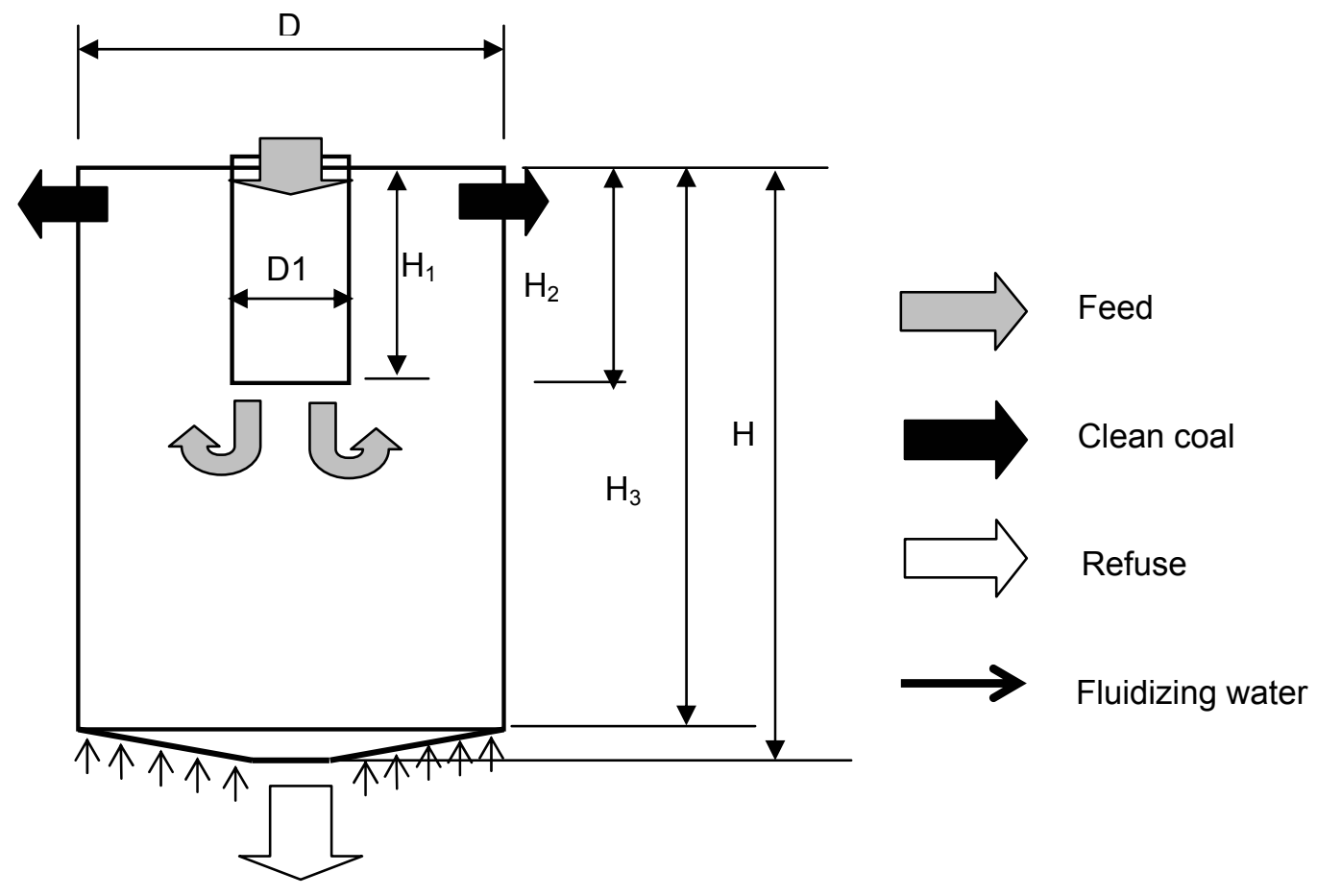

Figure 5.1 HSBS with a center feeding system 
The selection of the space grid will influence the solution obtained. When using a finer grid, the basic flow structure changes little, but it increases the computational cost greatly. Two uniform gird distributions were used, a coarse grid of $30 \times 30$, and a fine grid of $50 \times 50$. The difference in the calculated liquid velocity between coarse grid and fine grid solutions was less than $1 \%$. The fine grid solutions are presented in this paper unless stated otherwise.

\subsection{Calculation of Liquid Phase by SIMPLE Approach}

Finite volume discretization and iterative solutions, based on the SIMPLE algorithm by Patankar (1980), have been used for the numerical solution of the fluid phase. The details of the solution procedures are given by Patankar et al. (1972) and Patankar (1980). The partial differentiate equations for the mass and momentum (Eqs. (3-15) to (3-17)) are solved by using finite differences, derived by integrating the differential equations over the control volumes (Figure 5.2). For this an orthogonal grid is applied, where the two velocities and the pressure are stored in staggered positions (Figure 5.3). Liquid velocity components, $\mathrm{U}$ and $\mathrm{V}$, are stored on the faces of the computation cell, while volume friction and pressure are stored at the grid node points. The necessary linearization of the nonlinear equations is performed by hybrid differences to get an implicit finite difference scheme. Because of the elliptic nature of the partial differential equations, an iterative solution procedure is employed. Starting with initial guessed values for the velocity and pressure fields, the fluid velocities from the momentum equations can be calculated. Therefore, the solution of a pressure correction 


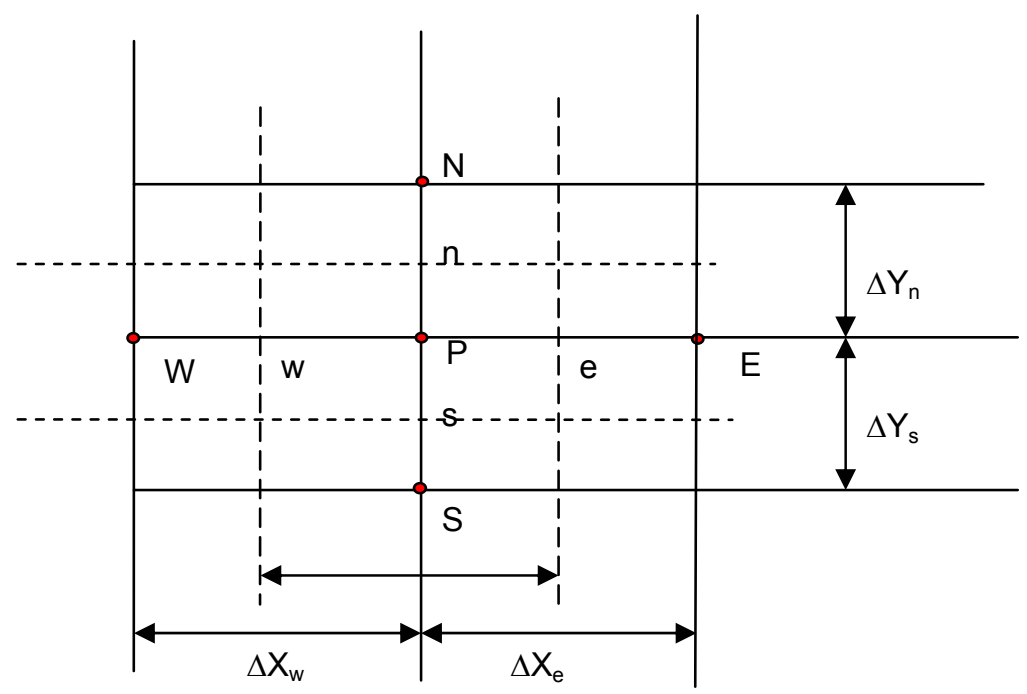

Figure 5.2 Control volume cell

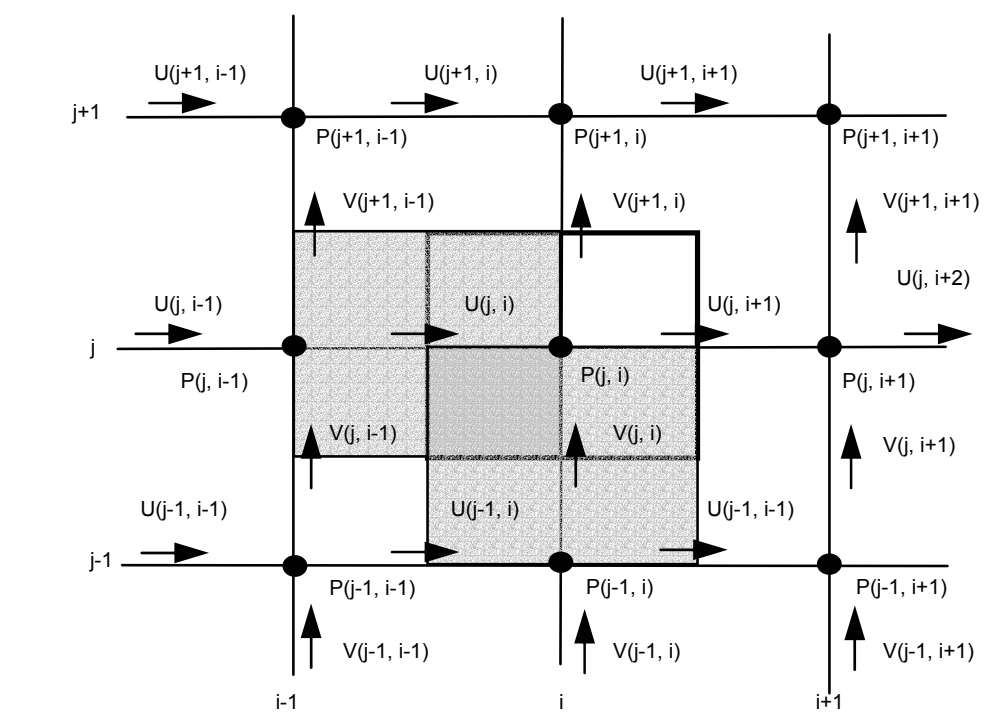

$\rightarrow=\mathrm{U} ; \uparrow=\mathrm{V} ; \bullet=$ other variables (pressure, volume fraction, etc.)

$\mathrm{i}, \mathrm{j}=$ grid point index

Figure 5.3 Staggered locations arrangement of velocity and scalar values over a grid of computation cell 
equation (Poisson equation), which has been derived from the continuity equation yields corrected values for the velocity and pressure fields so that the continuity equation is satisfied. With the corrected values, the momentum equations are solved again and the whole procedure is repeated until convergence is achieved. This was assumed to be the case when the normalized residual at each point, summed over the whole calculation domain, were each smaller than 0.0001 for steady state. The flowchart of the SIMPLE approach is shown in Figure 5.4. The velocities and the location or the liquid volume fractions are obtained by solving the corresponding equations after each iteration of the fluid solution procedure has been carried out. With the computed information on the particles, the momentum transfer terms can be calculated and introduced in the fluid equations before the solution procedure is repeated.

\subsection{Particle Motion Calculation}

In order to handle the interaction between the fluid and the particles, calculations of the particle trajectories by solution of the equations of the motion of the particles, as well as the calculation of the particle source movement transfer terms, is performed in the same iteration loop after the solution of the fluid flow equations, The Runge-Kutta method is used in this study to solve the movement equation of particles and bubbles, update the velocity of particles and bubbles, and further update their position.

The fourth order Runge-Kutta method for the equation $y^{\prime}=f(x, y), y\left(x_{0}\right)=y_{0}$, generates approximations $\mathrm{y}_{\mathrm{n}}$ to $\mathrm{y}\left(\mathrm{x}_{0}+\mathrm{nh}\right)$ for $\mathrm{h}$ fixed and $\mathrm{n}=128$, using the recursion formula

$$
\mathrm{y}_{\mathrm{n}+1}=\mathrm{y}_{\mathrm{n}}+\frac{1}{6}\left(\mathrm{k}_{1}+2 \mathrm{k}_{2}+2 \mathrm{k}_{3}+\mathrm{k}_{4}\right)
$$




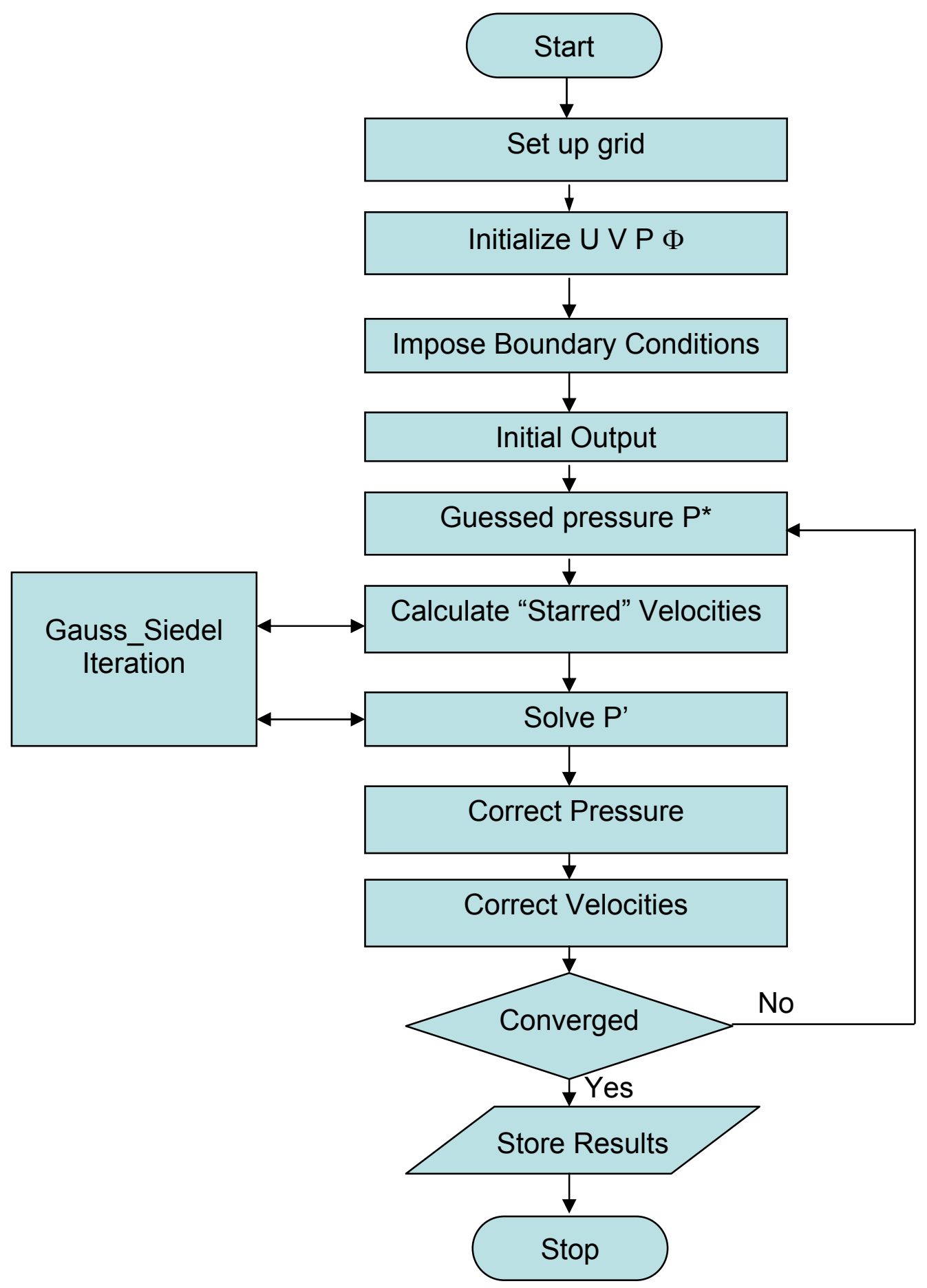

Figure 5.4 Flowchart of application of SIMPLE method 
where

$$
\begin{aligned}
& \mathrm{x}=\mathrm{x}_{0}+\mathrm{nh}, \mathrm{h} \text { is sub-step and } \mathrm{n} \text { is an integer } \\
& \mathrm{k}_{1}=\mathrm{hf}\left(\mathrm{x}_{\mathrm{n}}, \mathrm{y}_{\mathrm{n}}\right) \\
& \mathrm{k}_{2}=\mathrm{hf}\left(\mathrm{x}_{\mathrm{n}}+\frac{\mathrm{h}}{2}, \mathrm{y}_{\mathrm{n}}+\frac{1}{2} \mathrm{k}_{1}\right) \\
& \mathrm{k}_{3}=\mathrm{hf}\left(\mathrm{x}_{\mathrm{n}}+\frac{\mathrm{h}}{2}, \mathrm{y}_{\mathrm{n}}+\frac{1}{2} \mathrm{k}_{2}\right) \\
& \mathrm{k}_{4}=\mathrm{hf}\left(\mathrm{x}_{\mathrm{n}}+\mathrm{h}, \mathrm{y}_{\mathrm{n}}+\mathrm{k}_{3}\right)
\end{aligned}
$$

the local discretization error of Runge-Kutta algorithm is $\mathrm{O}\left(\mathrm{h}^{5}\right)$.

Based on the Runge-Kutta approach, we use four midpoints to obtain an approximation that is correct to fourth order in the time step, i.e., the errors are proportional to $(\Delta \mathrm{t})^{5}$. Of course this greater accuracy is achieved at the price of a total of three intermediate evaluations of the acceleration function instead of just one, thereby requiring considerable more time and programming effort. For calculation of linear movement, we will refer to these three different evaluations of the acceleration by the symbols, $a_{n 1}, a_{n 2}$, and $a_{n 3}$, and the same approach for the associated velocities, $\mathrm{v}_{\mathrm{n} 1}, \mathrm{v}_{\mathrm{n} 2}$ and $\mathrm{v}_{\mathrm{n} 3}$. The midpoint evaluation is as follows

$$
\begin{aligned}
& \left(\frac{d x}{d t}\right)_{n}=v_{n}=v\left[t_{n}, x_{n}, v_{n}\right] \\
& \left(\frac{d v}{d t}\right)_{n}=a_{n}=a\left[t_{n}, x_{n}, v_{n}\right]
\end{aligned}
$$

To obtain $\mathrm{a}_{\mathrm{n}+1}$ and $\mathrm{v}_{\mathrm{n}+1}$, use the procedure described below:

$$
\mathrm{t}_{\mathrm{n}+\frac{1}{2}}=\mathrm{t}_{\mathrm{n}}+\frac{1}{2} \Delta \mathrm{t}
$$




$$
\begin{aligned}
& \mathrm{x}_{\mathrm{n}+\frac{1}{2}}=\mathrm{x}_{\mathrm{n}}+\frac{1}{2} \mathrm{v}_{\mathrm{n}} \Delta \mathrm{t} \\
& \mathrm{v}_{\mathrm{n}+\frac{1}{2}}=\mathrm{v}_{\mathrm{n}}+\frac{1}{2} \mathrm{a}_{\mathrm{n}} \Delta \mathrm{t} \\
& \mathrm{t}_{\mathrm{n}+1}=\mathrm{t}_{\mathrm{n}}+\Delta \mathrm{t} \\
& \mathrm{x}_{\mathrm{n}+1}=\mathrm{x}_{\mathrm{n}}+\mathrm{v}_{\mathrm{n}} \Delta \mathrm{t} \\
& \mathrm{v}_{\mathrm{n}+1}=\mathrm{v}_{\mathrm{n}}+\mathrm{a}_{\mathrm{n}} \Delta \mathrm{t}
\end{aligned}
$$

Then the first midpoint values are:

$$
\begin{aligned}
& \mathrm{a}_{\mathrm{n} 1}=\mathrm{a}\left[\mathrm{t}_{\mathrm{n}+\frac{1}{2}}, \mathrm{x}_{\mathrm{n}+\frac{1}{2}}, \mathrm{v}_{\mathrm{n}+\frac{1}{2}}\right] \\
& \mathrm{v}_{\mathrm{n} 1}=\mathrm{v}_{\mathrm{n}}+\frac{1}{2} \mathrm{a}_{\mathrm{n}} \Delta \mathrm{t}
\end{aligned}
$$

The second midpoint values are:

$$
\begin{aligned}
& \mathrm{a}_{\mathrm{n} 2}=\mathrm{a}\left[\mathrm{t}_{\mathrm{n}+\frac{1}{2}}, \mathrm{x}_{\mathrm{n}}+\frac{1}{2} \mathrm{v}_{\mathrm{n}+\frac{1}{2}} \Delta \mathrm{t}, \mathrm{v}_{\mathrm{n}}+\frac{1}{2} \mathrm{a}_{\mathrm{n} 1} \Delta \mathrm{t}\right] \\
& \mathrm{v}_{\mathrm{n} 2}=\mathrm{v}_{\mathrm{n}}+\frac{1}{2} \mathrm{a}_{\mathrm{n} 1} \Delta \mathrm{t}
\end{aligned}
$$

The third midpoint values are:

$$
\begin{aligned}
& \mathrm{a}_{\mathrm{n} 3}=\mathrm{a}\left[\mathrm{t}_{\mathrm{n}+1}, \mathrm{x}_{\mathrm{n}}+\mathrm{v}_{\mathrm{n} 2} \Delta \mathrm{t}, \mathrm{v}_{\mathrm{n}}+\frac{1}{2} \mathrm{a}_{\mathrm{n} 2} \Delta \mathrm{t}\right] \\
& \mathrm{v}_{\mathrm{n} 3}=\mathrm{v}_{\mathrm{n}}+\mathrm{a}_{\mathrm{n} 2} \Delta \mathrm{t}
\end{aligned}
$$

and then take the average of all those calculated approximations to determine the new updated values: 


$$
\begin{aligned}
& \mathrm{v}_{\mathrm{n}+1}=\mathrm{v}_{\mathrm{n}}+\frac{1}{6}\left\{\mathrm{a}_{\mathrm{n}}+2 \mathrm{a}_{\mathrm{n} 1}+2 \mathrm{a}_{\mathrm{n} 2}+\mathrm{a}_{\mathrm{n} 3}\right\} \Delta \mathrm{t} \\
& \mathrm{x}_{\mathrm{n}+1}=\mathrm{x}_{\mathrm{n}}+\frac{1}{6}\left\{\mathrm{v}_{\mathrm{n}}+2 \mathrm{v}_{\mathrm{n} 1}+2 \mathrm{v}_{\mathrm{n} 2}+\mathrm{v}_{\mathrm{n} 3}\right\} \Delta \mathrm{t}
\end{aligned}
$$

Similar procedures are used for the calculation of particle rotations.

\subsection{Particle Collisions}

Within the constant time step, DT, for the calculation of the liquid phase, all velocities at each grid point are considered constant in accounting for the forces acting on the particles. The directions of velocities of particles do not change until collisions happen. A sequence of collisions is considered one collision a time. It is necessary to find a list of any possible collision pairs. The pair of particles with the smallest collision time is processed first, then the pair with the second smallest collision time, and so on.

The collision time, $\mathrm{T}_{\mathrm{ab}}$, can be calculated from the initial positions and velocities of both particles A and B as shown in Figure 5.5.

$$
\begin{aligned}
& \text { When } \mathrm{Rab} \cdot \mathrm{Vab}>0 \\
& \mathrm{~T}_{\mathrm{ab}}=\frac{-\mathrm{r}_{\mathrm{ab}} \cdot \mathrm{V}_{\mathrm{ab}}-\sqrt{\left(\mathrm{R}_{\mathrm{ab}} \cdot \mathrm{V}_{\mathrm{ab}}\right)^{2}-\mathrm{V}_{\mathrm{ab}}^{2}\left(\mathrm{R}_{\mathrm{ab}}{ }^{2}-\left(\mathrm{R}_{\mathrm{a}}+\mathrm{R}_{\mathrm{b}}\right)^{2}\right)}}{\mathrm{V}_{\mathrm{ab}}^{2}}
\end{aligned}
$$

where $R_{a b} \equiv R_{a}-R_{b}$, and $V_{a b} \equiv V_{a}-V_{b}$.

The algorithms used to process a sequence of collisions within a constant time step of DT is presented in Figure 5.6. Particle-particle interactions are modeled by an impulsive model. The particle-particle collisions and particle-wall collision are calculated at the same time step. Tracking the motion of individual particles within the bed requires 


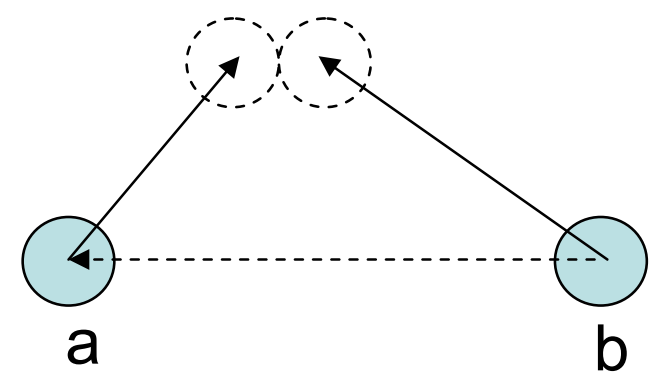

Figure 5.5 Collision of two particles

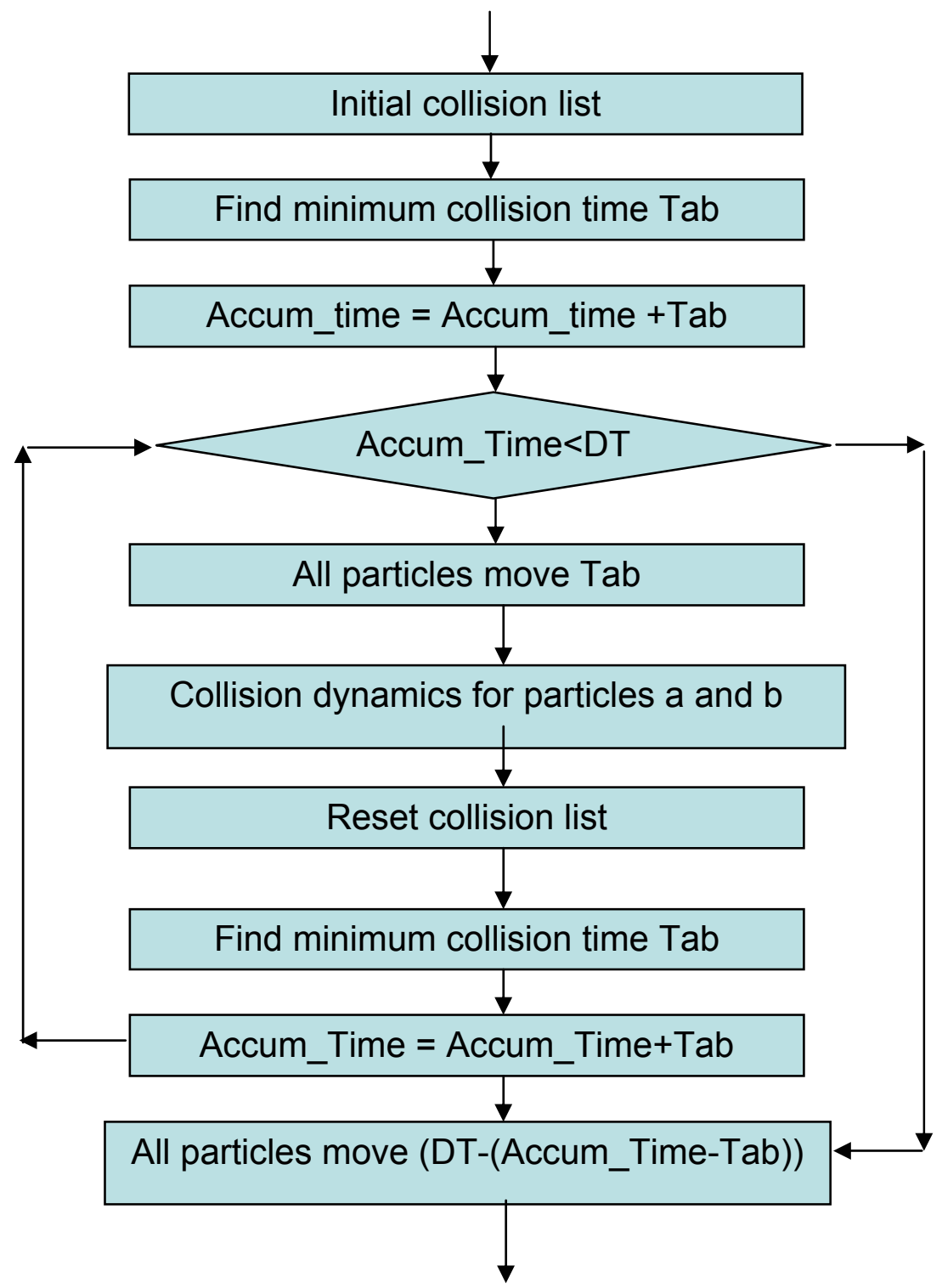

Figure 5.6 Algorithms for the processing of a sequence of collisions within a constant time step DT 
collision checking between the particles. If two particles are heading toward each other within each time step, then the particles are in the process of colliding. Within each time step, the possible collision pairs are found and inserted into a collision list in an order of collision time from the shortest to the longest. Collision checking of each particle could in principle involve looking at all of the remaining particles. This checking time is prohibitive if the number of particle in the bed is huge. An efficient way is needed to obtain acceptable simulation time. In the coding of the simulation, the collision checking of each particle is limited within a certain check distance. Only the particle that will collide with the particle being checked within this distance is listed. This check distance is set as ten times the particle diameter. A similar list is built for collisions between the particles and the walls.

\subsection{Time Step Control}

The Eulerian time step in solving Eqs. (3-15) to (3-20) is selected to be considerably larger that the Lagrangian time step in solving particle movement. For the present computations, the Eulerian time step is selected to be $0.001 \mathrm{~s}$. The Lagrangian time should be less than $10 \%$ of two of following time scales: a) the time for a particle to travel cross a control volume; and b) the smallest particle collision time.

\subsection{Numerical Procedures}

The simulation was coded as a $\mathrm{C} / \mathrm{C}++$ program in which the equations of motion are repetitively solved for each representative particle in the bed. The mathematical model using Euler-Lagrange approach as well as the particle collision sub-model was implemented into a computer code. To summarize the method, the continuous phase flow is obtained by directly solving the Navier-Stokes Equations using the SIMPLE approach. The motion of each 
particle is then followed in a Lagrangian frame of reference using the forces generated by fluid motion and gravity. The equations of motion include forces arising from particle-liquid interactions in a gravity field. The effect of particles on the local flow field must then be modeled and fed back into the flow field for fluid calculations for the next time step. The boundary conditions for each phase and geometry of structured plates are also incorporated. By changing the values of the properties of the particles and the fluid, the geometry of the column and the operating parameters, the correlations between flow dynamics and affecting parameters can be quantitatively analyzed. The overall numerical calculation procedures to solve Navier-Stokes equations by the SIMPLE method are summarized in Figure 5.7. The input to the model involves the knowledge of the particle size and the density distributions in the feed stream. Physical parameters of the HSBS are also required i.e. cell height, cell width, feeding point height, equipment and possible structured plate dimensions. The operating parameters needed for simulation include volumetric feed rate, feed solid concentration, fluidizing water velocity and set point suspension density, etc. The waveform data (shape of waveform, amplitude and frequency) and simulation time are also required for simulation of pulsation flow. 


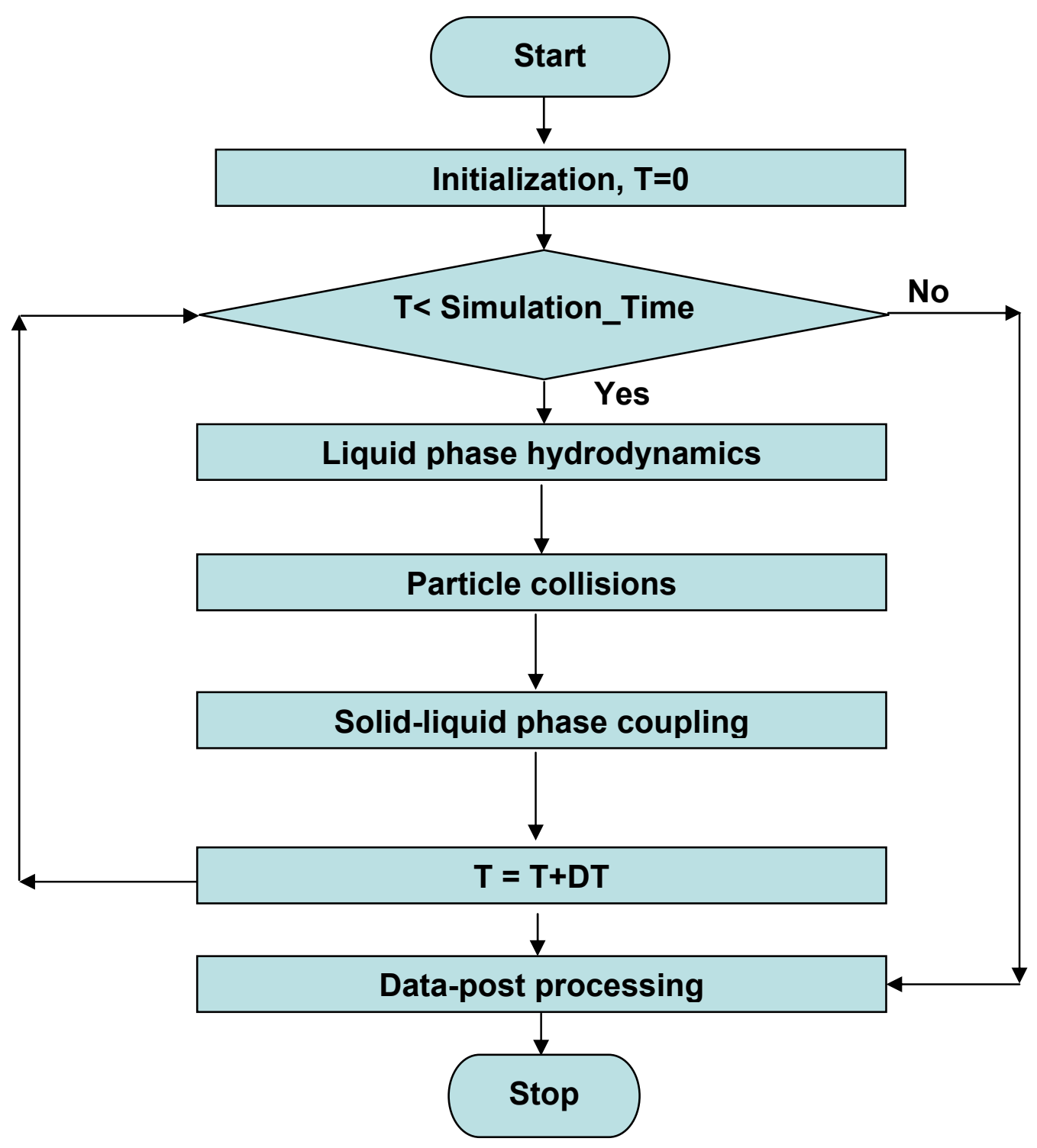

Figure 5.7 Flowchart of principle computational procedures 


\section{Chapter 6 Hindered-Settling Behavior of Particles}

\subsection{Behavior of the Isolated Particle}

The HSBS uses an upward current of water to create a liquid fluidized-bed within the column. A particle with a hindered-settling velocity lower than the upward velocity of the fluidizing water will report to the overflow, otherwise, it will settle down to the underflow as a heavy product. The hindered-settling velocity of a particle is determined by particle properties, such as size and density, and by suspension properties, such as suspension density and viscosity. In order to demonstrate the changes of particle hindered-settling velocity in a hindered-settling process involving a broad range of particle sizes and densities, a few example calculations of isolated particles were made and the results are shown in Figures 6.1 to 6.4. In these figures, a particle with a positive velocity will report to the overflow, and one with a negative velocity will settle down to the underflow. In Figure 6.1, the terminal velocities of particles of different sizes and densities in static water flow were determined. It shows that the terminal velocity of a particle is a function of particle size and its density; it increases with particle size and density in the static liquid phase. Increasing the viscosity of the fluid will reduce the settling speed of a particle. When a viscosity of $10^{-5} \mathrm{~m}^{2} / \mathrm{s}$ for the liquid phase is assumed, the terminal velocity was calculated and is shown in Figure 6.2. Compared to Figure 6.1, the terminal velocities of particles are reduced because of higher drag forces acting on particles in response to the higher viscosity. Increasing the upward current flow velocity 


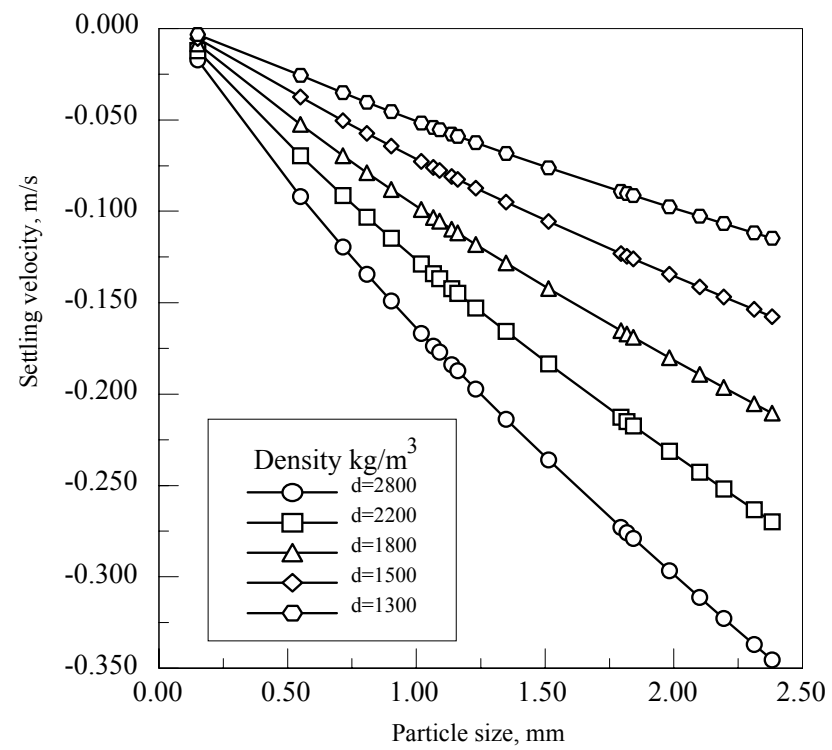

Figure 6.1 Free settling of particles with different sizes and densities (Liquid density: $1.0 \mathrm{~g} / \mathrm{cm}^{3}$, viscosity: $10^{-6} \mathrm{~m}^{2} / \mathrm{s}$ )

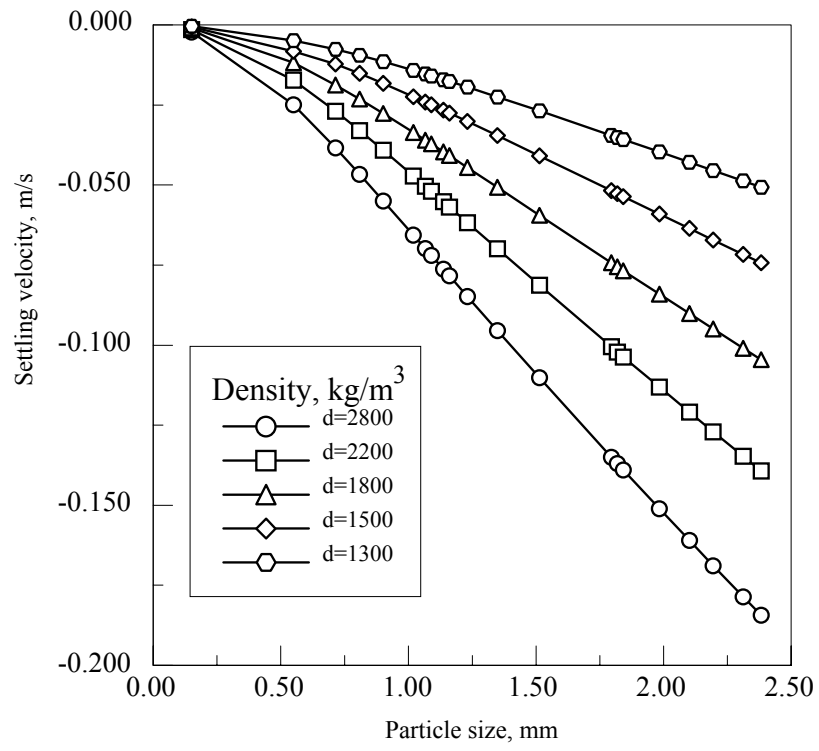

Figure 6.2 Free settling of particles with different sizes and densities (Liquid density: $1.0 \mathrm{~g} / \mathrm{cm}^{3}$, viscosity: $10^{-5} \mathrm{~m}^{2} / \mathrm{s}$ ) 


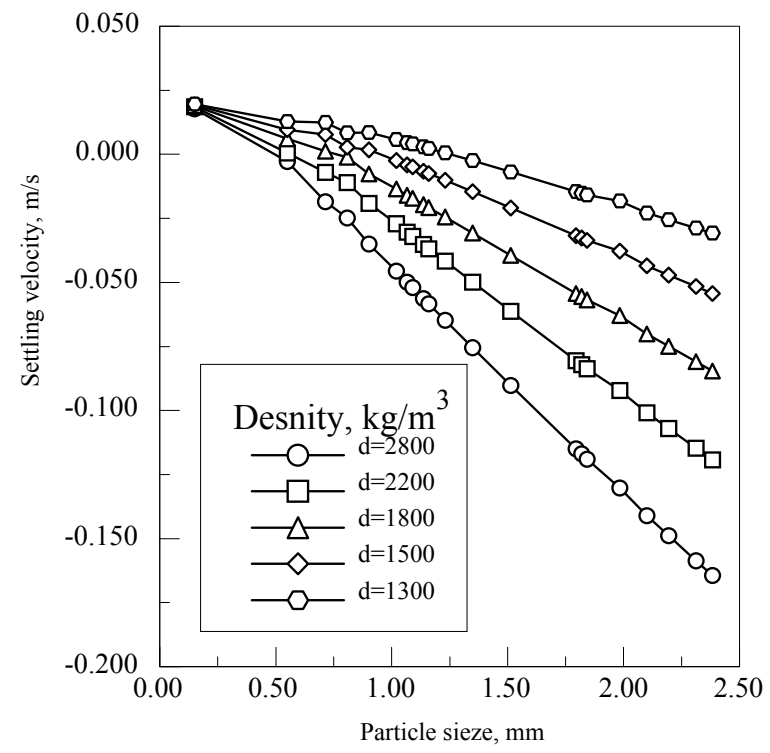

Figure 6.3 Settling of particles with different sizes and densities

(Liquid density: $1.0 \mathrm{~g} / \mathrm{cm}^{3}$, viscosity: $10^{-5} \mathrm{~m}^{2} / \mathrm{s}$,

fluidizing water velocity: $2 \mathrm{~cm} / \mathrm{s}$ )

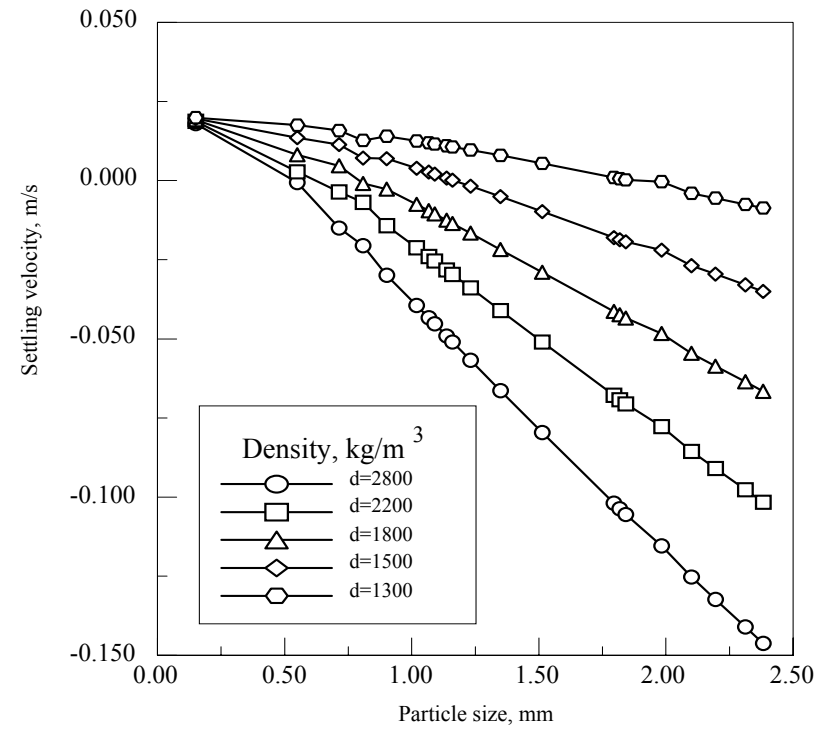

Figure 6.4 Hindered-settling of particles with different sizes and densities

(Suspension density: $1.15 \mathrm{~g} / \mathrm{cm}^{3}$, viscosity: $10^{-5} \mathrm{~m}^{2} / \mathrm{s}$, fluidizing water velocity: $2 \mathrm{~cm} / \mathrm{s}$ ) 
will increase the slip velocity between the particle and the liquid, and further increase the drag forces. In this case, for particles with a radius range of $0.2-2.5 \mathrm{~mm}$ and a density scale of 1.3-2.8 g $/ \mathrm{cm}^{3}$, the terminal velocities of all particles are still negative. That means no particles will move upward until an extra upward current of water is induced. As shown in Figure 6.3 , if an upward teeter water liquid flow $(2 \mathrm{~cm} / \mathrm{s})$ is added, the particles begin to separate. The particles with a positive velocity will float up and report to the overflow, while the particles with negative velocities will settle down to the bottom of the container. The size will also begin to play a role in separation. For particles with a specific density of $1.3 \mathrm{~g} / \mathrm{cm}^{3}$, if the particle size is smaller than $1.5 \mathrm{~mm}$, then it will float up. In the case of a particle with a relative density of $2.8 \mathrm{~g} / \mathrm{cm}^{3}$, only particles finer than $0.25 \mathrm{~mm}$ have a chance to float upward.

The fine, heavy particle will report to the overflow if its size is less than a certain limit. In this case, if the particle radius is finer than $0.25 \mathrm{~mm}$, drag forces acting on these particles are the dominant forces to overcoming the gravity effect, all particles will float upward and the separation is independent of density. On the other hand, if the size of a particle radius is over $1.5 \mathrm{~mm}$, then all particles will settle down because of negligible size effect.

A higher suspension density can cause higher buoyant forces and reduce the settling velocity of the particles. In Figure 6.4, a suspension density of $1.15 \mathrm{~g} / \mathrm{cm}^{3}$ is used. Compared to those curves in Figure 6.3, the effective separation size range is approximately broadened from $-1.5+0.25 \mathrm{~mm}$ to $-2.0+0.25 \mathrm{~mm}$.

\subsection{Particle Collision}

\subsubsection{Particle-Wall Collision}


In a HSBS, at high solid concentration, no particle can move freely in the liquid phase. Particle-particle collisions and particle-wall collisions are unavoidable. When a particle moves near the walls of the HSBS, and it has a normal velocity component toward the wall, it will collide with walls of the container. Figure 6.5 shows a simulated trajectory of a single particle in the process of wall-particle collisions. The particle is injected with a velocity pointing to the vertical wall, after bouncing from wall, it settles down to the bottom by gravity. At the bottom, it keeps bouncing until its kinetic energy dissipates and finally it rests on the bottom of column.

\subsubsection{Natural Packing of Particles}

In order to validate the particle collision model, the settling of a mixture of multiple sized particles was simulated. As shown in Figure 6.6, at initial conditions, 1000 particles are randomly distributed in an area of $100 \times 200$ sq. mm. After 0.4 seconds, all particles settle down by gravity and finally are naturally packed with finer particles inserted in the interspaces of coarse particles. The particles are separated well from each other and from the walls.

\subsection{Importance of the Different Forces}

Analysis of the importance of the different forces acting on a particle in the solid-liquid fluidized-bed system is performed for different sized particles and for particles with different densities in a baffled column without pulsation flows. The detail structure of the baffled column is shown in Figure 8. 2. 


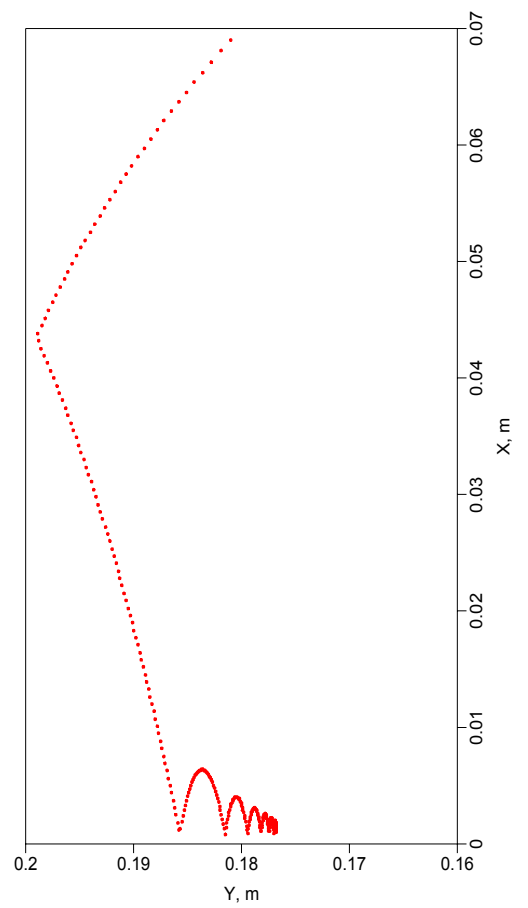

Figure 6.5 Simulation of trajectory wall-particle collision (Initial position $(\mathrm{x}, \mathrm{y})=(0.07,0.18)$, and initial velocity $\mathrm{V}(\mathrm{x}, \mathrm{y})=(-0.5,0.5)$ in static water; Density $=2.7 \mathrm{~g} / \mathrm{cm}^{3}$; Size $=2 \mathrm{~mm}$; Restitution ratio $=0.95$; static and dynamic friction coefficient $=0.02$ )

The result of this analysis is shown in Figure 6.7 and 6.8. In Figure 6.7 particles have a uniform size but with a relative density composition range from 1.2 to 2.8 . The fluidizing water velocity is $25.2 \mathrm{~m}^{3} / \mathrm{m}^{2} \mathrm{~h}$ and the solid concentration is about $15 \%$. The different forces are compared in Figure 6.7 with changes of densities at an instant time $t=30$ seconds. It was shown that the drag force, pressure and buoyancy force are dominant forces by some factors of magnitude. The drag force, pressure and buoyancy a force have an order of $10^{-6} \mathrm{~N}$. The other forces are of order $10^{-8} \mathrm{~N}$. These main forces have nearly linear relationship with particle densities. The Saffman force has the smallest magnitude order among all forces. Added mass force and Magnus force are about two orders smaller than drag force. 
$\mathrm{T}=0$

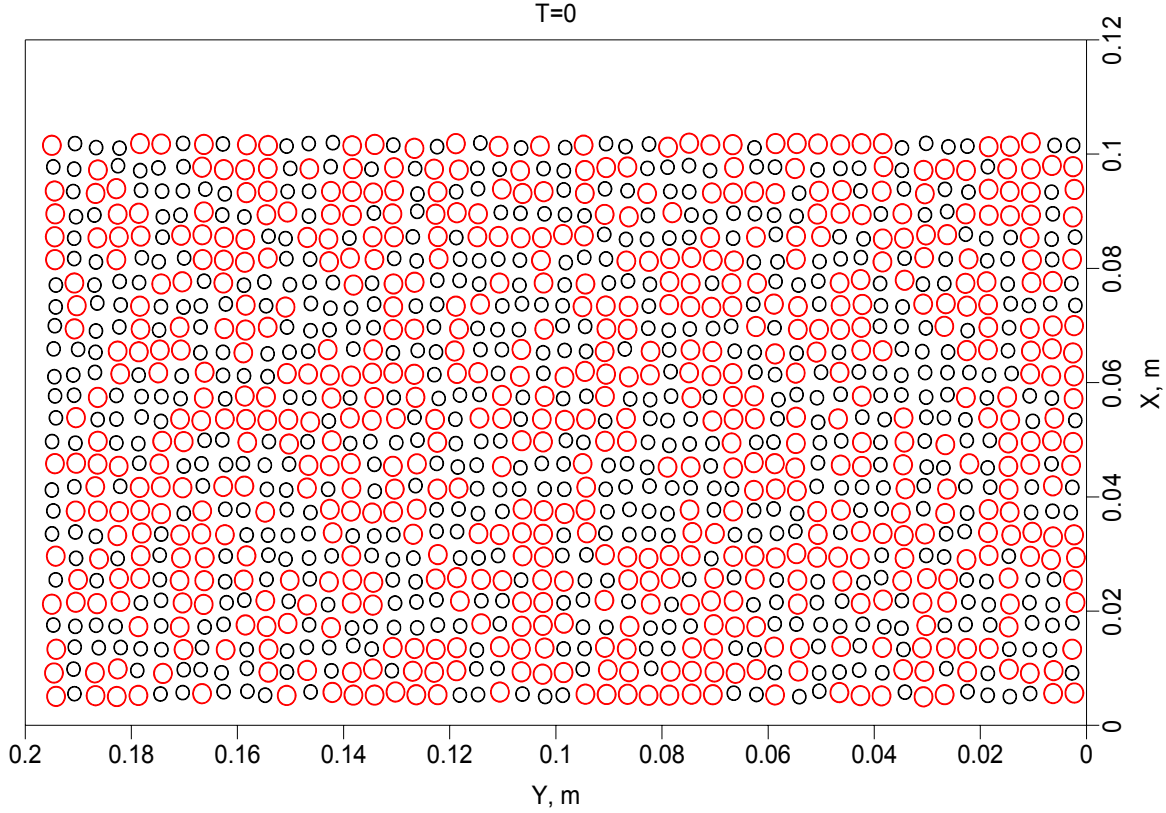

(a)

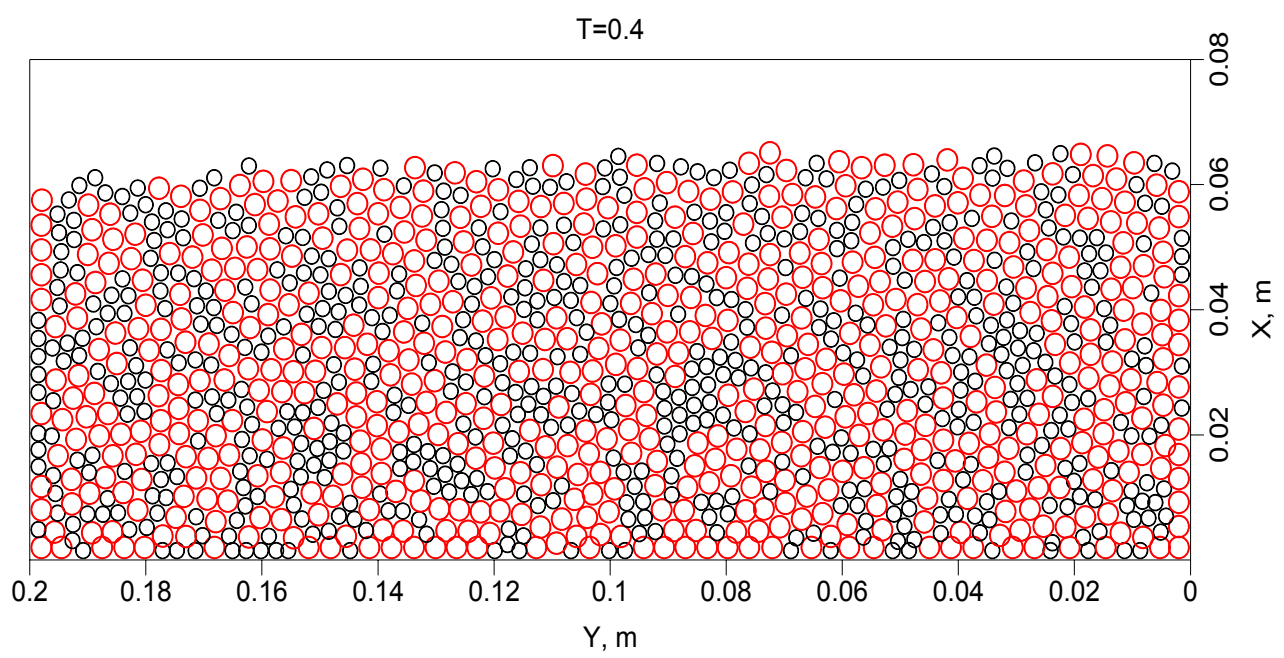

(b)

Figure 6.6 The natural packing of particles with different sizes

(Diameter: $2 \mathrm{~mm}$ and $1 \mathrm{~mm}$; Density $1.5 \mathrm{~g} / \mathrm{cm}^{3}$ ) 


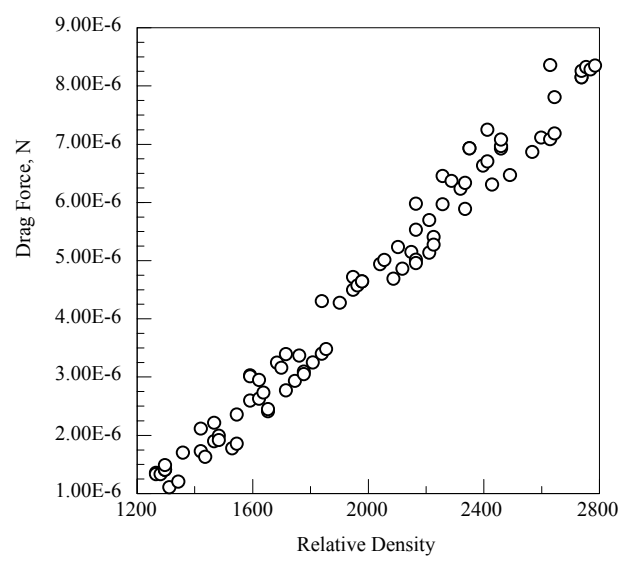

Drag Force

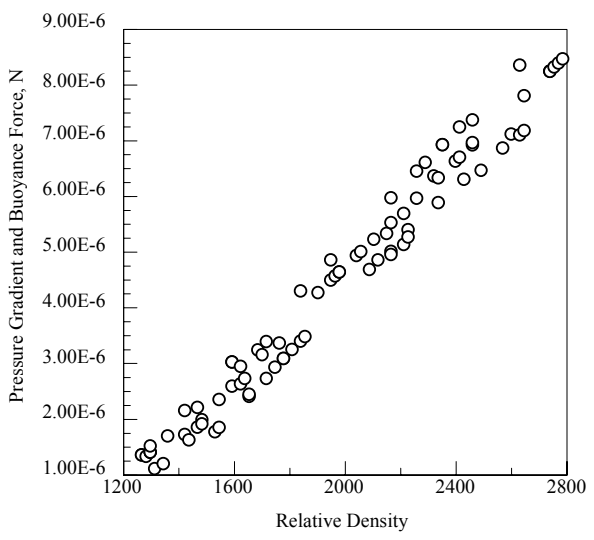

Pressure Gradient and Buoyancy Force

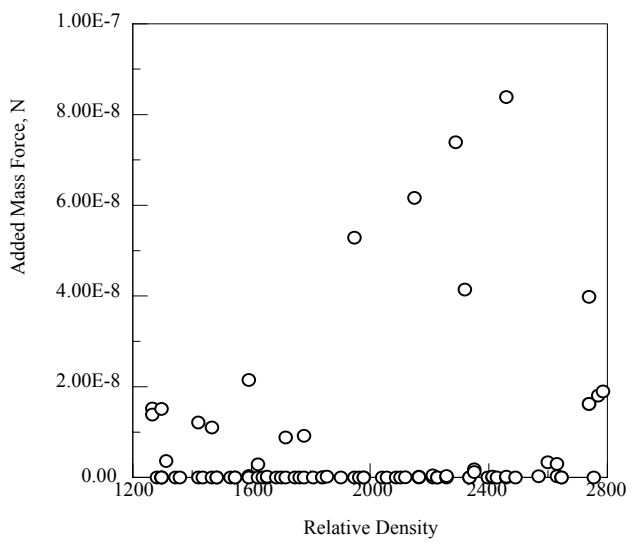

Added Mass Force

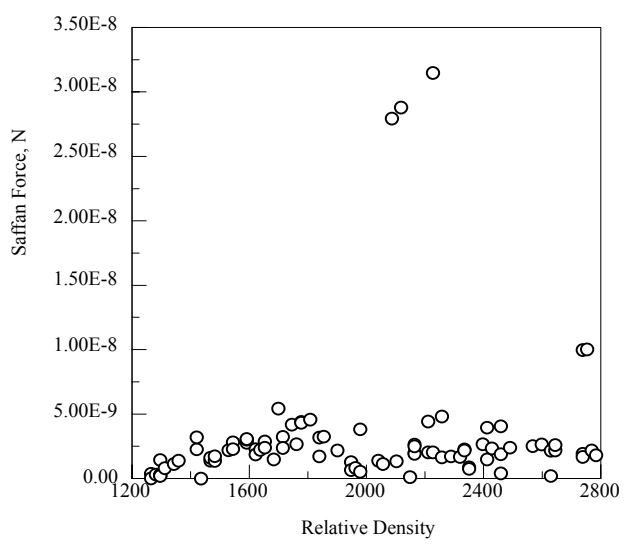

Saffman Force (Slip-Shear Lift Force)

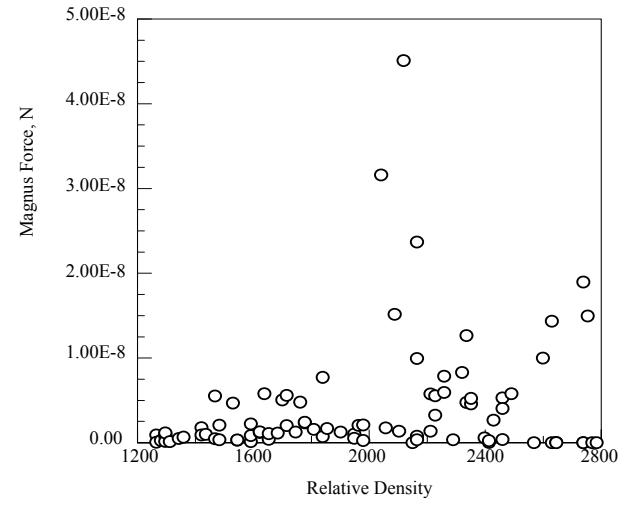

Mangus Force (Slip-Rotation Lift Force)

Figure 6.7 Importance of the different forces with densities for uniform sized particles 


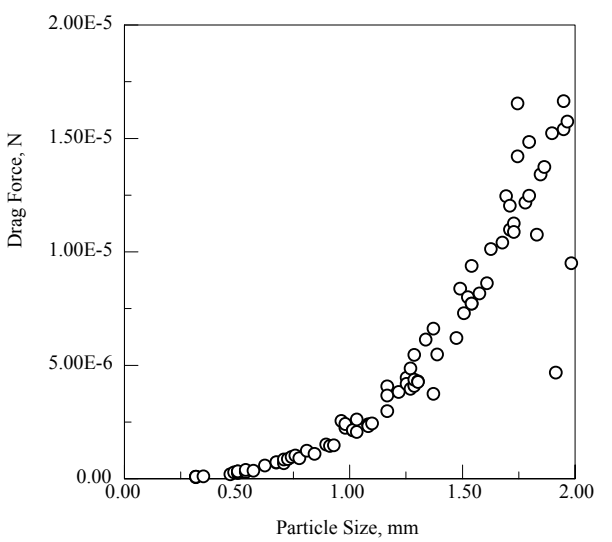

Drag Force

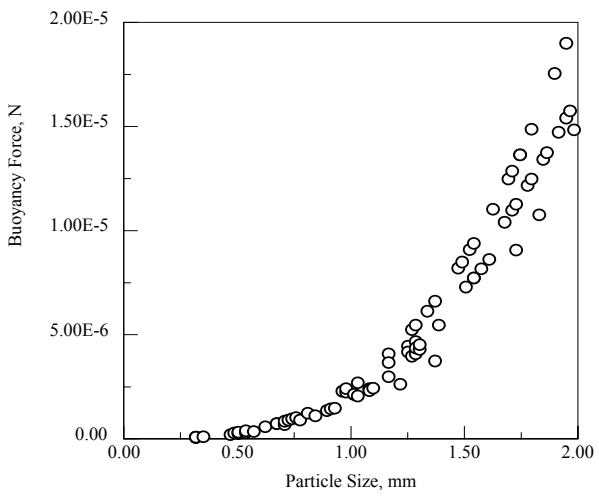

Pressure Gradient and Buoyancy Force

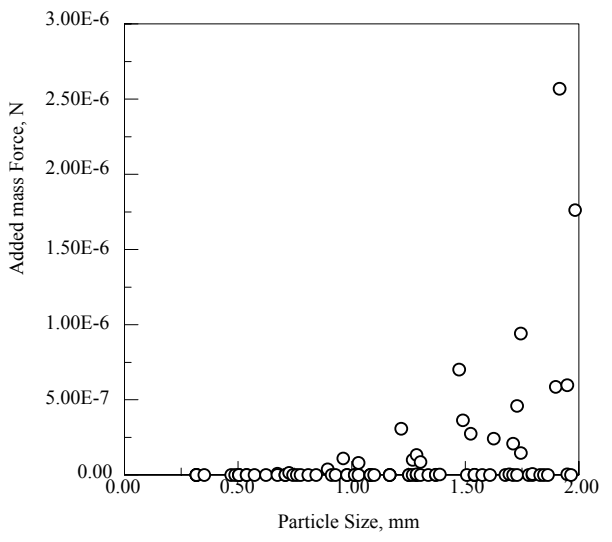

Added Mass Force

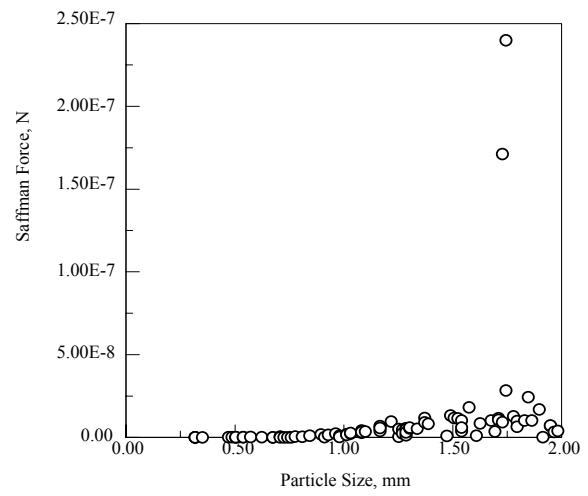

Saffman Force (Slip-Shear Lift Force)

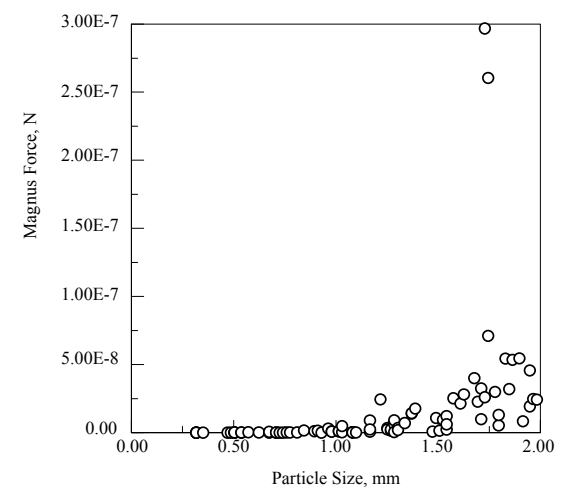

Mangus Force (Slip-Rotation Lift Force)

Figure 6.8 Importance of the different forces with sizes for the particles with constant density 
The relative magnitudes of different forces are compared in Figure 6.8. In this case, all particles have a constant relative density value of 1.5 and other parameters are kept constant. The size range is $-2.0+0.2 \mathrm{~mm}$. As shown in Figure 6.8, the drag force, pressure gradient and buoyancy forces are still the dominant forces with an exponential relationship with sizes. The Saffman force is about three orders less than drag force. The added mass force and the Magnus forces are two orders smaller than drag force. However, it is should be noted that added mass weight increase to about $10 \%$ of the drag force for some particles.

Comparing Figure 6.7 and Figure 6.8, it is obvious that size has a stronger effect on changes of different forces than density does. Overall, the Magnus force, the Saffman force and the added mass forces are relatively small. But in the baffled column, especially in some dented area of structured plates where liquid flow rotation exists, these forces might increase to by one or more orders in magnitude. In uniform flows where no velocity gradient exists, these forces may be neglected without considerable error.

In this study, all six forces are kept in the computations since some high velocity gradients exist in the HSBS and in the baffled columns with structured plates even though the computation costs increases slightly.

\subsection{Particle Stratification in Pulsating Flows}

The Euler-Lagrange model from the Computational Fluid Dynamics approach is used to simulate coal stratification in pulsating flow. Stratification is a phenomena of particle separation in which particles are layered based on density differences. Figure 6.9 shows the

pulsating movement of three discrete coal particles with different densities in a pulsating flow with a sinusoidal wave. The heavy particle moves to the bottom of column while the 
light particle moves to the top of column. In Figure 6.10, the stratification of a group of coal particles due to density differences in pulsating liquid flows is simulated.

The simulation displays the characteristics of the jigging process. At the beginning $(\mathrm{t}=0)$, the particles are distributed randomly, with a uniform size and a density distribution from 1.3 to $2.8 \mathrm{~g} / \mathrm{cm}^{3}$ (Figure 6-10a). The particles were pulsated in a $100 \times 100 \mathrm{sq} . \mathrm{mm}$ zone where the particles occupy a bed height approximately $3.5 \mathrm{~cm}$ when bed is at rest. Pulsation of the jig bed was carried out by applying a sinusoidal jig cycle where amplitude and frequency were fixed at $1.5 \mathrm{~cm}$ and $2 \mathrm{~Hz}$, respectively. Three snapshots of the graphical display are shown in Figure 6.10. With time, in a pulsating flow, light particles experience a higher buoyancy force than heavier particles. In the upward stroke (Figure 6.10b), light particle moves to a higher position than heavier particles. In the downward flow, the heavier particles settle faster than the light particles. The particles which have a medium density have an equivalent probability to be mixed in both upper layer clean coal and bottom layer refuse because of particle collisions. At time $\mathrm{t}=3.5$ seconds, the particles were stratified successfully with light particles on the top of the particle body and heavy particle layered near the bottom (Figure 6.10c). The effects of pulsation frequency and amplitude on particle separation are also explored to find the optimal values for best stratification in the following chapters. 


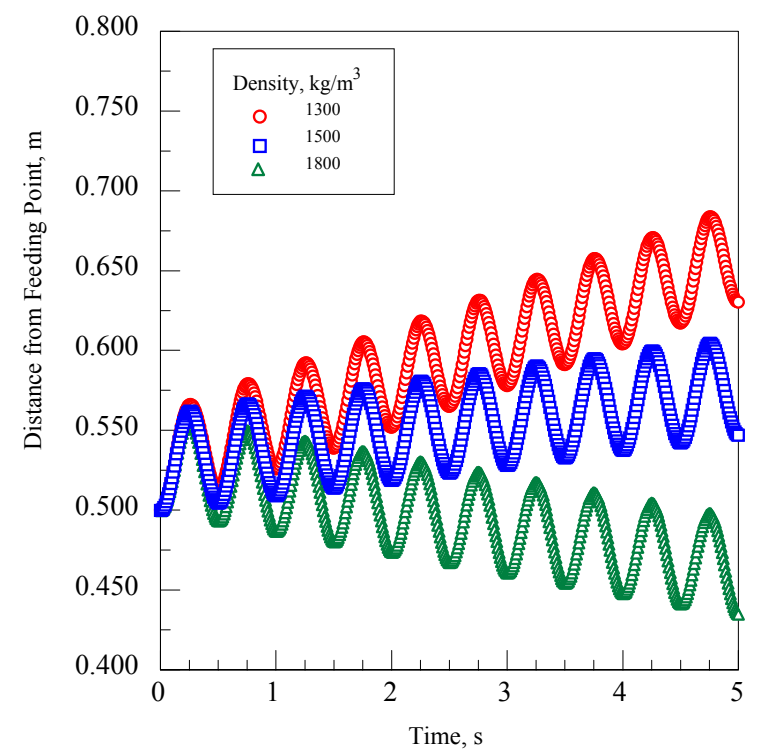

Figure 6.9 Fluctuating movement of particles in a vertical pulsating flow (Particle size: $1 \mathrm{~mm}$, suspension density: $1.15 \mathrm{~g} / \mathrm{cm}^{3}$, viscosity: $10^{-5} \mathrm{~m}^{2} / \mathrm{s}$, frequency of pulsation: $2 \mathrm{~Hz}$, amplitude of pulsation: $1.5 \mathrm{~cm} / \mathrm{s}$, fluidizing water velocity: $2 \mathrm{~cm} / \mathrm{s}$ )

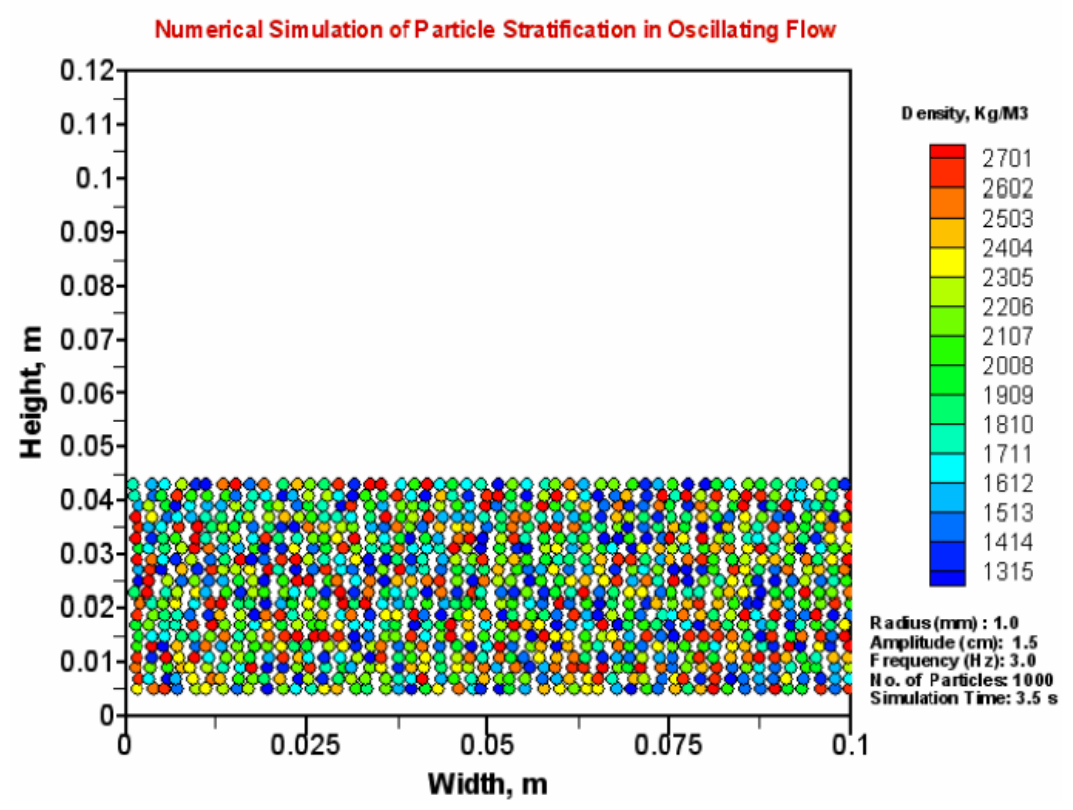

A. $\mathbf{t}=\mathbf{0} s$ 


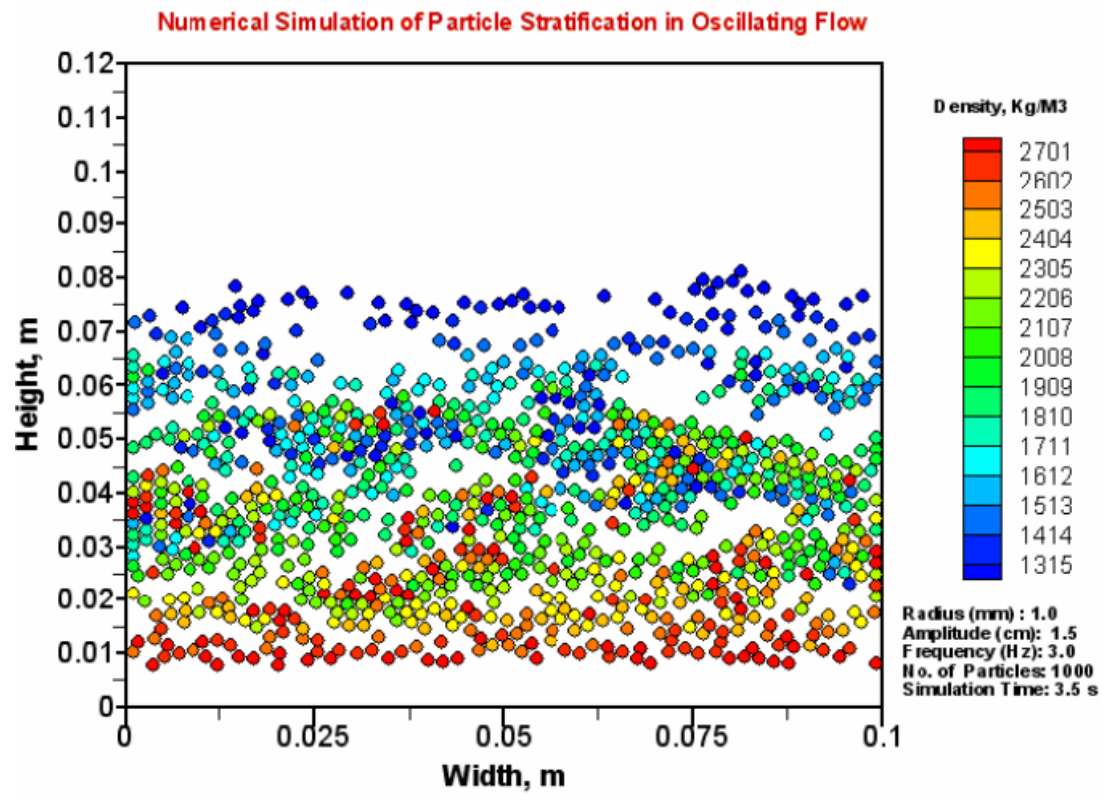

B. $\mathrm{t}=\mathbf{2 s}$

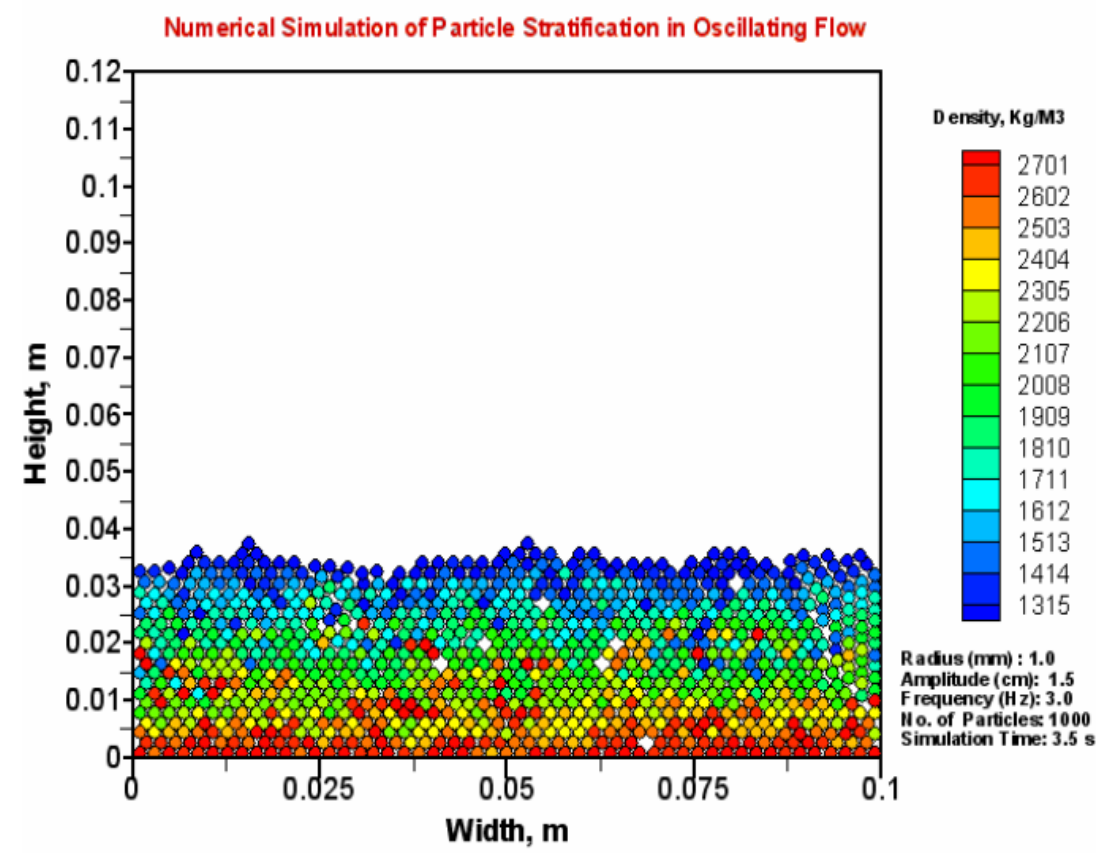

C. $t=10 s$

Figure 6.10 Stratification of fine coal particles $(t=3.5 \mathrm{~s})$ 


\subsection{Solid-Liquid Coupling}

In order to demonstrate the solid-liquid coupling effect, we present numerical results for flow of solid-liquid mixtures in a vertical container with a width of $0.2 \mathrm{~m}$ and a height of 1.0 m. The simulation results are shown in Figures 6.11 and 6.12. All particle velocities are set equal to zero in a static liquid at the beginning of the simulation and the time of step (DT) for the liquid phase calculation was chosen to be $10^{-4} \mathrm{~s}$. As shown in Figure 6.11 (a), the 400 particles have a uniform density (specific gravity: 2.0) and the particles are circular with a uniform diameter $(2 \mathrm{~mm})$. Initially, all particles were randomly positioned in which particles are circular with a uniform diameter $(2 \mathrm{~mm})$. Initially, all particles were randomly positioned in a container of $200 \times 400$ sq. $\mathrm{mm}$ area and the fluid was static at beginning. The gravity is directed down the column walls and drives the particular flow. The restitution coefficient for particle collision is set equal to 0.5 and the friction coefficient set as 0.02 . The number of the particles in the computational cell stays fixed during the simulation. Particles settle downward and accumulate gradually at the bottom in a lattice pattern

During the initial transient movement, the particles fall and the bulk of the fluid flows are dragged downward by the coupling forces from the settling particles. The free settling of isolated particles is also disturbed by local liquid flow and results in particle-particle and particle-wall collisions. The particles are normally well separated from each other and from the column walls. During the sedimentation of the particles, long particle chains are formed along flow paths. The particle-particle interactions and wakes formed behind particles can draw particles together and form particle clusters. As shown in Figure 6.12 (a), at the beginning of the hindered-settling $(\mathrm{T}=0.02 \mathrm{~s})$, an overall circulation of liquid flow is formed 


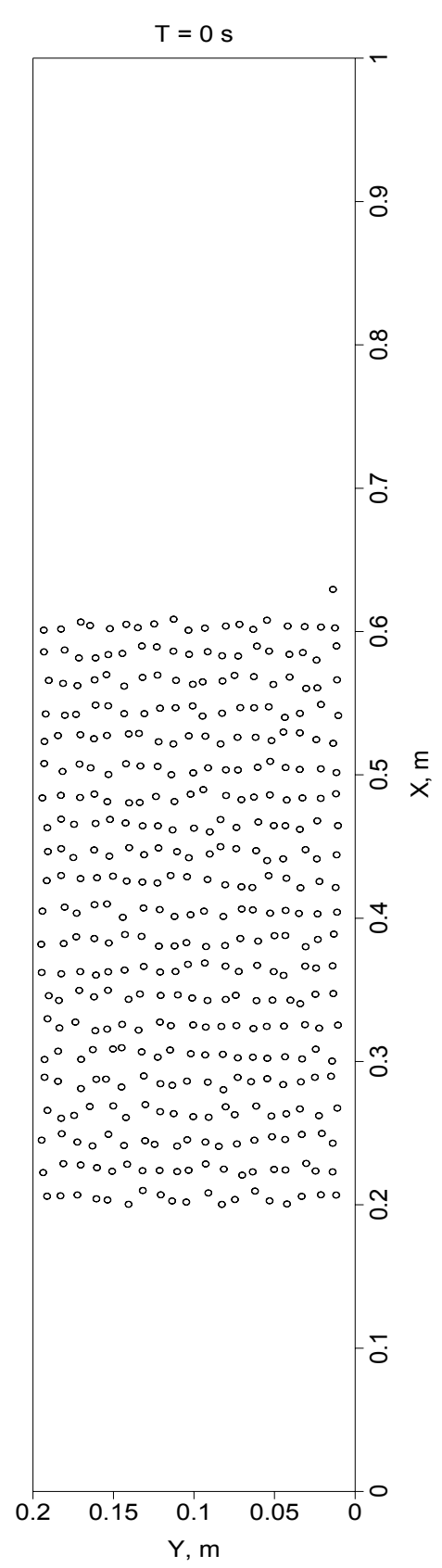

(a)

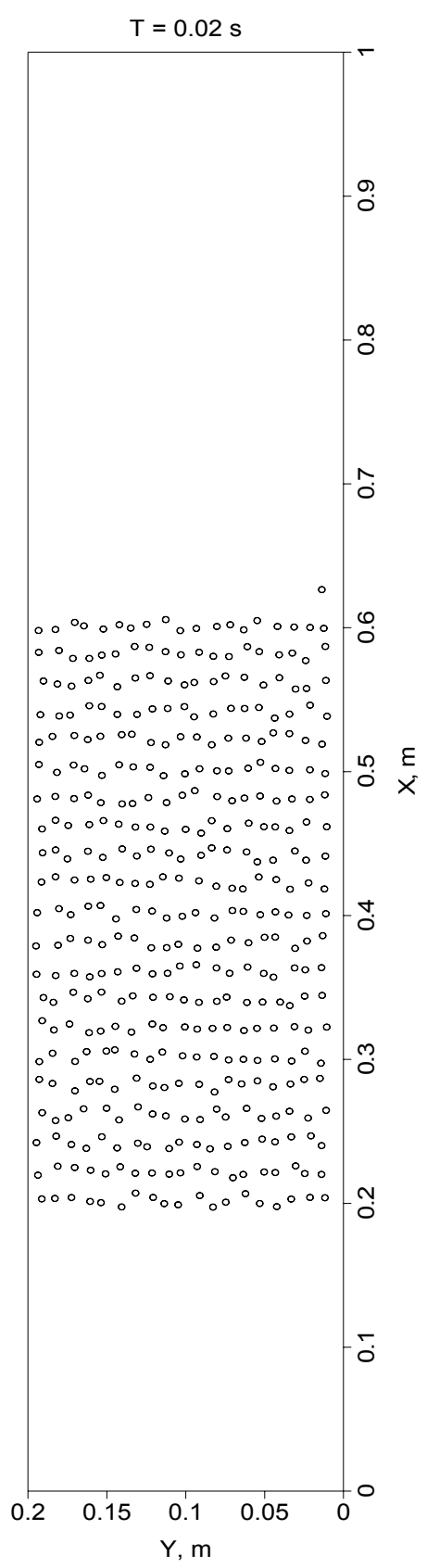

(b)

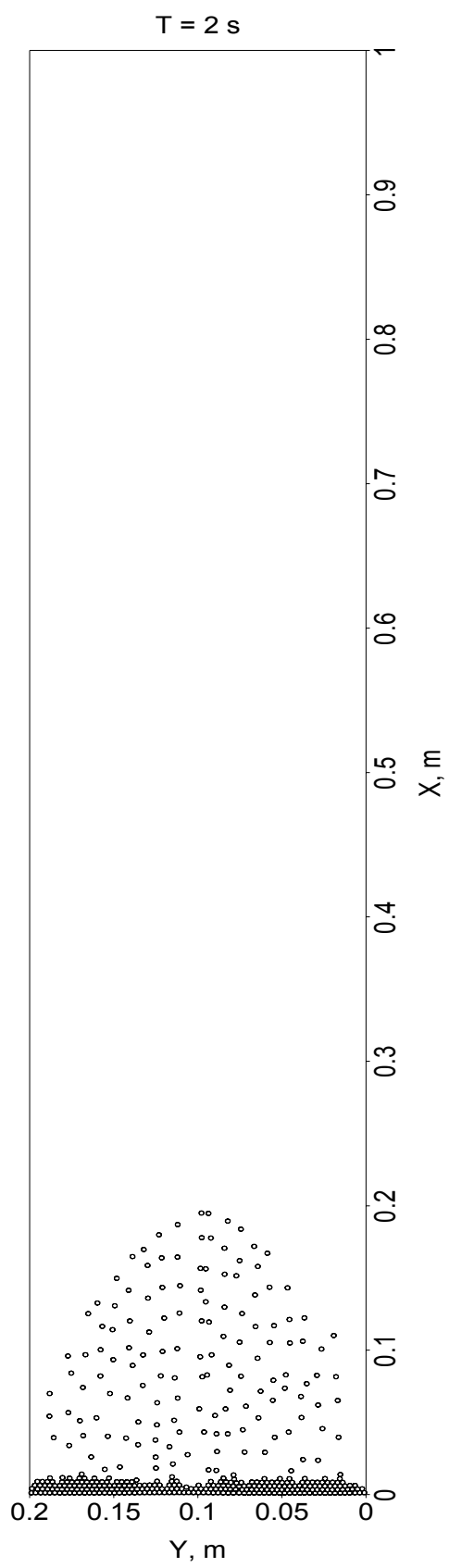

(c)

Figure 6.11 Hindered-settling of 400 particles in an initially static liquid flow 


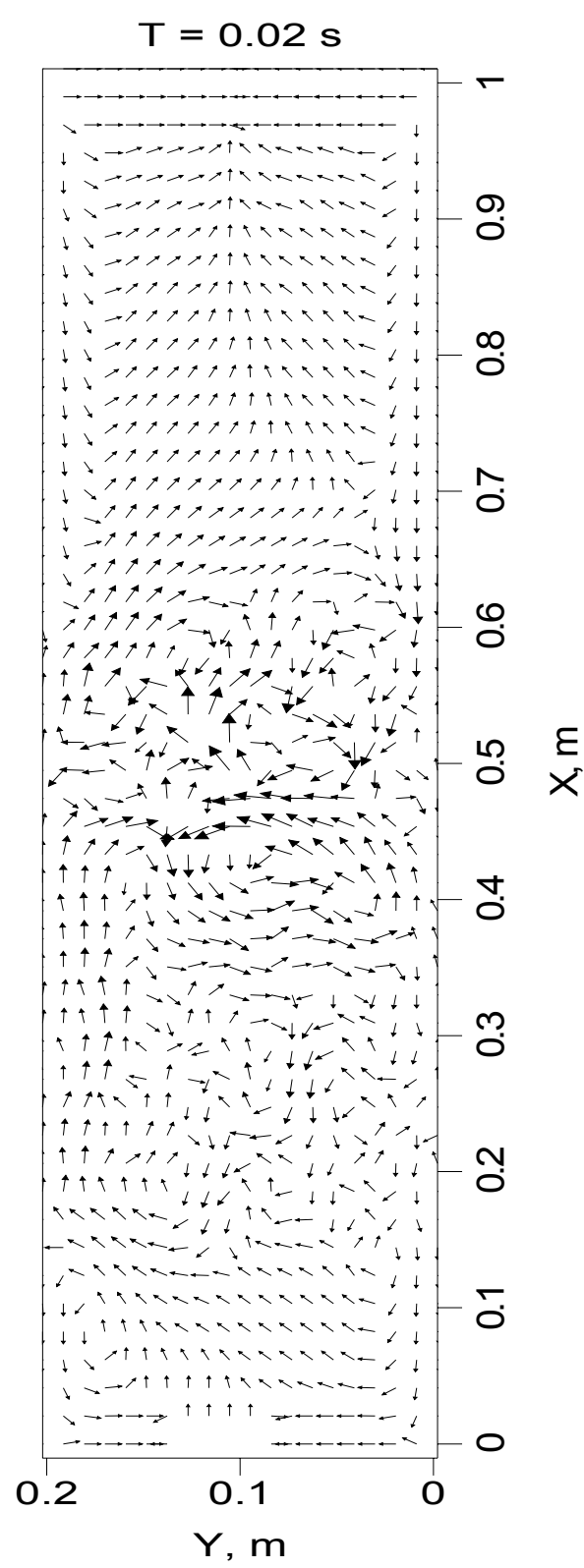

(a)

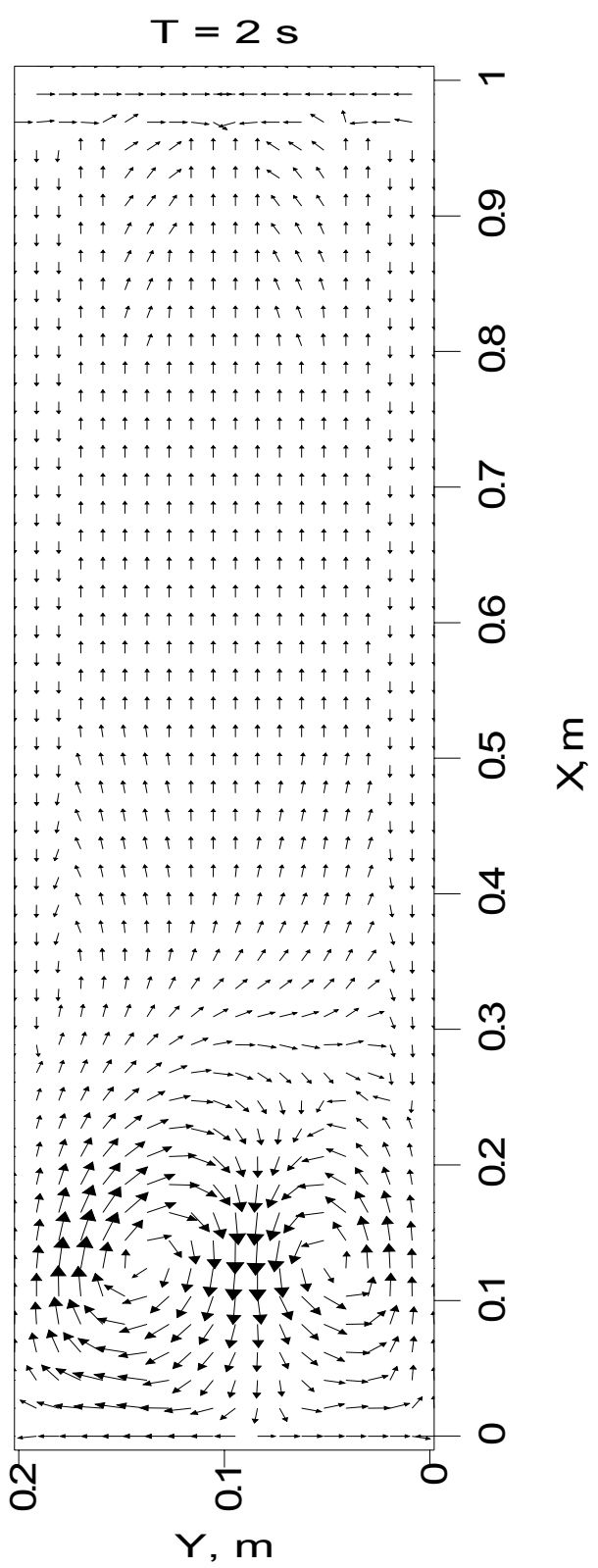

(b)

Figure 6.12 The instant liquid phase velocity vector maps during particle settling

above the particle group body with upward flow in the middle of the column and downward flow near the walls of the column. The liquid pattern below the particle body shows 
asymmetric characteristics with downward flow near one side of the wall and upward flow on the other side of the wall. Within the bulk body of particles, the liquid flow is very complicated, and a few vortices are randomly formed. At time $t=2 \mathrm{~s}$, nearly all particles have reached the bottom of the column. The flow in the middle of the column was forced downward by the particles with upward flow near the walls. The liquid pattern above the particles shows approximately symmetric characteristics with upward flow in middle of the column and downward flow near the walls. Eventually, after all particles reach the bottom of the column, the liquid flow returns back to the static state and all vortices disappear.

\subsection{Effect of Particle Properties on Particle Separation}

The effect of particle size and density on the settling of particles is checked in a channel with a width of $0.1 \mathrm{~m}$ and a height of $1 \mathrm{~m}$ in three cases. The particles have a weight evenly distributed among sizes between $0.2 \mathrm{~mm}$ and $5 \mathrm{~mm}$. In the first case, the particle density is fixed at $1.5 \mathrm{~g} / \mathrm{cm}^{3}$, an upward uniform fluidizing fluid velocity $(3 \mathrm{~mm} / \mathrm{sec})$ is applied while keeping the other parameters constant. The initial distribution of the particles is kept in an area of $100 \times 200$ sq. $\mathrm{mm}^{2}$ as shown in Figure 6.13 (a). At the initial transient time, the fine particles begin to move upward and coarse particles move downward. The particles with a medium size ( 2 to $3 \mathrm{~mm}$ ) have a nearly zero slip velocity relative to the liquid phase, neutrally float with liquid flow and tend to accumulated in the channel. The most interesting feature for upward flow is that the small particles have a trend to migrate away from the wall region if the particle is denser than the liquid. This migration effect is attributed to the Saffman force effect in the particle motion equation, which points toward the channel axis when heavy particles move in an upward flow. 


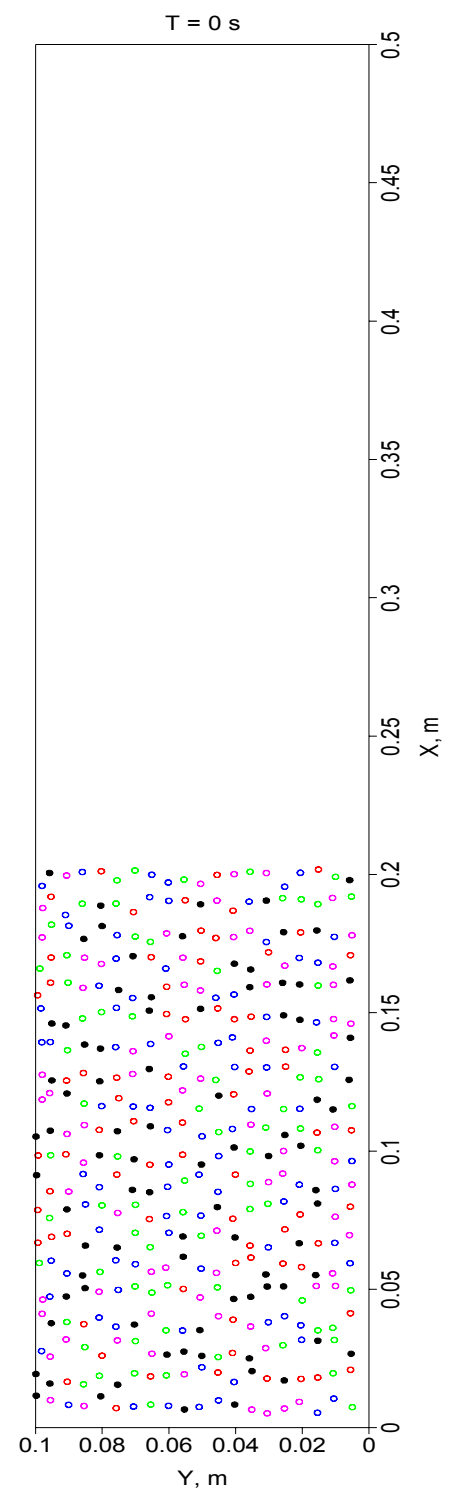

(a)

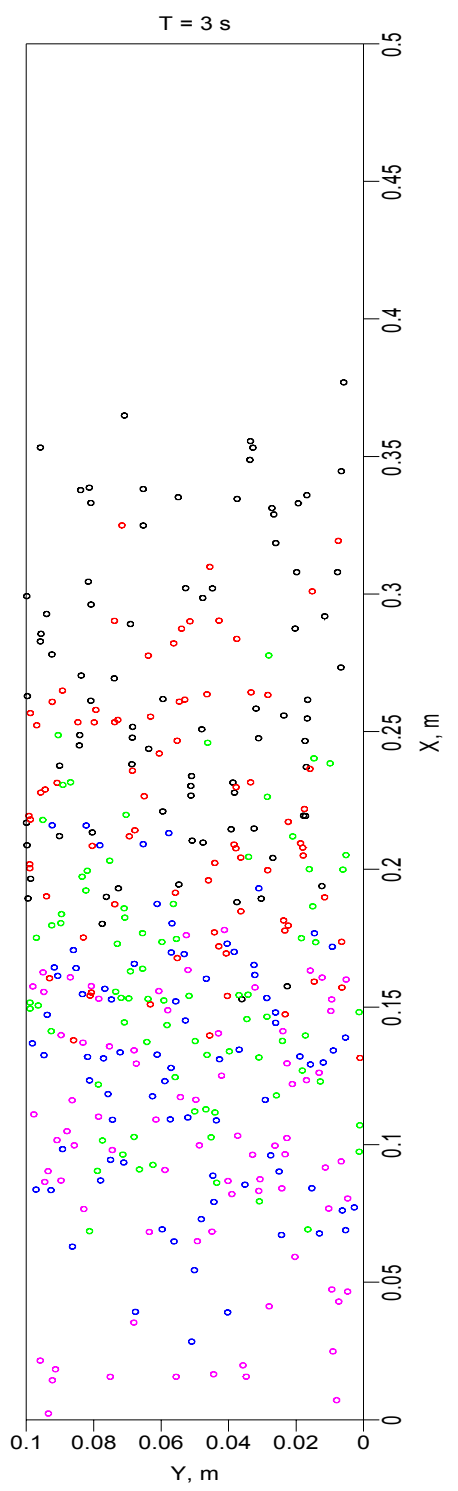

(b)

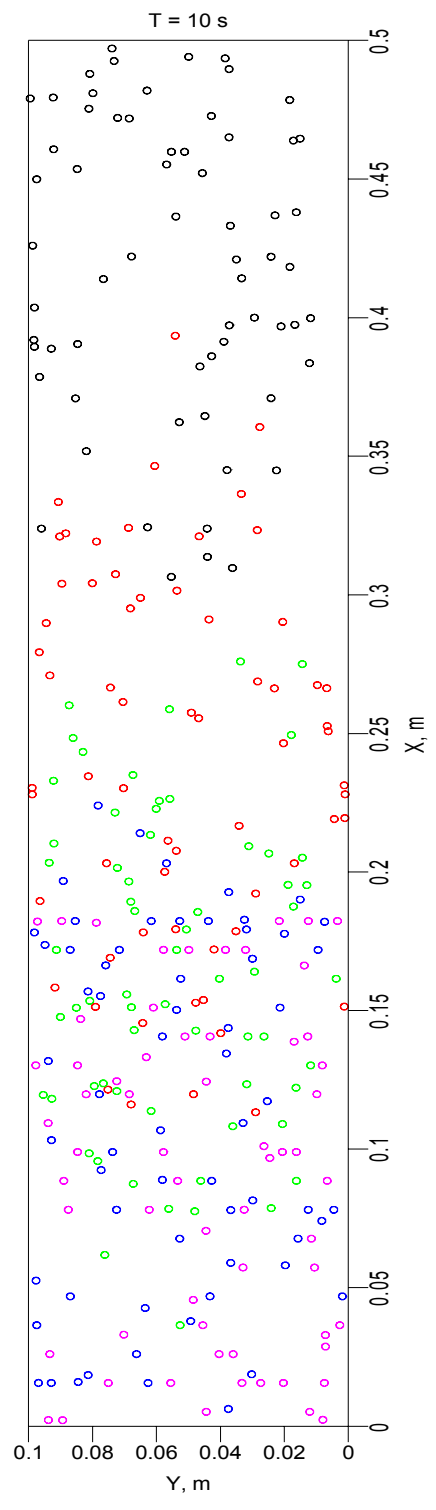

(c)

Particle Diamete

$0.0002 \mathrm{~m}$ to $0.0011 \mathrm{~m}$ $0.0011 \mathrm{~m}$ to $0.0021 \mathrm{~m}$ $0.0021 \mathrm{~m}$ to $0.0030 \mathrm{~m}$

$0.0030 \mathrm{~m}$ to $0.0040 \mathrm{~m}$

$0.0040 \mathrm{~m}$ to $0.0049 \mathrm{~m}$

Figure 6.13 Hindered-settling of particles with different sizes and a constant density 
In the second simulation case, the particle size is fixed at $2 \mathrm{~mm}$ and the particles have an evenly weight-percentage-distributed density composition, while keeping other parameters the same as those in first case. As shown in Figure 6.14, the light particles move upward and the heavy particles settle downward to channel bottom. Those particles with a medium density (specific density between 1.6 and 1.9) tend to accumulate in the middle of the channel. It is also noted from the simulation results that there is migration phenomena of the particles. The dense particles in an upward flow migrate towards the tube axis, while light spheres move towards the tube wall.

In the third simulation case, a particle body with a broad size distribution and density compositions are continuously added in the middle of channel while keeping the other parameters the same as above in previous second simulation case at a time $=3$ seconds. Figure 6.15(a) shows the particle distribution based on size and Figure 6.15(b) describes the effect of density. It is shown that the fine and light particles move upward while the heavy and coarse particles settle down. A particle with a medium size and density will accumulate in the middle of channel. If the size of a particle is too small, it will be carried upward even if it has a high density. On the other hand, a very coarse particle will move downward even if it has a low density. In the fluidization of the particles, the accumulated particles in the channel increase the suspension density which then forms a density gradient which helps the particle separation mainly based on density rather than on size. Thus, the formation of a fluidization bed inside the HSBS is critical to density separation. The fluidized-bed height and suspension density can be controlled by upward liquid flow and by feed properties. 

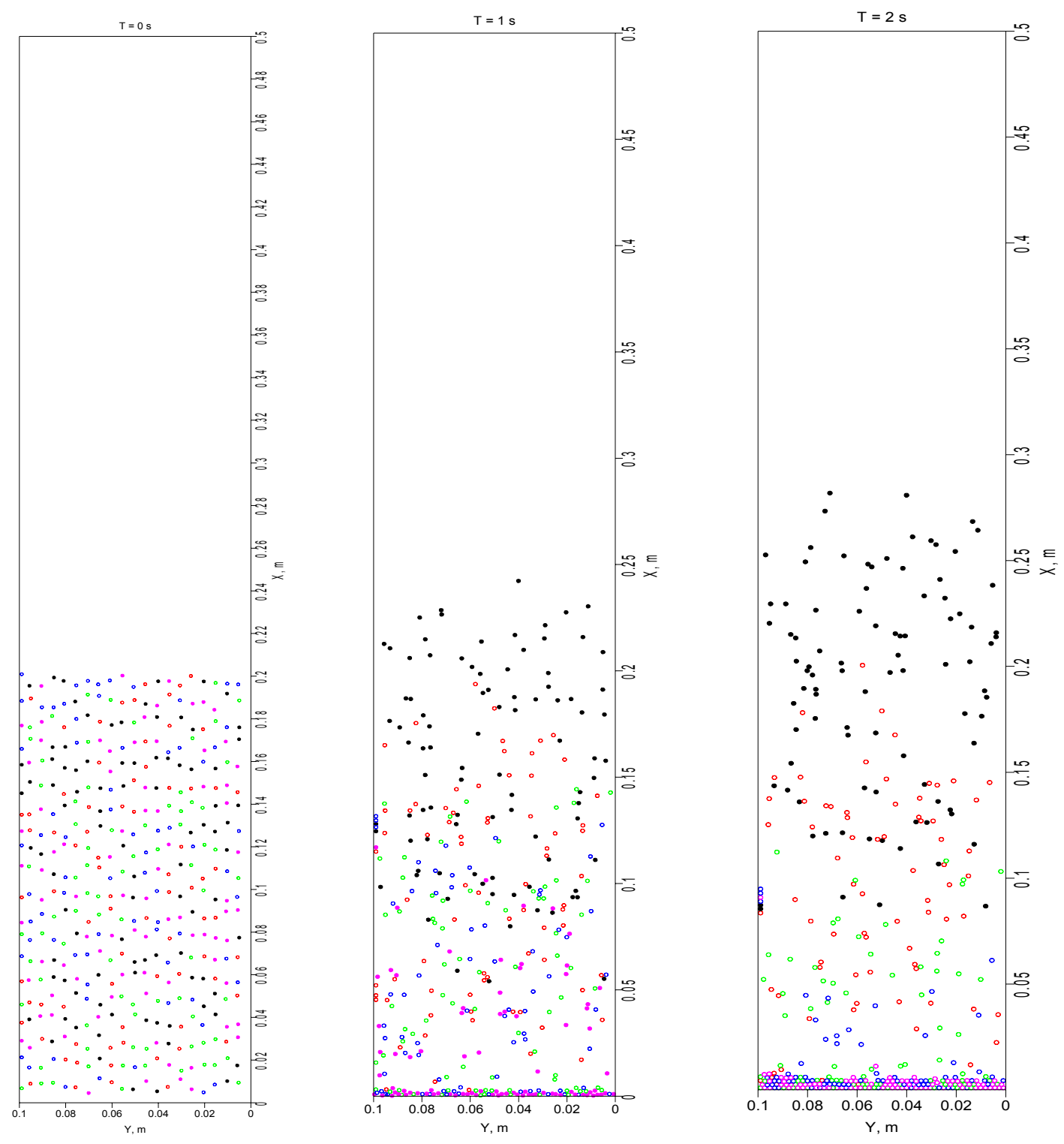

Figure 6.14 Hindered-settling of uniform sized particles with different densities 


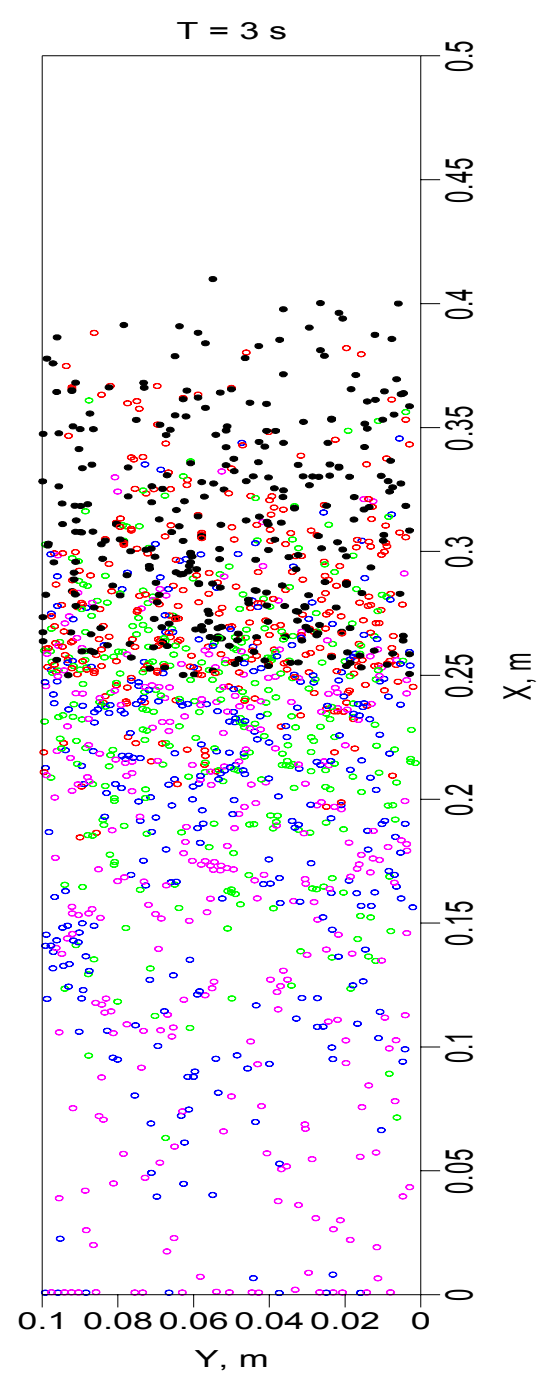

(a) size

Particle Diameter

$0.0002 \mathrm{~m}$ to $0.0011 \mathrm{~m}$ $0.0011 \mathrm{~m}$ to $0.0021 \mathrm{~m}$ $0.0021 \mathrm{~m}$ to $0.0030 \mathrm{~m}$

$0.0030 \mathrm{~m}$ to $0.0040 \mathrm{~m}$

$0.0040 \mathrm{~m}$ to $0.0049 \mathrm{~m}$

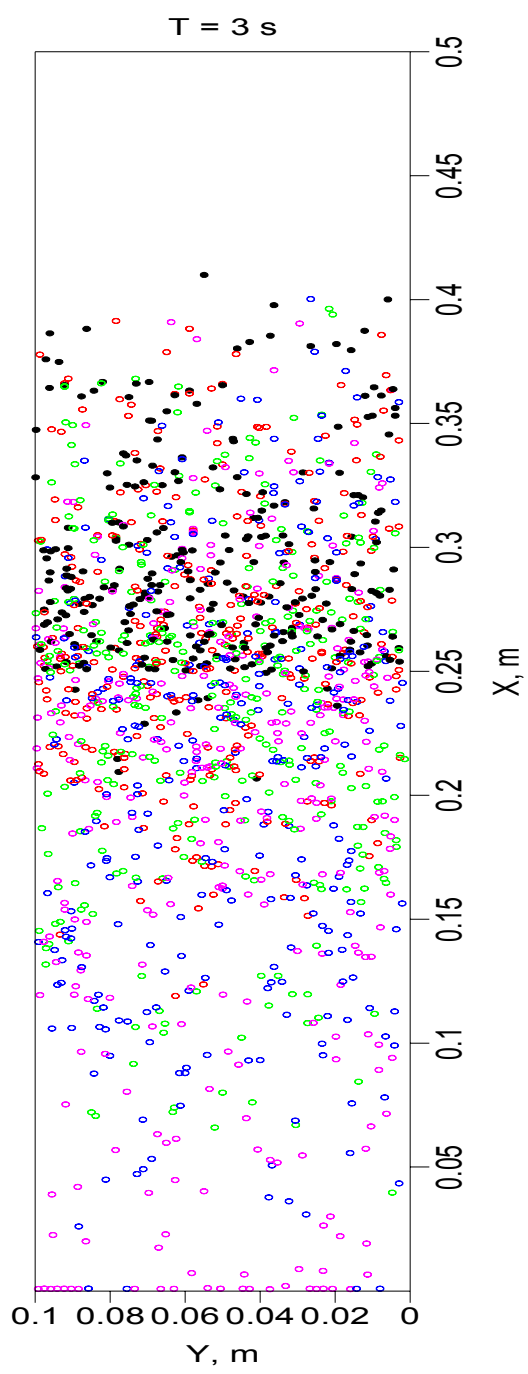

(b) density

Particle density

$1300 \mathrm{mg} / \mathrm{cm} 3$ to $1597 \mathrm{mg} / \mathrm{cm} 3$ $1597 \mathrm{mg} / \mathrm{cm} 3$ to $1894 \mathrm{mg} / \mathrm{cm} 3$ $1894 \mathrm{mg} / \mathrm{cm} 3$ to $2191 \mathrm{mg} / \mathrm{cm} 3$

$2191 \mathrm{mg} / \mathrm{cm} 3$ to $2488 \mathrm{mg} / \mathrm{cm} 3$

$2488 \mathrm{mg} / \mathrm{cm} 3$ to $2786 \mathrm{mg} / \mathrm{cm} 3$

Figure 6.15 Hindered-settling of particles with broad size distribution and density composition 


\subsection{Inversion in the Fluidized-Bed}

The effect of the fluidizing water velocity on the particle stratification is also examined. The phenomena of inversion of different particles were illustrated by Moritomi et al. (1982). In this phenomenon, a binary suspension of relatively fine dense particles and larger, less dense particles are separated or mixed at low, medium and high rate fluidization. At low fluidizing flow velocity, a high suspension density is produced by the fine heavy particles staying near the bottom of the bed. The density of the lower bed is high enough to support the coarse light particles. However, as the fluidizing water velocity increases, the fine particles are elutriated and thus decrease the fluidized-bed density. Thus, the suspension density is not high enough to support the coarse light particles. The coarse light particles begin to descend to the bottom of the bed and the two groups of particles are mixed. At high fluidizing water velocity, mixing disappears and the particle system is inverted with coarse particles sitting on the bottom of the bed. Many slip velocity models failed to predict this inversion phenomena. Here, we use Euler-Lagrange model to simulate this phenomena. We increase the fluidizing water velocity and keep other parameters constant. A binary mixture is

made of particles A and B. Group A particles have a size of $0.4 \mathrm{~mm}$ and specific density of 2.5. Group B particles have a size of $2 \mathrm{~mm}$ and a specific density of 1.30 . The size ratio of coarse particle to fine particle is 5.0. As shown in Figure 6.16(a), at low fluidizing water velocity $(\mathrm{u}=1 \mathrm{~mm} / \mathrm{s})$, the group of low density particles are accumulated near the bottom of channel and form a stable fluidized-bed with a specific suspension density about 1.5 which is high enough to support the group B particles. With the increase of fluidizing water velocity, the two groups of particles begin to be mixed as shown in Figure 16(b), and, eventually, at high fluidizing flow velocity, the coarse particles will settle down at the bottom of the 


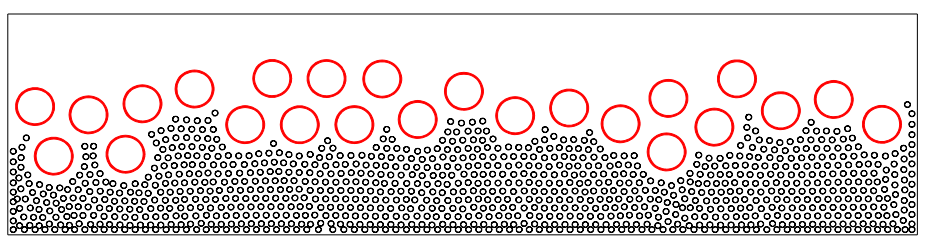

(a) $U=0.001 \mathrm{~m} / \mathrm{s}$

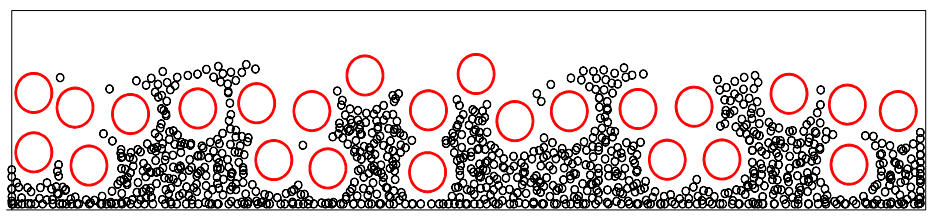

(b) $U=0.005 \mathrm{~m} / \mathrm{s}$

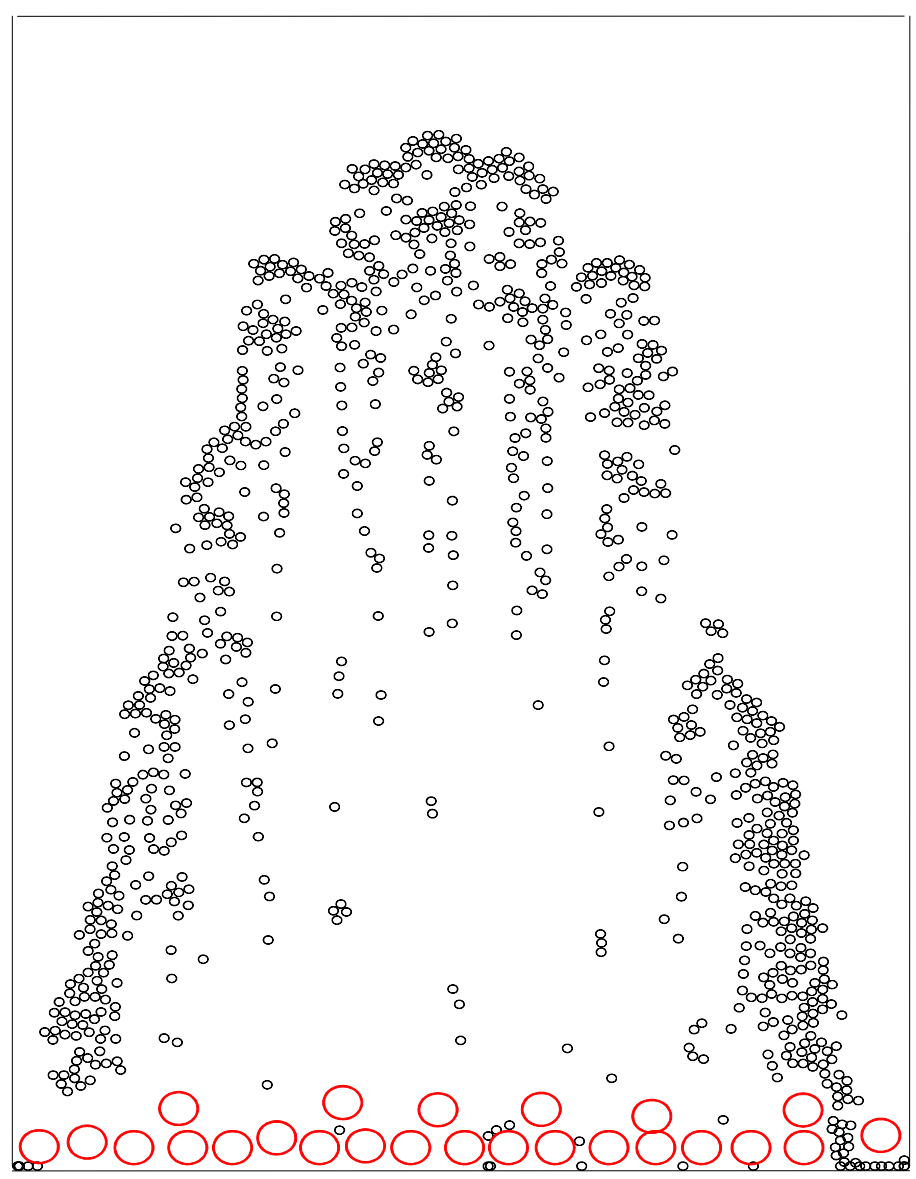

(c) $U=0.01 \mathrm{~m} / \mathrm{s}$

Figure 6.16 Effects of fluidizing water velocity on particle stratification 
channel while the fine particles are flushed away as shown in Figure 16(c).Thus, the stratification can be inverted by controlling fluidizing water velocity. From this simple simulation case, it is known that, in order to get a density based separation in a hinderedsettling bed, the fluidizing water velocity is one key parameter. The gravity separation of the particles is promoted by using relatively low fluidizing velocities and hence high suspension densities. By increasing the fluidizing water velocity, which in turn lowers the suspension density, a separation more dependent on the particle size is achieved, although particle density will continue to play a role.

\subsection{Summary}

- The behavior of both isolated particles and particle groups of fine coal in hinderedsettling bed separator was studied using the developed Euler-Lagrange model. The hindered-settling velocity is a function of particle size, particle density and suspension properties.

- The natural packing of uniform particle and multiple sized particles was also simulated in order to validate the collision model. All particles are separate well from wall and from each other.

- The Euler-Lagrange model can capture the basic characteristic properties of the jigging process.

- The model accurately describes coal particle motion and local liquid flow pattern changes in an initial static solid-liquid mixture. It also simulates the effect of particle size and density on particle separation as well as the fluidizing water velocity on the stratification inversion. 


\section{Chapter 7 Particle Density Separation in Hindered- settling Bed Separator}

The hindered-settling bed separator (HSBS) is designed for fine particle density separation. The solid-liquid granular flow was studied in a two-dimensional HSBS with a center feed system. The main focus of the investigation was to calculate the flow pattern in the separator and to assess the role of different operating parameters through simulation in order to obtain a better understanding of the separation mechanism. Performance data for the HSBS obtained in a coal preparation plant was used to validate the numerical simulation results for the main operating parameters on particle density separation. The simulation is based on the dynamical turbulent two-dimensional two-phase Euler-Lagrange model.

\subsection{Introduction}

The modeling of the hydrodynamics of the solid-liquid phase in a HSBS based on the continuity equation, Navier-Stokes Equations as well as particle movement equations was described and discussed in Chapters 3 to 5 . The feed used in the simulation has a broad size distribution and density compositions. The effect of various parameter values on the density particle separation will be considered and simulated.

\subsubsection{Hydrodynamic Model Basis}

The multiphase-flow hydrodynamic model used in this study is presented and discussed in Chapters 3 to 5 . The numerical simulation procedures are summarized below. The CFD model is a two-dimensional, two-phase, Euler-Lagrange model where the continuous liquid 
phase is represented through continuity and Navier-Stokes equations. The dispersed solid phase is calculated though tracking a number of discrete particles using the particle movement equations. Coupling between the solid and liquid phases are considered through updating liquid volume fractions and momentum transfer terms between the two phases. The model is solved by implicitly integrating in time using a finite volume discretization over space. The calculation of particle-particle and wall-particle collisions is also incorporated in each time step. The schematic diagram of hindered-settling bed separators used in the simulations is shown in Figure 7.1.

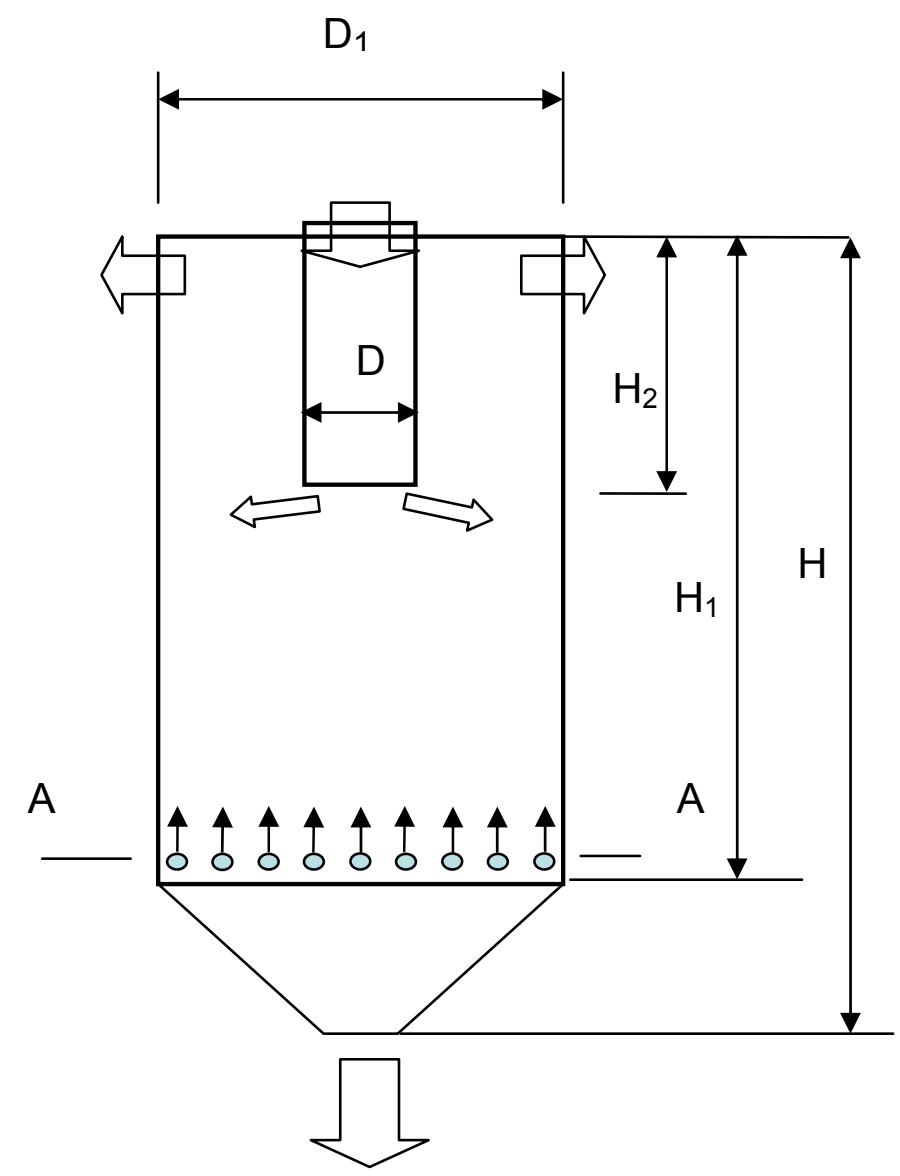

Figure 7.1 Schematic diagrams of hindered-settling bed separators with center tangential feed system 


\subsubsection{Density Separation for Fine Coal}

For density separation of fine coal, the goal is to obtain the particles with a density higher than the set point to report to the underflow stream, and particles of lower density to report to the overflow product stream. The overflow product should have a lower density and low ash content. The bottom reject composes of higher density particles with high ash content. In a HSBS, the upward current hinders the free settling of particles and forms a solid fluidizedbed which has a high suspension density that will help to govern the density separation. Only particles with high density can penetrate through this dense fluidized-bed and report to the reject stream at the bottom of the separator. The suspension density of the fluidized-bed can be controlled by the particle's properties including size, density and by the liquid phase properties including fluidizing water velocity and water viscosity. Under the same operation conditions, particles with different size distributions and density compositions will show different density separation efficiencies.

As shown in Figure 7.2, the density distribution curve shows the probability of particles with different relative density reporting to the clean coal product stream. To characterize this distribution curve a two-parameter logistic function as defined in Eq. (7-1) through model discrimination was developed by Klima and Luckie (1988)

$$
\mathrm{R}_{\mathrm{ji}}(\mathrm{x}, \mathrm{SG}) \%=\frac{100}{1+\exp \left[\left(1.098 / \mathrm{Ep}_{\mathrm{ji}}\right)\left(\mathrm{SG}-\mathrm{SG} 50_{\mathrm{ji}}\right)\right]}
$$

where $R_{j i}$ is the percent of feed material in the relative density interval of $S_{j}$ and of $S_{j+1}$ for the size interval of $x_{i}$ and $x_{i+1}$ reporting to the overflow product; $E p_{i}$ is the probable error for size $\mathrm{x}_{\mathrm{i}}$ and $\mathrm{x}_{\mathrm{i}}+\mathrm{dx} \mathrm{x}_{\mathrm{i}}$; and $\mathrm{SG} 50_{\mathrm{i}}$ is the specific gravity of separation. 


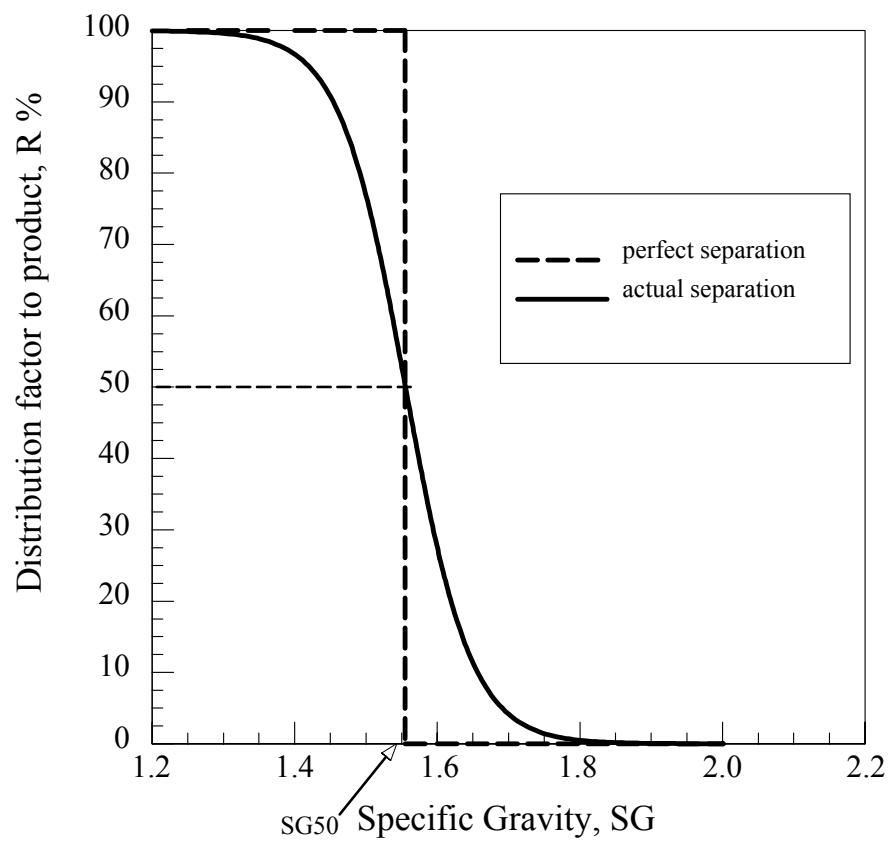

Figure 7.2 Distribution curve for density separation

The first parameter is the location modulus which has been termed the density of separation or density cut-point, SG50, as the specific gravity corresponding to $50 \%$ recovery on the distribution curve. At the SG50 specific gravity point, the particles have an equal probability of reporting to the product or refuse. The other parameter is the probable error defined as

$$
\mathrm{Ep}=\frac{1}{2}(\mathrm{SG} 25-\mathrm{SG} 75)
$$

where SG25 is the specific gravity corresponding to $25 \%$ probability on the distribution curve. SG75 is the specific gravity corresponding to $75 \%$ probability on the distribution curve. Ep gives a measure of the shape of the curve and the quality of separation. As Ep decreases, the curve approaches a vertical line passing through the SG50 shown as the dash line in Figure 
7.2. With this vertical curve, all particles having a density less than SG50 will report to the overflow product, the remaining materials reporting to the refuse and the separation is perfect. However, in actual separation, density separation tends to be affected by particle sizes. The fine particle having a high density tends to be mixed in overflow product because of mixing and elutriation by liquid flow. Usually a less than perfect separation will invariably result as shown as the solid line in Figure 7.2.

By using float-sink analysis of clean coal product, refuse and feed, the distribution curve for each size fraction can be determined and drawn. The parameters SG50 and Ep which characterize the distribution curve are obtained through data fitting of the model defined in Eq. (7-1) with a nonlinear optimization routine.

\subsection{In-Plant Performance Tests}

The coal sample used to validate the simulation model was taken from the HSBS in Century Mine Coal Preparation Plant, American Energy Inc., Alledonia, Ohio. For in-plant testing, the samples of feed, clean coal and refuse were taken every 15 minutes from the HSBS system (Stokes's TBS) for a period of 4 hours. Size distribution and float-sink test results for

feed, clean coal product and refuse are used in the analysis. The size distribution and washability analysis for fine coal feed sample (Pittsburgh \#8 coal, OH) are shown in Tables 7.1 and 7.2, respectively (Peng et al., 2004a). The feed has a maximum size of $5 \mathrm{~mm}$. The coal feed sample has $21.33 \%$ ash content, and consists of mostly $-2.38+0.3 \mathrm{~mm}$ size fraction. The feed contains nearly $10 \%$ of minus $150 \mu \mathrm{m}$ fine coal with $34.47 \%$ ash content. The particles coarser than $2 \mathrm{~mm}$ comprise approximately $15 \%$ of feed. 
Table 7.1 Size distribution of fine coal sample (Pittsburgh \#8 coal, OH)

\begin{tabular}{|r|r|r|r|r|r||}
\hline $\begin{array}{l}\text { Particle } \\
\text { fraction } \\
(\mathrm{mm})\end{array}$ & $\begin{array}{l}\text { Weight } \\
\text { Fraction } \\
(\%)\end{array}$ & $\begin{array}{l}\text { lum. Wt } \\
(\%)\end{array}$ & $\begin{array}{l}\text { Weight } \\
\text { Fraction } \\
(\%, \text { deslime })\end{array}$ & $\begin{array}{l}\text { Cum. Wt } \\
(\%, \text { deslime })\end{array}$ & $\begin{array}{l}\text { Individual Ash } \\
(\%)\end{array}$ \\
\hline+2.38 & 4.87 & 100.00 & 5.68 & 100.0 & 33.97 \\
\hline$-2.38+1.68$ & 11.27 & 95.13 & 13.15 & 94.32 & 17.33 \\
\hline$-1.68+1.18$ & 12.92 & 83.85 & 15.07 & 81.17 & 8.30 \\
\hline$-1.18+1.00$ & 6.09 & 70.93 & 7.11 & 66.10 & 21.84 \\
\hline$-1.00+0.85$ & 6.88 & 64.84 & 8.03 & 58.99 & 24.02 \\
\hline$-0.85+0.60$ & 14.51 & 57.96 & 16.93 & 50.96 & 16.71 \\
\hline$-0.60+0.425$ & 11.77 & 43.45 & 13.72 & 34.03 & 23.91 \\
\hline$-0.425+0.30$ & 11.43 & 31.69 & 13.34 & 20.31 & 24.59 \\
\hline$-0.30+0.212$ & 5.97 & 20.25 & 6.97 & 6.97 & 21.53 \\
\hline$-0.212+0.15$ & 4.35 & 14.28 & 0 & 0 & 20.94 \\
\hline-0.15 & 9.93 & 9.93 & 0 & 0 & 34.47 \\
\hline Total & 100.00 & & & & \\
\hline
\end{tabular}

Table 7.2 Washability analysis of fine coal sample (Pittsburgh \#8 seam coal, OH)

\begin{tabular}{|c|c|c|c|}
\hline $\begin{array}{l}\text { Specific } \\
\text { gravity } \\
\text { interval }\end{array}$ & $\begin{array}{l}\text { Individual } \\
\mathrm{Wt} \\
(\%)\end{array}$ & $\begin{array}{l}\text { Cum. Wt } \\
(\%)\end{array}$ & $\begin{array}{l}\text { Cum. } \\
\text { Ash } \\
(\%)\end{array}$ \\
\hline$<1.3$ & 41.19 & 41.19 & 5.66 \\
\hline $1.3-1.4$ & 21.19 & 62.38 & 6.32 \\
\hline 1.4-1.6 & 16.28 & 78.66 & 7.89 \\
\hline $1.6-1.8$ & 3.92 & 82.58 & 8.63 \\
\hline $1.8-2.0$ & 1.82 & 84.40 & 9.37 \\
\hline $2.0-2.2$ & 2.87 & 87.27 & 10.73 \\
\hline $2.2-2.45$ & 3.34 & 90.61 & 13.04 \\
\hline $2.45-2.8$ & 9.39 & 100.00 & 19.27 \\
\hline
\end{tabular}




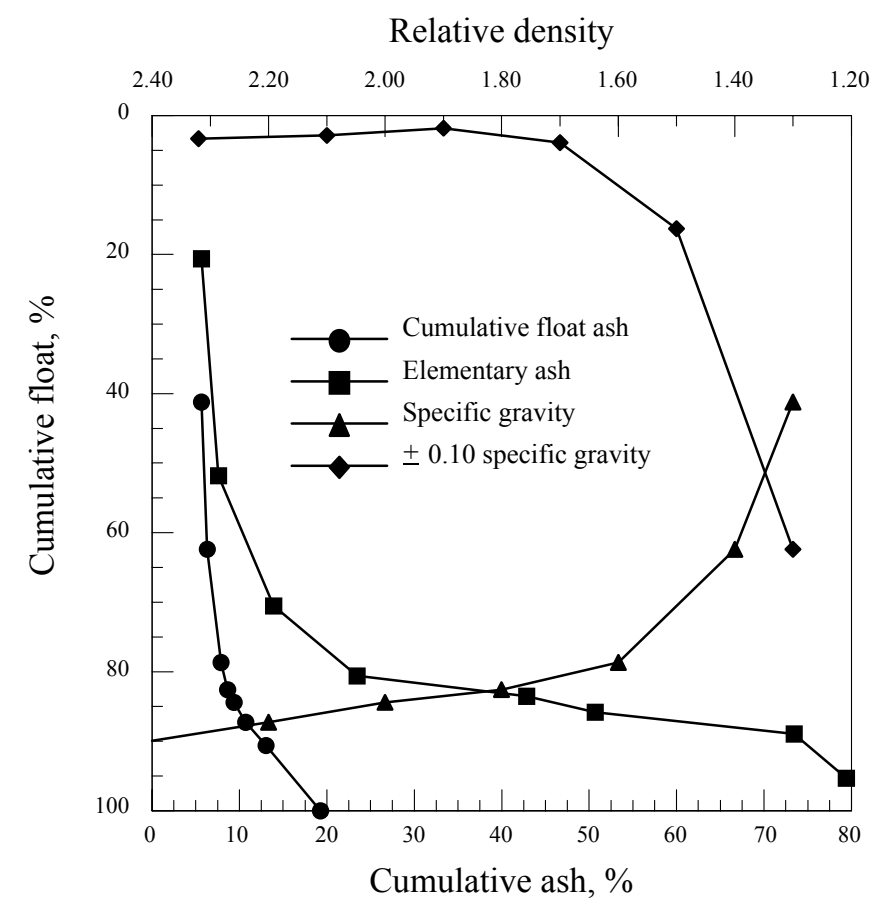

Figure 7.3 Washability of fine coal feed

From Table 7.2, the particles with a relative density lower than 1.4 consist of $62.38 \%$ of the feed, and average $6.32 \%$ ash content. The schematic diagram of the washability curves based on the float-sink tests is shown in Figure 7.3. The feed shows an easy-to-clean washability. The feed contains only $16.28 \%$ of middle-density particles (specific gravity $1.4-$ 1.6).

\subsection{Simulation of Feeding Materials}

The particles used in the simulations are randomly generated by computer. The size, density and other properties can be defined for each discrete particle from calculations based 
on the measurements. For a fixed density interval, the probability of a particle to be produced for a predefined size is shown in Eq. (7-3):

$$
\mathrm{P}_{\mathrm{si}}=\frac{\frac{\mathrm{W}_{\mathrm{si}}}{\mathrm{r}_{\mathrm{i}}^{3}}}{\sum_{\mathrm{i}=1}^{\mathrm{i}=\mathrm{n}_{1}} \frac{\mathrm{W}_{\mathrm{si}}}{\mathrm{r}_{\mathrm{i}}^{3}}}
$$

where $\mathrm{W}_{\mathrm{si}}$ is the measured weight percent of feed material for size interval of $r_{i}$ and $r_{i+1}$; $P_{s i}$ is the probability of a particle to be produced with a size in interval of $r_{i}$ and $r_{i+1}$; and $n_{1}$ is the total size intervals in feed.

For a fixed size interval, the probability of a particle to be produced with a predefined density is described in Eq. (7-4)

$$
P_{d i}=\frac{\frac{W_{d i}}{\rho_{i}}}{\sum_{i=1}^{i=n_{2}} \frac{W_{d i}}{\rho_{i}}}
$$

where $W_{d i}$ is the measured weight percent of feed material in relative density interval of $\rho_{j}$ and of $\rho_{\mathrm{j}+1} ; \mathrm{P}_{\mathrm{dj}}$ is the probability of a particle to be produced for density between $\rho_{\mathrm{j}}$ and $\rho_{\mathrm{j}+1}$ in feed, and $\mathrm{n}_{2}$ is total density fractions in feed.

In this simulation, it is assumed that density compositions are independent of the particle size intervals. If a randomly produced probability is within two neighboring cumulative calculated probabilities, then the particle will take a random value within the corresponding size and density fraction ranges. The randomly generated distribution data is compared with the measured cumulative weight percentage values. The statistical averaged size and relative density cumulative distributions of 2000 randomly generated fine coal particles are compared with the measured values in Figures 7.4 and 7.5. The results show that, although each discrete particle takes a value randomly, the overall statistical averaged properties for all 


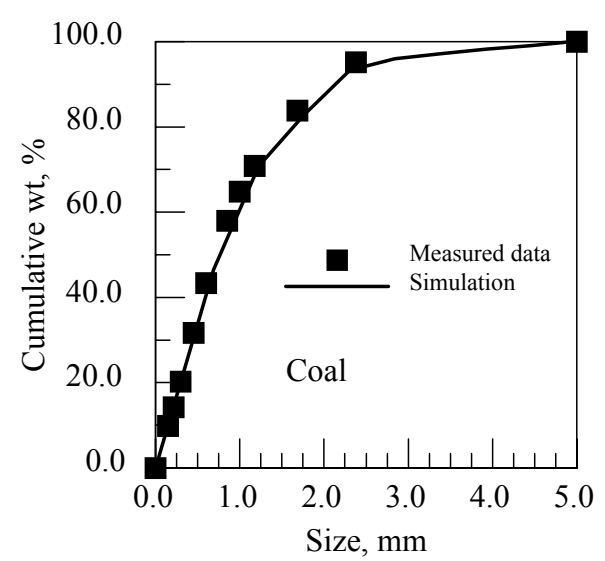

Figure 7.4 Cumulative $w t \%$ as a function of particle size for fine coal feed

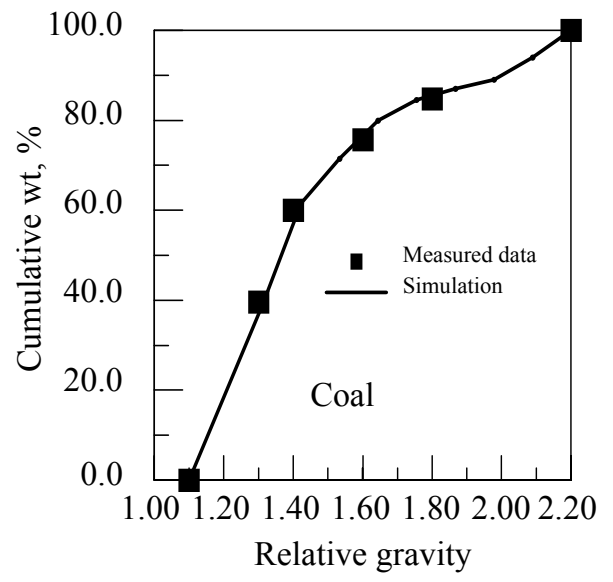

Figure 7.5 Cumulative wt $\%$ as a function of relative density for fine coal feed

particles agree well with the measured values.

\subsection{Simulation Results and Discussions}

The dimensions and parameters of the unit operations used in this simulation of separation are shown in Table 7.3 . The water density of $1.0 \mathrm{~g} / \mathrm{m}^{3}$ and kinetic viscosity of $0.01 \mathrm{~m}^{2} / \mathrm{s}$ are used in simulation work. For computational parameters, the time step is $0.005 \mathrm{~s}$ and the numbers of computational cells are $50 \times 50$. The simulations for a HSBS are listed in Table 7.3 with operation conditions and equipment dimensions defined in detail.

In case 1 , the feed has a uniform size of $1 \mathrm{~mm}$. The entire feed is composed of particles with a specific density of 1.2 to 2.8 . The purpose of this case is to show a typical liquid flow pattern and particle movement in the HSBS. The computed flow field is described by liquid 
contours and vector maps. The particle density distribution, suspension density and particle Reynolds number can also be determined through integration of discrete particle movement.

In case 2 to case 6 , the effects on the density separation by different operating parameters are investigated. The changes in SG50 and Ep values are compared for each parameter at different levels.

In case 2, the size effect is determined. The feed has a fixed density composition and a specific set of operating conditions. The distribution curves for five size fractions are compared. These size fractions are $-2+1.4 \mathrm{~mm},-1.4+1.0 \mathrm{~mm},-1.0+0.7 \mathrm{~mm},-0.7+0.5 \mathrm{~mm}$ and $-0.5+0.25 \mathrm{~mm}$. This simulation provides a basis for determining the size effect on the separation performance. Normally the HSBS is operated with a feed size similar to the one used in this case.

In case 3 , the feed particle size is fixed at $-1.0+0.7 \mathrm{~mm}$. The solid feed velocity and solid concentration are the same as case 1, but the fluidizing water velocity is changed. It is noted that fluidizing flow velocity tends to increase the SG50 because of an increasing elutriation effect.

Case 4 is the same as case 3 , but at different set points for suspension density rather than at different fluidizing water velocities. The suspension density can be controlled by the under flow discharge rate. Five set-point suspension density levels are used. The aim is to demonstrate the fact that the suspension density value is one of the most critical parameters in determination of the density separation performance.

In case 5, the effect on the density separation from changes of geometry of the vessel is investigated. The operation conditions are the same as those in case 4 except at a constant specific set-point suspension density of 1.14 . This 1.14 set-point value is used in the actual 
Table 7.3 Parameters used in simulations

\begin{tabular}{|c|c|c|c|c|c|}
\hline \multicolumn{2}{|l|}{ Case } & 1 & 2 & 3 & 4 \\
\hline \multirow[t]{5}{*}{ Unit dimensions, $\mathrm{m}$} & $\mathrm{D}_{1}$ & 3.66 & 3.66 & 3.66 & 3.66 \\
\hline & $\mathrm{D}_{2}$ & 0.86 & 0.86 & 0.86 & 0.86 \\
\hline & $\mathrm{H}$ & 3.48 & 3.48 & 3.48 & 3.48 \\
\hline & H1 & 3.0 & 3.0 & 3.0 & 3.0 \\
\hline & $\mathrm{H} 2$ & 0.89 & 0.89 & 0.89 & 0.89 \\
\hline \multicolumn{2}{|l|}{ Coal size, $\mathrm{mm}$} & 1.0 & $0.25-2.0$ & $0.7-1.0$ & $0.7-1.0$ \\
\hline \multicolumn{2}{|l|}{ Coal density, $\mathrm{g} / \mathrm{cm}^{3}$} & $1.2-2.8$ & $1.2-2.8$ & $1.2-2.8$ & $1.2-2.8$ \\
\hline \multicolumn{2}{|c|}{$\begin{array}{l}\text { Fluidizing water velocity, } \\
\mathrm{m}^{3} / \mathrm{m}^{2} \mathrm{~h} \\
(\text { superficial fluidizing water } \\
\text { velocity, } \mathrm{mm} / \mathrm{s})\end{array}$} & $\begin{array}{l}24.12 \\
(6.7)\end{array}$ & $\begin{array}{l}32.4 \\
(9)\end{array}$ & $\begin{array}{l}19.44- \\
45.36 \\
(5.4- \\
12.6) \\
\end{array}$ & $\begin{array}{l}32.4 \\
(9)\end{array}$ \\
\hline \multicolumn{2}{|c|}{ Solid feed flow, $\mathrm{t} / \mathrm{m}^{2} \mathrm{~h}$} & 2.65 & 2.65 & 2.65 & 2.65 \\
\hline \multicolumn{2}{|c|}{ Feed solid concentration, $\%$} & 15 & 15 & 15 & 15 \\
\hline \multicolumn{2}{|c|}{$\begin{array}{l}\text { Water flow velocity in feeding } \\
\text { pipe }, \mathrm{m}^{3} / \mathrm{m}^{2} \mathrm{~h} \\
(\text { water velocity in feed pipe, } \\
\mathrm{mm} / \mathrm{s})\end{array}$} & $\begin{array}{l}75 \\
(130)\end{array}$ & $\begin{array}{l}15 \\
(26)\end{array}$ & $\begin{array}{l}15 \\
(26)\end{array}$ & $\begin{array}{l}15 \\
(26)\end{array}$ \\
\hline \multicolumn{2}{|c|}{ Set point relative density, $\mathrm{g} / \mathrm{cm}^{3}$} & 1.14 & 1.14 & 1.14 & \begin{tabular}{|l|}
$1.05-$ \\
1.20 \\
\end{tabular} \\
\hline \multicolumn{2}{|l|}{ Case } & 5 & 6 & 7 & 8 \\
\hline \multirow[t]{5}{*}{ Unit dimensions, $\mathrm{m}$} & $\mathrm{D}_{1}$ & - & 3.66 & 3.66 & 3.66 \\
\hline & $D_{2}$ & - & 0.86 & 0.86 & 0.86 \\
\hline & $\mathrm{H}$ & 3.48 & 3.48 & 3.48 & 3.48 \\
\hline & H1 & - & 3.0 & 3.0 & 3.0 \\
\hline & $\mathrm{H} 2$ & - & 0.89 & 0.89 & 0.89 \\
\hline \multicolumn{2}{|l|}{ Coal size, $\mathrm{mm}$} & $0.7-1.0$ & $0.7-1.0$ & $0.7-1.0$ & $0.2-2.0$ \\
\hline \multicolumn{2}{|c|}{ Coal density, $\mathrm{g} / \mathrm{cm}^{3}$} & $1.2-2.8$ & $1.2-2.8$ & $1.2-2.8$ & $1.2-2.8$ \\
\hline \multicolumn{2}{|c|}{$\begin{array}{l}\text { Fluidizing water velocity, } \\
\mathrm{m}^{3} / \mathrm{m}^{2} \mathrm{~h} \\
(\text { superficial fluidizing water } \\
\text { velocity, } \mathrm{mm} / \mathrm{s})\end{array}$} & $\begin{array}{l}32.4 \\
(9)\end{array}$ & $\begin{array}{l}32.4 \\
(9)\end{array}$ & $\begin{array}{l}32.4 \\
(9)\end{array}$ & \begin{tabular}{|l|}
24.12 \\
$(6.7)$
\end{tabular} \\
\hline \multicolumn{2}{|c|}{ Solid feed flow, $\mathrm{t} / \mathrm{m}^{2} \mathrm{~h}$} & 2.65 & $\begin{array}{l}2.65- \\
13.25\end{array}$ & $\begin{array}{l}2.65- \\
13.25\end{array}$ & 21.44 \\
\hline \multicolumn{2}{|c|}{ Feed solid concentration, $\%$} & 15 & 15 & $15-45$ & 45 \\
\hline \multicolumn{2}{|c|}{$\begin{array}{l}\text { Water flow velocity in feeding } \\
\text { pipe }, \mathrm{m}^{3} / \mathrm{m}^{2} \mathrm{~h} \\
(\text { water velocity in feed pipe, } \\
\mathrm{mm} / \mathrm{s})\end{array}$} & $\begin{array}{l}15 \\
(26)\end{array}$ & $\begin{array}{l}15-75 \\
(26-130)\end{array}$ & $\begin{array}{l}15 \\
(26)\end{array}$ & $\begin{array}{l}26.2 \\
(45)\end{array}$ \\
\hline \multicolumn{2}{|c|}{ Set point relative density, $\mathrm{g} / \mathrm{cm}^{3}$} & 1.14 & 1.14 & 1.14 & 1.14 \\
\hline
\end{tabular}


operation of the HSBS in the plant. The changes of geometric parameters of width/height of column can result in the changes of liquid flow and further affect the movement of the particles.

In case 6 , the slurry is introduced at different speeds at a constant solid concentration of $15 \%$. The other operating parameters are same as those in case $2,3,4$ and 5.

In case 7 , the fluidizing water velocity and water flow velocity in feed pipe are kept constant. The solid feed is introduced at different solid concentrations. The other operation conditions are the same as those defined in case 4.

Case 8 shows the simulation results and performance test results in-plant. The input data for the simulation model includes particle properties and operation conditions. The output results include the information on liquid flow pattern, particle distribution and statistical calculation of particle assembly.

\subsubsection{Flow Patterns}

The HSBS is characterized by simple construction and a complex flow structure. In this simulation, the fine coal is processed in the HSBS with a center feeding system. In case 1, the results of the continuous liquid velocity contours and vector maps of these unit operations are shown in Figure 7.6. The slurry is introduced through the feed pipe tangentially into the upper one-third of the unit. The feed particles form a fluidized-bed as they settle against an upward counter-current flow of fluidizing water. The feed can be introduced as dry material

or slurry. In case 1, the feed flow velocity is $24.12 \mathrm{~m}^{3} / \mathrm{m}^{2} \mathrm{~s}$ and the superficial fluidizing water velocity is $6.7 \mathrm{~mm} / \mathrm{s}$, and solid concentration in the feed is $15 \%$. There are two water sources going into the units. They are the fluidizing water and the water stream from the feed 
slurry. The water from the feed inlet is forced to flow upward to the overflow exit of the unit as illustrated in the flow directions of the water in Figure 7.6. This phenomenon can cause a disturbance in the fluidizing water field right below the feed inlet, depending upon the magnitude of the water stream and the relative height of the inlet pipe location. These two water streams merge near the feed inlet and flow to the overflow. Both water streams have no effect on the liquid flow in the dewatering zone, which is near the lower part of the unit and close to underflow exit. The liquid is nearly static in this region. In actual operation, the outgoing underflow has a high solid concentration, as high as $50 \%-80 \%$. Therefore, the liquid flow is nearly negligible in the dewatering zone, compared with much higher overflow water volume. As shown in Figure 7.6, the fluidizing water velocity is high at the location of injection points but decreases sharply after a short distance above the water distributor.

As shown in Figure 7.7 and 7.8 , the liquid phase velocity profiles for axial and radial components at different heights, $\mathrm{h}$, above A-A level in Figure 7.1 are compared respectively. In a fluidized-bed, the water velocity becomes constant above distributors up to the feed inlet. The maximum velocity is developed along the centerline of the column with no-slip conditions at the walls. At the center of the separator, the injecting water from feed pipe will cause a downward flow and its magnitude decreases with depth. This is the reason that tangential or radial spreading feed is critical to the separation performance. Above the feed inlet, however, the axial fluidizing water velocity outside the inlet pipe is increased by the additional water from the feed. The axial fluidizing water velocity is important, since it will determine the cutting size and specific gravity of separation (cut-points). The particles are stratified in the fluidized-bed and form a pulp gradient density. 


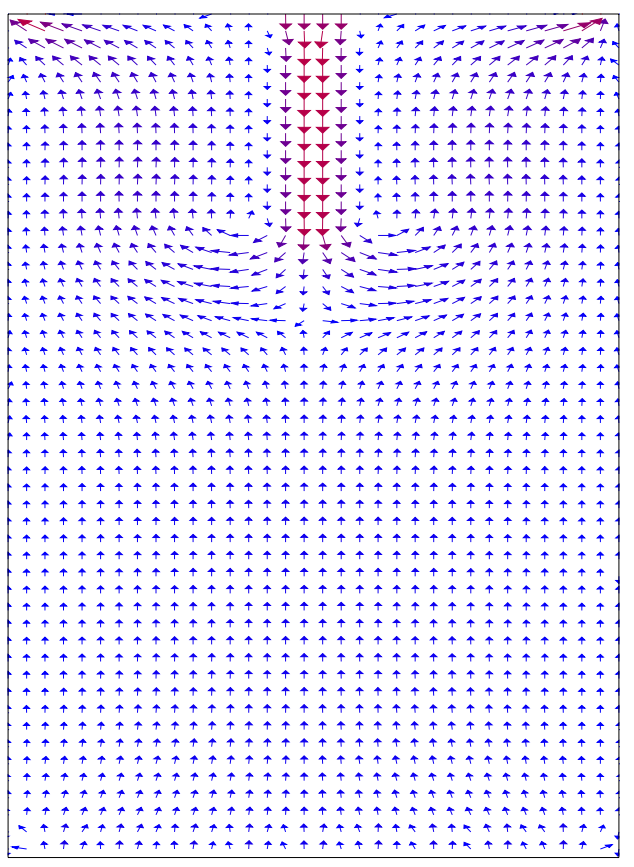

Figure 7.6 Liquid velocity field in hindered-settling bed separator with center-tangential feeding system

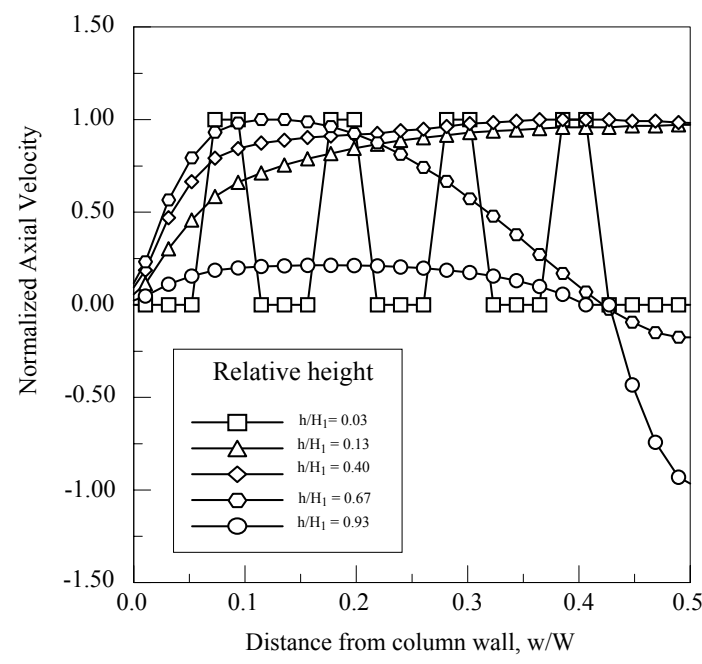

Figure 7.7 Normalized axial velocity for liquid phase at different heights

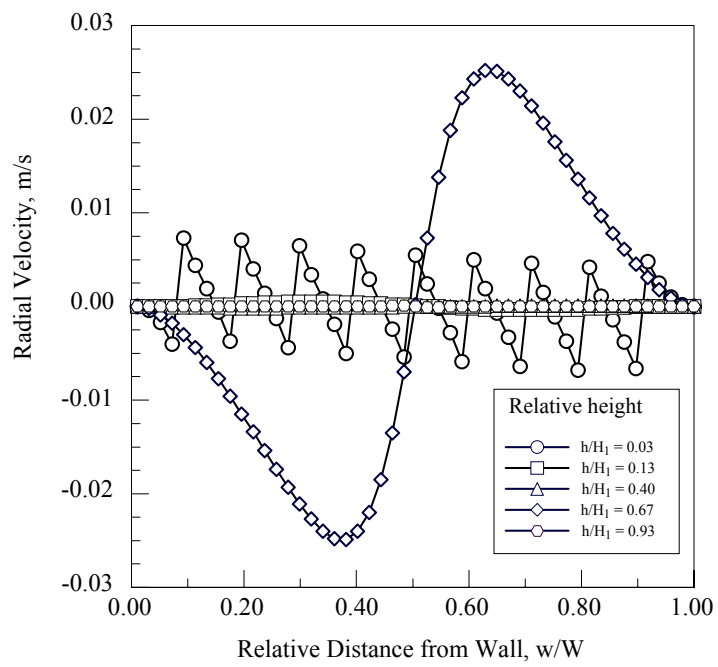

Figure 7.8 Radial velocity for liquid phase at different heights 
The particle Reynolds number distribution is described in Figure 7.9. It is shown that near the bottom of column, particles tend to have higher Reynolds numbers; this corresponds to the high liquid velocity at fluidizing water injecting points.

As shown in Figures 7.10(a) and 7.10(b), when particles have a uniform size of 1mm, at a superficial fluidizing water flow velocity of $6.7 \mathrm{~mm} / \mathrm{s}$, particles are separated based on density differences. The case 1 density separation has a density cut-point, SG50, at 1.7 and Ep at 0.07 .
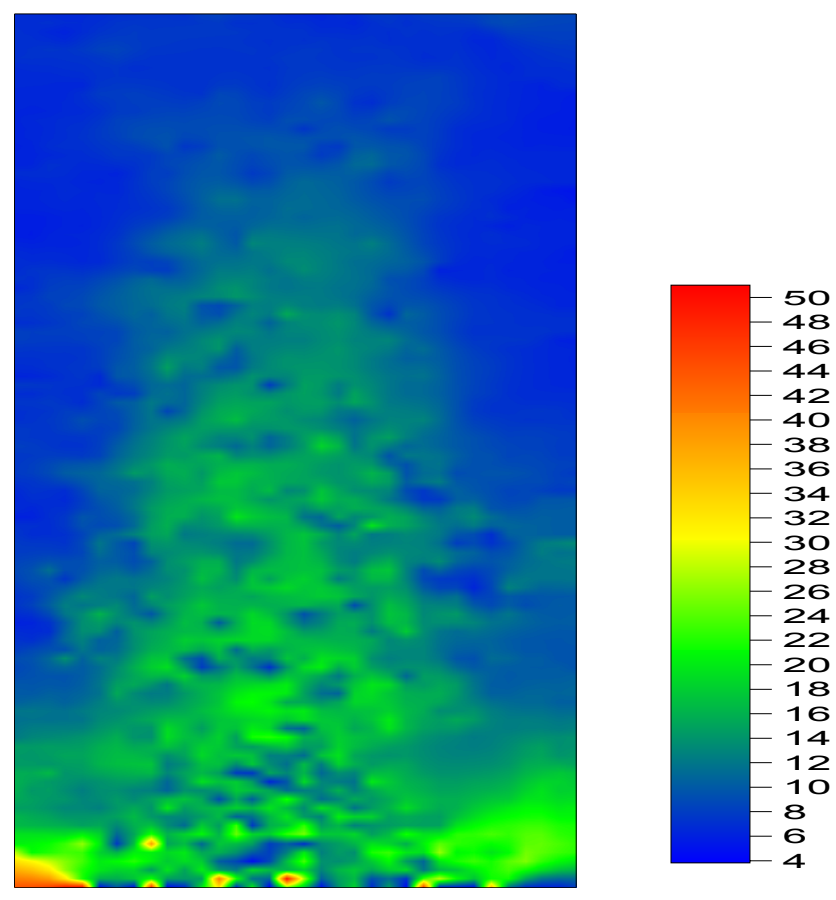

Figure 7.9 Particle Reynolds number distribution in hindered-settling bed separator 

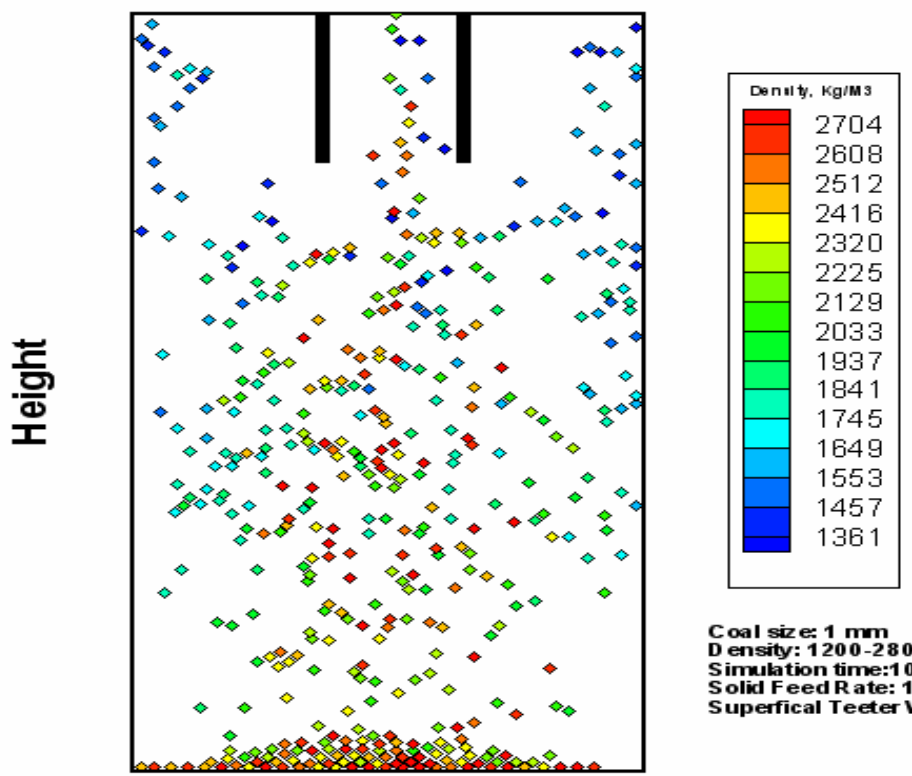

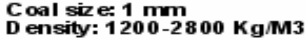

Simulation time: $100 \mathrm{~s}$

Solid Feed $R$ ate: 10 parcel/second

Superfical Teeter $W$ ater $F$ low $R$ ate: $0.007 \mathrm{~m} / \mathrm{s}$

Width

(a) Particle distribution

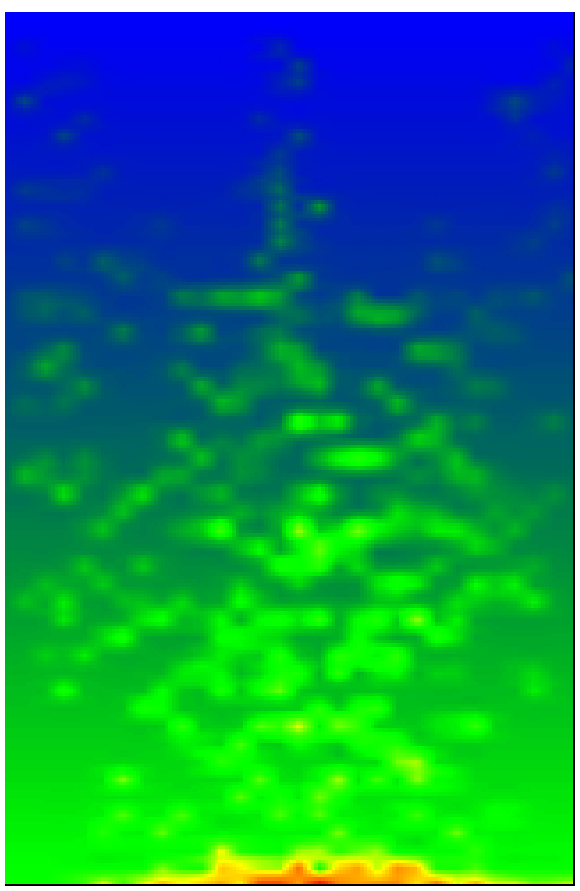

Density: $\mathrm{Kg} / \mathrm{M}^{3}$

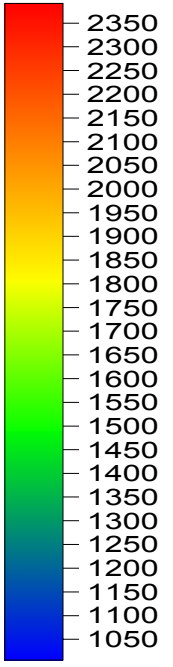

(b) Density distribution contour

Figure 7.10 Simulated particle distribution in hindered-settling bed separator 


\subsubsection{Operating Parameters of HSBS}

\subsubsection{Particle Size}

In case 2, the size effect was simulated with five particle size fractions at the same operation conditions and at same predefined simulation time as shown in Table 7.3. The simulated distribution curves as a function of particle size obtained from the washability analysis of the feed, product and reject streams for the five size fractions are shown in Figure 7.11. It is shown that the distribution curve will shift to a higher density as the particle size decreases. The density cut-point, SG50, increases from 1.3197, 1.4081, 1.5615, and 1.8345 to 2.3684 with corresponding separation efficiency, Ep, increases from 0.0146, 0.0202, 0.0542, and 0.0747 to 0.2112 . From the analysis of forces to which a particle is exposed to in Chapter 3 , it is known that decreasing the particle size will cause relatively higher drag force per unit particle mass, thus small particle will have a higher probability to report to the overflow than coarse particles.

In general, the separation achieved on the coarsest particles size is projected to yield the lowest SG50 and Ep values. Effective separations are predicted to a particle size ratio of about $3: 1$.

From the above results, it is expected that the narrower the feed size range, the better the separation performance. As the particle size of heavy particles in the feed increases relatively to that of light particles, the separation efficiency becomes better. When the particle size of the heavy particle in the feed is larger than that of the light particle, the particle size will have positive effect on this separation performance. On the other hand, if the particle size of light particles is larger than that of heavy particles, then there will be a negative effect on the separation with misplacement of coarse light particles in the underflow or fine heavy 
particles to the overflow. Thus, the preparation of the feed to different size ranges and processing separately as to size is a good approach to give better separation efficiency.

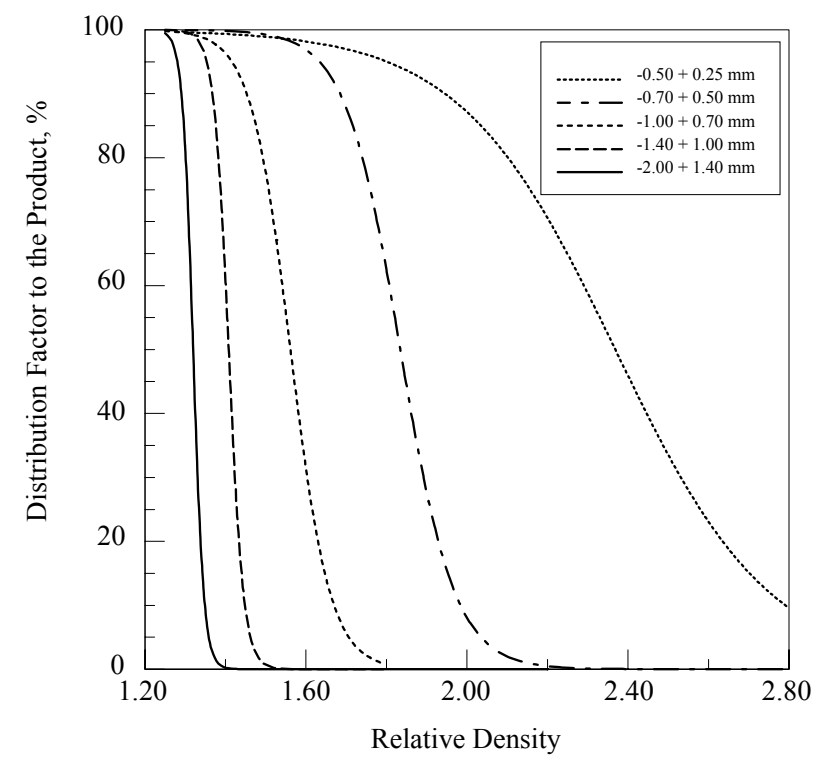

Figure 7.11 Effect of particle size on separation performance

\subsubsection{Fluidizing Water Velocity}

The operating variables play an important role in particle separation in a HSBS. The fluidizing water velocity is the most important operating parameter. Increasing the fluidizing water velocity increases the relative velocity between particles and the surrounding liquid, and results in a higher drag force, which tends to overcome the gravity force and drag particles upward. A higher fluidizing water velocity promotes a higher specific gravity of separation, SG50. On the contrary, lower fluidizing water velocity reduces SG50. Figure 7.12 illustrates the distribution curves for different fluidizing flow velocities for case 3 operation 
conditions for a size fraction of $-1.0+0.7 \mathrm{~mm}$. As expected, increasing the fluidizing water velocity also shifts the specific gravity of separation to a higher cut-point. At constant feed and superficial fluidizing water velocities of $5.4,7.2,9$ and $12.6 \mathrm{~mm} / \mathrm{s}$, the corresponding specific gravity of separations (SG50s) are 1.3765, 1.4711, 1.5615 and 1.7311 with the values of Ep at $0.0236,0.0434,0.0542$ and 0.0660 , respectively. Thus a lower fluidizing water velocity will result in a lower, sharper density separation.

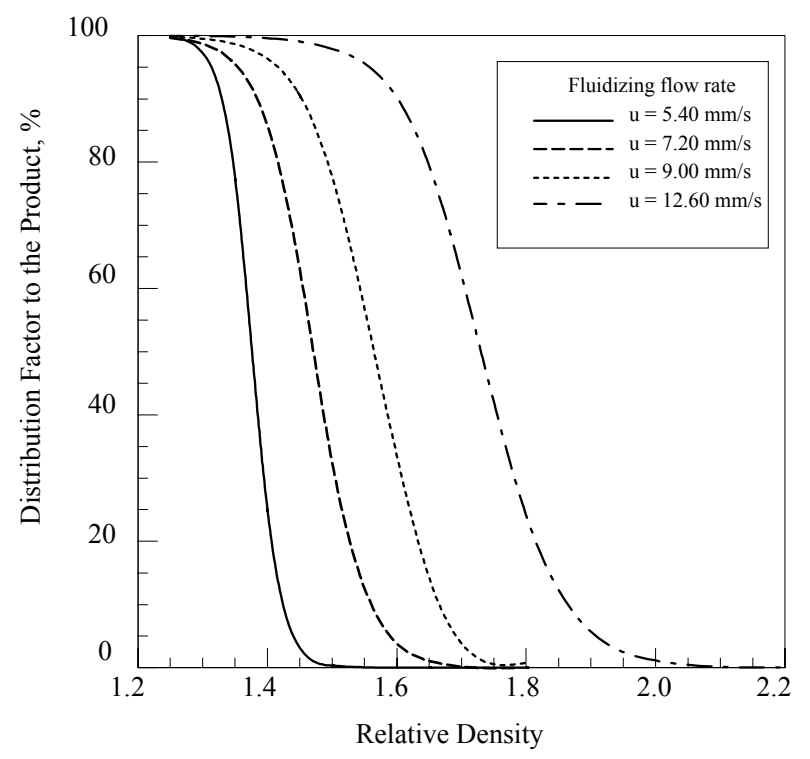

Figure 7.12 Effect of fluidizing water velocities on separation performance for size fraction of $-1.0+0.7 \mathrm{~mm}$

\subsubsection{Density Set Point}

The suspension density can be increased or decreased to adjust the separation. Increasing the density set-point will increase the size separation cut-point or specific gravity separation cut-point. In the HSBS, where the discharge of underflow stream is restricted; the heavier particles will accumulate in the dewatering zone and form a liquid-solid fluidized-bed above 
the fluidizing water inlet level. This bed of slurry will act as a dense-medium so that downward moving particles experience a density gradient different from pure liquid. The HSBS with the suspension density controlled is used for separations based primarily on particle density. This has been achieved by controlling the underflow removal rates. The density set-point at a fixed bed level can be adjusted by a pressure or density sensor, which is in combination with the actuator to operate the discharge valve. In case 4, the effect of the density set-point for a size fraction of $-1.0+0.7 \mathrm{~mm}$ is simulated and the results are shown in Figure 7.13. As expected, increasing the density set-point shifts the specific gravity of separation to a higher cut-point. At constant feed and superficial fluidizing water velocity of $9 \mathrm{~mm} / \mathrm{s}$, the corresponding specific gravity of separations, SG50s are 1.4188, 1.4319, 1.5007, 1.5615 and 1.5737 and the values of Ep at $0.0237,0.0455,0.0629,0.0542$ and 0.027 for a set point relative suspension density values of $1.05,1.10,1.15,1.20$ and 1.25 respectively. The increase of density cut-point can be attributed to the generation of the denser bed, and to a steep density gradient that built along the column height, and hence, a higher buoyant force acting on the particles in a denser fluidized-bed.

\subsubsection{Geometry of Column}

Figure 7.14 shows the effect of column width/height ratios on density separation in case 5. The separator height is constant, but the width of column is varied to investigate the separation performances. Overall, the density separation performances are about the same if width/height ratio is larger than 0.15 . The separation deteriorates with decreasing 


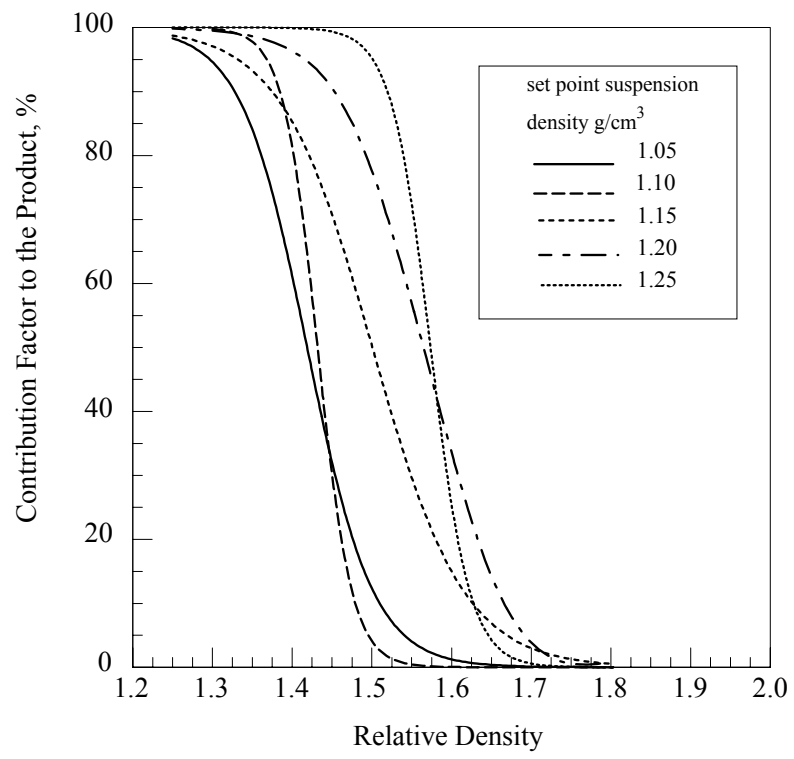

Figure 7.13 Effect of suspension density set-point on separation performance for size fraction of $1.0-0.7 \mathrm{~mm}$

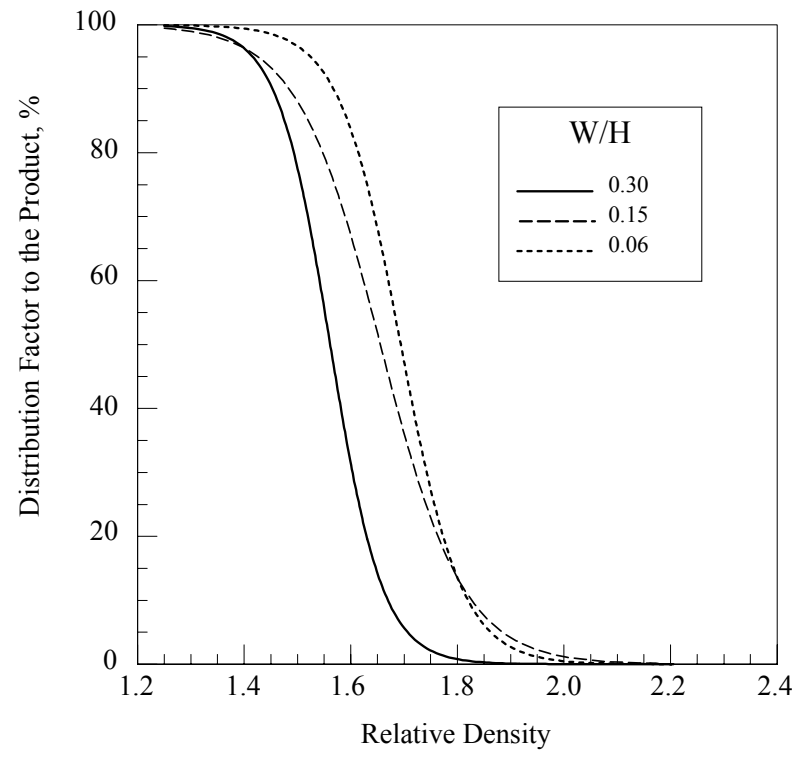

Figure 7.14 Effect of different width/height ratio on separation performance 
width/height ratio that might be caused by increased particle-wall collisions which produces a mixing effect and reduces the separation efficiency.

\subsubsection{Slurry Feed Velocity}

In the HSBS, the free settling velocities of particles are much smaller than its theoretical terminal free settling velocities because of particle-to-particle collisions at high solids volume and upward moving fluidization flow (Littler, 1986). Additionally, the high solids concentration increases the apparent viscosity and specific gravity of the slurry, thus increasing the buoyant force and resulting in a further reduction in particle settling velocities. The size and specific gravity of separation are expected to increase with slurry feed rate.

A series of simulations were conducted at various slurry feed rates. The settling of solid particles caused increased downward liquid flow velocity right below the feed inlet. The effect of solids flow on liquid velocity is exhibited in Figure 7.15. In case 6, the accompanying water of the feed slurry causes drastic changes in the flow pattern in the region near the feed inlet. The specific gravity of separation, SG50, increases with an increase in slurry velocity as shown in Figure 7.16, due to the additional upward flow from the feed slurry.

At constant slurry feed velocities of $0.026,0.065,0.104$ and $0.13 \mathrm{~m} / \mathrm{s}$, the corresponding specific gravity of separations, SG50s are 1.5615, 1.5089, 1.5098 and 1.4876, and the values of Ep at $0.0542,0.0822,0.1331$ and 0.1341 , respectively.

From these observations, adjusting the density cut-point by controlling fluidizing water velocity will be damped by extra water from the feed slurry. This is consistent with the postulation by Hanaker and Mondal (2000) that feed with dry materials lowers the density 


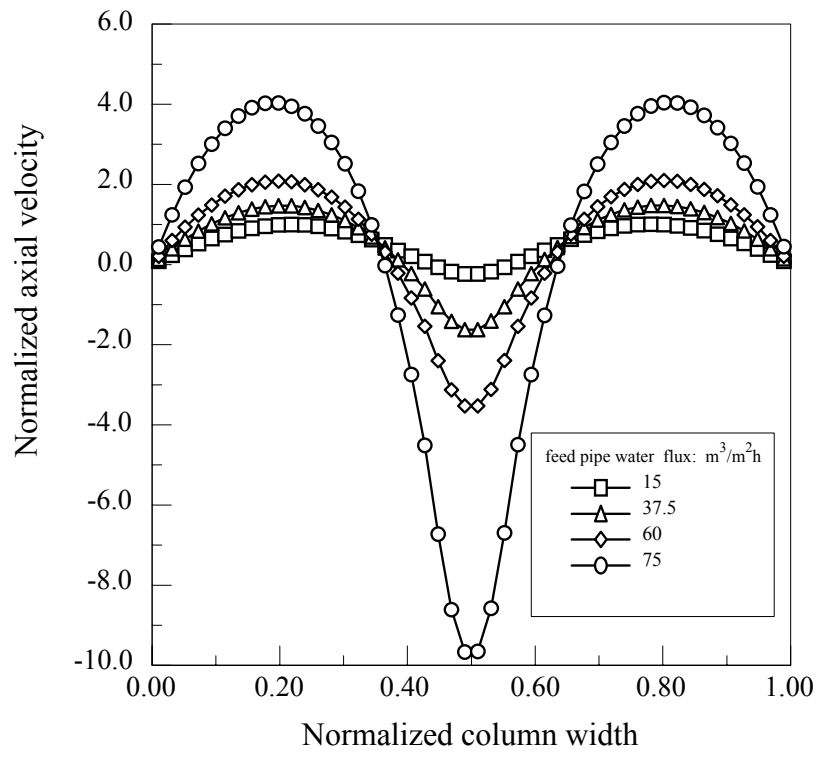

Figure 7.15 Effect of slurry feed rate on normalized axial velocity

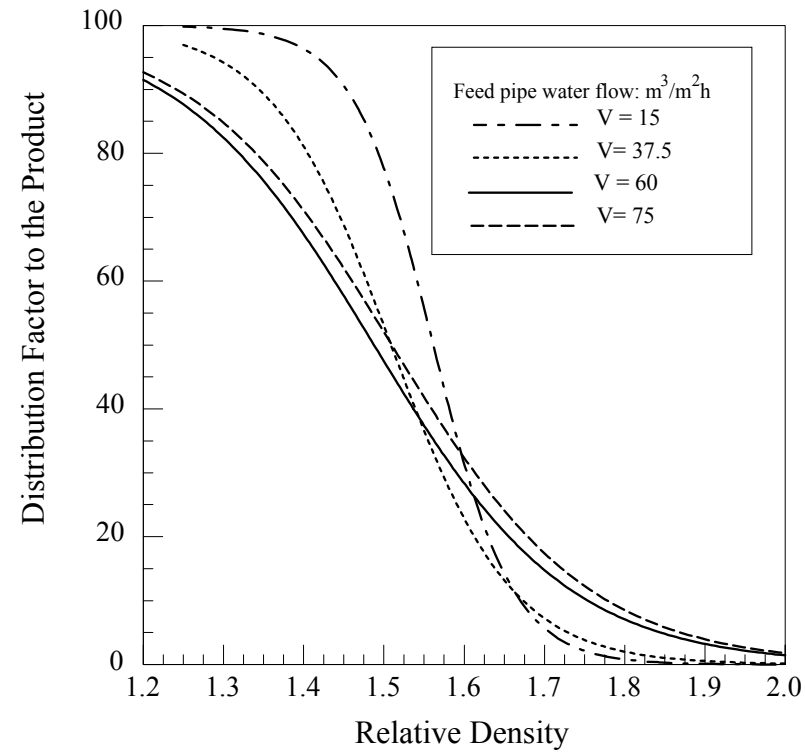

Figure 7.16 Effect of slurry feed rate on separation performance 
cut-point, and increases the capacity of hindered-settling bed separators.

\subsubsection{Solid Concentration of Feed}

Figure 7.17 shows that the axial liquid velocity changes with solid concentrations at a solid feed flow velocity of $21.4 \mathrm{t} / \mathrm{m}^{2} \mathrm{~h}$. The operating condition corresponds to case 7 as shown in Table 7.3. Figure 7.18 also shows that the density of separation increases with decreasing solid concentration. The density of separation is relatively unaffected by solid concentration until the solid concentration reaches $25 \%$. Above this level, the distribution factor curves flatten out, resulting in lower separation efficiency. At high solid concentration, the feed tends to plunge deeper into the HSBS, thus reducing the entrainment of heavy particles in the overflow and resulting in a lower SG50 value. By increasing the value of the density of separation, SG50, higher efficiency at low solid concentration may be due to the larger volume of water in feed slurry. This increases the upward flow velocity near the feed inlet region and carries more heavy particles in overflow while reducing the particle-particle collisions. The unit with a center tangential feeding system is more sensitive to the variation of solid feed rates if solid particles are fed as slurries rather than as dry feed. 


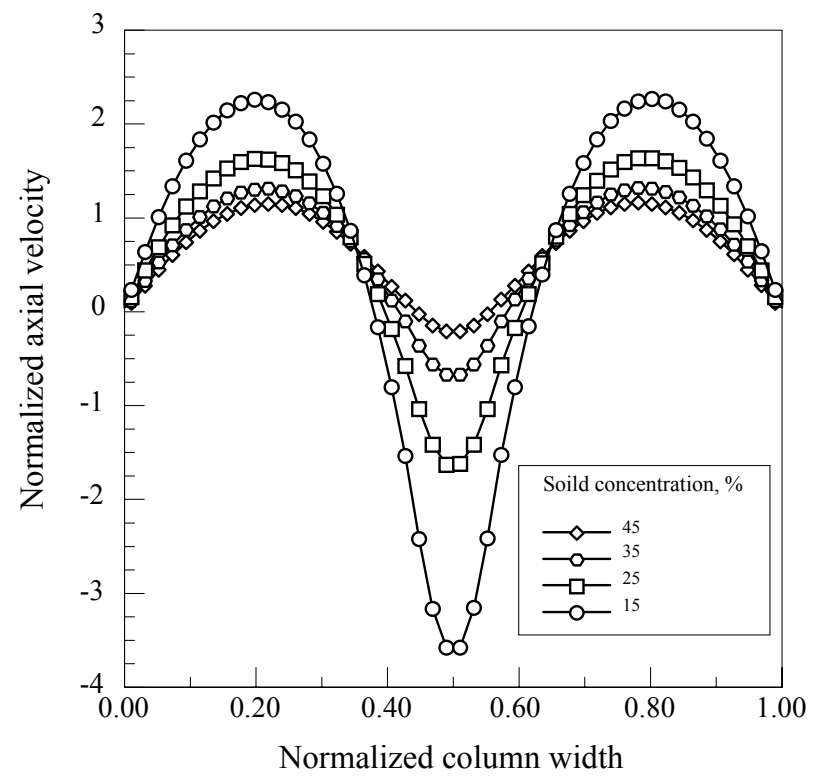

Figure 7.17 Effect of solid concentration of feed on normalized axial velocity

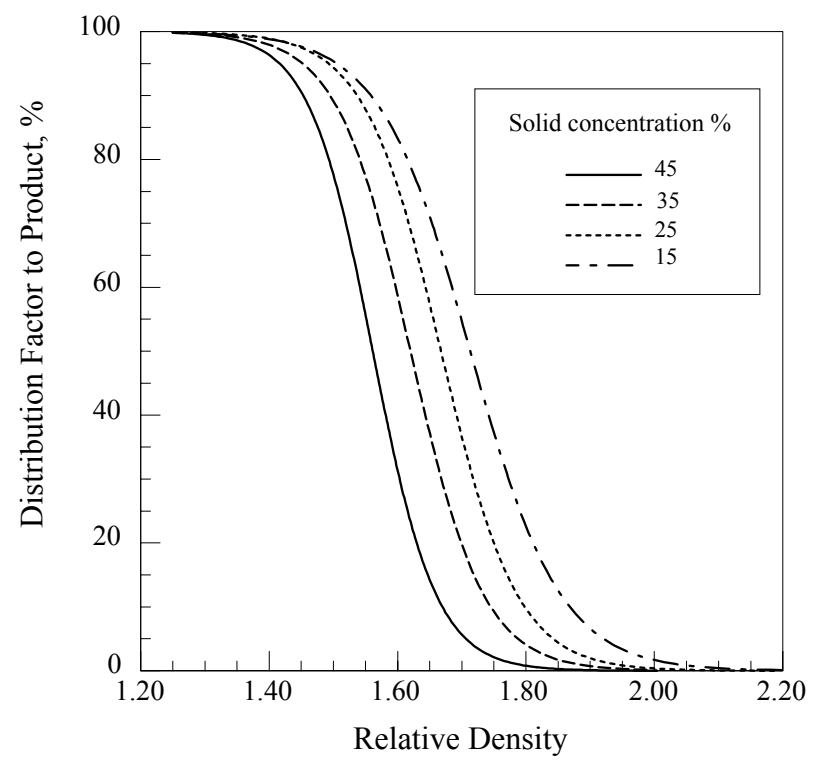

Figure 7.18 Effect of feed solid concentration on separation performance 


\subsection{Validation of Model}

In case 8 , the validity of the separator model developed by using the Euler-Lagrange approach in CFD is evaluated by comparison of the simulated results with those obtained from actual in-plant sampling test data for the hindered-settling bed separators. Tables 7.4 and 7.5 listed the input data and output data files. Figures 7.19 and 7.20 show that the predicted values and in-plant test data for the distribution factors of

Table 7.4 Input data in simulations

\begin{tabular}{|c|c|c|c|c|}
\hline \multirow{6}{*}{ Unit dimensions, $\mathrm{m}$} & \multicolumn{3}{|c|}{ Column diameter, $\mathrm{D}_{1}$} & 3.66 \\
\hline & \multicolumn{3}{|c|}{ Slurry feeding pipe diameter, $\mathrm{D}_{2}$} & 0.86 \\
\hline & \multicolumn{3}{|c|}{ Column height, $\mathrm{H}$} & 3.48 \\
\hline & \multicolumn{3}{|c|}{ Height of cylinder section, $\mathrm{H} 1$} & 3.0 \\
\hline & \multicolumn{3}{|c|}{ Depth of feeding pipe, $\mathrm{H} 2$} & 0.89 \\
\hline & \multicolumn{3}{|c|}{$\begin{array}{l}\text { Set point height from fluidizing water } \\
\text { feeding level }\end{array}$} & 0.70 \\
\hline \multirow[t]{11}{*}{ Size distributions, $\mathrm{mm}$} & \multicolumn{2}{|c|}{\begin{tabular}{l|l} 
Size, $\mathrm{mm}$ \\
\cline { 2 - 2 }
\end{tabular}} & \multicolumn{2}{|l|}{$\mathrm{Wt}, \%$} \\
\hline & \multicolumn{2}{|l|}{$-5.00+2.38$} & \multicolumn{2}{|l|}{5.68} \\
\hline & \multicolumn{2}{|l|}{$-2.38+1.68$} & \multicolumn{2}{|l|}{13.15} \\
\hline & \multicolumn{2}{|l|}{$-1.68+1.18$} & \multicolumn{2}{|l|}{15.07} \\
\hline & \multicolumn{2}{|l|}{$-1.18+1.00$} & \multicolumn{2}{|l|}{7.11} \\
\hline & \multicolumn{2}{|l|}{$-1.00+0.85$} & \multicolumn{2}{|l|}{8.03} \\
\hline & \multicolumn{2}{|l|}{$-0.85+0.60$} & \multicolumn{2}{|l|}{16.93} \\
\hline & \multicolumn{2}{|c|}{$-0.60+0.425$} & \multicolumn{2}{|l|}{13.72} \\
\hline & \multicolumn{2}{|c|}{$-0.425+0.30$} & \multicolumn{2}{|l|}{13.34} \\
\hline & \multirow{2}{*}{\multicolumn{2}{|c|}{$-0.30+0.212$}} & \multicolumn{2}{|l|}{6.97} \\
\hline & & & \multicolumn{2}{|l|}{100.0} \\
\hline \multirow[t]{10}{*}{ Density composition } & \multicolumn{2}{|c|}{ Density, $\mathrm{g} / \mathrm{cm} 3$} & $\mathrm{Wt}, \%$ & \\
\hline & $1.20-1.30$ & & 41.19 & \\
\hline & $1.30-1.40$ & & 21.19 & \\
\hline & $1.40-1.60$ & & 16.28 & \\
\hline & $1.60-1.80$ & & 3.92 & \\
\hline & $1.80-2.0$ & & 1.82 & \\
\hline & $2.00-2.20$ & & 2.87 & \\
\hline & $2.20-2.45$ & & 3.34 & \\
\hline & $2.45-2.80$ & & 9.39 & \\
\hline & & & 100.00 & \\
\hline $\begin{array}{l}\text { Fluidizing water velocit } \\
\text { (superficial fluidizing } \mathrm{w}\end{array}$ & $\begin{array}{l}\mathrm{m}^{3} / \mathrm{m}^{2} \mathrm{~h} \\
\text { er velocity, } \mathrm{mm} / \mathrm{s} \text { ) }\end{array}$ & $\begin{array}{l}24.12 \\
(6.7) \\
\end{array}$ & & \\
\hline Solid feed flow, $\mathrm{t} / \mathrm{m}^{2} \mathrm{~h}$ & & 12.27 & & \\
\hline Feed solid concentratior & & 45 & & \\
\hline $\begin{array}{l}\text { Water flow velocity in } \mathrm{f} \\
\text { (water velocity in feed } \mathrm{p}\end{array}$ & $\begin{array}{l}\text { ding pipe }, \mathrm{m}^{3} / \mathrm{m}^{2} \mathrm{~h} \\
\mathrm{e}, \mathrm{mm} / \mathrm{s})\end{array}$ & $\begin{array}{l}26.2 \\
(45)\end{array}$ & & \\
\hline Set point relative densit & $\mathrm{g} / \mathrm{cm}^{3}$ & 1.14 & & \\
\hline
\end{tabular}


size and specific gravity of separations, respectively. The results show that good agreement between the predicted results and plant test data are obtained. The specific gravity of separation, SG50 is 1.86 and Ep has the value of 0.061 for the in-plant test results for processing fine coal. The steeper shape of density distribution factor curves for the specific gravity of separation indicates that the separation is dominated by the specific gravity of the particles rather than by size of the particles.

Table 7.5 List of output data files

\begin{tabular}{|r|c||}
\hline Data file name & Description of date \\
\hline denPos.out & Particle position by density with time \\
\hline sizePos.out & Particle position by size with time \\
\hline Partition_D.out & Overall distribution factor to the product based on density \\
\hline Partition_S.out & Overall distribution factor to the product based on size \\
\hline Partition_SD.out & Distribution factor to the product based on density for each size fraction \\
\hline Fraction.out & Solid volume fraction \\
\hline Sus_den.out & Suspension density distribution \\
\hline SizeDistri.out & Feed size composition \\
\hline DenDistri.out & Feed density composition \\
\hline OpenU.out & Liquid axial velocity distribution \\
\hline OpenV.out & Liquid radial velocity distribution \\
\hline OpenP.out & Pressure distribution \\
\hline
\end{tabular}




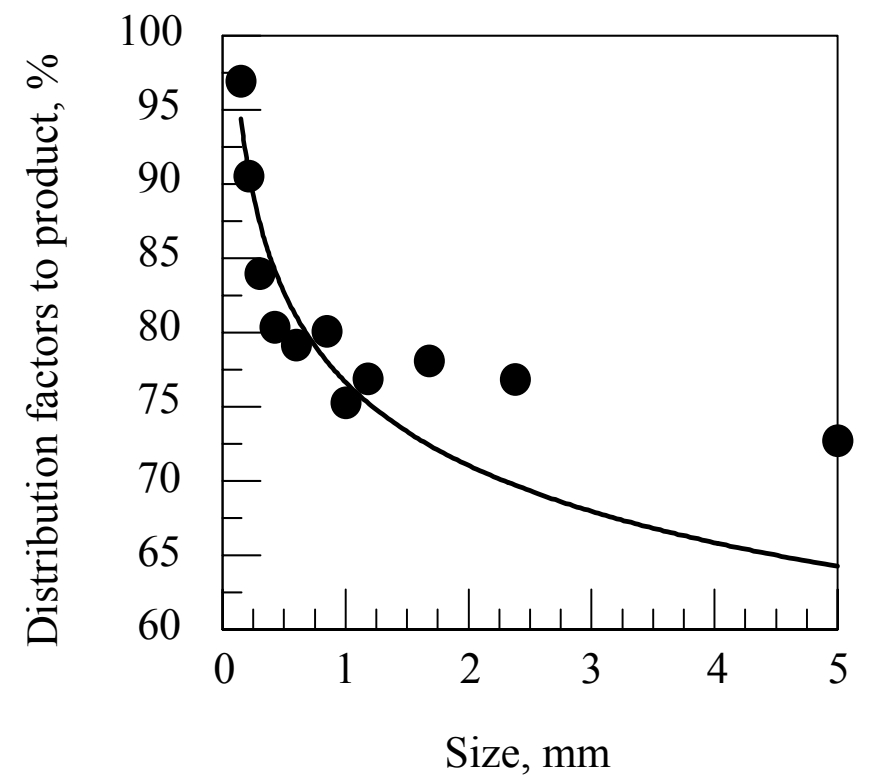

Figure 7.19 Distribution curves for size of separation for HSBS with a tangential feed system

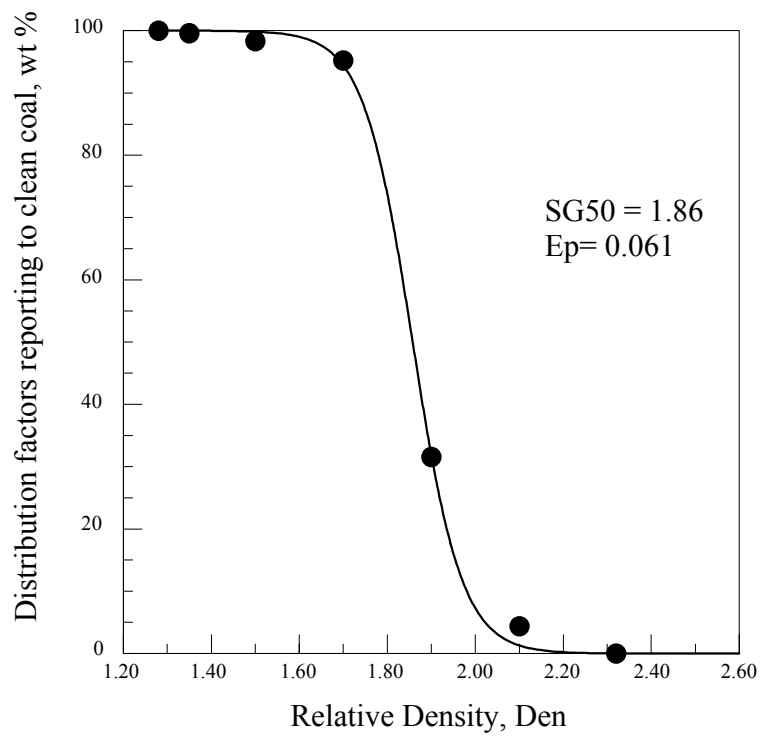

Figure 7.20 Distribution curve for specific gravity of separation for HSBS with a tangential feed system 


\subsection{Summary}

- A two-dimensional, two phase flow (solid-liquid) mathematical model using an Euler-Lagrange approach for a time-dependent calculation was used to investigate the particle separation mechanisms in hindered-settling bed separators.

- The fluid dynamic model is able to account for major geometric variables and operating parameters of the hindered-settling bed separator. Both liquid velocity profiles and solid particle movement can be predicted in detail.

- This mathematical model for the hindered-settling bed separators provides useful tools for evaluation of the performance of separation. The simulated results for size separation and specific gravity of separations are in good agreement with results obtained from in-plant test results for fine coal.

- According to the simulation, an increase in fluidizing water velocity, solid flow velocity, density set-point or decrease in feed solid concentration produces a higher density separation. The high volumetric flow velocity of water in the feed causes a significant impact on liquid flow pattern, which produces a product with higher density. Column width/height ratios has a minor effect on the density separation when ratio is higher than 0.15 . 


\section{Chapter 8 Influence of Structured Plates in a Liquid Fluidized Bed System}

The addition of structured plates in the HSBS is a new design for density separation on the basis of size and density. This work presents a series of numerical simulations of the particle classification and density separation in the presence of structured plates using an Euler-Lagrange approach from Computational Fluid Dynamics (CFD). In the simulation, the HSBS was used to separate coal particles within a size range of $-2+0.25 \mathrm{~mm}$. The changes in the liquid phase velocity field and the separation performance in the presence of structured plates are compared with the open column values in order to obtain a better understanding of the contributions from the structured plates in coal separation.

\subsection{Fine Coal Density Separator}

In fine coal preparation, the clean coal particles have low density and contain a low ash content. The refuse is high density mineral matter which has a high ash content. A separation of clean coal from the refuse can be made using density separation within certain size ranges. The current technology used in coal preparation plants for recovering fine coal in the $2.0+0.25 \mathrm{~mm}$ size range includes spirals and dense medium cyclones. Although spirals are relatively less expensive to operate, they are limited in their operating density cut-point, the specific gravity of separation, SG50, greater than 1.7. They also suffer from feed distribution problems, capacity limitations and relatively high probable errors (Ep). Dense medium cyclones offer improved performance, but usually at a higher cost because of the need to use and recover the magnetite medium. 
Ideally, the density separation in the HSBS should be based on the density difference for a feed with a narrow size range, with only low density clean coal particles reporting to the overflow and high density particles to the underflow. In practice, coal particles tend to have a broad size range in the feed. Although a HSBS has a good separation quality if particle size is within a well-defined sized range. If the particles are too fine, the separation quality deteriorates. The fine refuse with a high density easily reports to the overflow by elutriation while coarse clean coal with a low density can be lost in the underflow. In order to minimize this negative effect, the fluidizing water velocity must be keep at the lowest possible level to maintain a suspension concentration. However, the low fluidizing water velocity limits the throughput of the separator and also results in the loss of coarse, low density coal in the underflow.

In recent years, structured plates have been incorporated in a large variety of industrial equipment to improve fluid-dynamic behavior, enhancing efficiency and lowering energy costs. In mineral processing, for instance, the use of structured plates proved to increase the solid capacity, reduce water throughput, enhance density separation performance and save energy consumption. In mineral processing, the use of structured plates in mineral processing equipment based on the principles of hindered-settling velocity proved to increase the solid capacity, to reduce water throughput and to enhance density separation performance in relation to a conventional HSBS.

The addition of parallel inclined plates or structured plates inside the HSBS is a solution to the previously mentioned throughput limitation problem. The installation of such a series of inclined plates in the HSBS can greatly improve the separation performance and increase the throughput. Galvin et al. (2002) have investigated the interaction of a set of parallel 
inclined plates and a fluidized suspension in the Reflux classifier. One advantage of this equipment is that Reflux can maintain a stable suspension concentration with the aid of inclined plates for a broad range of fluidizing water velocities. It was observed that, at high solid feed rate, the extra particles can reach the upward faces of the inclined plates and slide downward to fluidized-bed zone below the inclined plates. Thus, the extra particles in the high feed concentration can be extracted and maintain a stable suspension density.

The addition of radial or helical plates such as static mixers can also improve coal and mineral separation. Dai (1999), Peng et al. (2002) and Yang (2002) conducted a series of tests treating coal and mineral samples in a column jig. The differences of a column jig from the HSBS lies in that there is a pulsating water flow in a HSBS with structured plates while only a steady state fluidizing water flow exists in an open hindered-settling column. From their experimental work, it was found that column jig shows a good separation for fine iron particles at high throughput and efficiency.

Since the inclusion of baffles or structured plates have improved the fine particle separation with increased throughput and lowered separation size limit from the above experiments, it is of interest to determine the hydrodynamic behavior of structured plates in separation and to compare the corresponding values with those of the open column. An Euler-Lagrange multiple phase model is applied to a HSBS with structured plates. The liquid flow patterns and particle density separation are directly simulated.

\subsection{Laboratory Performance Tests}

\subsubsection{Experiment}

A laboratory-scale HSBS was used to investigate the separation of a feed consisting of fine particles. A bench scale 2-in diameter pipe and 30-in high hindered-settling bed 
separator with structured plates was used to study the effect of structured plates. The schematic diagram of the system is shown in Figure 8.1.

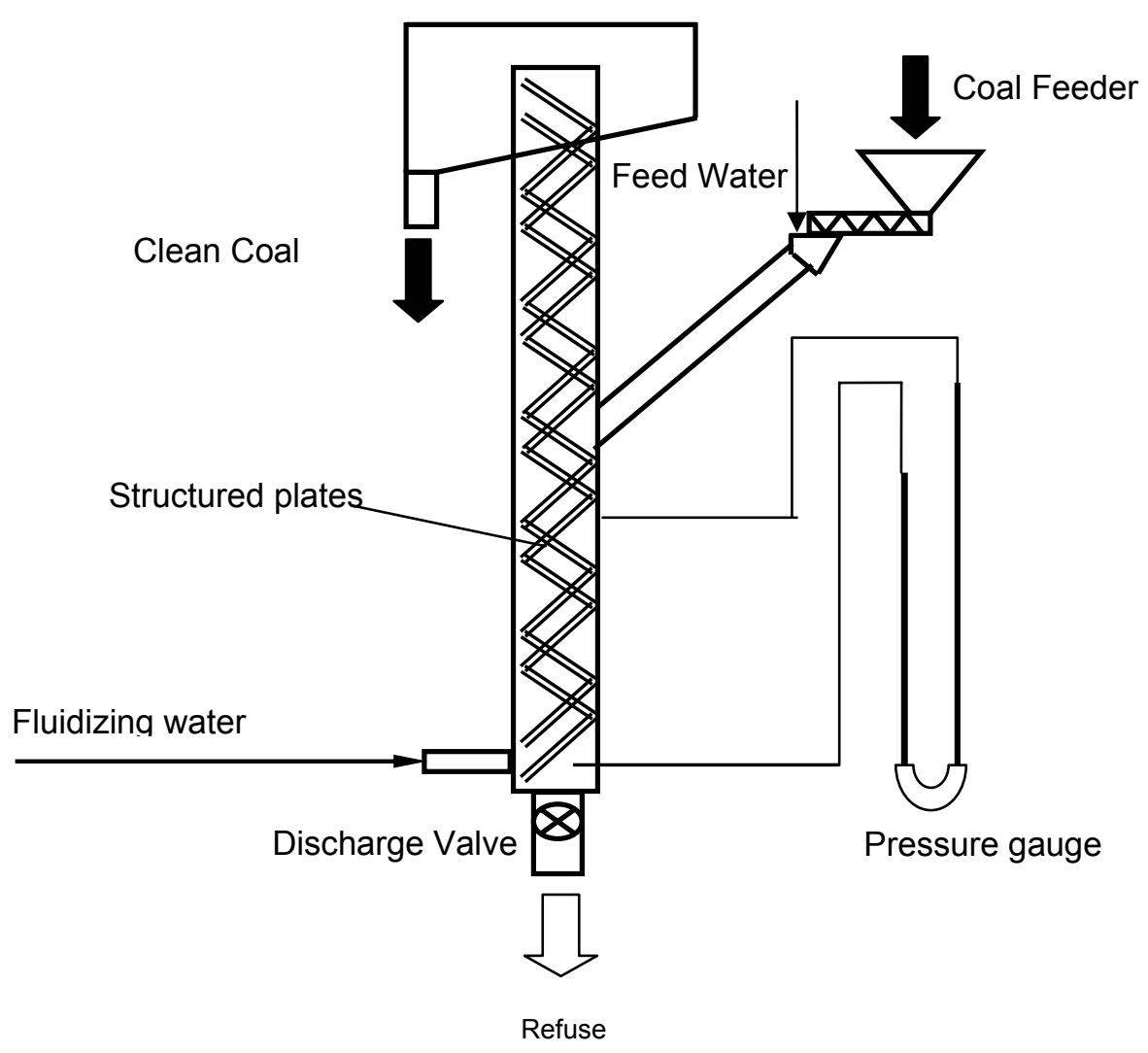

Figure 8.1 Schematic diagram of a HSBS with structured plates

The HSBS is connected to a $\mathrm{U}$ tube pressure gage for monitoring the pressure gradient. The HSBS is made of transparent PVC pipe, thus allowing the bed level to be observed. The fluidizing water velocity is controlled by flow meters. A perforated plate was used at the base to produce a uniform fluidization flow condition. The underflow is manually controlled and heavy particles are discharged to a 5 gallon pail. The overflow from the fluidized-bed reported to a 33 gallon drum, allowing the recovery of the light particles. The structured 
plates are made of three $0.3 \mathrm{~mm}$ stainless steel corrugated plates. The plate had a fold angle, $\alpha=120^{\circ}$, height corrugation, $\delta p=30 \mathrm{~mm}$, corrugated angle, $\beta=45^{\circ}$, bended diagonally, and the distance between neighboring plates, $\mathrm{D}=18 \mathrm{~mm}$.

\subsubsection{Experiment Results}

The laboratory testing results of the open column and the baffled column are shown in Table 8.1 . The fluidizing water velocity is kept at $1.5 \mathrm{~cm} / \mathrm{second}$ and the density set point is kept at 1.10. The feed properties are shown in Tables 7.1 and 7.2. At solid feed rate of 10 $\mathrm{t} / \mathrm{m}^{2} \mathrm{~h}$, the combustible material recovery of $94.11 \%$ and $95.06 \%$ are obtained for the open column and baffled column repectively. They have equal combustible material recovery. Although the clean coal product in the open column has a lower clean coal ash content, the refuse ash content is only $63.31 \%$. That means a substantial amount of clean coal particles are lost in refuse in case of open column. The clean coal product in the baffled column has a little higher ash content than that in the open column; However, the refuse ash content is as high as $75.80 \%$. Thus, under same operating conditions, the baffled column can minimize the clean coal particles loss in refuse. If the solid feed rate is increased from 10 to $30 \mathrm{t} / \mathrm{m}^{2} \mathrm{~h}$, the density separation in the open column deteriorates and shows a very low refuse ash content (48.24\%). In case of the baffled column, it maintains a relatively good separation at this high level of solid feed rate. The baffled column produces a lower clean coal ash content $(12.69 \%)$ and a higher refuse ash content (61.66\%). The combustible material recovery of the clean coal product from the baffled column is also higher than that in the open column. Thus, the baffled column can operate at a much higher solid feed rate level without lost much separation performance. 
Table 8.1 Density separation performance comparison between open column and baffled column

\begin{tabular}{||l|l|l|l|l|l|l||}
\hline $\begin{array}{l}\text { Feed rate, } \\
\mathrm{t} / \mathrm{m}^{2} \mathrm{~h}\end{array}$ & $\begin{array}{l}\text { Column } \\
\text { type }\end{array}$ & $\begin{array}{l}\text { Raw coal } \\
\text { ash \% }\end{array}$ & $\begin{array}{l}\text { Clean coal } \\
\text { yield \% }\end{array}$ & $\begin{array}{l}\text { Clean coal } \\
\text { ash \% }\end{array}$ & $\begin{array}{l}\text { Refuse ash, } \\
\%\end{array}$ & $\begin{array}{l}\text { Combustible } \\
\text { material } \\
\text { recovery \% }\end{array}$ \\
\hline 10 & open & 21.37 & 86.96 & 12.07 & 63.31 & 94.11 \\
\hline 10 & baffled & 21.37 & 84.32 & 13.46 & 75.80 & 95.06 \\
\hline 30 & open & 21.37 & 80.79 & 14.80 & 48.24 & 87.38 \\
\hline 30 & baffled & 21.37 & 86.22 & 12.69 & 61.66 & 93.40 \\
\hline
\end{tabular}

* Clean coal product and refuse are de-slimed

\subsection{Results and Discussions for Simulation}

\subsubsection{Solid-Liquid Flows}

\subsubsection{Flow Patterns}

In order to observe the flow patterns difference in the open column and baffled column, and also be able validate the simulation results, the columns with and without structured plates are built as shown in Figures 8.2(a) and 8.2(b) respectively.

The liquid flow patterns in both the open column and the baffled column with the addition of the structured plates are simulated when no particles are added as shown in Figure 8.2. Figures 8.3 to 8.8 show the steady liquid flow patterns in different columns, which are presented both in magnitude contour and velocity vector maps. At a superficial fluidizing water velocity of $7 \mathrm{~mm} / \mathrm{s}$, a down stream vortex develops at each corner of the open column at the liquid inlet as shown in Figures 8.3 to 8.5. There also exists a symmetric flow pattern in open column with a maximum velocity along the central line. 


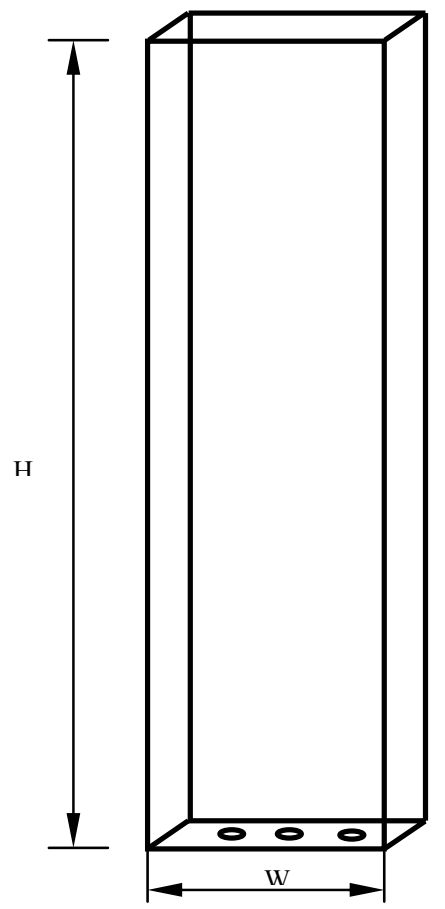

(a) Open column

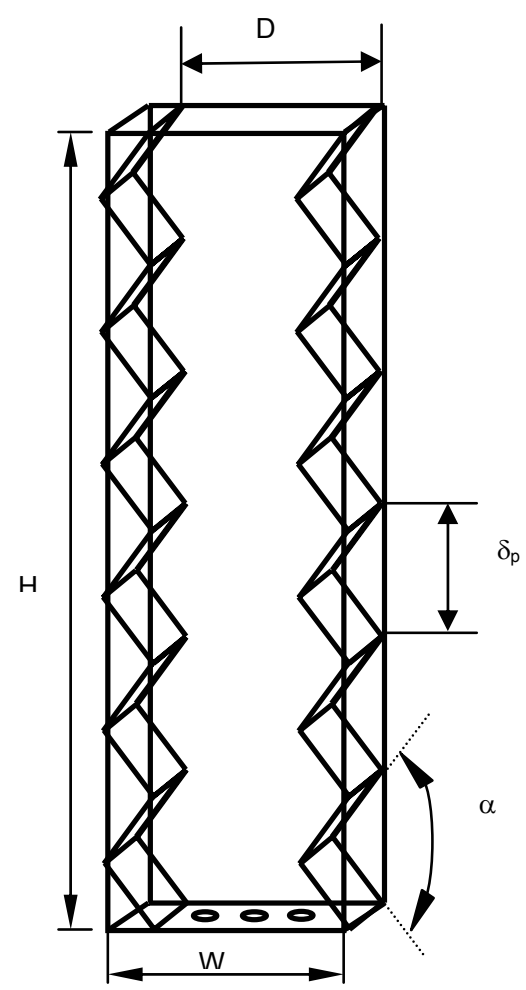

(b) Baffled Column

Figure 8.2 Schematic diagrams of open and baffled columns in simulation

The liquid flow inside column with structured plates is somewhat different in that the downstream vortices developed at inlet end and behind each indent corners as shown in Figures 8.6 to 8.8. The fluid is forced to flow along the zigzag shape of the plates, but only small vortices begin to form at each sharp bending corner. The liquid is rotating in each indent corner. The maximum velocity exists on the zigzag center line which follows the shape of the structured plates. Because of the characteristics of the structured plates, the plates reduce the flow vortex size in the column and the whole column can be considered as a network of small virtual cells. Thus the separation performance is an integration of the separation from each virtual cell. 

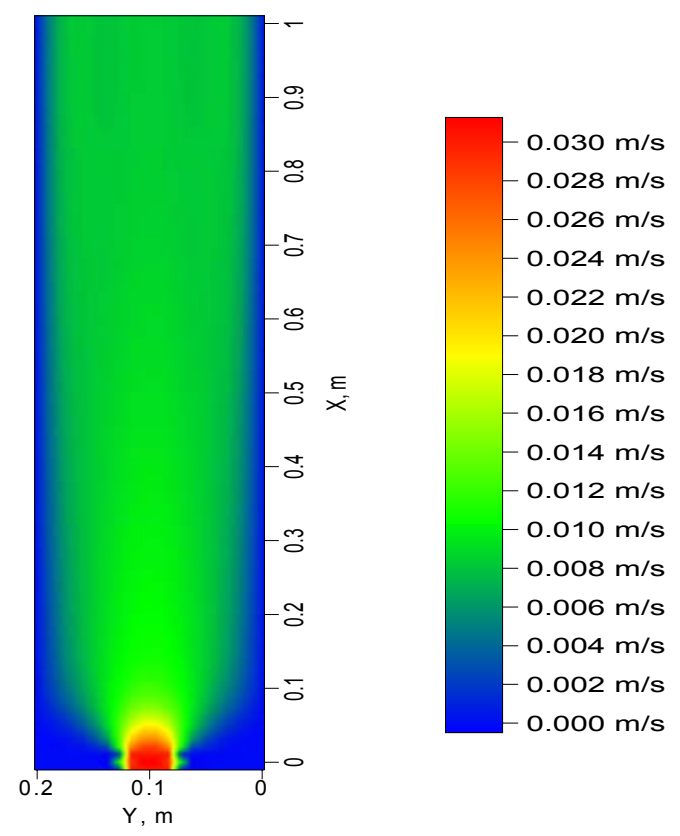

Figure 8.3 Axial velocity magnitude map in an open column
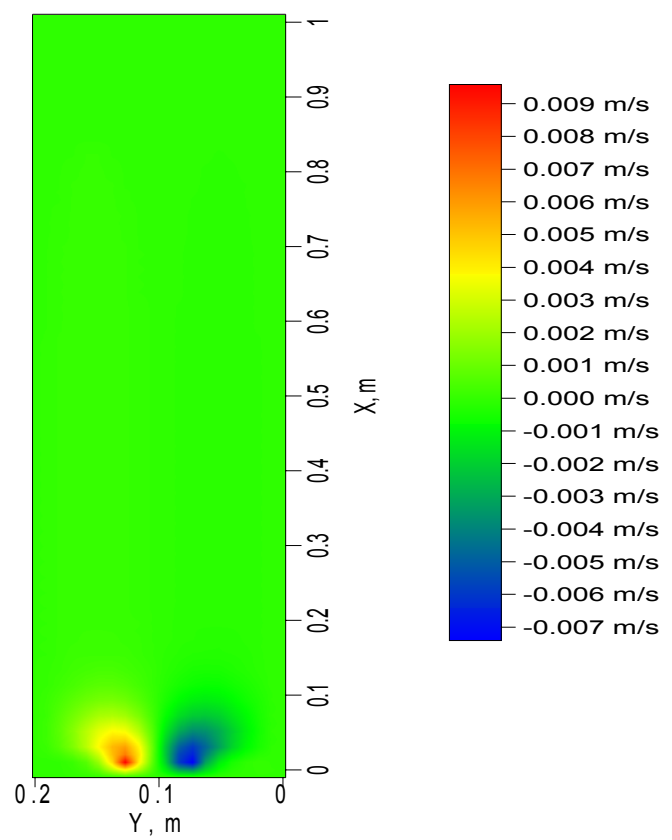

Figure 8.4 Horizontal velocity magnitude map in an open column 


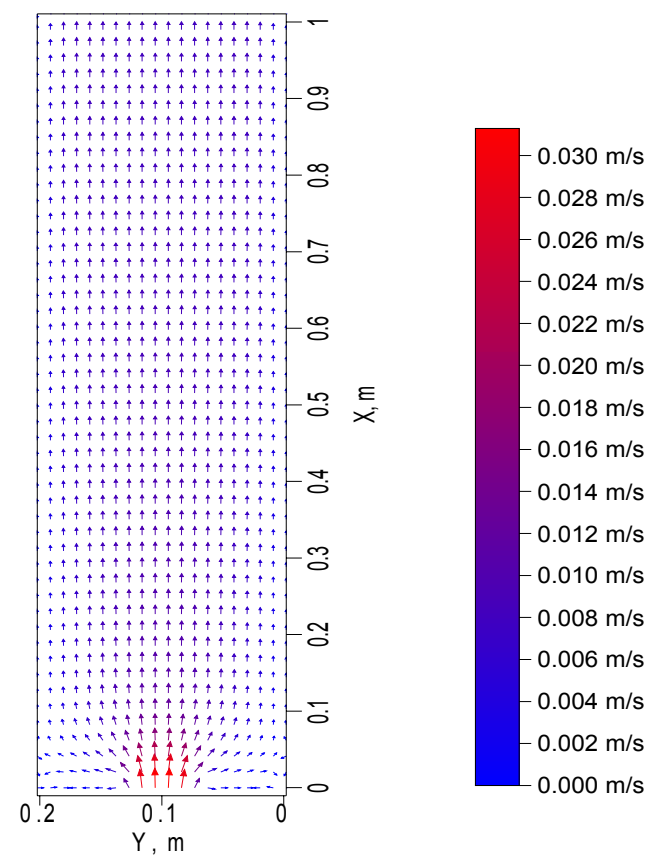

Figure 8.5 Velocity vector map in an open column
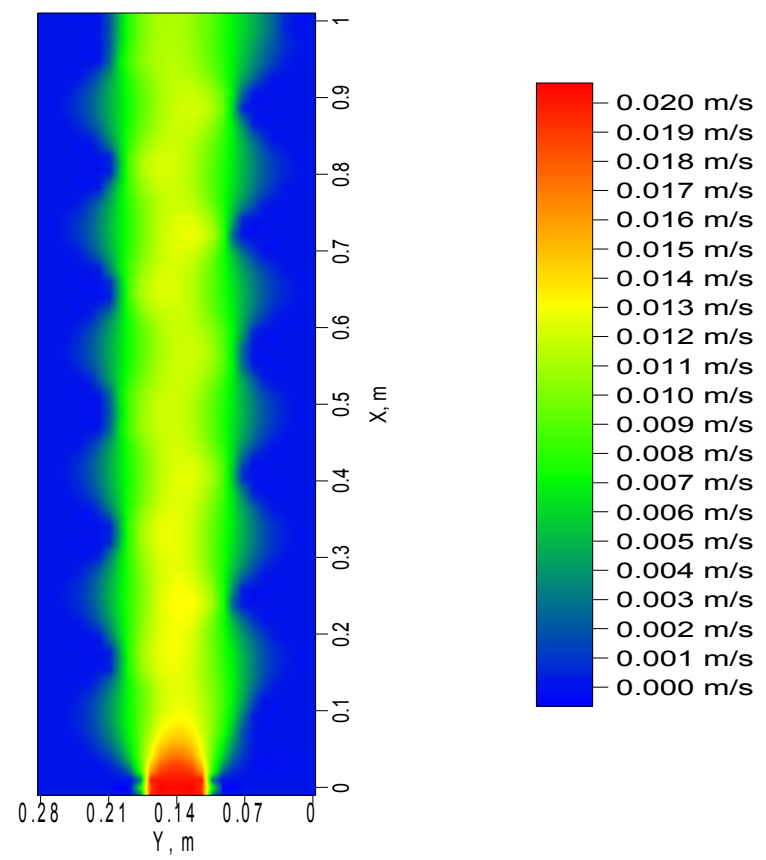

Figure 8.6 Axial velocity magnitude map in a baffled column 

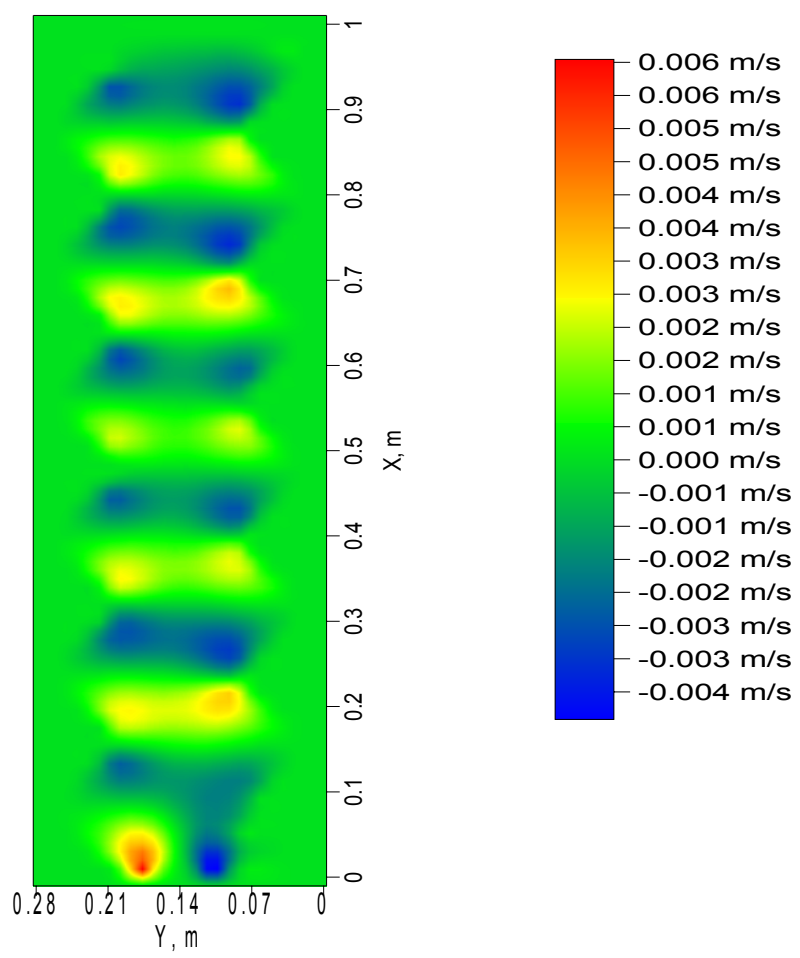

Figure 8.7 Horizontal velocity magnitude map in a baffled column
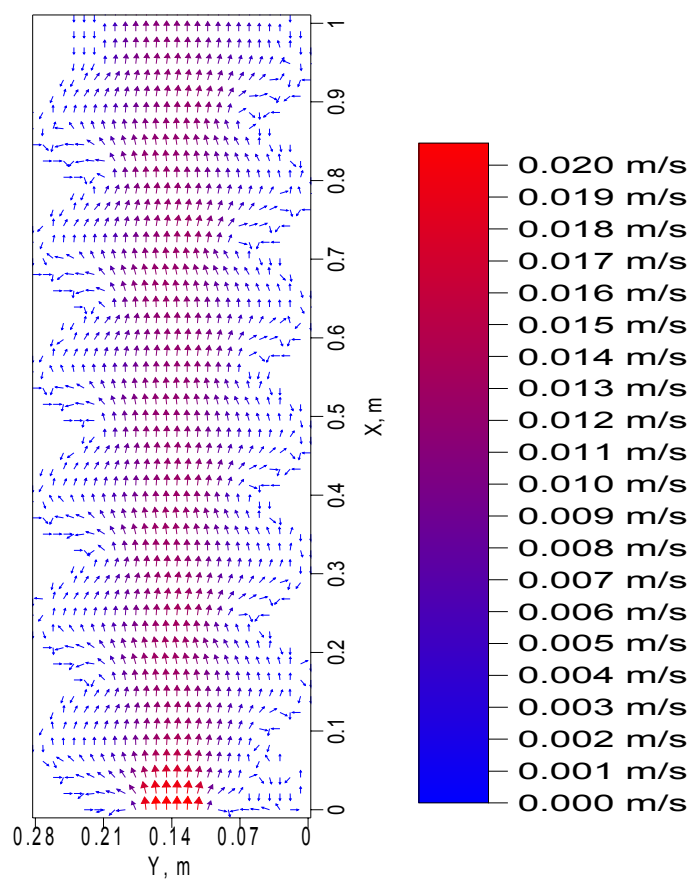

Figure 8.8 Velocity vector map in a baffled column 


\subsubsection{Particle Separation}

The addition of inclined plates has been used traditionally in solid-solid and solid-liquid separations. At very low liquid flow velocity, as the flow passes through the inclined plates, the solid particles will settle onto the plates and slide down along plate surface, while clear water overflows at the top of the inclined plates. The addition of inclined plates speeds up the settling of particles because of the shorter distance between

plates, and further increase the throughput of separator. The same concept of design is introduced in the design of advanced HSBS to improve capacity and separation performance (Galvin et al., 2002).

In order to demonstrate the effect of the addition of structured plates, the flow patterns and particle separation performance are computed and directly simulated in both columns. In the baffled column, the liquid phase flow and discrete particle movement inside the channel between two neighbored structured plates are directly computed.

A simulation of coal particle separation is made in both open column and baffled column containing a set of structured plates. The superficial velocity in both columns takes a value of $7 \mathrm{~mm} / \mathrm{s}$ which is equivalent to $25.2 \mathrm{~m}^{3} / \mathrm{m}^{2} \mathrm{~h}$. The particles have a uniform size of $1 \mathrm{~mm}$ and an evenly distributed density compositions between 1.2 and $2.8 \mathrm{~g} / \mathrm{cm}^{3}$. The solid feeding velocity is $2.65 \mathrm{t} / \mathrm{m}^{2} \mathrm{~h}$. Figure 8.9 compares the suspension density peak values at different heights along the columns and Figure 8.10 shows instantaneous particle positions in the baffled column at simulation time $t=300$ seconds. The coarse particles settle on the upward faces of inclined plates. These heavy particles can slide down along the surface because of gravity and the small friction coefficient in the liquid phase. The slower settling particles which consist of fine and lighter particles will emerge through the top of the plates and are 
carried out by fast moving flow in the middle of the channel between the two structured plates. Particles are thus separated by density differences.

From experimental observations and visual display of particle movement in the animation files from the simulation results, two characteristics of particle separation in the presence of structured plates are revealed here:

\section{a. Structured plates help to build a suspension density inside the structured}

\section{plates}

It is shown in Figure 8.9 that baffled columns can build a larger density gradient along the axial direction than in the open column. It is also noted that at the bottom of the baffled column the suspension density is much higher than that in the open column. This is consistent with the fact that coarse, low density particles are easily lost to tailings in the open column.

In the open column, all the particles are suspended only by the upward fluidizing water. However, when structured plates are added, the particles are also supported by upward faces of structured plates. The particles could have collisions with structured plate surfaces during upward or downward movements. In each unit of structured plates, heavy particles segregated from the slurry and settle onto the plates. The sediment formed by coarse high density particles then slides down the plates, returning to the fluidized-bed below. The light particles are carried upward to above the zigzag unit by the relative faster flow in middle of channel. The particles which have a middle density will accumulate inside the bended areas or corners of plate and build a relative high suspension density along the column. 


\section{b. Improved density separation by structured plates}

When the fast settling particles slide down the faces of plates, these particles can also trap some fine and light particles. The fast sediments are dispersed by upward flow at the sharp tips (bended corners) of plates. Only the heavy particle which has a higher settling velocity than the upward flow velocity can continue to settle down to the bottom of the column. The trapped light or fine particle will be carried upward again by fluidization water flow, mix with the rest of the suspension and, in time, are fed back up into the channels to be recovered. In principle, this internal reflux of particles should permit a refinement in the quality of the fine particle density separation.

Within each corner of the structured plate, the liquid flow is rotating. From animation of simulation results shown in Figure 8.10, the clean coal particles move up while rotating. The heavy particles settle down along the slope, at the tip of the structure plate. Particles are then carried upward by fast flow in the middle of channel and settle back toward inclined plate surfaces again. The particle flux is rotating within the bend area. At high solid concentrations, different particles are unavoidably mixed and trapped in wrong particle groups such as a heavy particle inside a block of light particle or vice versa. This particle rotation can provide some refinement of the fine particle density separation. In rotations, the trapped heavy particle can be released at the tip of bending of the structured plates; on the other hand, the trapped clean particle can also be elutriated at the tip of the bend structured plates. 


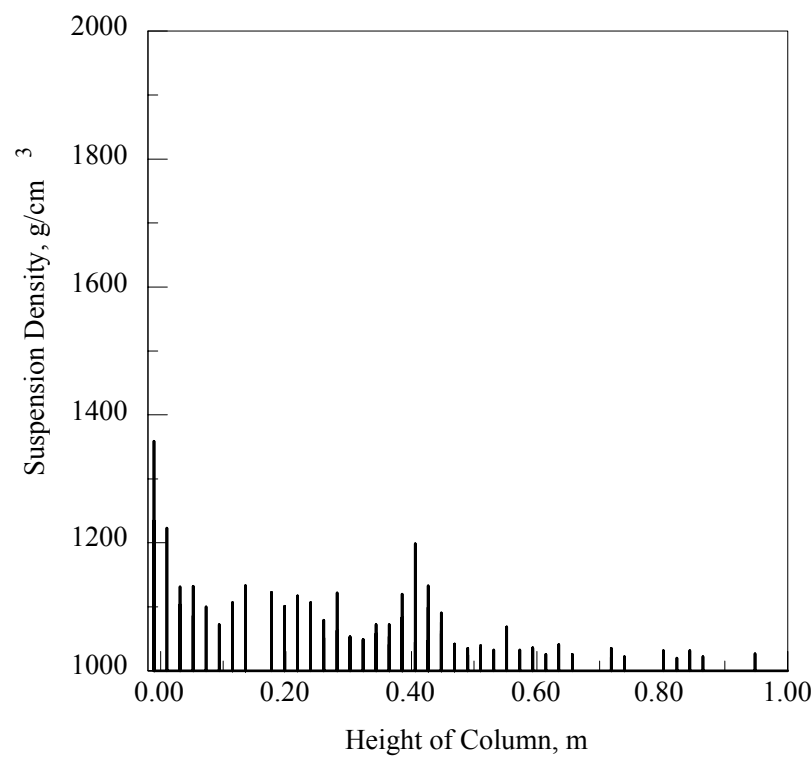

(a) Open column

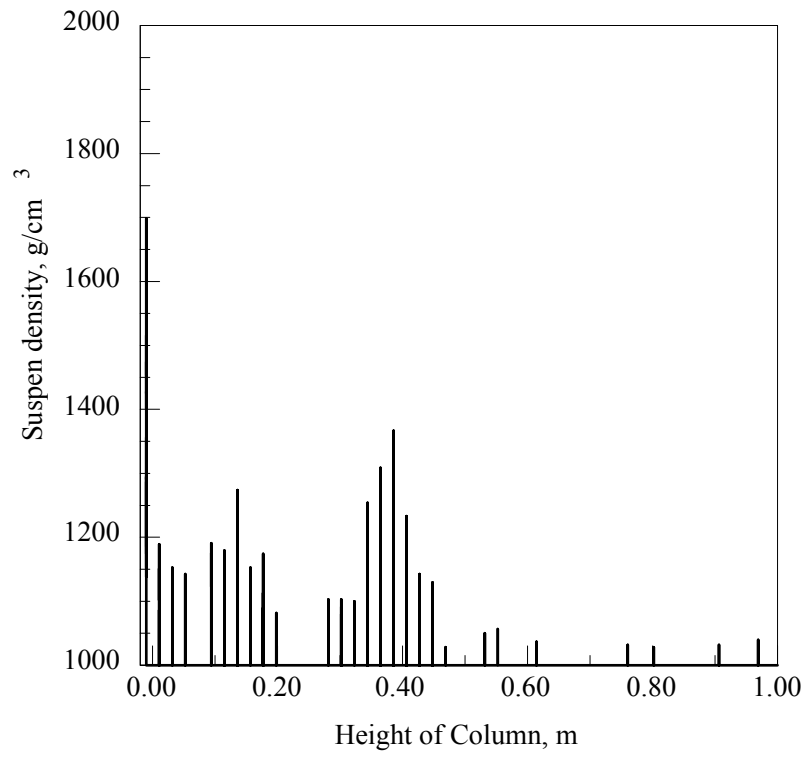

(b) Baffled column

Figure 8.9 Axial suspension density peak values at different heights along column 
In general, the structured plates have a profound impact on the behavior of the movement of fluid flow and the particles. The structured plates support the particle weight, channel and distribute the fluidizing water, form a high suspension density along the column and refine the separation through the rotations of liquid and the particles.

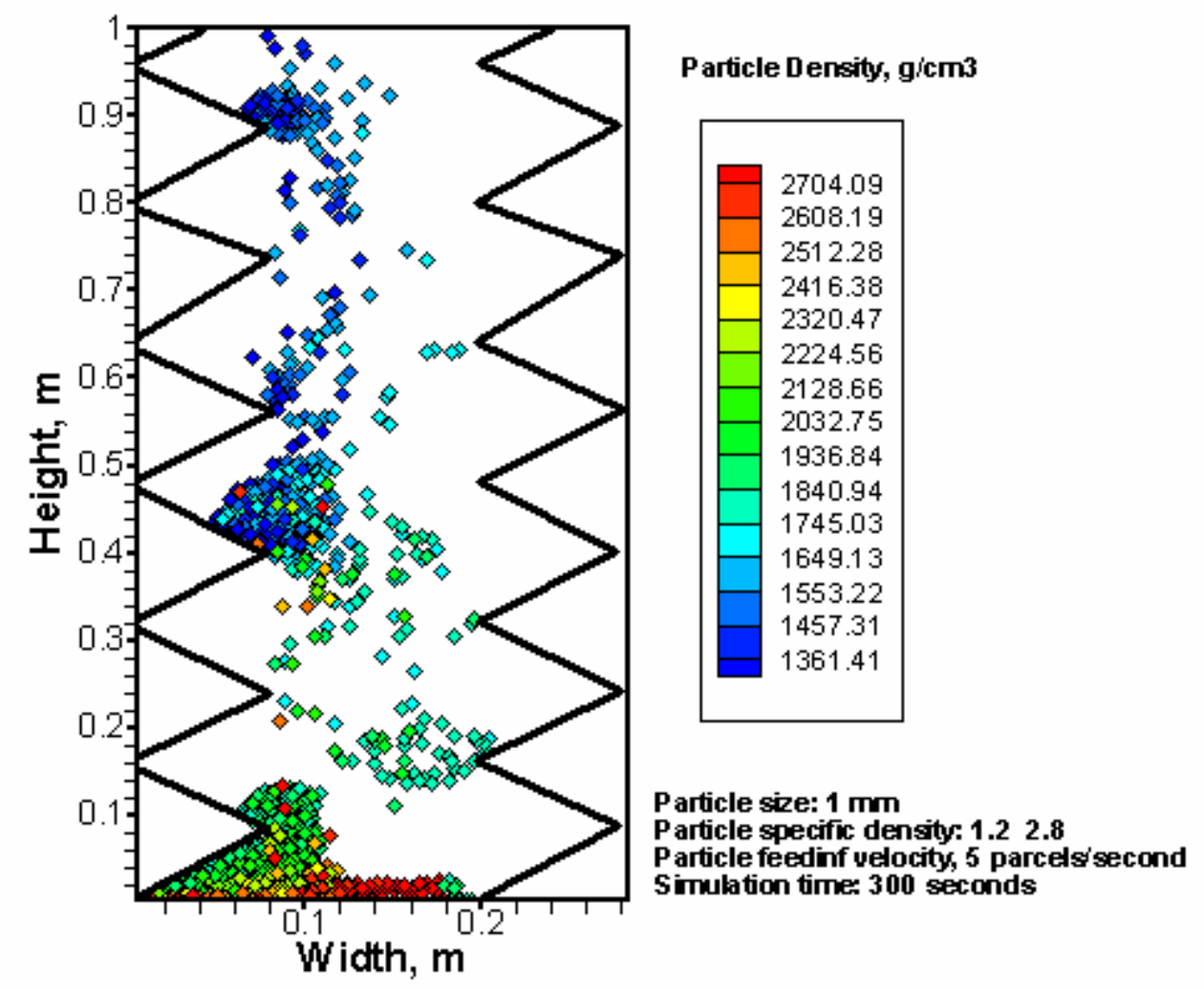

Figure 8.10 Particle stratification in a baffled column 


\subsubsection{Operating parameters}

\subsubsection{Fluidizing Water Velocity}

Nguyentranlam and Galvin (2001a, b) found that the fluidizing flow velocity can be many times greater than the terminal velocity of isolated particle in the presence of the parallel inclined plates while maintaining the same separation performance as the open column. Thus, the separator can run with a high throughput relative to a typical hinderedsettling bed separator. It was also concluded that the particles tend to form a relative high suspension density in spite of a possible high fluidizing water velocity.

Figures 8.11 and 8.12 show the effects of increasing the fluidizing water velocity on the density separation in different columns for particles with a uniform size of $1 \mathrm{~mm}$ at a set point suspension relative density of 1.05 . The simulation parameters used are listed in Table 8.1 and simulation results of separation index are described in Table 8.2. Clearly, at this suspension density, as shown in Figure 8.11, the $1 \mathrm{~mm}$ particle has a low specific gravity of separation, SG50 values, but with high Ep values at low fluidizing water velocity.

Table 8.2 Parameters used in simulation of the fluidizing water velocity effect at low suspension density

\begin{tabular}{|l|l|}
\hline Particle size & $1 \mathrm{~mm}$ \\
\hline Particle density & $1.2-2.8 \mathrm{~g} / \mathrm{cm}^{3}, \mathrm{wt} \%$ evenly distributed \\
\hline Inlet water flow velocity & $12.96-45.5 \mathrm{~m}^{3} / \mathrm{m}^{2} \mathrm{~h}$ \\
\hline Superficial fluidizing water velocity & $3.6-12.6 \mathrm{~mm} / \mathrm{s}$ \\
\hline Dry particle feed loading & $2.65-9.28 \mathrm{t} / \mathrm{m}^{2} \mathrm{~s}$ \\
\hline $\begin{array}{l}\text { Coefficient of restitution-particle } \\
\text { collisions }\end{array}$ & 0.1 \\
\hline Coefficient of restitution-wall collisions & 0.1 \\
\hline Friction coefficient & 0.1 \\
\hline Pulsation addition & No \\
\hline Suspension relative density at set point & $1.05 \mathrm{~g} / \mathrm{cm}^{3}$ \\
\hline Set point height & $0.35 \mathrm{~m}$ \\
\hline Simulation time & $30 \mathrm{~seconds}$ \\
\hline
\end{tabular}


Table 8.3 SG50 and Ep for different columns at different inlet water flow velocity

\begin{tabular}{||c|c|c|c|c|c|c|c|c||}
\hline $\begin{array}{c}\text { Inlet feed water } \\
\text { velocity }\end{array}$ & \multicolumn{2}{|c|}{$\mathrm{U}=3.6 \mathrm{~mm} / \mathrm{s}$} & \multicolumn{2}{c|}{$\mathrm{U}=5.4 \mathrm{~mm} / \mathrm{s}$} & \multicolumn{2}{c|}{$\mathrm{U}=9.0 \mathrm{~mm} / \mathrm{s}$} & \multicolumn{2}{c||}{$\mathrm{U}=12.6 \mathrm{~mm} / \mathrm{s}$} \\
\hline Open column & $\mathrm{SG} 50$ & $\mathrm{Ep}$ & $\mathrm{SG} 0$ & $\mathrm{Ep}$ & $\mathrm{SG} 0$ & $\mathrm{Ep}$ & $\mathrm{SG} 50$ & $\mathrm{Ep}$ \\
\cline { 2 - 10 } & 1.2359 & 0.4690 & 1.5254 & 0.3848 & 1.8952 & 0.2371 & 2.0509 & 0.1701 \\
\hline $\begin{array}{c}\text { Inlet feed water } \\
\text { velocity }\end{array}$ & $\mathrm{U}=5.04 \mathrm{~mm} / \mathrm{s}$ & $\mathrm{U}=7.56 \mathrm{~mm} / \mathrm{s}$ & $\mathrm{U}=12.6 \mathrm{~mm} / \mathrm{s}$ & $\mathrm{U}=17.64 \mathrm{~mm} / \mathrm{s}$ \\
\hline $\begin{array}{c}\text { Baffled column } \\
\text { without pulsation } \\
\text { flow }\end{array}$ & $\mathrm{SG} 50$ & $\mathrm{Ep}$ & $\mathrm{SG} 50$ & $\mathrm{Ep}$ & $\mathrm{SG} 50$ & $\mathrm{Ep}$ & $\mathrm{SG} 50$ & $\mathrm{Ep}$ \\
\cline { 2 - 10 } & 1.3786 & 0.3162 & 1.6200 & 0.1077 & 1.9248 & 0.0551 & 2.2911 & 0.095 \\
\hline
\end{tabular}

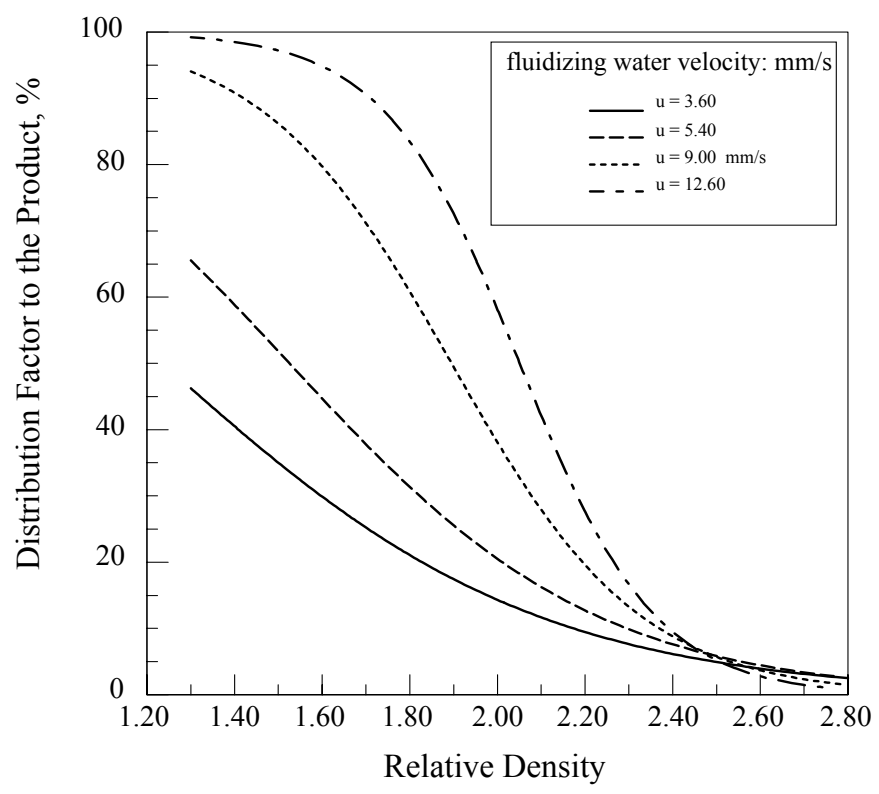

Figure 8.11 Distribution curves at various fluidizing water velocities in an open column (particle size: $1 \mathrm{~mm}$ ) 


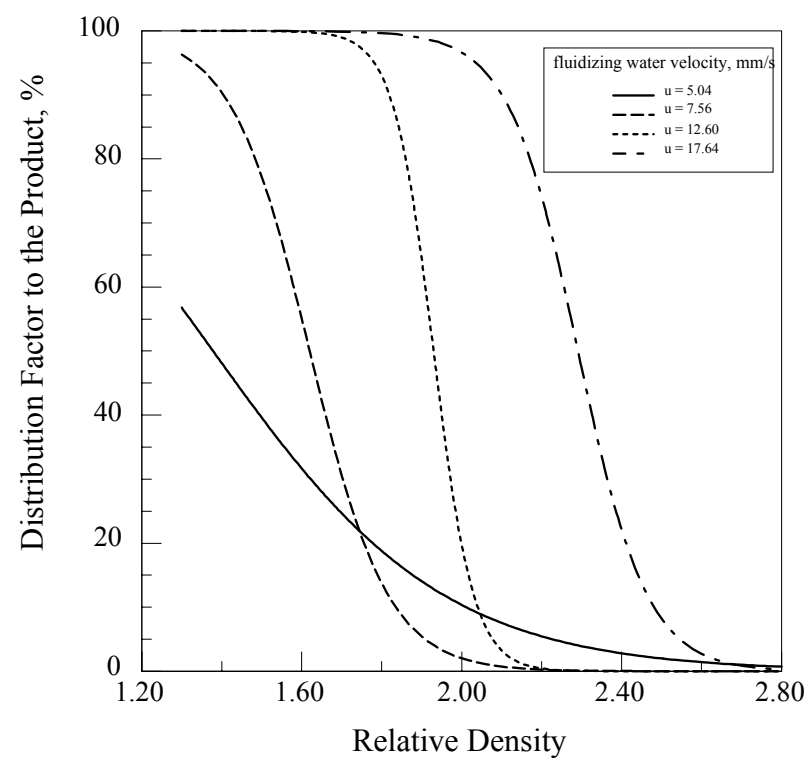

Figure 8.12 Distribution curves at various fluidizing water velocities in a baffled column (particle size: $1 \mathrm{~mm}$ )

The hindered-settling velocity is determined by the suspension density and fluidizing water velocity. In the open column, at low fluidizing water velocity, the drag force is negligible compared to the gravity force. At very low fluidizing flow velocity, SG50 value is low and Ep value is high. The distribution curve is relatively flat. Although the fluidized-bed suspension gradually increases because of particle accumulations, the fluidizing water velocity is insufficient to cause coarse particles to separate and lower lose of coarse clean coal in the underflow. By increasing the fluidizing water velocity, the relative velocity between a particle and liquid flow increases, and the drag force increases drastically. The drag forces acting on low density particle surpasses the gravity effect and carries the low density particles upward to the overflow. The gravity force acting on the high density particle is larger than the upward drag force. The particles with high density can still settle to the underflow without much effect by the liquid flow. 
It is a well-known disadvantage of the HSBS that it often suffers from the misplacement of low-density coarse particles to the high-density underflow. At the low fluidizing water velocity, the low density particle has low probability of reporting to the overflow because of low drag forces. This inherent inefficiency can be partially corrected by increasing the fluidizing water velocity to convey the coarse low density particles to the overflow.

By increasing the fluidizing water velocity, the distribution curve has shifted significantly away from low relative density to high relative density. At high fluidizing water velocity, although the suspension density is decreased because of bed expansion, the low density particles are carried upward and hence have no chance of reporting to the reject stream. However, the particles which are higher than 2.4 in relative density, should report to the reject stream because of inlet fluidizing water velocity. Although a less suspension density can be formed at high fluidizing water velocity, a higher density cut-point than suspension density is still achieved because of the drag force effect. It is noted that the density cut-point is always lower than the suspension density, thus the effect if fluidizing water velocity can not be ignored. At extremely high fluidizing water velocity, the drag force becomes dominant and overcomes the gravity force. All particles tend to be elutriated upward and separation deteriorates in spite of large density difference of the particles.

Unlike a conventional fluidized-bed separator, the presence of the structured plates permits a broad range of the fluidizing water velocity while maintaining lower Ep values. Through comparison of Figure 8.11 and Figure 8.12, it is found that the structured plates can help to generate a lower density cut-point than those values in the open column. More important is the fact that in nearly all cases the structured plates assisted to maintain shaper separation than the open column, which is denoted by lower Ep values. This positive 
contribution of structured plates can be attributed to building a high suspension density gradient and enhancement of density separation by fluid rotation in structured plates.

Table 8.4 Parameters used in simulation of fluidizing water velocity effect at high suspension density

\begin{tabular}{||l|l||}
\hline Particle size & $\begin{array}{l}\text { Two size fractions: }-1.0+0.7 \mathrm{~mm} \\
\text { and }-1.4+1.0 \mathrm{~mm}\end{array}$ \\
\hline Particle density & $1.2-2.8 \mathrm{~g} / \mathrm{cm}^{3}, \mathrm{wt} \%$ evenly distributed \\
\hline Inlet water flow velocity & $12.96-45.5 \mathrm{~m}^{3} / \mathrm{m}^{2} \mathrm{~h}$ \\
\hline Superficial fluidizing water velocity & $3.6-12.6 \mathrm{~mm} / \mathrm{s}$ \\
\hline Dry particle feed loading & $2.65-9.28 \mathrm{t} / \mathrm{m}^{2} \mathrm{~h}$ \\
\hline $\begin{array}{l}\text { Coefficient of restitution-particle } \\
\text { collisions }\end{array}$ & 0.1 \\
\hline Coefficient of restitution-wall collisions & 0.1 \\
\hline Friction coefficient & 0.1 \\
\hline Pulsation addition & No \\
\hline Suspension relative density at set point & $1.2 \mathrm{~g} / \mathrm{cm}^{3}$ \\
\hline Set point height & $0.35 \mathrm{~m}$ \\
\hline Simulation time & $30 \mathrm{~seconds}$ \\
\hline
\end{tabular}

From Figures 8.11 and 8.12 , it is also shown that for particle with a size of $1 \mathrm{~mm}$, the particle has a low recovery in both columns at low fluidizing water velocity. Increasing the fluidizing water velocity can improve the separation; however, the high fluidizing water velocity will elutriate the fine particles. One solution is to increase the set point suspension density.

In the following simulations, the density set-point is increased to 1.2. It is interesting to consider the effect of increasing the fluidizing water velocity on the separation at this suspension density value. The parameters used in simulation are list in Table 8.3 and the predicted separation indexes are list in Tables 8.4 and 8.5. The distribution curves are compared for two size fractions $(-1.0+0.7 \mathrm{~mm}$ and $-1.4+1.0 \mathrm{~mm})$ in the open and baffled columns, as shown in Figures 8.13 to 8.16 . At this high suspension density, compared with Figures 8.11 and 8.12 , the recovery of coarse low density particles is increased at the cost of 
lowering the separation efficiency under high fluidizing water velocity. The separation performance indexes are compared for these two size fractions in Figure 8.17 and Figure 8.18 .

Table 8.5 SG50 and Ep for an open column at different inlet water flow velocities at high suspension density

\begin{tabular}{||c|c|c|c|c|c|c|c|c|c|c||}
\hline $\begin{array}{c}\text { Inlet fluidizing } \\
\text { water }\end{array}$ & \multicolumn{2}{|c|}{$1.8 \mathrm{~mm} / \mathrm{s}$} & \multicolumn{2}{|c|}{$3.6 \mathrm{~mm} / \mathrm{s}$} & \multicolumn{2}{|c|}{$5.4 \mathrm{~mm} / \mathrm{s}$} & \multicolumn{2}{c|}{$7.2 \mathrm{~mm} / \mathrm{s}$} & \multicolumn{2}{c|}{$9.6 \mathrm{~mm} / \mathrm{s}$} \\
\hline & SG50 & Ep & SG50 & Ep & SG50 & Ep & SG50 & Ep & SG50 & Ep \\
\hline $\begin{array}{c}\text { Open column } \\
0.7-1.0 \mathrm{~mm}\end{array}$ & 1.548 & 0.151 & 1.745 & 0.153 & 1.837 & 0.183 & 2.078 & 0.119 & 2.175 & 0.151 \\
\hline $\begin{array}{c}\text { Open column } \\
1.0-1.4 \mathrm{~mm}\end{array}$ & 1.339 & 0.181 & 1.515 & 0.100 & 1.579 & 0.069 & 1.739 & 0.131 & 1.849 & 0.140 \\
\hline \hline
\end{tabular}

Table 8.6 SG50 and Ep for a baffled column at different inlet water flow velocities at high suspension density

\begin{tabular}{|c|c|c|c|c|c|c|c|c|c|c|c|c|}
\hline \multirow[t]{2}{*}{$\begin{array}{l}\text { Inlet fluidizing } \\
\text { water }\end{array}$} & \multicolumn{2}{|c|}{$1.26 \mathrm{~mm} / \mathrm{s}$} & \multicolumn{2}{|c|}{$2.52 \mathrm{~mm} / \mathrm{s}$} & \multicolumn{2}{|c|}{$5.04 \mathrm{~mm} / \mathrm{s}$} & \multicolumn{2}{|c|}{$7.56 \mathrm{~mm} / \mathrm{s}$} & \multicolumn{2}{|c|}{$10.16 \mathrm{~mm} / \mathrm{s}$} & \multicolumn{2}{|c|}{$12.60 \mathrm{~mm} / \mathrm{s}$} \\
\hline & SG50 & Ep & SG50 & Ep & SG50 & Ep & SG50 & Ep & SG50 & Ep & SG50 & Ep \\
\hline $\begin{array}{c}\text { Structured } \\
\text { plates } \\
0.7-1.0 \mathrm{~mm}\end{array}$ & 1.610 & 0.118 & 1.704 & 0.123 & 1.833 & 0.159 & 2.038 & 0.135 & 2.170 & 0.144 & 2.488 & 0.221 \\
\hline $\begin{array}{c}\text { Structured } \\
\text { plates } \\
1.0-1.40 \mathrm{~mm}\end{array}$ & & & 1.447 & 0.09 & 1.612 & 0.089 & 1.699 & 0.113 & 1.832 & 0.134 & 1.983 & 0.089 \\
\hline
\end{tabular}

It was shown that the distribution curve in the baffled column has a higher density cutpoint (SG50) values than that in the open column when fluidizing flow velocity is less than 6 $\mathrm{mm} / \mathrm{s}$. Above this fluidizing flow velocity level, the distribution curve takes a lower SG50 value (Figure 8.17). In nearly all cases, the distribution curves have a lower probable error (Ep) value for the baffled column with structured plates than those in the open column (Figure 8.18). Thus, the structured plate helps to increase the recovery of low density particles at low fluidizing flow velocity and thus reduces the mixing of high density particles at high fluidizing water velocity. This is consistent with conclusions by Gavin et al. (1999) that the HSBS performance is improved by increasing the suspension density. 


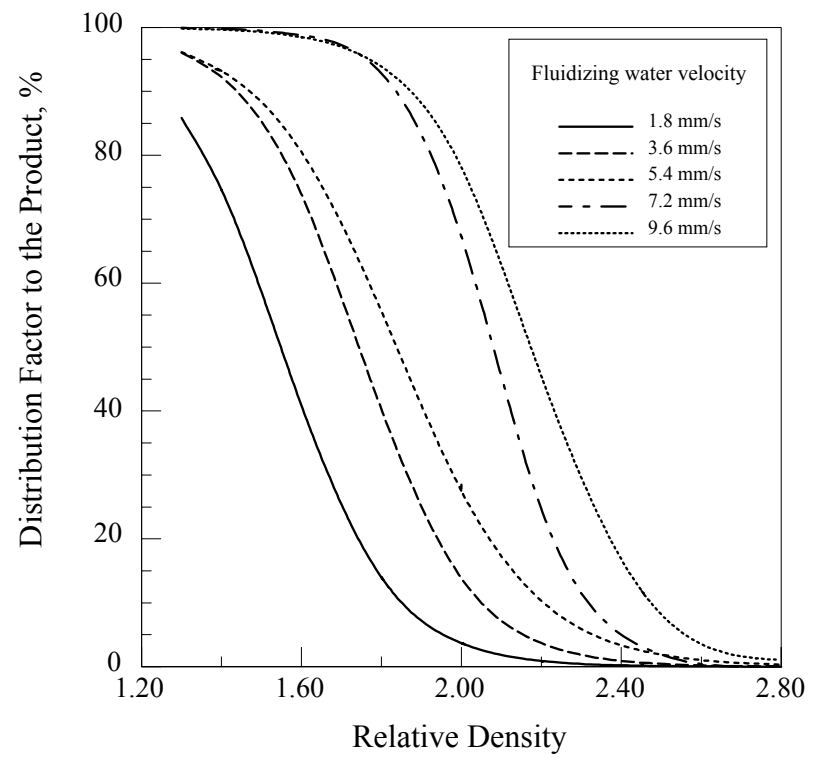

Figure 8.13 Distribution curves for various fluidizing water velocity in an open column (particle size: $-1.0+0.7 \mathrm{~mm}$ )

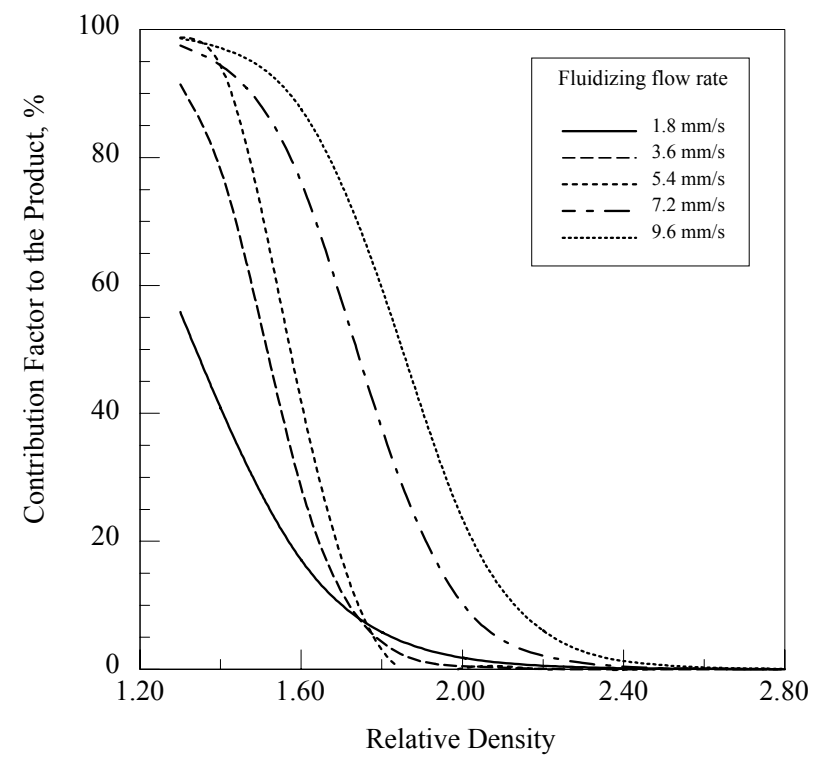

Figure 8.14 Distribution curves for various fluidizing water velocity in an open column (particle size: $-1.4+1.0 \mathrm{~mm}$ ) 


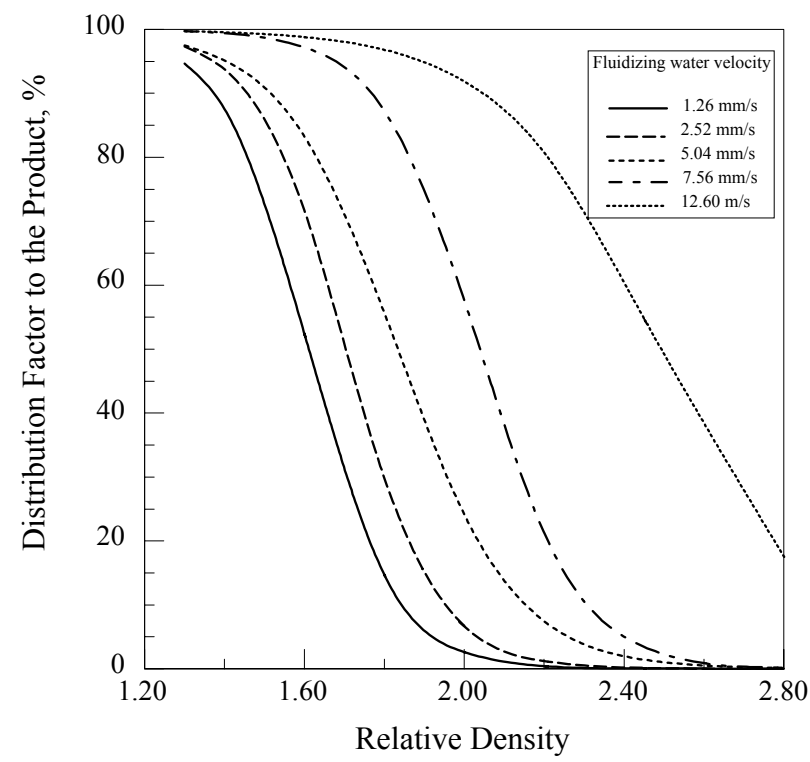

Figure 8.15 Distribution curves for various fluidizing water velocity in a baffled column (particle size: $-1.0+0.7 \mathrm{~mm}$ )

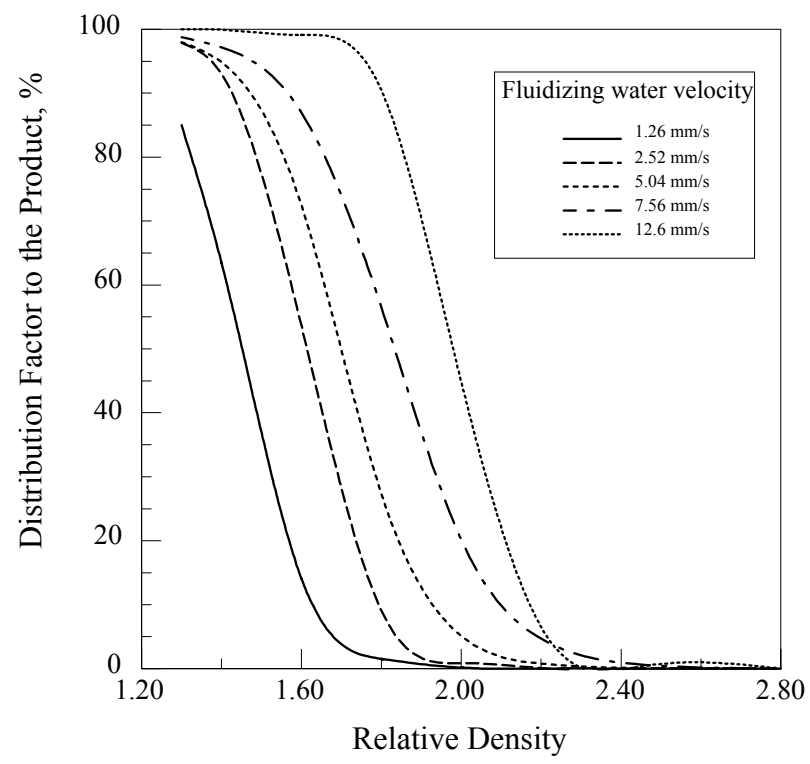

Figure 8.16 Distribution curves for various fluidizing water velocity in a baffled column (particle size: $-1.4+1.0 \mathrm{~mm}$ ) 


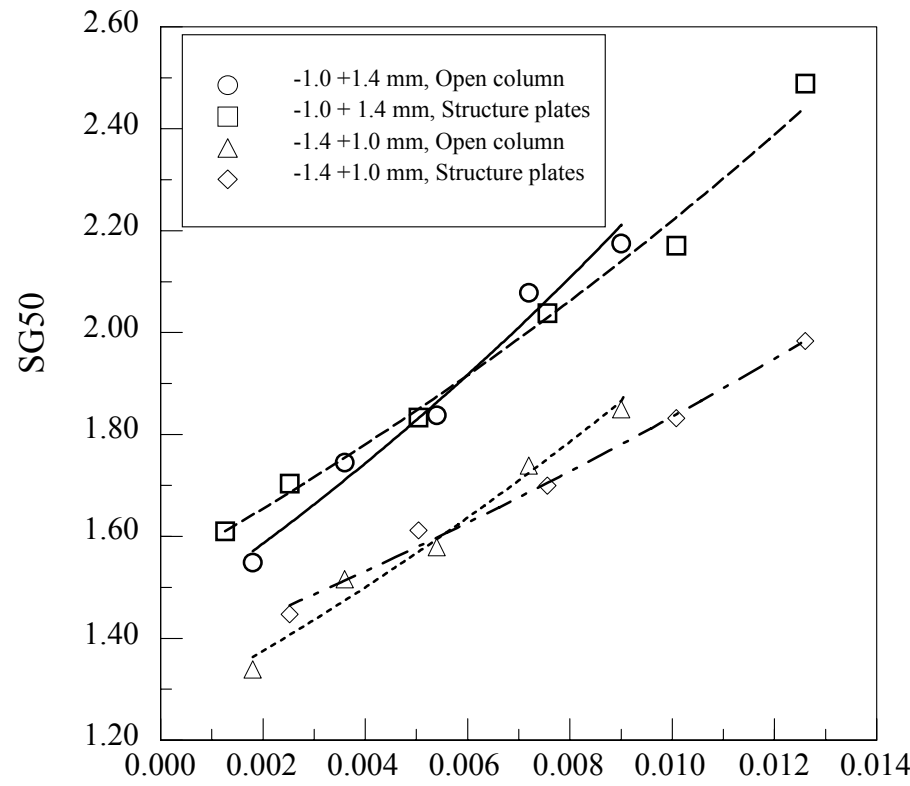

Superficial Fluidizing Water Flow Rate, $\mathrm{m} / \mathrm{s}$

Figure 8.17 Comparisons of the density cut-point as a function of superficial fluidizing water velocity in an open column and a baffled column

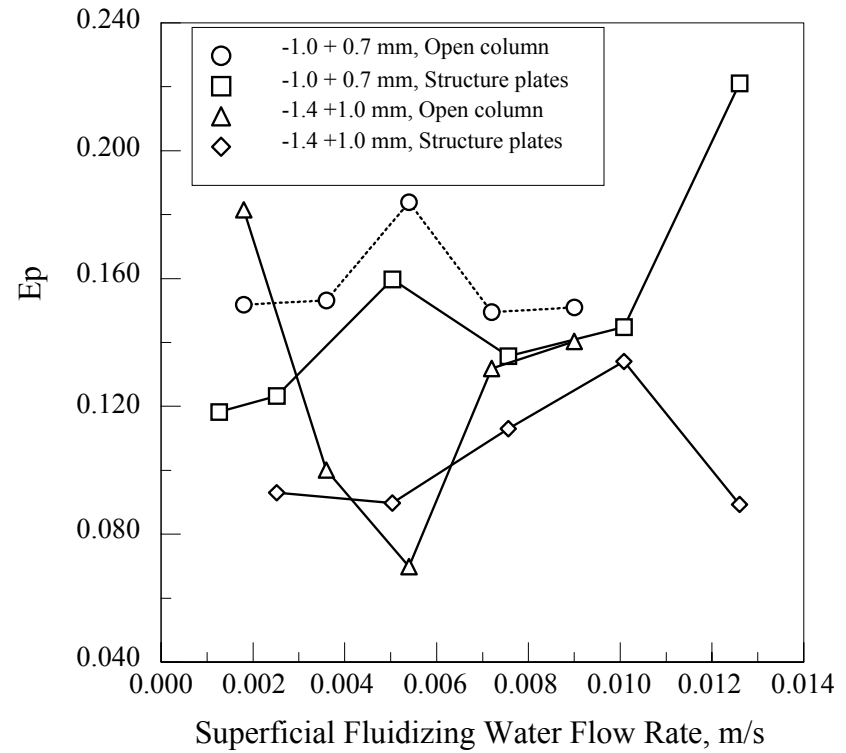

Figure 8.18 Comparisons of the probable error as a function of superficial fluidizing water velocity in an open column and a baffled column 
In a HSBS, an increase in the fluidizing water velocity will generate an immediate and sustained reduction in the suspension density due to the expansion or dispersion of the solid bed. Thus, large lower-density particles become even more likely to report to the reject stream in the open column. However, in the presence of the structured plates, the zigzag inclined plates permit maintenance of the high suspension density near the plate surface at the higher fluidizing water velocity. This will yield a higher recovery of the coarse, lowdensity particles.

The decreased sensitivity of distribution curves to the fluidizing flow velocity in the presence of the structured plates will result in a greater degree of flexibility in its operation, thus overcoming the problem of loss of the relatively coarse, low ash coal particles in HSBS.

Clearly, it is possible to operate a fluidized-bed using fluidizing water velocities that exceeds the terminal settling velocity of a particle. The structured plate helps with the retention of particles within the vessel, overcoming the effects of fluctuations in the fluidizing water velocity. When there are some feed fluctuations, the system could provide a degree of self-control. The extra solid particles can be extracted from the slurry by structured plates and a broad range of suspension concentrations can be sustained at one fluidizing water velocity in response to the feed disturbances.

\subsubsection{Particle Size}

The particle size effect on the density separation performance is obtained for different size fractions in the open and baffled columns are shown in Figures 8.19 and 8.20. Size fractions of $-2+1.4,-1.4+1.0,-1.0+0.7$ and $-0.7+0.25 \mathrm{~mm}$ at a solid concentration of $15 \%$ were used to evaluate the size effect in the density separation. The distribution curves show a trend shifting to higher density with the decrease of particle size. 


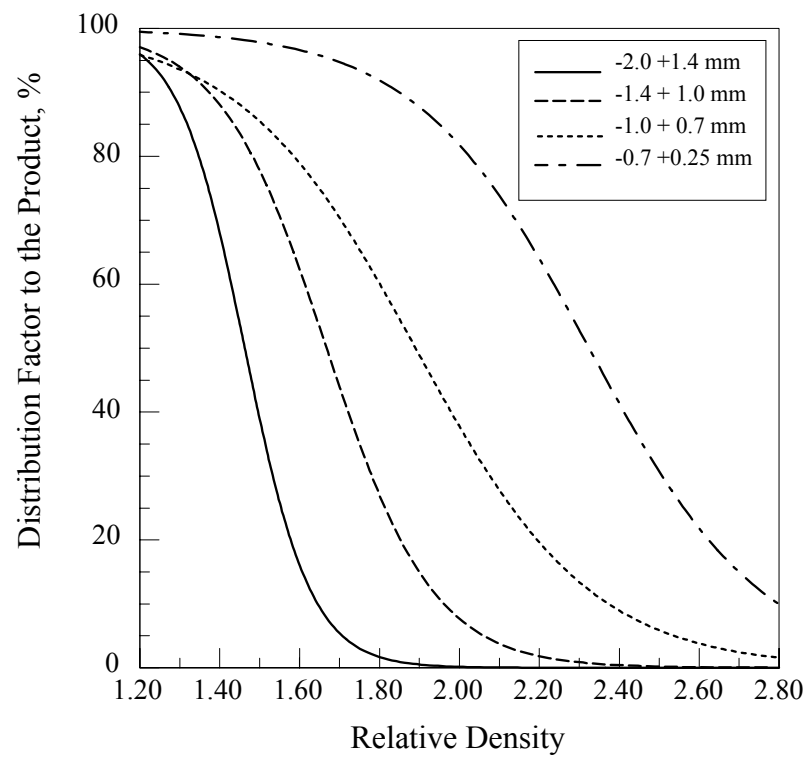

Figure 8.19 Distribution curves for difference size fractions in an open column

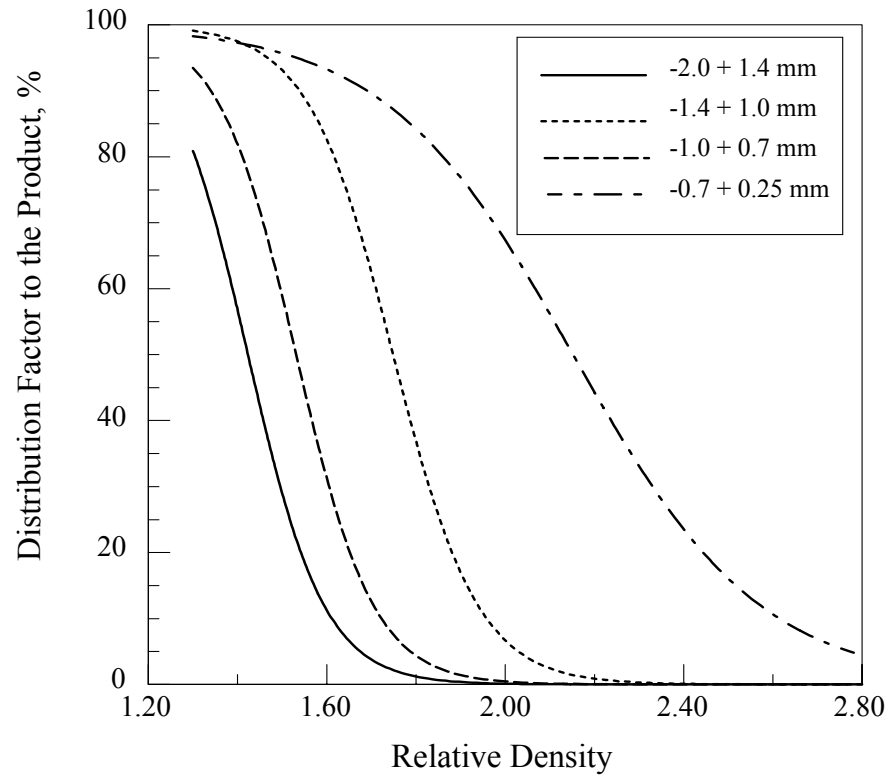

Figure 8.20 Distribution curves for difference size fractions in a baffled column 
Table 8.7 Parameters used in simulation of particle size effect

\begin{tabular}{|l|l|}
\hline Particle size & $-2.0+0.25 \mathrm{~mm}$ \\
\hline Particle density & $1.2-2.8 \mathrm{~g} / \mathrm{cm}^{3}, \mathrm{wt} \%$ evenly distributed \\
\hline Inlet water flow velocity & $19.4 \mathrm{~m}^{3} / \mathrm{m}^{2} \mathrm{~h}$ \\
\hline Superficial fluidizing water velocity & $5.04 \mathrm{~mm} / \mathrm{s}$ \\
\hline Dry particle feed loading & $2.65 \mathrm{t} / \mathrm{m}^{2} \mathrm{~h}$ \\
\hline $\begin{array}{l}\text { Coefficient of restitution-particle } \\
\text { collisions }\end{array}$ & 0.1 \\
\hline $\begin{array}{l}\text { Coefficient of restitution-wall } \\
\text { collisions }\end{array}$ & 0.1 \\
\hline Friction coefficient & 0.1 \\
\hline Pulsation addition & $\mathrm{No}$ \\
\hline $\begin{array}{l}\text { Suspension relative density at set } \\
\text { point }\end{array}$ & $1.2 \mathrm{~g} / \mathrm{cm}^{3}$ \\
\hline Set point height & $0.35 \mathrm{~m}$ \\
\hline Simulation time & $30 \mathrm{~seconds}$ \\
\hline
\end{tabular}

Table 8.8 SG50 and Ep for different size fractions in the open and baffled columns

\begin{tabular}{|c|c|c|c|c|c|c|c|c|}
\hline \multirow{2}{*}{ size } & \multicolumn{2}{|c|}{$-2.0+1.4 \mathrm{~mm}$} & \multicolumn{2}{|c|}{$-1.4+1.0 \mathrm{~mm}$} & \multicolumn{2}{|c|}{$-1.0+0.7 \mathrm{~mm}$} & \multicolumn{2}{l|}{$-0.7+0.25 \mathrm{~mm}$} \\
\hline & SG50 & Ep & SG50 & Ep & SG50 & Ep & SG50 & Ep \\
\hline Open column. & 1.4629 & 0.0911 & 1.6675 & 0.1467 & 1.8901 & 0.2413 & 2.3244 & 0.2373 \\
\hline Baffled column & 1.4234 & 0.0900 & 1.5314 & 0.0955 & 1.7486 & 0.1047 & 2.1522 & 0.2308 \\
\hline
\end{tabular}

The Ep values, which denote the separation efficiency, increase from 0.0911 to 0.2373 as the particle size decreases in the open column, as shown in Figure 8.19. The corresponding density cut-point SG50 increases from 1.4629 to 2.3244 relative density. In the presence of the structured plates, the Ep and SG50 values are all relatively low, and hence the structured plates has a positive effect in density separation, as shown in Figure 8.20. The comparison of Ep and SG50 at the same operating conditions in the open and baffled columns is shown in Figures 8.21 and 8.22 . In the presence of the structured plates, the distribution curve shows lower SG50 and Ep values. At constant SG50 and Ep values, a lower size cut-point can be 
reached in the presence of the structured plates. Thus, the inclusion of structured plates will help to extend its lower separation size limit.

If the solid concentration is increased to $30 \%$, when the particle size is larger than $1 \mathrm{~mm}$, the Ep values change less, as shown in Figures 8.23 and 8.24. The SG50 values in the baffled column are less sensitive to the size effect than that in the open column, and SG50 is higher in the presence of the structured plates with increase of the particle size. Thus, the structure of plates may improve the recovery of low density coarse particles. Overall, in the presence of structured plates, the corresponding distribution curves, at the same operating conditions as those used for open column, have shown the trend of shifting to higher density cut-points while maintaining less SG50 changes and lower Ep values with decreasing sizes. It can be said that the structured plates improve the density separation for fine particle fractions.

\subsubsection{Solid Feed Velocity}

In principle, the fluidization hydraulic loading in the baffled column only needs to be equivalent to that in an open column, given that a superficial fluidizing water velocity is sufficient to just fluidize the bed and in turn maintain a high bed concentration. In the open column, the Ep values tend to increase with the feed velocity, however, the presence of the structured plates can help to achieve a lower Ep. 


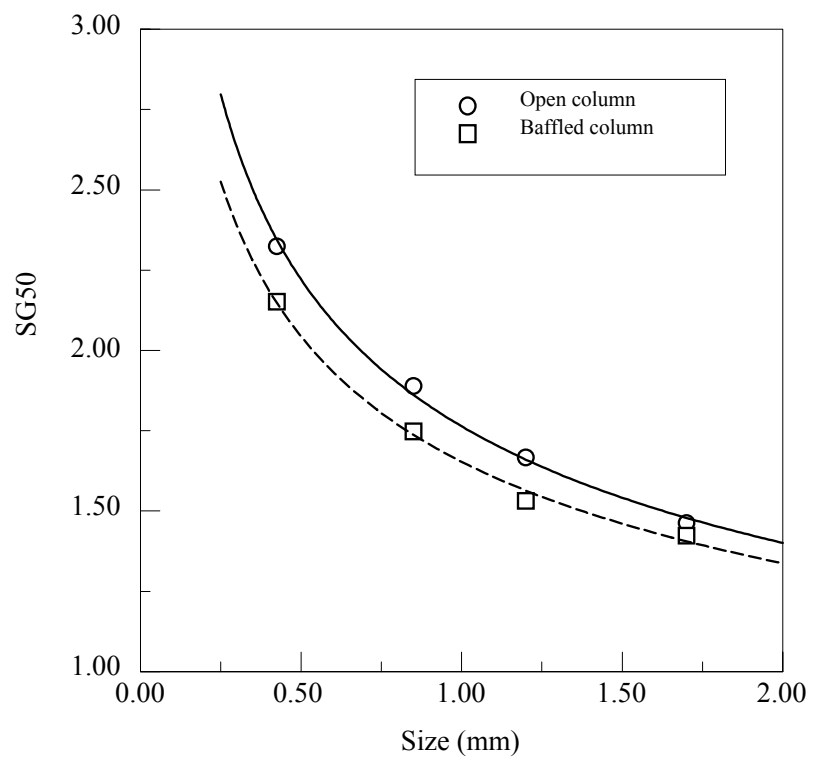

Figure 8.21 Density cut-point values as a function of particle size for an open column and a baffled column (feed solid concentration: 15\%)

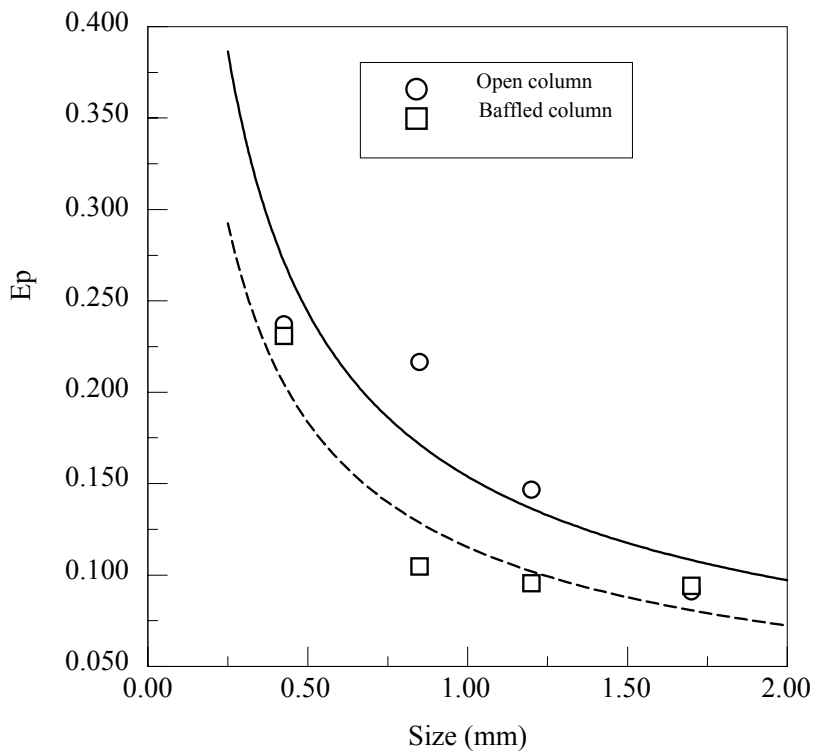

Figure 8.22 Probable error values as a function of particle size for an open column and a baffled column (feed solid concentration: 15\%) 


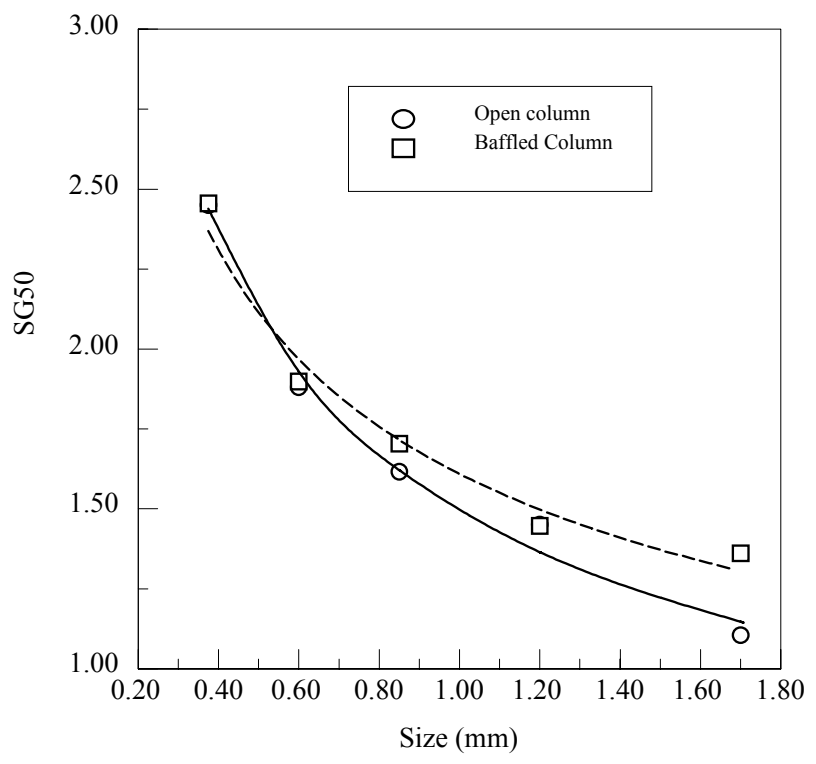

Figure 8.23 Density cut-point values as a function of particle size for an open column and a baffled column (feed solid concentration: $30 \%$ )

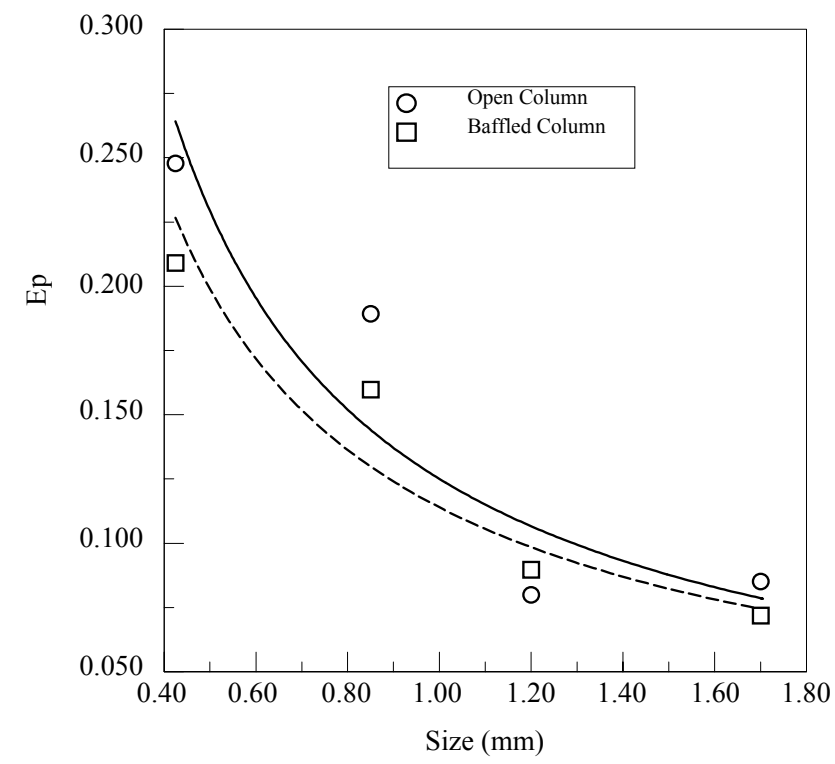

Figure 8.24 Probable error values as a function of particle size for an open column and a baffled column (feed solid concentration: 30\%) 
Table 8.9 Parameters used in simulation of solid feed rate effect at high suspension density

\begin{tabular}{|l|l|}
\hline Particle size & $-1.0+0.7 \mathrm{~mm}$ \\
\hline Particle density & $1.2-2.8 \mathrm{~g} / \mathrm{cm}^{3}, \mathrm{wt} \%$ evenly distributed \\
\hline Inlet water flow velocity & $19.4 \mathrm{~m}^{3} / \mathrm{m}^{2} \mathrm{~h}$ \\
\hline Superficial fluidizing water velocity & $5.04 \mathrm{~mm} / \mathrm{s}$ \\
\hline Dry particle feed loading & $2.65-10.6 \mathrm{t} / \mathrm{m}^{2} \mathrm{~h}$ \\
\hline $\begin{array}{l}\text { Coefficient of restitution-particle } \\
\text { collisions }\end{array}$ & 0.1 \\
\hline $\begin{array}{l}\text { Coefficient of restitution-wall } \\
\text { collisions }\end{array}$ & 0.1 \\
\hline Friction coefficient & 0.1 \\
\hline Pulsation addition & $\mathrm{No}$ \\
\hline $\begin{array}{l}\text { Suspension relative density at set } \\
\text { point }\end{array}$ & $1.2 \mathrm{~g} / \mathrm{cm}^{3}$ \\
\hline Set point height & $0.35 \mathrm{~m}$ \\
\hline Simulation time & $30 \mathrm{~seconds}$ \\
\hline
\end{tabular}

Table 8.10 Variations of SG50 and Ep with solid feed rate in open and baffled columns

\begin{tabular}{|c|l|l|l|l|l|l||}
\hline $\begin{array}{c}\text { Fluidizing water } \\
\text { velocity }\end{array}$ & \multicolumn{2}{|l|}{$2.65 \mathrm{t} / \mathrm{m}^{2} \mathrm{~h}$} & \multicolumn{2}{l|}{$7.95 \mathrm{t} / \mathrm{m}^{2} \mathrm{~h}$} & \multicolumn{2}{l|}{$10.6 \mathrm{t} / \mathrm{m}^{2} \mathrm{~h}$} \\
\hline & SG50 & Ep & SG50 & Ep & SG50 & Ep \\
\hline Open column & 1.8901 & 0.2452 & & & & \\
\hline Baffled column & 1.7486 & 0.1047 & 1.8531 & 0.1689 & 1.9848 & 0.2089 \\
\hline
\end{tabular}

Thus the baffled column can be operated at a higher feed velocity at same required Ep values. The effect of solid feed velocity for size fraction of $-1.0+0.7 \mathrm{~mm}$ is shown in Figure 8.25. At solid feed rate of $7.95 \mathrm{t} / \mathrm{m}^{2} \mathrm{~h}$, the baffled column has a similar separation performance to that of the open column operating at a solid concentration of $2.65 \mathrm{t} / \mathrm{m}^{2} \mathrm{~h}$. It is clear that the baffled column can reach a high throughput than the open column without cost of separation performance. 


\subsubsection{Geometric Parameters of Structured Plates}

The effect of structured plates on the density separation performance can be controlled by changing the geometric parameters of the structured plates. In CFD simulation with a twodimensional model, only the distance between two neighboring plates, D, and fold angle, $\alpha$, are considered. These parameters are described in Figure 8.2. In this Figure $\delta p$ is defined as height of corrugation. The simulation results given below are for $\delta p$ is equal to $16 \mathrm{~cm}$.

\subsubsection{Distance between Structured Plates}

The effect of distance between two neighboring structured plates is described in Figure 8.26. The SG50 value increases when two structured plates become closer to each other. The Ep value decreases with when the distance D decreases. Thus, in a small distance of D, the structured plates have more significant effect on density separation. If the D value is too high, no effect of structure plates can be observed, and the separation performance of baffled column is similar to that of the open column. Here, the column will lose the effect of structured plates. If distance is too small, it will bring a strong interaction of particles with the wall of structured plates. Then the pressure drop along the column will increase drastically. Figure 8.27 shows the pressure drop in baffled column with D. When, D is smaller than $0.12 \mathrm{~m}$, the pressure drop starts to increase rapidly. 


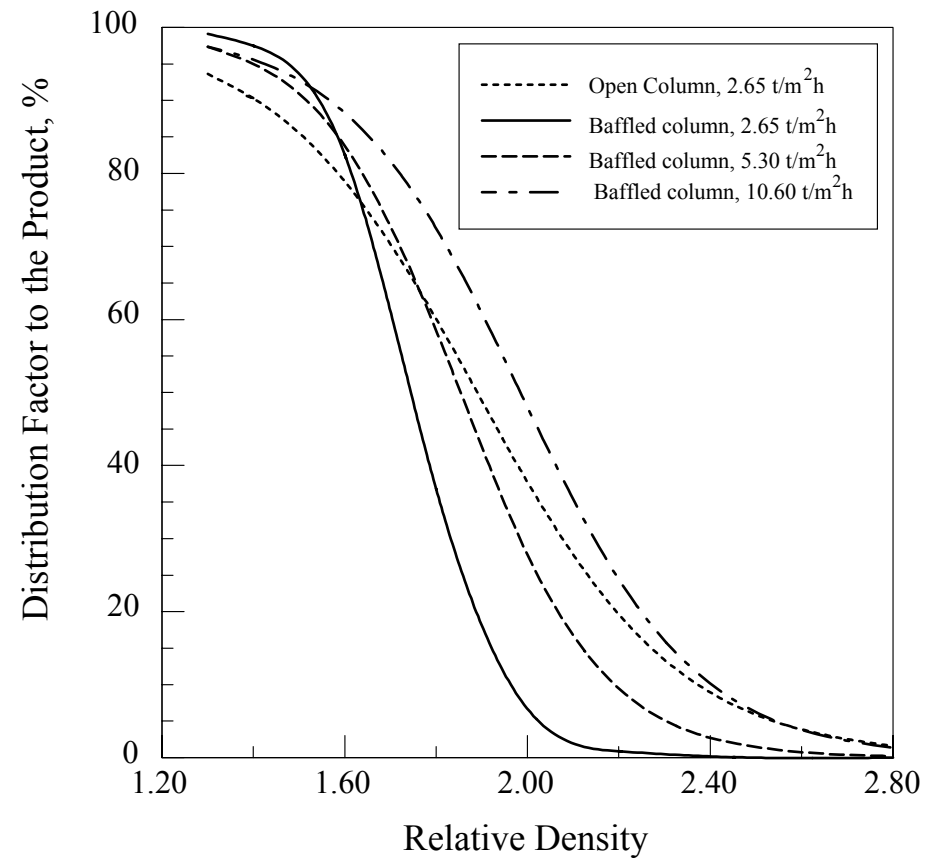

Figure 8.25 Effect of solid feed on separation performance in different columns

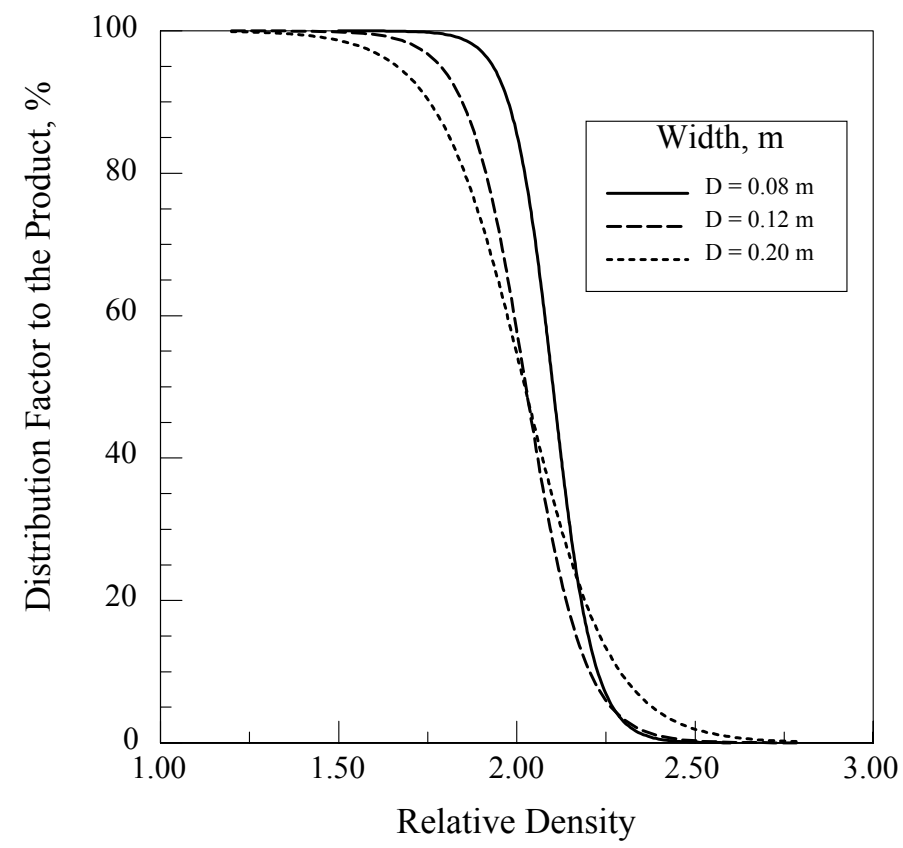

Figure 8.26 Effect of distance of structured plates in a baffled column 


\subsubsection{Fold Angle}

The effect of fold angle on liquid flow in the channel between two neighboring structured plates is described as axial water velocity in Figure 8.28 . The velocity in the middle of the channel decreases with fold angle. As shown in Figure 8.29, the SG50 increases when smaller fold angles are used, while Ep values decrease with the decrease of the fold angle. The use of the structured plates in the column shows stronger effect on the density separation with smaller fold angle. The SG50 also increases with smaller fold angles. Figure 8.30 shows the pressure drop in baffled column as a function of fold angle. When fold angle is below 90 degrees, the pressure drop starts to increase rapidly. As fold angle increases, the plate wall will become steep and more particles will have high probability of settling to the bottom of the column.

To obtain optimum separation performance, the dimensions of structure plates should be carefully designed. Because of the limitation of the 2D model, the effect of structured plates is not fully simulated in this study. In order to maintain the positive effect of structured plates without causing a large pressure drop, the constant fold angle of 90 degrees, corrugation height of $16 \mathrm{~cm}$, and distance between structured plates of $12 \mathrm{~cm}$ are used in structured plate simulations unless mentioned in specific cases.

\subsubsection{Pulsating /Fluidizing Water Effect}

One objective in this study is to improve our understanding the mechanisms involved in the particle stratification and the relative importance of pulsating flow in fine particle separation. This section describes the application of a two-dimensional Euler-Lagrange model to the pulsating process in a baffled HSBS, and presents some results 


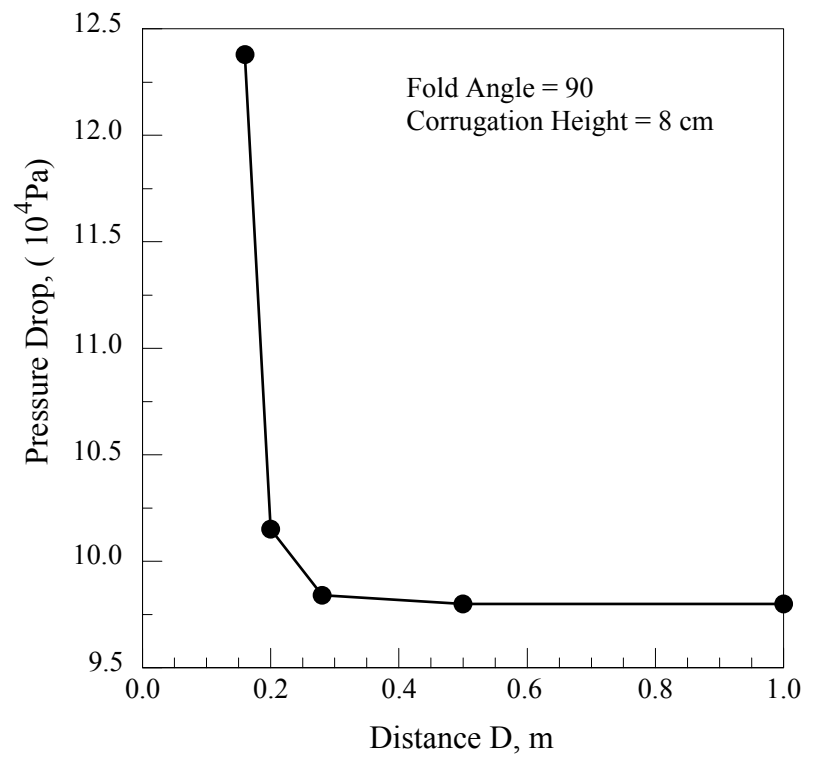

Figure 8.27 Pressure drop along baffled column with distance D
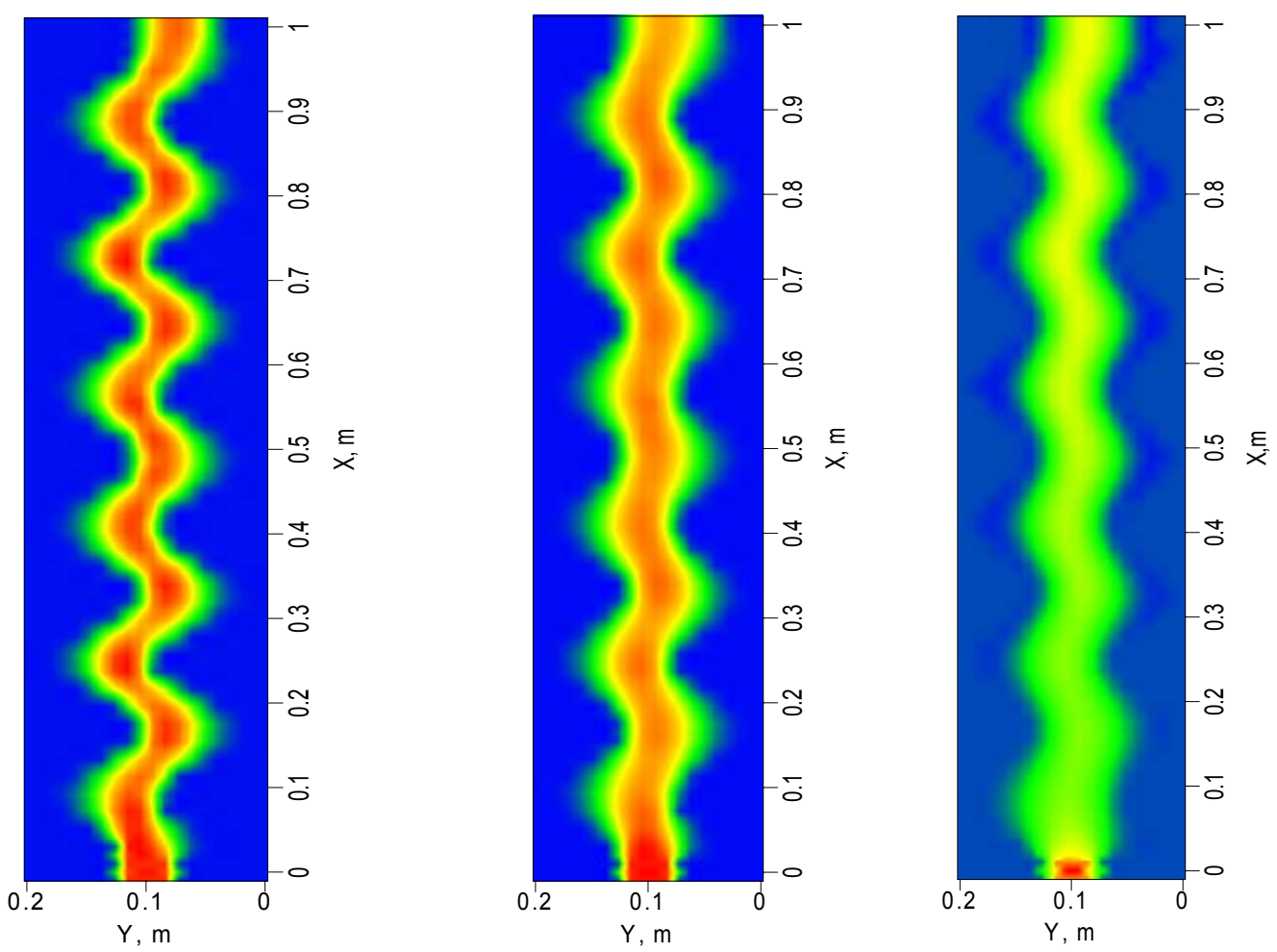

$$
\alpha=80^{\circ}
$$$$
\alpha=90^{\circ}
$$$$
\alpha=100^{\circ}
$$

Figure 8.28 Axial water velocity image map with fold angle 


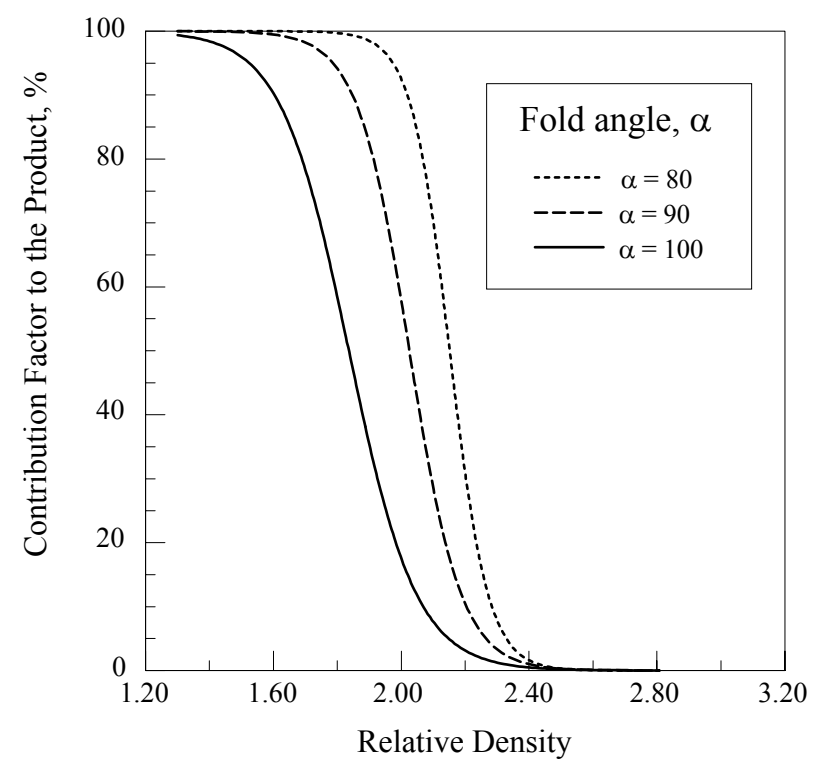

Figure 8.29 Effect of fold angle in a baffled column

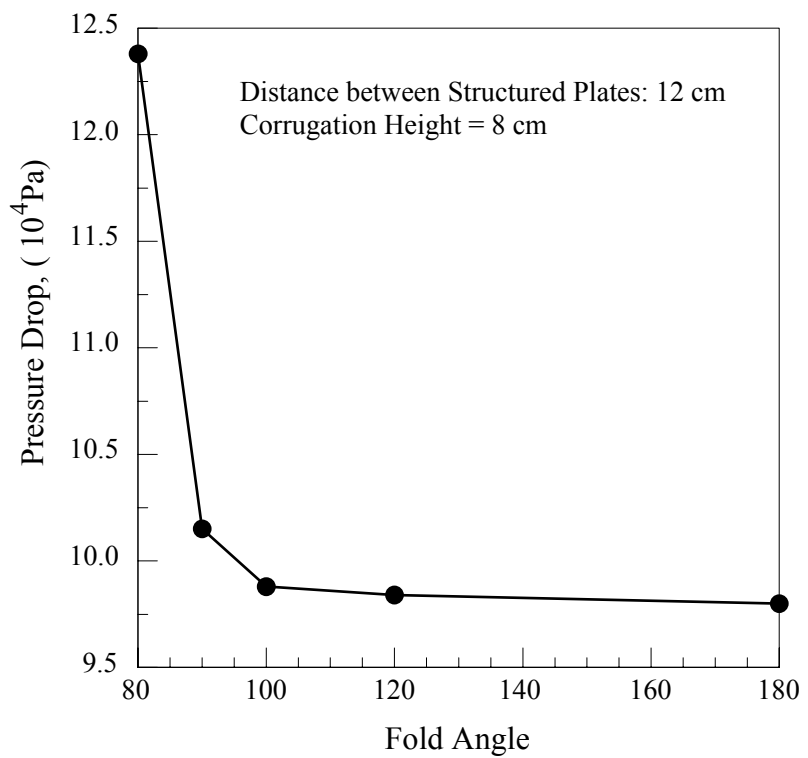

Figure 8.30 Pressure drop along a baffled column with fold angle 
from the simulation of the effect of amplitude and frequency of pulsating water flow on the fine particle separation.

The Euler-Lagrange model can describe the motion of every individual particle, such that the integration of individual particle behavior results in a macroscopic behavior of particle assembly in a pulsating fluid. Various operating parameters affecting the stratification process are investigated. These parameters include amplitude and frequency of pulsation flow and feed characteristics.

The pulsation stoke form is shown in Figure 8.31 and consists of a constant upward stream, on which is supposed by a small, sinusoidal pulsation. There can be stratification of particles by density only if the heavy particles displace the light particles upwards. This displacement, however, is possible only by direct contact or by impact. This means that the loosening of the particle bed brought about by the up-stoke must not to be too great. Since the particles move freely and independent of one another in high speed flows, this leads to the elutriations of all particles.

\subsubsection{Flow Velocity}

Figures 8.32 to 8.34 show the effect of addition of fluidizing water in baffled column on density separation performance. Table 8.10 lists the parameters used for simulation and Table 8.11 shows the SG50 and Ep values with addition of the pulsation in the baffled column at various fluidizing water velocities. It can be seen from Figure 8.33 and 8.34 that the SG50 and Ep values are less sensitive to the changes of fluidizing water velocity. At low fluidizing water velocity, the pulsation has a positive effect on the separation. However, if the fluidizing water velocity is too high, the pulsation begins to show a negative effect (larger value of Ep) on the separation. It might be caused by strong mixing effect by high pulsation 


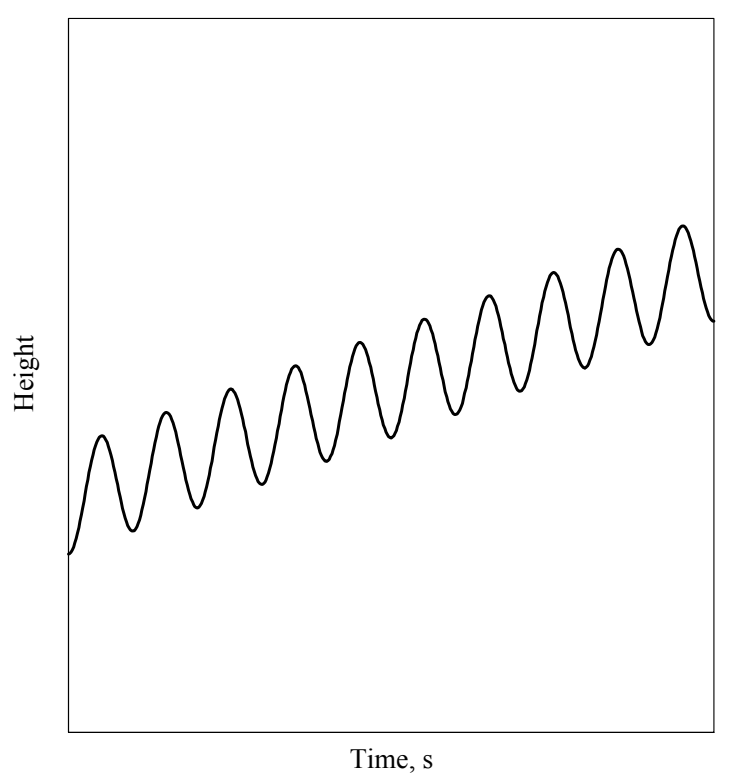

Figure 8.31 Wave form of liquid flow

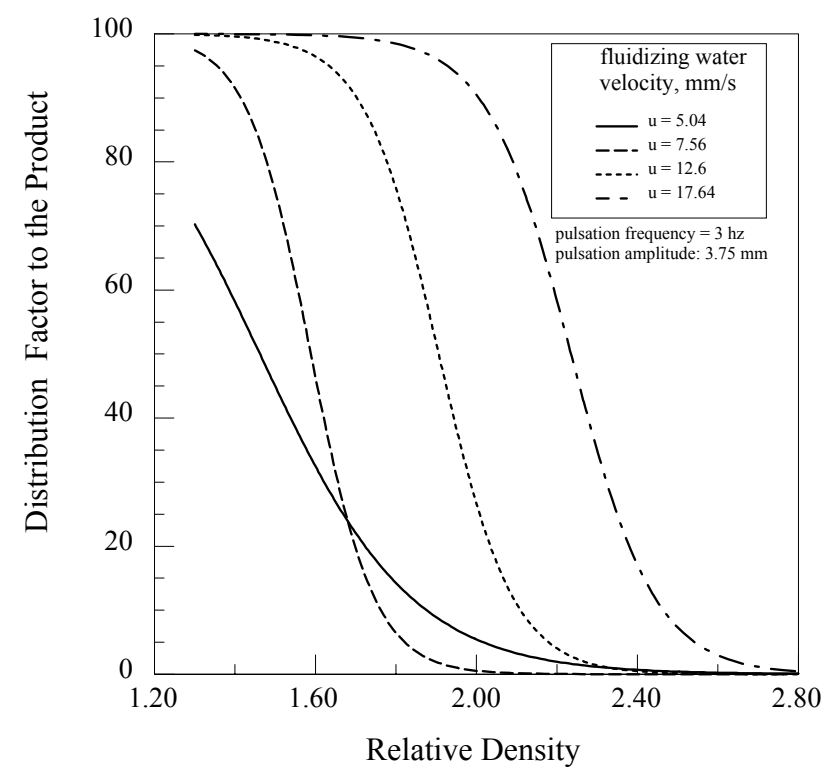

Figure 8.32 Effect of fluidizing water velocity on separation performance in a baffled column with pulsating flow 


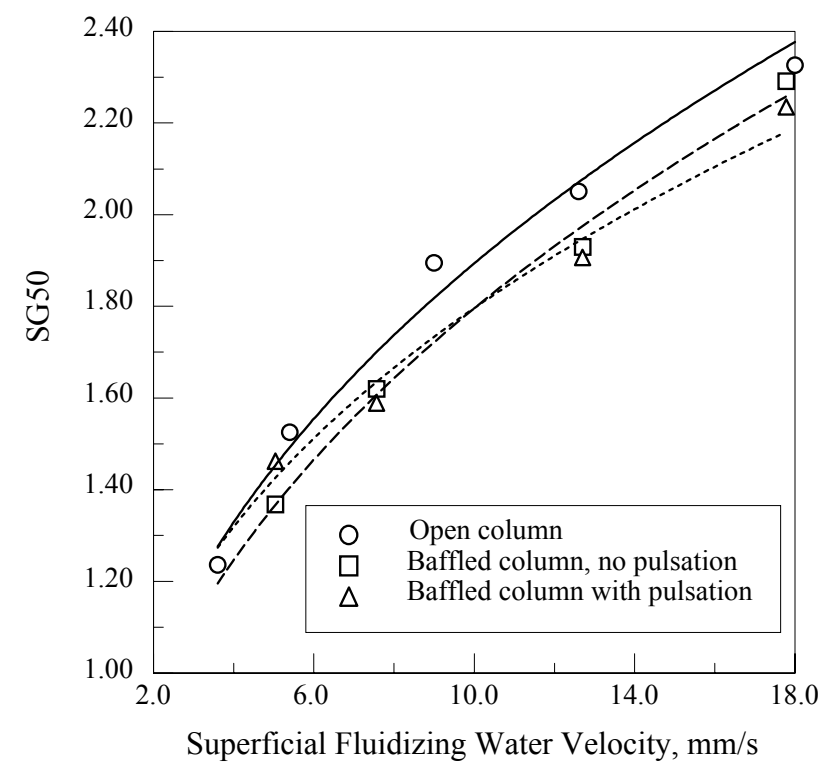

Figure 8.33 Effect of pulsation on SG50 in a baffled column

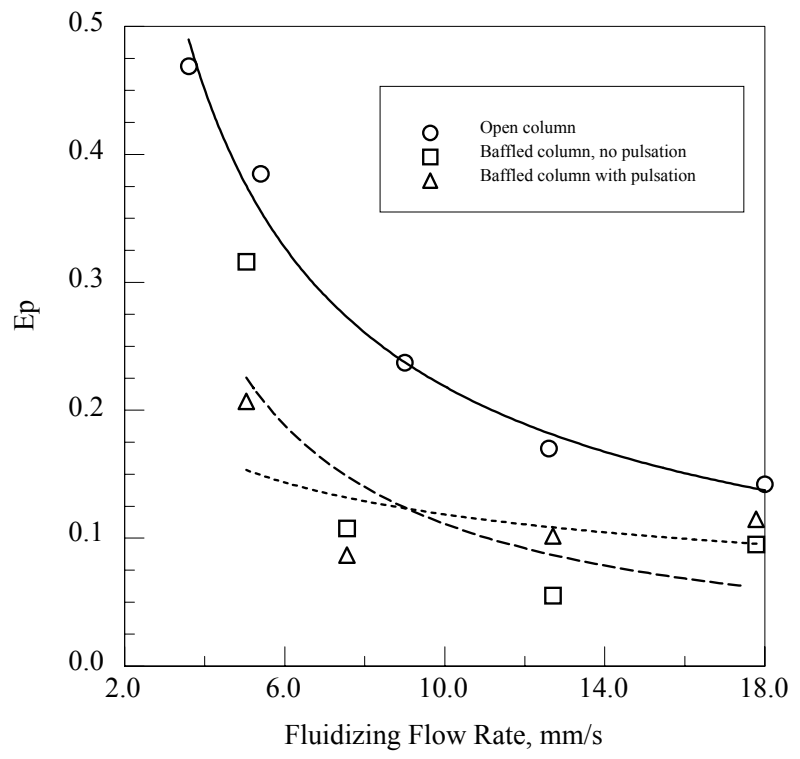

Figure 8.34 Effect of pulsation on Ep in a baffled column 
flow which will destroy the stratification.

Table 8.11 Parameters used in the simulation of fluidizing water velocity effect at low suspension density in the baffled column

\begin{tabular}{|l|l|}
\hline Particle size & $1 \mathrm{~mm}$ \\
\hline Particle density & $1.2-2.8 \mathrm{~g} / \mathrm{cm}^{3}, \mathrm{wt} \%$ evenly distributed \\
\hline Inlet water flow velocity & $12.96-45.5 \mathrm{~m}^{3} / \mathrm{m}^{2} \mathrm{~h}$ \\
\hline Superficial fluidizing water velocity & $3.6-12.6 \mathrm{~mm} / \mathrm{s}$ \\
\hline Dry particle feed loading & $2.65-9.28 \mathrm{t} / \mathrm{m}^{2} \mathrm{~s}$ \\
\hline $\begin{array}{l}\text { Coefficient of restitution-particle } \\
\text { collisions }\end{array}$ & 0.1 \\
\hline $\begin{array}{l}\text { Coefficient of restitution-wall } \\
\text { collisions }\end{array}$ & 0.1 \\
\hline Friction coefficient & 0.1 \\
\hline Pulsation addition & Amplitude $=3.75 \mathrm{~mm}$, frequency $=3 \mathrm{~Hz}$ \\
\hline Suspension relative density set point & $1.15 \mathrm{~g} / \mathrm{cm}^{3}$ \\
\hline Set point height & $0.35 \mathrm{~m}$ \\
\hline Simulation time & $30 \mathrm{~seconds}$ \\
\hline
\end{tabular}

Table 8.12 SG50 and Ep values for baffled column at various feed water velocities

\begin{tabular}{|c|c|c|c|c|c|c|c|c|}
\hline $\begin{array}{c}\text { Inlet pipe water } \\
\text { flow velocity }\end{array}$ & \multicolumn{2}{|c|}{$\mathrm{U}=5.04 \mathrm{~mm} / \mathrm{s}$} & \multicolumn{2}{c|}{$\mathrm{U}=7.56 \mathrm{~mm} / \mathrm{s}$} & \multicolumn{2}{c|}{$\mathrm{U}=12.6 \mathrm{~mm} / \mathrm{s}$} & \multicolumn{2}{l|}{$\mathrm{U}=17.64 \mathrm{~mm} / \mathrm{s}$} \\
\hline $\begin{array}{c}\text { Baffled column } \\
\text { without pulsation } \\
\text { flow }\end{array}$ & $\mathrm{SG50}$ & $\mathrm{Ep}$ & $\mathrm{SG} 50$ & $\mathrm{Ep}$ & $\mathrm{SG} 50$ & $\mathrm{Ep}$ & $\mathrm{SG} 50$ & $\mathrm{Ep}$ \\
\cline { 2 - 10 } & 1.4621 & 0.2068 & 1.5886 & 0.0868 & 1.9063 & 0.1017 & 2.2352 & 0.1146 \\
\hline
\end{tabular}

\subsubsection{Frequency Effect}

The amplitude and frequency of a jigging cycle are the main operating variables in this section. Any changes of frequency of pulsation resulted in variation in the amplitude of the jigging cycle.

The modeling and simulation are carried out for the cases where the amplitude of pulsation was fixed at $3.75 \mathrm{~mm}$, but the frequency of pulsation was varied at four levels: 1,2 , 3, 5 and10 Hz. Table 8.12 lists the parameters used in the simulation and Table 8.13 shows SG50 and Ep values with frequencies of pulsation in the baffled column. Figures 8.35 and 
8.36 present the model predictions of frequency effects. It is clear that increase in the frequency of pulsation increases the degree of stratification. However, if the frequency is too high, Ep values increase drastically. Thus there exists an optimal combination of amplitude and frequency.

Table 8.13 SG50 and Ep for different columns at different inlet water flow velocities

\begin{tabular}{|l|l||}
\hline Particle size & $1 \mathrm{~mm}$ \\
\hline Particle density & $1.2-2.8 \mathrm{~g} / \mathrm{cm}^{3}, \mathrm{wt} \%$ evenly distributed \\
\hline Inlet water flow velocity & $27.2 \mathrm{~m}^{3} / \mathrm{m}^{2} \mathrm{~h}$ \\
\hline $\begin{array}{l}\text { Superficial fluidizing water } \\
\text { velocity }\end{array}$ & $7.6 \mathrm{~mm} / \mathrm{s}$ \\
\hline Dry particle feed loading & $5.3 \mathrm{t} / \mathrm{m}^{2} \mathrm{~s}$ \\
\hline $\begin{array}{l}\text { Coefficient of restitution-particle } \\
\text { collisions }\end{array}$ & 0.1 \\
\hline $\begin{array}{l}\text { Coefficient of restitution-wall } \\
\text { collisions }\end{array}$ & 0.1 \\
\hline Friction coefficient & 0.1 \\
\hline Pulsation addition & Amplitude $=3.75 \mathrm{~mm}$, frequency $=1-10 \mathrm{~Hz}$ \\
\hline $\begin{array}{l}\text { Suspension relative density at set } \\
\text { point }\end{array}$ & $1.15 \mathrm{~g} / \mathrm{cm}^{3}$ \\
\hline Set point height & $0.35 \mathrm{~m}$ \\
\hline Simulation time & $30 \mathrm{~seconds}$ \\
\hline
\end{tabular}

Table 8.14 SG50 and Ep values in baffled column at various pulsating frequencies

\begin{tabular}{||c|c|c|c|c|c|c|c|c||}
\hline $\begin{array}{c}\text { Frequency, } \\
\text { Hz }\end{array}$ & \multicolumn{2}{|c|}{1} & \multicolumn{2}{c|}{3} & \multicolumn{2}{c|}{5} & \multicolumn{2}{c||}{10} \\
\hline $\begin{array}{c}\text { Baffled } \\
\text { column }\end{array}$ & SG50 & Ep & SG50 & Ep & SG50 & Ep & SG50 & Ep \\
\cline { 2 - 9 } & 1.5885 & 0.0864 & 1.5886 & 0.0868 & 1.655 & 0.1386 & 1.7438 & 0.0624 \\
\hline \hline
\end{tabular}




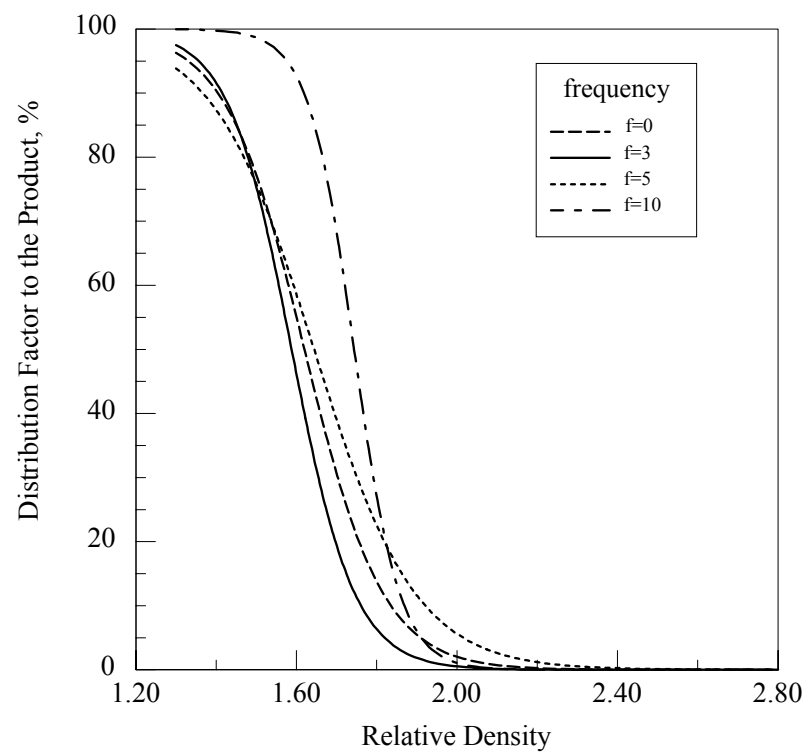

Figure 8.35 Effect of pulsation frequency on separation performance in a baffled column

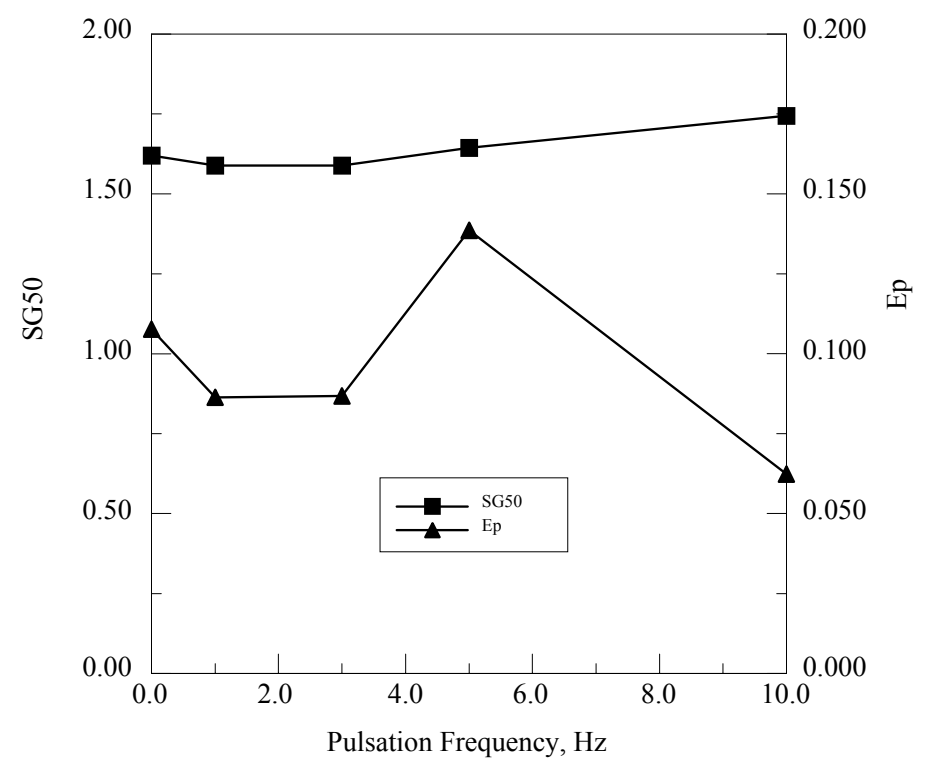

Figure 8.36 Effect of pulsation frequency on SG50 and Ep values in a baffled column 


\subsubsection{Amplitude Effect}

Table 8.14 lists the parameters for the simulation. Table 8.15 shows effect of pulsation amplitudes on the density separation in a baffled column. Figures 8.37 and 8.38 shows the effect of increasing amplitude. From the variations of SG50 and Ep plots, the most efficient stratification was achieved at an amplitude range of 0.8 to $1.2 \mathrm{~cm}$. However, if amplitude is over $1.5 \mathrm{~cm}$, it was found after observing the animation that the peak water velocity exceeded the terminal velocity of the lower density particles. The column was acting as a form of upward current classifier, with the high density particles being progressively moved further away from the bottom.

Table 8.15 Parameters used in simulation of pulsating amplitude effect

\begin{tabular}{|l|l|}
\hline Particle size & $1 \mathrm{~mm}$ \\
\hline Particle density & $1.2-2.8 \mathrm{~g} / \mathrm{cm}^{3}, \mathrm{wt} \%$ evenly distributed \\
\hline Inlet water flow velocity & $27.2 \mathrm{~m}^{3} / \mathrm{m}^{2} \mathrm{~h}$ \\
\hline Superficial fluidizing water velocity & $7.6 \mathrm{~mm} / \mathrm{s}$ \\
\hline Dry particle feed loading & $5.3 \mathrm{t} / \mathrm{m}^{2} \mathrm{~s}$ \\
\hline $\begin{array}{l}\text { Coefficient of restitution-particle } \\
\text { collisions }\end{array}$ & 0.1 \\
\hline $\begin{array}{l}\text { Coefficient of restitution-wall } \\
\text { collisions }\end{array}$ & 0.1 \\
\hline Friction coefficient & 0.1 \\
\hline Pulsation addition & $\mathrm{Amplitude}=3.75-15 \mathrm{~mm}$, frequency $=3$ \\
$\mathrm{~Hz}$
\end{tabular}

Table 8.16 SG50 and Ep values for baffled column at various pulsation amplitudes

\begin{tabular}{||c|c|c|c|c|c|c|c|c||}
\hline Amplitude, $\mathrm{mm}$ & \multicolumn{2}{|c|}{3.75} & \multicolumn{2}{c|}{7.5} & \multicolumn{2}{c|}{11.25} & \multicolumn{2}{|c|}{15} \\
\hline Baffled column & SG50 & Ep & SG50 & Ep & SG50 & Ep & SG50 & Ep \\
\cline { 2 - 9 } & 1.5886 & 0.0864 & 1.5932 & 0.0764 & 1.5858 & 0.0818 & 1.6857 & 0.1091 \\
\hline
\end{tabular}




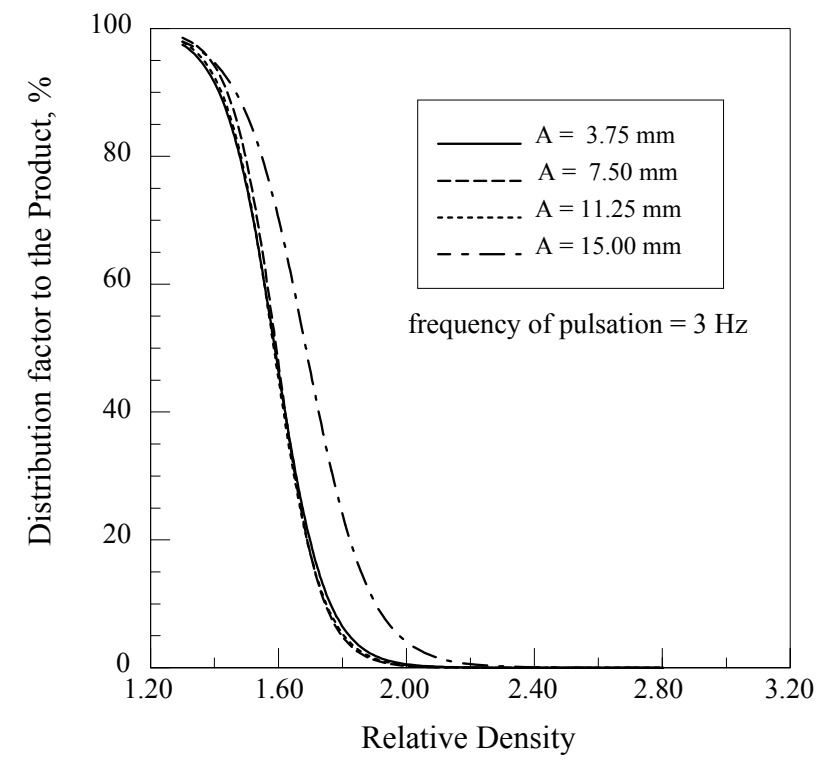

Figure 8.37 Variations of distribution curves with amplitude in a baffled Column

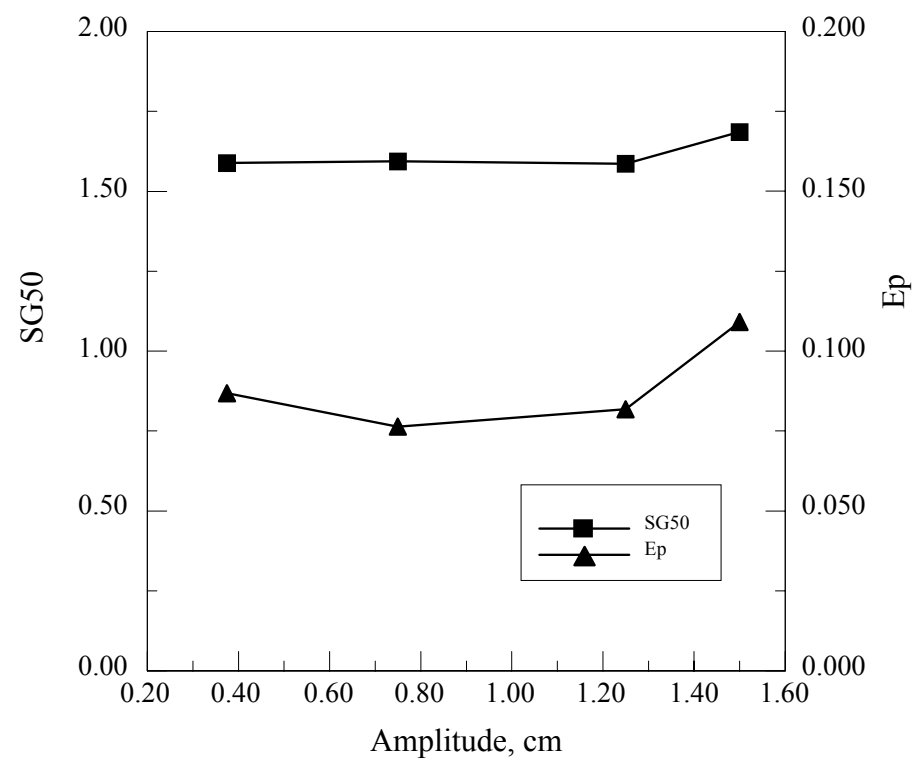

Figure 8.38 Effect of pulsation amplitude on SG50 and Ep values in a baffled column 
There exists critical combination ranges for frequency and amplitude of pulsation. Beyond the ranges, the separation results deteriorated and the separation might be even worse than the open column. At the beginning, the increase of the frequency or amplitude causes the particles between structured plates begin to suspend and disperse. The misplaced particle can have a chance to get separated again. As the pulsating frequency or amplitude increases, the stroke intensifies, even the largest light particles as well as the number of intermediate sized heavy particles go to the top of the column, thus result in a high density cut-point.

\subsection{Summary}

- The coarse, low density particles in the hindered-settling bed separator are easily lost in the underflow, except at low fluidizing water velocity where a higher suspension density is generated. However, a low fluidizing water velocity limits the throughput of the separator.

- Through laboratory performance tests, it was shown that the implementation of structured plates in an open column can improve density separation performance by drastically increasing the throughput.

- The addition of structured plates can decrease the sensitivity of density cut-points to the fluctuations of feed rate, and get a shaper density separation.

- Simulation results show that, the easy formation of a relatively high suspension density gradient and liquid rotating in bended areas of structured plates contribute to the enhancement of separation performance. The distance between plates and the fold angle of structured plates used in the column will also affect the density separation performance. A small fold angle and closer arrangement of structured plates will 
generate a high density cut-point with lower probable errors but might generate a higher pressure drop along the columns.

- The addition of a pulsation flow in the baffled column can increase density separation performance with the optimal combination of frequency and amplitude of pulsating flow. 


\section{Chapter 9 Conclusions}

With the fast growth of computer techniques and advances in Computational Fluid Dynamics (CFD), the direct simulation of multiple phase phenomena in a solid-liquid fluidized-bed becomes feasible. To ensure the quality of the Euler-Lagrange/Baldwin-Lomax method, an efficient, accurate and robust results form of CFD is essential. Solution methodologies for the time-dependent, two-dimensional, incompressible Navier-Stokes equations have been verified by applying them to two cases. One is a hindered-settling bed separator and another case is the baffled column with structured plates. By comparing the simulation results with laboratory experimental and in plant test data, it was shown that the use of CFD methodology has favorable accuracy and efficiency.

Numerical simulation of multiple phase flows in HSBS, a solid-liquid fluidized-bed system, is still a big task due to the requirement for handling an excessively large number of particles with different densities and sizes individually to resolve the particle separation. In the course of this study, a robust approach for reconstructing the behavior of the particles in a liquid field, namely the Euler-Lagrange/Baldwin-Lomax approach, has been applied and validated. This approach can be used to compute the liquid phase flow field and also to describe the movement of the particles and further make a prediction of particle separation results. By using this technique, the liquid flow pattern in an HSBS can be produced with good accuracy. Thus this makes it possible to obtain a better understanding of fluid flow pattern in the solid-liquid system. This detailed information is not available from existing models for HSBS such as population balance model and solid concentration convention- 
diffusion model. In the Euler-Lagrange/Baldwin-Lomax model, it is also possible to track the movement of each particle. The detailed properties of particles can be defined and used to model and track the trajectory of each particle. The integration of individual particle behavior results in a description of the macroscopic behavior of particle assembly in solid-liquid fluidized-bed.

A finite volume discretization and iterations, based on the SIMPLE (Semi-Implicit Pressure Linked Equations) algorithm over staggered grids have been used for the numerical solution. The drag force, pressure gradient force, added mass force, Mangus force and Saffman force have been considered into the computation of the particle movement. The liquid and solid phase coupling is made through updating of the liquid volume fraction and the source force term that accounts for the momentum transfer from the particles to the liquid.

To start multiple phase flow simulation of the HSBS, the behavior of the isolated particle in different flow conditions is simulated. The hindered-settling velocities for different particles are compared to check the size and density effect. The suspension density and viscosity are also changed to determine the response of the particles to the properties changes of liquid phase. The natural packing of the particles with different sizes is simulated to check the particle-particle and wall-particle collision models. The stratification of uniformly sized particles with different densities in a pulsating flow was also computed. The model is also used to successfully simulate the inversion phenomena in a solid-liquid fluidized-bed. In this phenomenon, a binary suspension of relatively fine, heavy particles and coarse, light particles are separated and mixed with increasing of the fluidizing water velocity. Finally, these two groups of particles switch the position with coarse, light particle 
on the bottom and heavy, fine particles on the top of bed. Many slip velocity models failed to predict this inversion phenomena. The Euler-Lagrange/Baldwin-Lomax model was then used to solve the multiple phase flow. A good agreement between predictions and measurements was obtained. The influences of particle properties, suspension properties and different operating parameters have been studied.

The second test was mainly to determine the influence of the addition of the structured plates into the HSBS. The results show that the presence of the structured plates significantly affects the density separation of the fine particles. Three parameters have been simulated including fluidizing water velocity, particle size, and solid feed velocity. With addition of the structured plates, the baffled column showed a decreased sensitivity to the fluidizing flow velocity changes, shaper separation, lower separation size limit, and higher throughput than the open column.

In the third case, the frequency and amplitude of pulsation flow were investigated. Although only part of the particle size fractions were used, this simulation has important benefits for calculation of particle trajectories in vortices of structured plates. It also revealed that there exists an optimal frequency and amplitude, which is particle size dependent. 


\section{RECOMMENDATIONS}

Accuracy and efficiency are the primary issues in the simulation of multiphase flow using CFD tools. In this study, the model is second order accurate for liquid phase flow and fourth order in integration of movement equation of the particles. To achieve a better accuracy, a higher order discretization schemes will need to be investigated.

To perform the simulation of three dimensional case multiphase flows, sufficient fine

grid resolution and a larger number of the particles have to be used to capture characteristics of density separation. A single processor run will hardly provide enough capability to meet this demand. Thus, parallel computations with domain decomposition techniques are desirable.

The addition of bubbles in the HSBS for mineral separation is available in the mineral industry. The simulation of three phase flows in HSBS is not possible until details of mechanisms of attachment and detachment of bubble to particles are available for modeling. The suitable models which describe the surface properties of particles are also needed. However to include these phenomena in the Euler-Lagrange simulation is rather difficult and should be considered or undertaken as a long-term future work.

Finally for density particle separation in HSBS, the effects of discrete particle/turbulence interaction should be eventually included. When using one equation model (Bald-Lomax), it is possible to account for some of these effects through explicit modeling of dispersion rate of sub-grid turbulent kinetic energy. 


\section{BIBLIOGRAPHY}

Al-Naafa, M. A., and Selim, M. S., 1992, "Sedimentation of monodisperse and bidisperse hard-sphere colloidal suspension”, A. I. Ch. E. Journal, Vol. 38. No. 10, pp. 1618-1630.

Armstong, D. G., 1963, "Variation in Stroke Waveform in a Laboratory Jig", Trans. Inst. Min. Metall., Vol. 73, pp. 643-662.

Asif, M., 1997, "Modeling of multi-solid liquid fluidized-beds", Chemical Engineering Technology, Vol. 20, pp. 485-490.

Baldwin, B.S., and Lomax, H., 1978, "Thin layer approximation and algebraic model for separated turbulent flows", AIAA Paper, N 257.

Barnea, E. and Mizrahi, J., 1973, "A generalized approach to the fluid dynamics of particulate systems. Part I”, Chemical Engineering Journal, Vol. 5, pp. 171-189.

Beck, A. J. G. and Holtham, P. N., 1993, "Computer simulation of particle stratification in a two-dimensional batch jig", Mineral Engineering, Vol. 6, pp. 523-536.

Bethell, P.J., 1988, "Current and future processing flow sheets", Industrial Practices of Fine Coal Processes, Chapter 30, pp. 317-329.

Brauer, H., and Theile, H., 1973, Bewegung von partikelschwarmen, Chem. Ing. Tech. Vol. 45, No. 13, pp. 909-912.

Clift, R., Grace, J.R. \& Weber, M.E., 1978, “Bubbles, Drops and Particles”, Academic Press, New York, pp. 100-121.

Crowe, C.T., Sommerfeld, M. \& Tsuji, Y., 1998 "Fundamentals of gas-particle and gasdroplet flows", CRC Press, Boca Raton, USA, pp. 50-98.

Concha, F. and Almendra, E.R., 1979, "Settling velocities of particulate systems - 2. Settling of suspensions of spherical particles", International Journal of Minerals Processing, Vol. 6, pp. 31-41.

Dai, Q., 1999, "Simulation of packed column jigging”, Thesis, West Virginia University, Morgantown, West Virginia

Dandy, D. S. and Dwyer, H. A., 1990, "A sphere in shear flow at finite Reynolds number: Effect of shear on particle lift, drag, and heat transfer", J. Fluid mech., Vol. 216, pp. 381-410. 
Davies, R., 1968, "The experimental study of the differential settling of particles in suspension at high concentration", Powder Technology, Vol. 2, pp. 43-51.

Davis, R. H., and Gecol, H., 1994, "Hindered-settling function with no empirical parameters for polydisperse suspension”, A. I. Ch. E. Journal, Vol. 40. No.3, pp. 570-575.

Dennis, S. C. R., Singh, S. N. \& Ingham, D. B., 1980, “The steady flow due to the rotating sphere at low and moderate Reynolds number", J. Fluid Mechanics, Vol. 101, pp. 257-279.

Delnoji, E., Lammers, F.A., Kuipers, J.A.M. and van Swaaij, W. P. M., 1997, "Dynamic simulation of dispersed gas-liquid two phase-flow using a discrete bubble model", Chemical Engineering Science, Vol. 52, No. 9, pp. 1429-1458.

Enwald, H. perano, E. and Almstedt, A. E. 1996, "Eulerian two-phase flow theory applied to fluidization”, Int. Multiphase Flow, Suppl. 22, pp. 21-66.

Galvin, K. P., Pratten, S. J. and Nicol, S. K., 1999, "Dense medium separation using a teetered bed separator", Mineral Engineering, Vol. 12, No. 9, pp. 1059-1081.

Galvin, K. P., Prattern, S. J., and Nguyen-Tran-Lam, G., 1999, “A generalized empirical description for particle slip velocities in liquid fluidized-beds", Chemical Engineering Science, Vol. 54, pp. 1045-1052.

Galvin, K. P., Doroodchi, E., Callen, A. M., Lambert, N. and Prattern, S. J., 2002, "Pilot plant trial of the reflux classifier", Mineral Engineering, Vol. 15, pp. 19-25.

Galvin, K. P, Nguyentranlam, G., 2002, "Influence of parallel inclined plates in a liquid fluidized-bed system”, Chemical Engineering Science, Vol. 57, pp. 1231-1234.

Garside, J., and Al-Dibouni, M. R., 1979, "Particle mixing and classification in liquid fluidized-beds", Trans. IchemE., Vol. 57, pp. 95-103.

Honaker, R. Q. and Mondal, K., 2000, "Dynamic modeling of fine coal separation in a hindered-bed classifier”, Coal Preparation, Vol. 21, pp. 211-232.

Jinnouchi, Y. and Kawashima, S., 1979, "How to predict and optimize the pulsation in the air-pulsated jig", $8^{\text {th }}$ International Coal preparation Congress., pp.157-167.

Jinnouchi, Y., Kita, S., Tanaka, M., Sawada, Y., 1984, "New trends in theory and technology of the air pulsated jigs in Japan”, Miner Metall. Process., Vol. 1, pp.76-82.

Kim, B. H., Klima, M. S., and Cho, H., 2003, "Modeling of hindered-settling column separations", Advance in Gravity Concentration, edt. by R. Q. Honaker and W. R. Forrest, pp. 39-63. 
Klima, M. S. and Luckie, P. T., 1988, "Evaluating coal preparation processes", Coal Preparation, Vol. 6, pp. 91-101.

Kohmuench, J. N., 2000, "Improving efficiencies in water-based separators using mathematical analysis tools", Dissertation, Virginia Polytechnic Institute and State University, Blacksburgh, Virginia

Kojovic, T., and Whiten, W. J., 1993, "Modeling and simulation of cone classifiers", XVIII International Mineral Processing Congress, Sydney, pp. 251-256.

Launder, B. E. and Spalding, D. B., 1974, "The numerical computation of turbulent flows", Computational methods in Applied Mechanics and Engineering, Vol. 3, pp. 269-289.

Littler, A., 1986, "Automatic hindered-settling classifier for hydraulic sizing and mineral beneficiation”, Transactions, Institute of Mining and Metallurgy, Vol. 95, pp. 133-138.

Lockett, M. J., and Al-Habboody, H. M., 1973, "Differential settling by size of two particle species in a liquid", Trans. Instn. Chem. Engrs., Vol. 51, pp. 281-292.

Low, G.S. and Bhattacharya, S.N., 1984, "On the Study of Maximum Solids Concentration in Suspension Rheology", The Twelfth Australian Chemical Engineering Conference, Melbourne, Australia, August 26-29, Paper 23b, pp. 805-812.

Mackie, R. I., Tucker, P., and Wells, A., 1987, "Mathematical model of the stokes hydrosizer", Transactions, Institution of Minerals and Metallurgy, Section C: Mineral Processing and Extractive Metallurgy, Vol. 96, September, pp. 130-136.

Mankosa, M.J. and Carver, R.M., 1995, "Processing of chopped wire waste material using the Floatex Density Separator", Third International Symposium on Recycling of Metals and Engineered Materials, edt. by P.B Queneau and R.D. Peterson, The Minerals, Metals, and Materials Society, pp. 111-120.

Mankosa, M.J., Stanley, F.L. and Honaker, R.Q., 1995, "Combining hydraulic classification and spiral concentration for improved efficiency in fine coal recovery circuits", High Efficiency Coal Preparation, ed. S. K. Kawatra, SME, Littleton, Colorado, pp. 99-107.

Masliyah, J.H., 1979, "Hindered-settling in a Multi-Species Particle System”, Chemical Engineering Science, Vol. 34, pp. 1166-1168.

Mayer, F. W., 1964, "Fundamentals of a Potential Theory of the Jigging Process", Proc. of $7^{\text {th }}$ Int. Miner. Proc. Cong., Gordon and Breach, New York, Part 2, pp. 75-86.

Mei, R., 1992, “An approximation expression for the shear lift force on a spherical particle at finite Reynolds number”, Int. J. Multiphase Flow, Vol. 18, pp. 145-147. 
Mirza, S., and Richardson, J. F., 1979, "Sedimentation of suspension of particles of two or more sizes", Chemical Engineering Science, Vol. 15, pp. 230-242.

Mishra, B. K., Mehrotra, S. P., 1998, "Modeling of particle stratification in jigs by the discrete element method", Mineral Engineering, Vol. 11, No. 6, pp. 511-522.

Mondal, K., 1997, "A dynamic population balance model for a hindered bed classifier for fine particle separations”, Thesis, Southern Illinois University, Carbondale, Illinois.

Moritomi, H., Iwase, T., and Chiba, T., 1982, "A comprehensive interpretation of solid layer inversion in liquid fluidized-beds", Chemical Engineering Science, Vol. 37, No. 12, pp. 1751-1757.

Odar, F. and Hamilton, W.S., 1964, "Forces on a sphere accelerating in a viscous fluid", J. Fluid Mech., Vol. 18, pp. 302-314.

Oesterlé, B. \& Petitjean, A., 1993, "Experiments on the lift of a spinning sphere in a range of intermediate Reynolds number", Experiments in Fluids, Vol. 25, pp. 16-22.

Oesterlé, B. \& Bui Dinh, T., 1998, "Simulation of particle-to-particle interactions in gas-solid flows”, Int. J. Multiphase Flow, Vol. 19, pp. 199-211.

Patankar, S. V. and Spalding, D. B., 1972, "A calculation procedure for heat, mass and momentum transfer in three-dimensional parabolic flows", Int. J. Heat, Mass Transfer, Vol. pp. 1787-1797.

Patankar1, S.V., 1980, Numerical Heat Transfer and Fluid Flow, Hemisphere Publishing Co., New York, pp. 113-135.

Peng, F. F., Dai, Q., and Yang, D. C., 2002, "Analysis of packed column jig for fine coal separation", Coal Preparation, Vol. 22, No. 4, pp. 199-207.

Peng, F. F. and Xia, Y. K., 2004a, "Fluid dynamic modeling of fine particle separation in hindered-settling bed separator by CFD", Preprint 04-33, February, 2004 Annual SME Meeting and Exhibit, Denver, CO., pp. 1-11.

Peng, F. F. Xia, Y. K., and Gu, Z. X., 2004b, "Separation Performance of Hindered-Settling Bed Separators in Fine Coal Cleaning Circuit “, Preprint 04-27, February, 2004 Annual SME Meeting and Exhibit, Denver, CO., pp. 23-25.

Reed, S., Roger, R., Honaker, R. Q. and Mankosa, M. J., 1995, "In-plant testing of the Floatex density separator for fine coal cleaning", Proceedings, $12^{\text {th }}$ International Coal Preparation Conference, Lexington, Kentucky, pp. 163-174.

Reeks, M.W. \& McKee, S., 1984, "The dispersive effect of Basset history forces on particle motion in turbulent flow", Phys. of Fluids, Vol. 27, pp.1573. 
Richardson, J. and Zaki, W., 1954, "Sedimentation and Fluidization: Part I", Transactions of the Institute of Chemical Engineering, Vol. 39, pp. 35-53.

Rizk, M.A. and Elghobashi, S. E., 1989, "A two equation turbulence model for dispersed dilute confined two-phase flow”, Int. J. Multiphase Flow, Vol. 15, pp. 119-133.

Rubinow, S.I. and Keller, J. B., 1961, "The transverse force on spinning sphere moving in a viscous fluid", J. Fluid Mech., Vol.11, pp. 447-459.

Saffman, P.G., 1965, “The lift on a small sphere in a slow shear flow”, J. Fluid Mech., Vol. 22 , pp. 385-400.

Schlichting, H., 1965, Grenzschicht-Theorie. Verlag G. Braun, Karlsruhe

Schiller, L. \& Naumann, A., 1933, "Über die grundlegende Berechnung bei der chwerkraftaufbereitung”, Ver. Deut. Ing. 44, pp.318-320.

Schulze, H.J., 1984, "Physio-Chemical Elementary Processes in Flotation," Developments in Mineral Processing, Vol. 4, Chpt. 5, Elsevier, New York, pp. 238-253.

Simonin, O., Deutsch, E. and Minier, J. P., 1993, "Eulerian predication of the fluid-particle correlated motion in turbulent flows”, Appl. Sci. Research, Vol. 51, pp. 275-283.

Smith, T. N., 1991, "Elutriation of solids from a binary mixture", Chemical Engineering Research and Design, Vol. 15, pp. 398-492.

Sommerfield, M., 1995, "The importance of inter-particle collisions in horizontal gas-solid channel flows", Gas-Particle Flows, edt. by Stokck, et al., ASME, FED-Vol. 228, pp. 335345.

Sommerfield, M., and Huber, N., 1999, "Experimental analysis and modeling of particle-wall collisions”, Int. J. Multiphase Flow, Vol. 25, pp. 1457-1489.

Srinivasan, R., Mishra, B. K., and Mehrotra, S. P., 1999, "Simulation of particle stratification in jigs", Coal Preparation, Vol. 20, pp.50-70.

Swanson, V.F., 1999, "Settling equation for minerals process application", Minerals and Metallurgical Processing, Vol. 16, No. 3, pp. 8-13.

Tavares, L. M. and R. P. King, 1995, “A useful model for the calculation of the performance of batch and continuous jigs", Coal Preparation, Vol. 15, pp. 99-128.

Tavares, L. M., 1999, "Monte Carlo simulations on the potential energy theory of jigging”, Coal Preparation, Vol. 20, pp. 71-83. 
Yang, D. C. and Bozzato, P., 2002, "Multicell jigging and flotation for fine coal cleaning", Proceedings, XIV International Coal Preparation Congress and Exhibition, South African Inst. Mining and Metall., Johannesburgh, pp. 389-393.

Zhang, X. and Davis, R. H., 1990, "Particle classification using inclined settlers in series with underflow recycle", Industrial Engineering and Chemical Research, Vol. 29, No. 9, pp. 1894-1900.

Zimmels, Y., 1983, "Hindered motion of particulate system in Newtonian fluids. acceleration and steady regimes", Powder Technology, Vol. 34, pp. 191-202.

Zimmels, Y., 1990, "Hydrodynamic diffusion of particulates in sedimentation systems", Powder Technology, Vol. 61, pp. 131-141.

Zigrang, D. J., and Sylvester, N. D., 1981, "An explicit equation for particle settling velocities in solid-liquid systems", A. I. Ch. E. Journal, Vol. 27, No. 6, pp. 1043-1044. 


\section{VITA}

Yunkai Xia was born on September 10th, 1966 in Haian County, Jiangsu, the People's Republic of China. Mr. Xia received his secondary education at Xichang Middle School and High School from 1980-1984 in Haian County, Jangsu. He received his Bachelor of Science Degree in Mineral Engineering from China University of Mining \& Technology (CUMT) in July 1988. In April 1991, he received a Master of Science Degree in Mineral Engineering from Beijing Graduate School from CUMT. In May 1991, Mr. Xia was appointed as a teaching assistant, and later became a lecturer at the Department of Mineral Engineering of CUMT. From July 1993 to December 1995, he was an Engineer involved in the Research and Development of Silicon Carbide Whisker Project in Beijing, China.

From January 1995 to June 1999, Mr. Xia was a project manager in Technology Development Department of American Golden State Group Corp., Beijing Division. He involved in some engineering projects in mineral industries such as natural flake purification and development of new frothers for fine coal flotation.

On July 1, 1999, Mr. Xia enrolled in the Master of Science program at the Department of Mining Engineering, West Virginia University, Morgantown, West Virginia. He completed his Master degree in December of 2000 and immediately enrolled in the Ph.D program.

$\mathrm{He}$ is currently a doctoral candidate and a student member of Society of Mining, Metallurgy and Exploration (SME). Mr. Xia married with Zimin Ren in 1992, Beijing, China. They have a nine-year-old son, Suyu. 